Florida International University FIU Digital Commons

$7-8-2010$

\title{
An Investigation of the Relationship Between Antemortem and Postmortem Drug Concentrations in Blood
}

Samantha S. Tolliver

Florida International University, thats.samantha@gmail.com

DOI: $10.25148 /$ etd.FI10120802

Follow this and additional works at: https://digitalcommons.fiu.edu/etd

\section{Recommended Citation}

Tolliver, Samantha S., "An Investigation of the Relationship Between Antemortem and Postmortem Drug Concentrations in Blood" (2010). FIU Electronic Theses and Dissertations. 321.

https://digitalcommons.fiu.edu/etd/321 


\section{FLORIDA INTERNATIONAL UNIVERSITY}

Miami, Florida

\section{AN INVESTIGATION OF THE RELATIONSHIP BETWEEN ANTEMORTEM AND POSTMORTEM DRUG CONCENTRATIONS IN BLOOD}

A dissertation submitted in partial fulfillment of the

requirements for the degree of

DOCTOR OF PHILOSOPHY

in

CHEMISTRY

by

Samantha Shirelle Tolliver

2010 
To: Dean Kenneth Furton

College of Arts and Sciences

This dissertation, written by Samantha Shirelle Tolliver, and entitled An Investigation of the Relationship between Antemortem and Postmortem Drug Concentrations in Blood, having been approved in respect to style and intellectual content, is referred to you for judgment.

We have read this dissertation and recommend that it be approved.

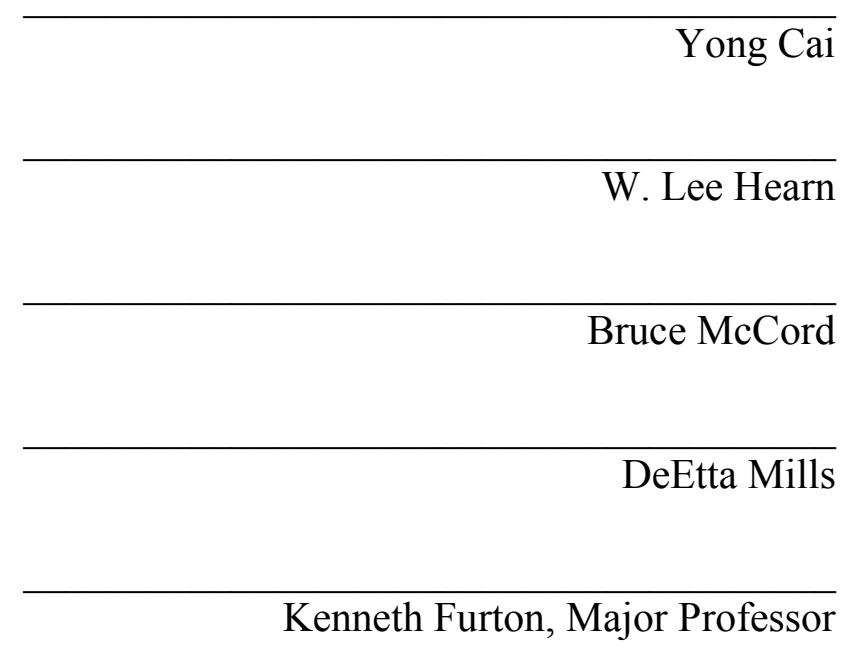

Date of Defense: July 8, 2010

The dissertation of Samantha Shirelle Tolliver is approved.

\begin{tabular}{r}
$\begin{array}{r}\text { Dean Kenneth Furton } \\
\text { College of Arts and Sciences }\end{array}$ \\
\hline Interim Dean Kevin O'Shea \\
University Graduate School
\end{tabular}

Florida International University, 2010 
(C) Copyright 2010 by Samantha Shirelle Tolliver

All rights reserved. 


\section{DEDICATION}

This manuscript is dedicated to the memory of my Great-Grandparents Eddie and Helen Pinkney. Two incredible people who had the foresight to know that with God, a sense of family and education, anything was possible. 


\section{ACKNOWLEDGMENTS}

I would first like to thank my parents, King and Vickie, for being my biggest fans. Thank you for your unconditional love and support. Mom thanks for always being the loudest one in the crowd and allowing me to carve my own path even when you did not agree. Dad thanks for always listening and guiding me without actually telling me what to do. To my brother Edward, thank you for keeping me grounded and reminding me that no one will be there for you like family. Also thank you Grandma Annie for encouraging me to persevere and reminding me that there is nothing wrong with taking time to pamper yourself! Thank you to my great-aunts, aunts, uncles and cousins for all your prayers, words of encouragement and laughs along the way.

I would also like to thank my committee members Dr. Yong Cai, Dr. DeEtta Mills and Dr. Bruce McCord for their constructive criticism and for pushing me to ask and answer questions that at times I thought were beyond my capacity. I could not have asked for a more supportive and thought provoking group of people to help guide me through this journey. A special thanks to my major professor Dr. Kenneth G. Furton, who saw me through my Master's and now my Ph.D.. The years that I have spent under your tutelage have taught me that the mistakes are just as important as the victories. Thank you for teaching me to dream big and for showing me that hard work and a good stiff drink can make all the difference. An additional special thank you goes to my committee member, boss and mentor Dr. W. Lee Hearn. I cannot even begin to express how grateful I am that you saw something in me that I had not yet seen in myself. Thank you for being my sounding board and a safe place to leave all of my insecurities. I hope that I have lived up to your expectations. 
I would also like to thank the Miami-Dade County Medical Examiner Department. You will never know how special it has been for me to know that I have an entire building full of people rooting for me. Thank you to the investigators who have answered all of my questions about the death investigative process, the doctors who very patiently allowed me to interrupt their work flow to answer my many questions and the computer services team for keeping my computer and usb drives virus free. Also thank you to the Chief and Deputy Chief Medical Examiner's, Dr. Bruce Hyma and Dr. Emma Lew, for permitting me to work and research in such a fantastically supportive environment. Last, but certainly not least, thank you to the Assistant Chief Toxicologist George 'Buzz' Hime, and the rest of my colleagues in the Toxicology laboratory who have been dealing with my 6:00 am start time for what must seem like an eternity to them. Buzz, I will forever be grateful for the way you have challenged me and allowed me room to grow. To my supervisors Joe, Julio, Wilmo and Diane thank you for correcting me and teaching me the importance in having pride and integrity in everything that I do. Thanks to my fellow worker bees Roger, Liz, Theresa and Brandi for not allowing me to take myself too seriously and for reminding me that at the end of the day, we are all in this together. Also thanks to Angel for listening even when I know you had something better to do!

So many people have been there to encourage and support me through this process; I sincerely hope that I have not left anyone out. They say it takes a village to raise a child. I am here to tell you that it took a village to get me through this Ph.D.. I could have never done this alone and I am indebted to all who picked me up when I was down. Thank you all. 


\title{
ABSTRACT OF THE DISSERTATION \\ AN INVESTIGATION OF THE RELATIONSHIP BETWEEN ANTEMORTEM AND \\ POSTMORTEM DRUG CONCENTRATIONS IN BLOOD
}

\author{
by \\ Samantha Shirelle Tolliver \\ Florida International University, 2010 \\ Miami, Florida \\ Professor Kenneth Furton, Major Professor
}

In the field of postmortem toxicology, principles from pharmacology and toxicology are combined in order to determine if exogenous substances contributed to ones death. In order to make this determination postmortem and (whenever available) antemortem blood samples may be analyzed. This project focused on evaluating the relationship between postmortem and antemortem blood drug levels, in order to better define an interpretive framework for postmortem toxicology. To do this, it was imperative to evaluate the differences in antemortem and postmortem drug concentrations, determine the role microbial activity and evaluate drug stability.

Microbial studies determined that the bacteria Escherichia coli and Pseudomonas aeruginosa could use the carbon structures of drugs as a source of food. This would suggest prior to sample collection, microbial activity could potentially affect drug levels. This process however would stop before toxicologic evaluation, as at autopsy blood samples are stored in tubes containing the antimicrobial agent sodium fluoride. Analysis of preserved blood determined that under the current storage conditions sodium fluoride 
effectively inhibited microbial growth. Nonetheless, in many instances inconsistent drug concentrations were identified.

When comparing antemortem to postmortem results, diphenhydramine, morphine, codeine and methadone, all showed significantly increased postmortem drug levels. In many instances, increased postmortem concentrations correlated with extended postmortem intervals. Other drugs, such as alprazolam, were likely to have concentration discrepancies when short antemortem to death intervals were coupled with extended postmortem intervals. While still others, such as midazolam followed the expected pattern of metabolism and elimination, which often resulted in decreased postmortem concentrations. The importance of drug stability was displayed when reviewing the clonazepam/ 7-aminoclonazepam data, as the parent drug commonly converted to its metabolite even when stored in the presence of a preservative. In instances of decreasing postmortem drug concentrations the effect of refrigerated storage could not be ruled out. A stability experiment, which contained codeine, produced data that indicated concentrations could continue to decline under the current storage conditions. The cumulative data gathered for this experiment was used to identify concentration trends, which subsequently aided in the development of interpretive considerations for the specific analytes examined in the study. 


\section{TABLE OF CONTENTS}

1.0 Introductory Concepts of Postmortem Toxicology...................................................

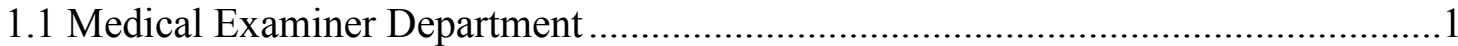

1.2 Medical Examiner's Toxicology Laboratory ............................................... 1

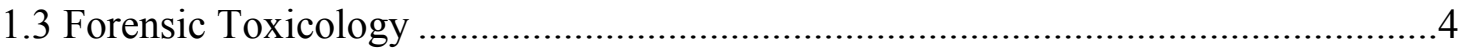

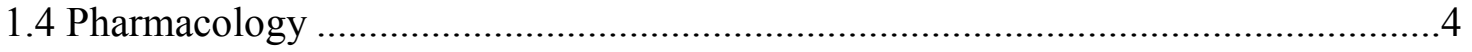

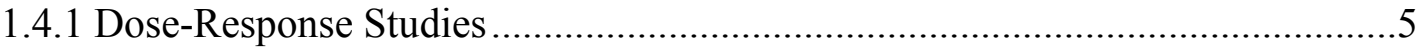

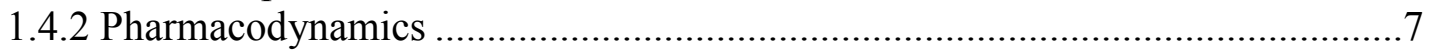

1.4.3 Pharmacokinetics .................................................................................

1.5 Postmortem Processes.................................................................................... 12

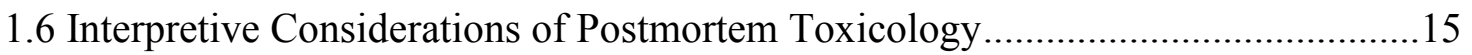

1.6.1 Specimen Choice (Whole blood) and Postmortem Phenomena ......................16

1.6.2 Additional Biological Matrices ......................................................................19

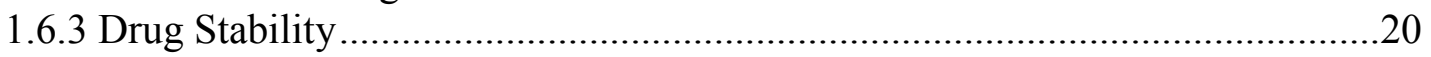

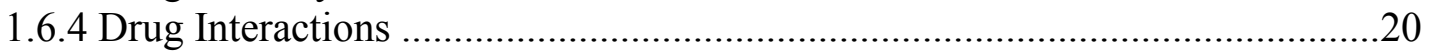

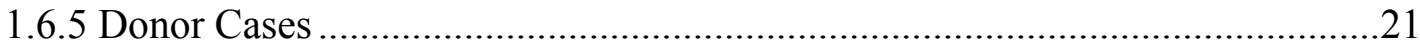

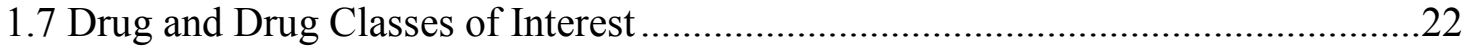

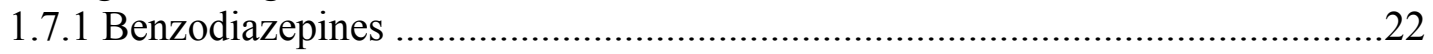

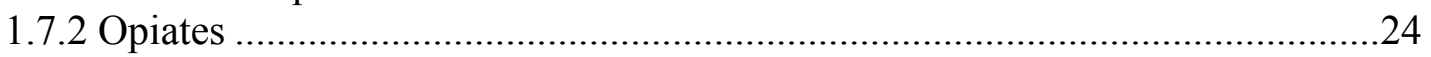

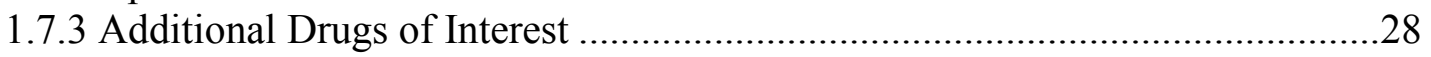

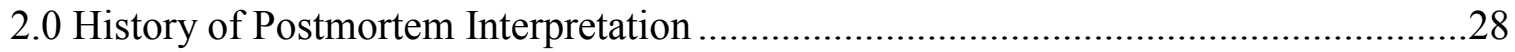

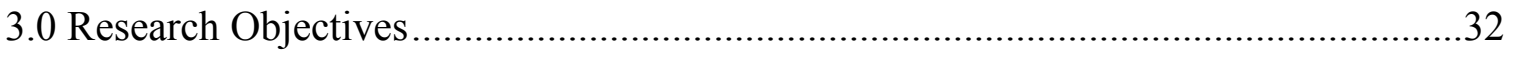

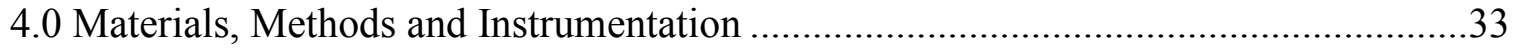

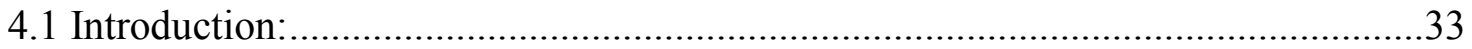

4.2 Benzodiazepines: Quantitative Blood Analysis by GC/ECD, HPLC/DAD

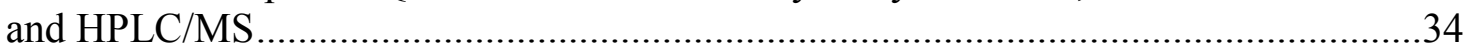

4.2.1(a) Standards and Extraction Procedure for GC/ECD and HPLC/MS...............35

4.2.1 (b) Standards and Extraction Procedure for HPLC/DAD ..................................36

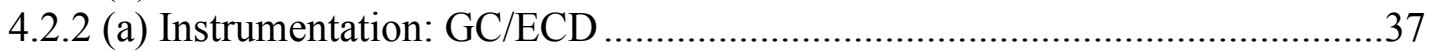

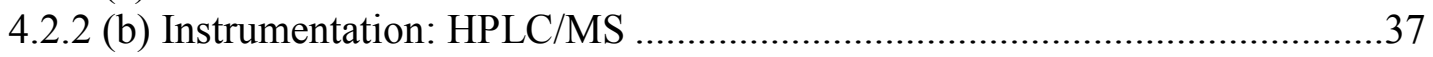

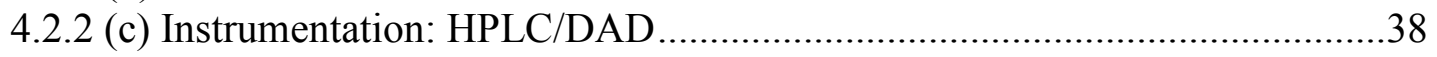

4.3 Basic Drugs: Quantitative Blood Analysis by GC/NPD ........................................39

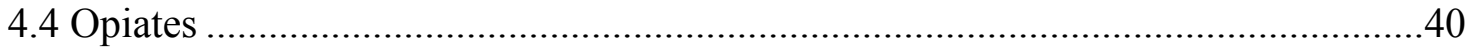

4.4.1 Preparation of Standards for Opiates by GC/MS (SIM) ................................40

4.4.2 Extraction Procedure for Opiates by GC/MS (SIM) ...........................................41

4.4.2 (a) Extraction: Opiate I by GC/MS (SIM) ................................................ 41

4.4.2 (b) Extraction: Opiate II by GC/MS (SIM)....................................................41

4.4.2 (c) Automated Extraction: Opiates I and II by GC/MS (SIM) ........................42

4.4.3 Derivatization: Opiates I and II by GC/MS (SIM) ....................................42 
4.4.4 Instrumentation: Opiates by GC/SIM-MS ..................................................43

4.4.5 Opiates: Quantitative Blood Analysis by GC/MS/MS ..................................43

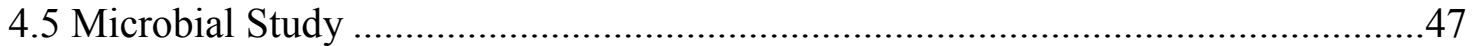

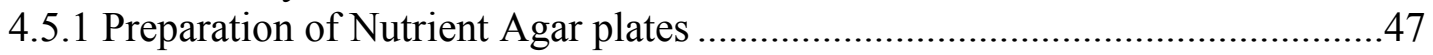

4.5.2 Phosphate Buffered Saline Standards ..........................................................47

4.5.3 Aerobic and Anaerobic Microbial Experiments ..........................................48

4.5.4 Assessing the Usefulness of Codeine and Hydrocodone as a Food

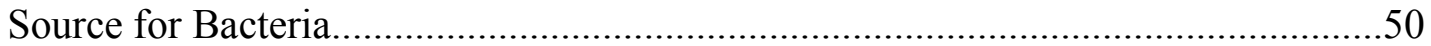

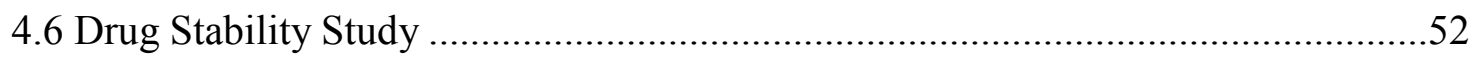

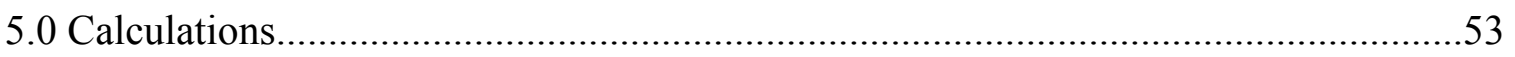

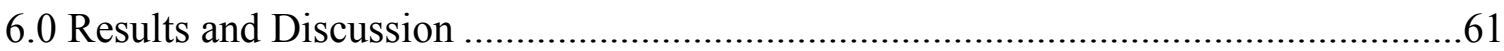

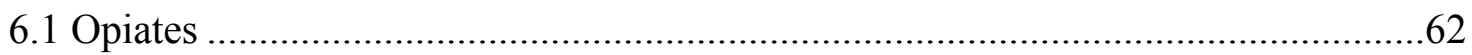

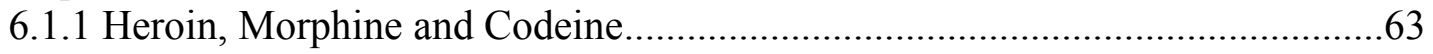

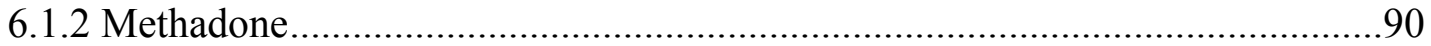

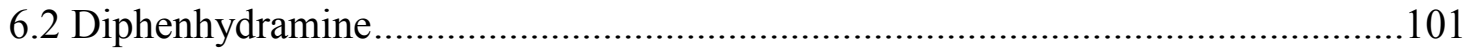

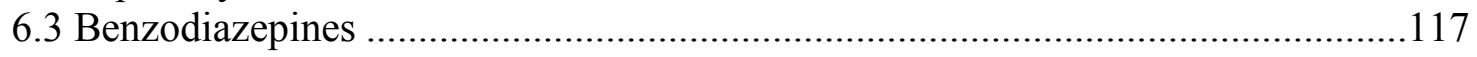

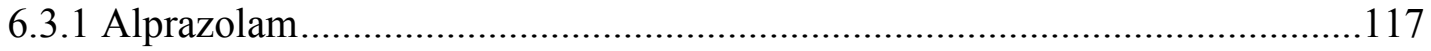

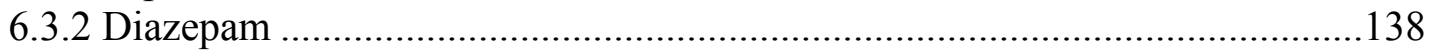

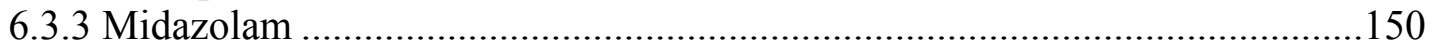

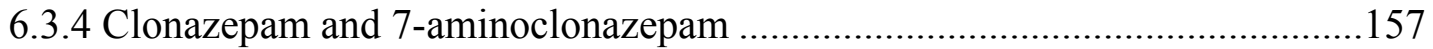

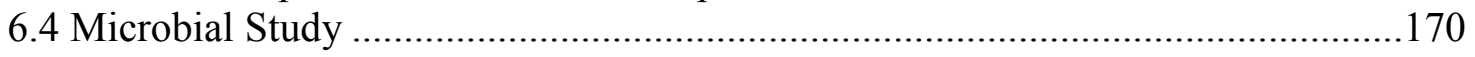

6.4.1 Codeine and Hydrocodone as a Food Source for Bacteria ............................171

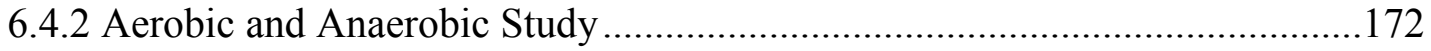

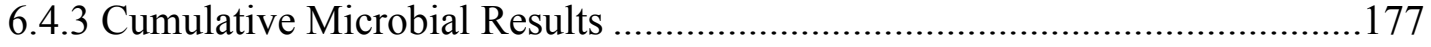

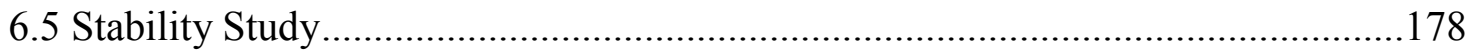

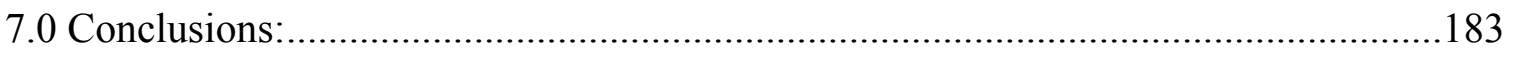

7.1 Microbes and Stability: Potential Influences on Postmortem

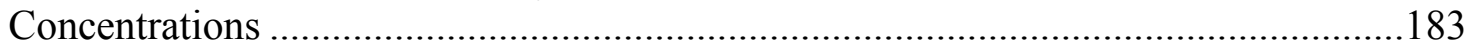

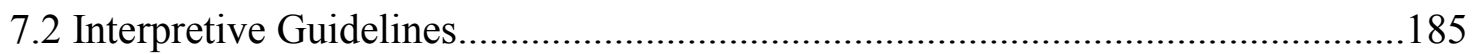

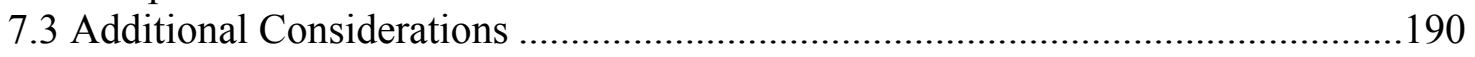

7.4 Final Thoughts and Future Work ...................................................................190

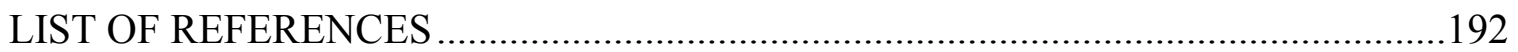

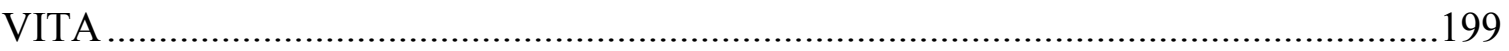




\section{LIST OF TABLES}

TABLE

PAGE

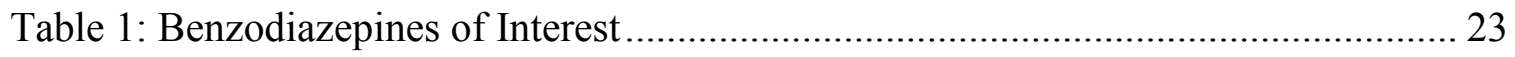

Table 2: Opiates Evaluated for Research .............................................................. 26

Table 3: Benzodiazepines monitored by HPLC/MS/MS ........................................... 38

Table 4: Opiate Compounds Quantitated Using GC/ SIM- MS .................................. 45

Table 5: Opiate Compounds Quantitated Using GC/MS/MS ..................................... 46

Table 6: Example of One-WAY ANOVA Data Output by Microsoft Exel ${ }^{\circledR}$ Generated when Comparing the Three Diphenhydramine Control Lots ................................. 58

Table 7: Two-Way Analysis of Variation Results for Morphine, Codeine and 6-MAM by Comparing Control Data for GC/SIM-MS to GC/MS/MS .............................. 65

Table 8: Coefficient of Variation Results for Morphine, Codeine and 6-MAM ............. 65

Table 9: Morphine, Codeine and Heroin Antemortem samples collected within 2 elimination half-lives

Table 10: Morphine, Codeine and Heroin Antemortem samples collected after 2 elimination half-lives

Table 11: One-Way ANOVA Summary Results for Methadone Controls (0.50 mg/L) .. 92

Table 12: One-Way ANOVA Results for Methadone Controls 92

Table 13: Methadone Antemortem samples collected within one elimination half-life... 96

Table 14: Methadone Antemortem samples collected after one elimination half-life.... 100

Table 15: One-Way ANOVA Summary Results for Diphenhydramine Controls $(0.5 \mathrm{mg} / \mathrm{L})$.

Table 16: One-Way ANOVA Results for Diphenhydramine Controls 104

Table 17: Diphenhydramine antemortem samples collected within two elimination half-lives 106 
Table 18: Diphenhydramine antemortem samples collected after two elimination half-lives

Table 19: Two-Way Analysis of Variation for Alprazolam Comparison of GC/ECD versus HPLC/MS/MS Controls

Table 20: Alprazolam antemortem samples collected within two elimination halflives.....

Table 21: Alprazolam antemortem samples collected after two elimination half-lives . 131

Table 22: Two-Way Analysis of Variation for Nordiazepam Comparison of HPLC/MS/MS to HPLC/UV Control Data

Table 23: Two-Way Analysis of Variation for Diazepam Comparison of HPLC/MS/MS to HPLC/UV Control Data.

Table 24: Diazepam and Nordiazepam antemortem samples collected within one elimination half-life

Table 25: Midazolam Control Data (0.1 mg/L) Statistics.

Table 26: Midazolam Cases listed in order of increasing AM to death intervals 153

Table 27: Two-Way Analysis of Variation for Clonazepam Comparison of GC/ECD to $\mathrm{LC} / \mathrm{MS} / \mathrm{MS}$ control Data.

Table 28: Two-Way Analysis of Variation for 7-aminoclonazepam Comparison $\mathrm{GC} / \mathrm{ECD}$ to LC/MS/MS control Data

Table 29: Coefficient of Variation for Clonazepam and 7-aminoclonazepam 160

Table 30: Clonazepam antemortem samples collected within two elimination halflives 162

Table 31: Clonazepam cases listed in order of increasing postmortem interval. 168

Table 32: Growth Activity of Preserved Blood Stored in an Aerobic Environment...... 176

Table 33: Growth Activity of Preserved Blood Stored in an Anaerobic Environment .. 176

Table 34: Results of the 60 Day Drug Stability Study of Refrigerated Blood 179 


\section{LIST OF FIGURES}

FIGURE

PAGE

Figure 1: Dose-Response Curves for Drug A and Drug B ....................................... 6

Figure 2: Drug and Receptor Relationship ......................................................... 7

Figure 3: Cellular Response as a Result of Drug and Receptor Binding ......................... 8

Figure 4: Acetylation Reaction of Morphine and Codeine ......................................... 27

Figure 5: Arrangement of the 96 Well MT Plate......................................................... 51 


\section{LIST OF CHARTS}

CHART

PAGE

Chart 1: Average Monthly Temperature in Miami, FL from January 2004 - December 2008

Chart 2: Average Monthly Precipitation in Miami, FL from January 2004 - December 2008

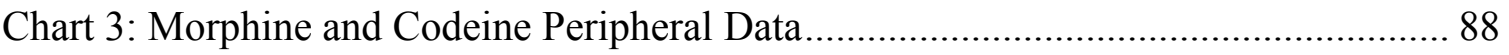

Chart 4: Morphine Central versus Peripheral Data..................................................... 89

Chart 5: Codeine Central versus Peripheral Data .................................................. 89

Chart 6: Diphenhydramine Central versus Peripheral Data........................................ 115

Chart 7: Diphenhydramine Peripheral Source Data................................................ 116

Chart 8: Alprazolam Central versus Peripheral Data................................................ 136

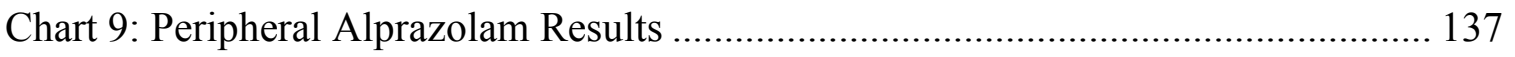

Chart 10: Diazepam and Nordiazepam Central versus Peripheral Data ....................... 148

Chart 11: Diazepam and Nordiazepam Peripheral Data ........................................... 149

Chart 12: Pseudomonas and E. coli Growth Patterns When Using Codeine as a Food

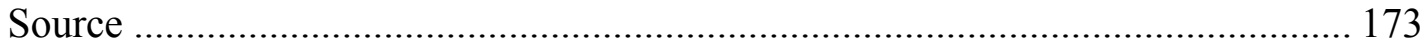

Chart 13: Pseudomonas and E. coli Growth Patterns When Using Hydrocodone as a

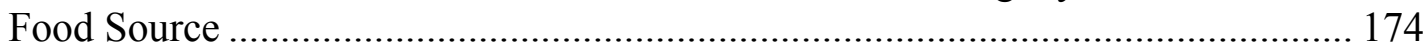




\section{LIST OF EQUATIONS}

EQUATION

PAGE

Equation 1: Apparent Volume of Distribution Calculation ......................................... 18

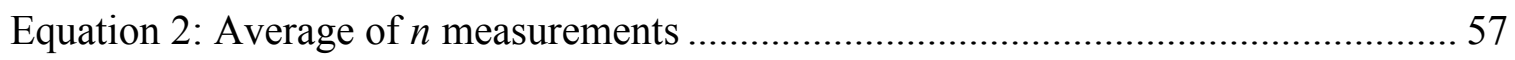

Equation 3: F-test for Comparison of Standard Deviations........................................ 57

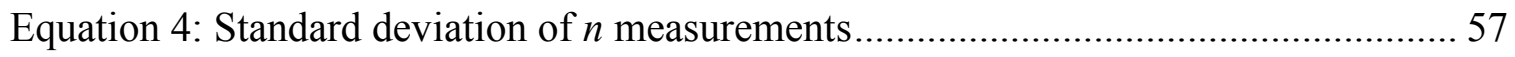

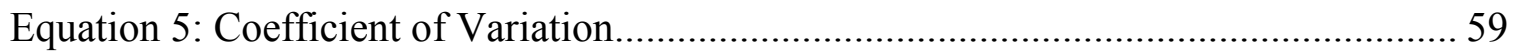

Equation 6: Tukey's Honestly Significant Difference Equation .................................. 59

Equation 7: Calculated Expected Drug Concentration Range at the Time of Death........ 61

Equation 8: Alprazolam's blood to plasma ratio .................................................. 124 


\section{LIST OF ACRONYMS AND ABBREVIATIONS}

Alprazolam

ALP

Antemortem

AM

Antemortem to death.

AMD

Aorta Blood

AOBL

Apparent volume of distribution

$\mathrm{V}_{\mathrm{d}}$

Chest blood

CHBL

Grey top vacutainer tube

GT

Half- life

$t_{1 / 2}$

Heart blood

HTBL

Iliac vein blood

ILBL

Inferior vena cava blood

IVC BL

Limit of detection

LOD

Limit of quantitation

LOQ

Lower limit of quantitation

LLOQ

Methadone

MDN

Miami-Dade County Medical Examiner's Department.

MDME

Mix heart blood.

MXHTBL

Mixed blood

MXBL

Morphine/ Codeine

MOC 


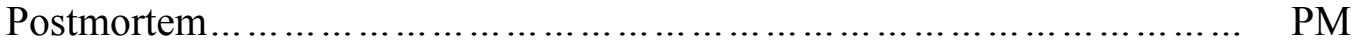

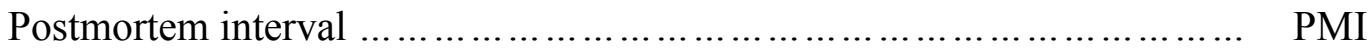

Postmortem redistribution.................................................... PMR

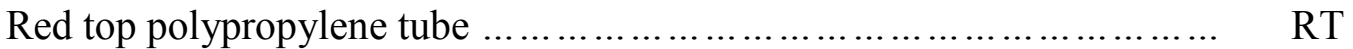

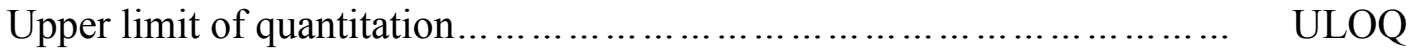




\subsection{Introductory Concepts of Postmortem Toxicology}

\subsection{Medical Examiner Department}

The present study examined cases that fell under the jurisdiction of the MiamiDade County Medical Examiner's (MDME) Department. The MDME is included within the $11^{\text {th }}$ District of the Florida State Judicial System (1). As described by the Florida Department of Law Enforcement (FDLE) - Medical Examiners Commission, Chapter 406 section 11, cases fall within the Medical Examiners jurisdiction when a person dies of violence, accident or suicide. Additional factors include persons of seemingly good health who die suddenly, those in police custody or anyone dying under suspicious or unusual circumstance (2). As part of the medicolegal death investigative process, pathologists conduct autopsies of the body, in order to identify any anatomic reason for death. During this process, biological fluids and tissues are collected and sent for toxicological analyses. The cases utilized for this study were submitted for toxicologic analysis to the MDME - Toxicology Laboratory.

\subsection{Medical Examiner's Toxicology Laboratory}

Biological samples submitted to the laboratory are handled under a chain of custody. The pathologist assigned to the case submits written documentation of each sample (antemortem and postmortem) submitted per case. The collection site for each postmortem blood specimen is specified on the tube and documented within the chain of custody for a given case. Upon receipt of the specimens, laboratory staff members review the submitted documentation, indicate the volume or mass of the specimen 
received and then initial the form. Postmortem blood specimens are received in gray-top vacutainer tubes (total volume $10 \mathrm{~mL}$ ) and in $50 \mathrm{~mL}$ screw cap polypropylene (red-top) centrifuge tubes. Both tubes contain the anticoagulant potassium oxalate and the antimicrobial agent sodium fluoride. In addition to blood, urine, bile, vitreous humor and gastric contents are routinely submitted. Brain and liver specimens are submitted at the discretion of the pathologist. These additional biological matrices are received in vacutainer tubes or plastic containers without preservatives. Upon receipt, all samples are stored in a walk-in refrigerator at $2{ }^{\circ} \mathrm{C}$.

While the toxicology laboratory specializes in postmortem toxicological analyses, antemortem samples from medical examiner cases are also analyzed. Area hospitals provide antemortem (AM) samples to the Medical Examiner Department upon request. Those AM samples collected close to the time of arrival at the hospital are analyzed as a part of routine analysis within the toxicology laboratory. It is most important to analyze arrival specimens as they are reflective of the substances affecting the person when the terminal event was initiated. A specimen referred to as 'delayed', that is those specimens collected during an extended stay in the hospital, can also be obtained upon request. These specimens are analyzed on a case-by-case basis, as they typically only contain medications administered by hospital staff.

Biological fluid and tissue samples, collected at autopsy and area hospitals, are analyzed for the purpose of identifying and quantifying drugs/ toxins within the body. The laboratory first conducts a presumptive screen analysis to identify the presence or absence of drugs or drug classes within each sample. Those substances that were detected in the screen are then submitted for confirmatory testing. Drug confirmation 
conclusively identifies specific drugs (as opposed to classes) found in the screening stage of analysis. However, in keeping with good laboratory practices, the confirmatory technique must be analytically different from that utilized in the screening process. Once the presence of a drug has been confirmed, quantitation may be required. Drug quantitation is the determination of the amount of drug present within a sample. In some instances, quantitation is done at the time of confirmation. In these situations, calibration curves for specific analytes are run as part of the batch analyses. Sample concentrations are then calculated by linear regression. When dual confirmation/ quantitation is not possible, separate quantitative analyses must be prepared. Pathologists submit multiple biological specimens for toxicological analysis. However a peripheral blood sample (venous blood collected from the extremities (e.g. iliac vein)) as opposed to the central cavity (e.g. heart blood) is often the desired specimen for quantitative analysis. The reasons for this will be discussed in greater detail in section 1.6.

The information gathered from toxicological analyses is used in the death investigative process to determine if the presence or absence of exogenous substances contributed to ones death. The MDME- toxicology laboratory uses modern analytical instrumentation including, but not limited to gas and liquid chromatographs, as well as mass spectrometers, electron capture detectors and nitrogen phosphorus detectors (3) to facilitate the identification process. 


\subsection{Forensic Toxicology}

In general terms, toxicology is the study of toxic (or adverse) effects of compounds on biological systems. The field of forensic toxicology works to analyze biological materials for the purpose of identifying toxic substances (including drugs and alcohol) and interpret the results as described by law (4). Forensic toxicologists must maintain objectivity to accurately and justly determine the truth (5).

The three main subsections of forensic toxicology are human-performance, urine drug testing and postmortem. Human-performance forensic toxicology relies on analyses of biological fluids or breath to determine the presence of performance or mood modifying substances. Urine drug testing analyzes urine to determine if drugs and/ or their major metabolites have been used recently. Post-mortem forensic toxicology is the practice of analyzing biological fluid and tissues of deceased persons in order to determine if drugs, chemicals or toxins caused or contributed to death. Since this project focuses on postmortem forensic toxicology, the other subsets will no longer be considered.

\subsection{Pharmacology}

Clinical pharmacology studies in living people have given researchers a strong framework to interpret drug concentrations and their effects. By monitoring both doseresponse and pharmacologic processes, relationships between drug concentrations and effects in humans have been better identified. 
Pharmacological response has two main components, pharmacodynamics and pharmacokinetics. Pharmacodynamics is the study of what a drug does to the body once the target site is reached. Conversely, pharmacokinetics examines how the body absorbs, distributes, metabolizes and eliminates drugs from the system. Both pharmacodynamics and pharmacokinetics are directly related to the dose - response relationship.

\subsubsection{Dose-Response Studies}

Dose-response studies monitor the response an individual or population has to known doses (concentrations) of drugs. Typically, the magnitude of the response is directly related to the given dose. Preliminary dosing experiments involve the use of animals, most commonly mice and rats. In these experiments, the animals are administered known concentrations of drug. The data produced are used to determine the median lethal dose $\left(\mathrm{LD}_{50}\right)$. The $\mathrm{LD}_{50}$ is the dose required to kill $50 \%$ of the population. Once the $\mathrm{LD}_{50}$ is established, animal studies are often extended for a period of years. During this time, side-effects and identification of toxic ranges are determined. Those concentrations determined to be toxic in animal trials can be applied to humans if properly calculated. To do this, the dose per unit of body surface must be measured (6). In order to expand a drug trial to human subjects, it must be determined if the benefits of the drug outweigh the potential risk to human health and safety (e.g. allergic reactions or carcinogenicity). If it can be determined that it does, the drug may be eligible for human testing.

Clinical trials involve the use of human subjects. Since animal trials were used to determine toxic and lethal doses, the primary goal of the human trials is to determine 
which concentrations effectively illicit the desired response. Among other considerations, the data collected from trial participants can be used to determine both drug potency and efficacy. The data can be displayed in a logarithmic curve as seen in Figure 1. In Figure 1 two drugs, $\mathrm{A}$ and $\mathrm{B}$ are displayed. The $\mathrm{x}$-axis is a measurement of drug concentration and is used to measure potency. Drug A is effective at lower concentrations than drug B, making A more potent than B. The $\mathrm{y}$-axis is a measurement of maximum effect. Drug A effects approximately $95 \%$ of the individuals in a trial, while drug B effects only $60 \%$. This makes drug A more effective than B.

Figure 1: Dose-Response Curves for Drug A and Drug B

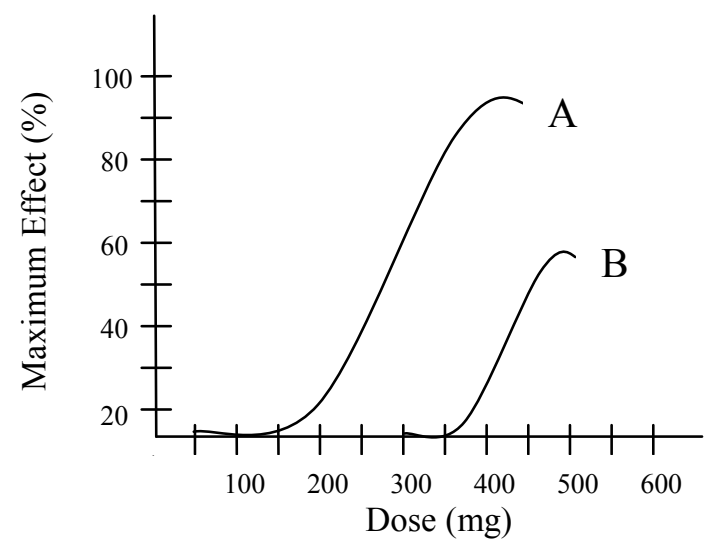

Drug A is both more potent and more effective than drug B 


\subsubsection{Pharmacodynamics}

Pharmacodynamic studies account for both physiological and biochemical effects chemicals impose on a system. In order for a chemical or drug to impose an effect, it must reach its target site and bind to a receptor. As the name suggests, receptors are

Figure 2: Drug and Receptor Relationship

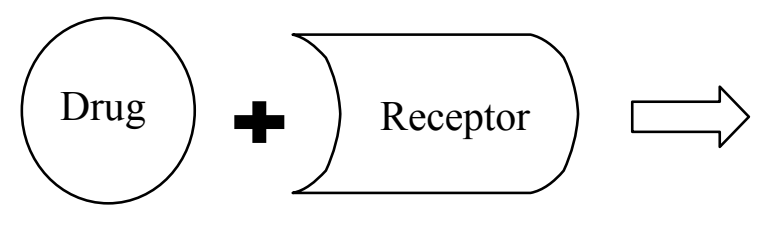

Unbound

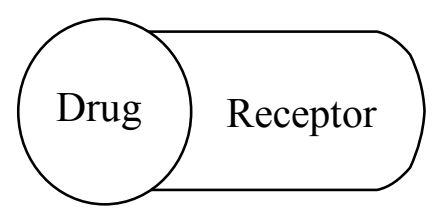

Bound

Upon drug and receptor binding, the drug can impose its effect

substances that are receptive to being bound to chemical agents (7). The combination of drug and receptor binding (Figure 2) determines how a drug will affect the body. As a result, chemical agents have been categorized as agonists, antagonists, partial agonists and inverse agonists.

Agonists are chemicals that, when bound to their receptor sites illicit, a biochemical or physiological response. The given response mimics the effects of endogenous substances. The intensity of the response however, can vary. If the resultant effect does not reach its full intensity, the agonist is referred to as a partial agonist (8). An inverse agonist (antagonist) imposes the opposite effect of the agonist in Figure 3. When an antagonist is bound to a receptor, it inhibits the effects of the agonist by blocking its action. Antagonists are important to the medical community because they can be given 
to counteract the deleterious effects of drugs. Agonists and antagonists can exist in the system at the same time. In those situations, the chemical with the highest affinity to the receptor will be bound. The resultant effect is dependent upon which compound is bound to the receptor.

\section{Figure 3: Cellular Response as a Result of Drug and Receptor Binding}

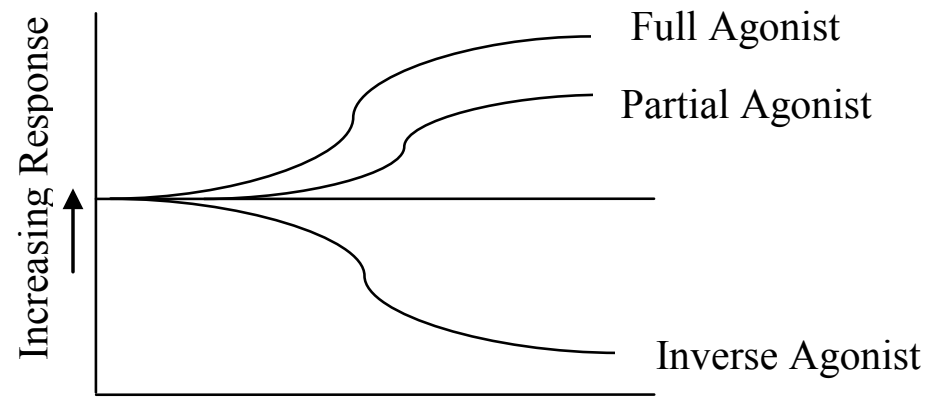

Decreasing Concentration

The partial agonist is imposing the same effect as the full agonist, except with a diminished response. The antagonist (inverse agonist) is exerting the opposite effect of the agonist.

\subsubsection{Pharmacokinetics}

Drugs and other chemical substances can be administered in a multitude of ways, including but not limited to oral ingestion, parenteral injection, inhalation, transdermal absorption or by insufflation. Upon administration, the first task of a chemical substance is to cross the cellular membrane. The membrane is composed of a lipid bilayer, made of hydrophilic heads and hydrophobic tails. In general, chemical compounds can traverse the bilayer either by passive diffusion or active transport. Those chemicals that are small, 
unbound and nonionized have a greater possibility of passively diffusing across the membrane. In passive diffusion, the chemical agent moves in response to a concentration gradient (from high to low) without requiring an expenditure of energy (9). Those molecules that are not amenable to diffusive mechanisms move via active transport. The active translocation of chemicals across the cellular membrane requires energy, partly because the chemicals are moving against the concentration gradient (from low to high) (8). Whether by passive or active transport, once a chemical has crossed the lipid bilayer, it has been absorbed into the system.

After absorption, a drug is able to distribute throughout the body. Typically, highly vascularized tissues (e.g. liver, brain and kidneys) receive the drug first. Muscle, fat and skin are secondary recipients for the drug (10). Chemical compounds preferentially distribute themselves either in the tissues or in the blood stream. The solubility of the drug and the $\mathrm{pH}$ gradient established between the blood and tissues are determining factors. In order to identify the extent to which a drug has distributed itself, the apparent volume of distribution $\left(\mathrm{V}_{\mathrm{d}}\right)$ can be determined. The apparent volume of distribution is the volume of blood into which a drug appears to be distributed. It is not based on the actual volume of fluid present within a person, but rather the volume needed for a drug to be evenly distributed between tissues and organs. It has been argued that the higher the $\mathrm{V}_{\mathrm{d}}$, the more likely a drug is to be sequestered in body tissues rather than remain in circulation. Conversely, those drugs that remain in circulation are susceptible for distribution, metabolism and elimination. During this time, a drug's elimination halflife must be considered. Elimination half-life $\left(t_{1} / 2\right)$ is the amount of time it takes to reduce a drugs concentration by $50 \%$ (11). Some drugs, such as heroin, have a relatively short 
half-life (2-6 minutes), while others like methadone have a more prolonged half-life (1555 hours). Knowing a drug's half-life can be a useful tool in predicting the expected concentration of a drug in blood circulation after a given amount of time.

During metabolism, the body converts foreign materials into polar, water soluble compounds that are easier to eliminate. The liver, the primary organ of metabolism, has a substantial number of enzymes used to aid in this process. Drug metabolism can occur in one of two mechanisms (first pass or post distribution) and in one of two phases.

First pass metabolism primarily occurs in orally administered drugs. After ingestion, the drug can be metabolized in part by the intestines, but primarily by the liver before systemic circulation begins. The metabolism and subsequent elimination of a drug before distribution can significantly decrease its bioavailability. Bioavailability is a term used to describe the remaining amount of drug available to affect the system post ingestion. Bioavailability is an important factor to consider, because if a drug is highly susceptible to first pass elimination, its bioavailability will be significantly reduced. Drugs administered via intravenous injection, sublingual dissolution or insufflation are not affected by first pass metabolism as they directly enter the blood stream and are distributed upon absorption. As a result, they are eliminated post distribution. Whether a drug is eliminated by first pass or post distribution mechanisms, all drugs will eventually be metabolized.

Metabolism occurs in one of two phases. Phase I reactions increase chemical polarity by either adding of modifying functional groups on the compound. Phase I reactions can sufficiently rid the body of foreign materials on its own. However, if the chemical elimination does not occur rapidly enough, a secondary reaction (Phase II) can 
occur. Phase II reactions add endogenous substances (e.g. glucuronic acid, sulfate, acetate or amino acid) to the exogenous chemical to form a conjugate (12). Conjugation greatly increases polarity and water-solubility; thereby facilitating elimination.

Chemical elimination is regulated by the kidney. The kidneys excrete nonvolatile, water soluble, polar compounds in urine. The rate of elimination depends on the chemical properties of the drug as these properties directly affect the speed and efficiency of the excretion process. The phase I and II reactions work to metabolize chemical agents into a readily excretable form. However, there are situations where instead of elimination a drug, is reabsorbed back into the system.

The $\mathrm{pH}$ of the fluid in the kidneys is vital to its ability to eliminate drugs. Therefore, if the $\mathrm{pH}$ of the fluid is slightly basic, acidic compounds will become ionized and eliminated through urine. However, any basic drugs present will remain nonionized allowing them to potentially be reabsorbed back into the body. If reabsorption occurs, the body can again be affected by the drug's presence. However, the body will respond once more by trying to eliminate it metabolically.

Pharmacological studies examine the relationship between physiological and biochemical response, as well as concentration and time. Dose-response analyses measure concentration and response (13). The combination of the information gained from both pharmacology and dose-response research has been used to develop the interpretive framework of toxicology. The data produced in clinical pharmacology studies on living humans have aided in the development of analytical tools (e.g. tables and charts) which can help assess the effects of drugs at known concentrations. This data has been used as a guideline in determining physical, psychological and pharmacological 
effects of drugs. When applying this information to living subjects, these studies have proven useful. However, attempting to apply the same information to postmortem drug concentrations in deceased subjects has led to substantial errors.

\subsection{Postmortem Processes}

Just as living people have body processes, so do deceased persons. However, these processes are significantly different. Upon death, the body no longer receives a flow of blood carrying oxygen and nutrients and removing wastes. As a result, cells begin to die and the process of decomposition has begun.

Human decomposition can begin within five minutes of death and is governed by autolysis. Autolysis, otherwise known as self digestion, is the breakdown of cellular materials by enzymes (14). This process begins within the intestines. The lack of oxygen in the body allows for anaerobic bacteria and enzymes to take over and digest the intestine. As the digestion continues, the enzymes will break out of the intestine and begin digesting the surrounding organs and other body tissues. As the cells within the body die, they release drugs, toxins and other substances.

While the cellular breakdown of the body is not immediately visible, with time it does show itself on the outside of the body in the form of skin blisters and slippage (large pieces of skin separating from the body) (15). In addition to the anaerobic and enzymatic activity, internal changes including body temperature decrease, blood settling in the direction of gravity (causing skin discoloration) and rigor mortis (body stiffening) occur. 
After these initial changes, the body will bloat as a result of the production and build up of gas inside. During bloat, insect activity flourishes, resulting in the acceleration of organ and tissue breakdown. Eventually, the gas is expressed and the body collapses (decay). In addition to gases, the liquefied tissues of the body are released as well. The drainage of the liquids allows the body to dry out and depending on the environmental conditions mummification or skeletonization ensues.

The rate of decomposition can vary from weeks to years, depending on the environment surrounding the body. Climate, precipitation, trauma and insect activity can all contribute to the rate of decomposition (16). In warmer, more humid environments decomposition can be accelerated, compared to cool, dry environments. This is in part the result of increased insect activity, as insects flourish in these types of environments. Maggots are largely responsible for the digestion of body tissues (17). Eggs are deposited into body orifices (e.g.eyes and mouth) as well as areas of traumatic injury, as these open areas are easily accessible. Conversely in situations where the body is buried, encased, wrapped or located in a cold or dry climate, insect activity is diminished, thereby retarding the rate of decomposition (16).

Cases analyzed for this study were obtained in Miami-Dade County in Miami, Florida, USA between the years of 2004 through 2008. Summers (June through September) during those four years in Miami, FL had an average temperature of $82.6^{\circ} \mathrm{F}$ (Chart 1) with an average monthly rainfall of 0.29 inches (18). As a result, the relative humidity in Miami can well exceed $85 \%$ during summer months (19). Winters (December through February) in Miami tend to be milder, with the average monthly 
Chart 1: Average Monthly Temperature in Miami, FL from January 2004 December 2008

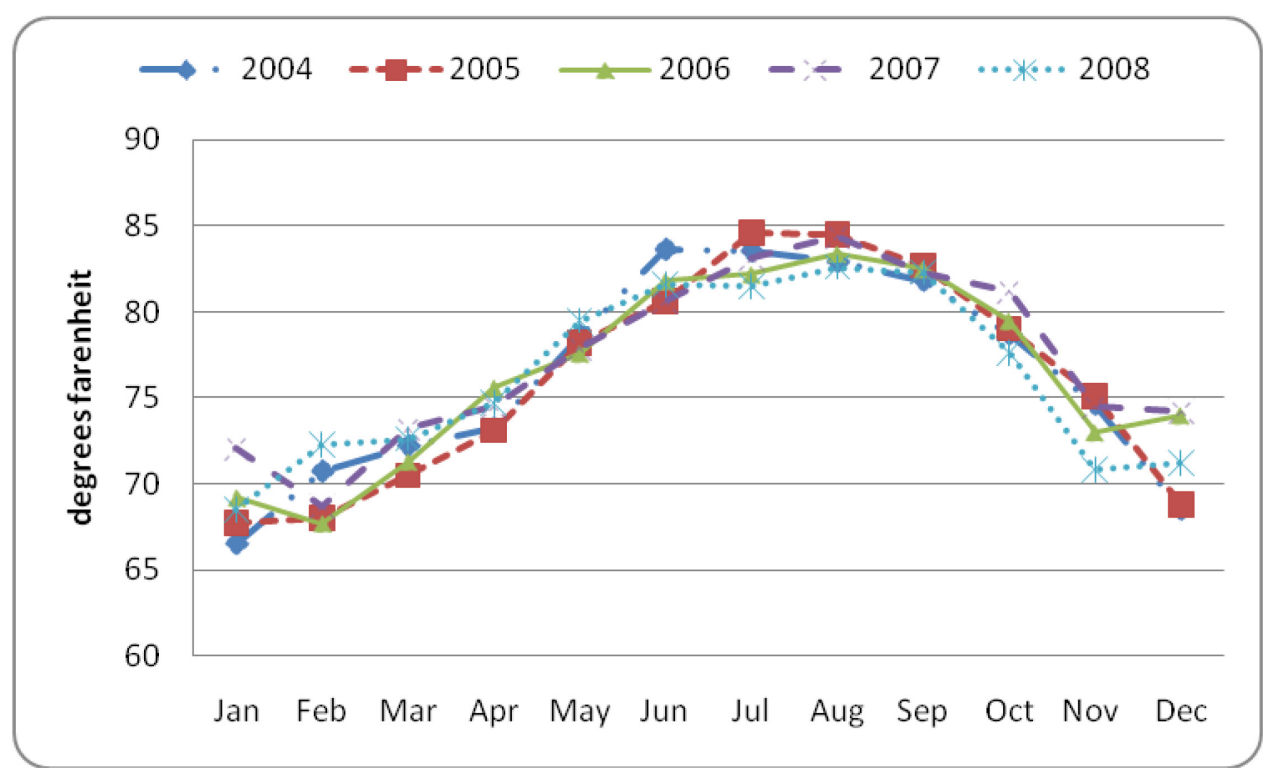

Chart 2: Average Monthly Precipitation in Miami, FL from January 2004 December 2008

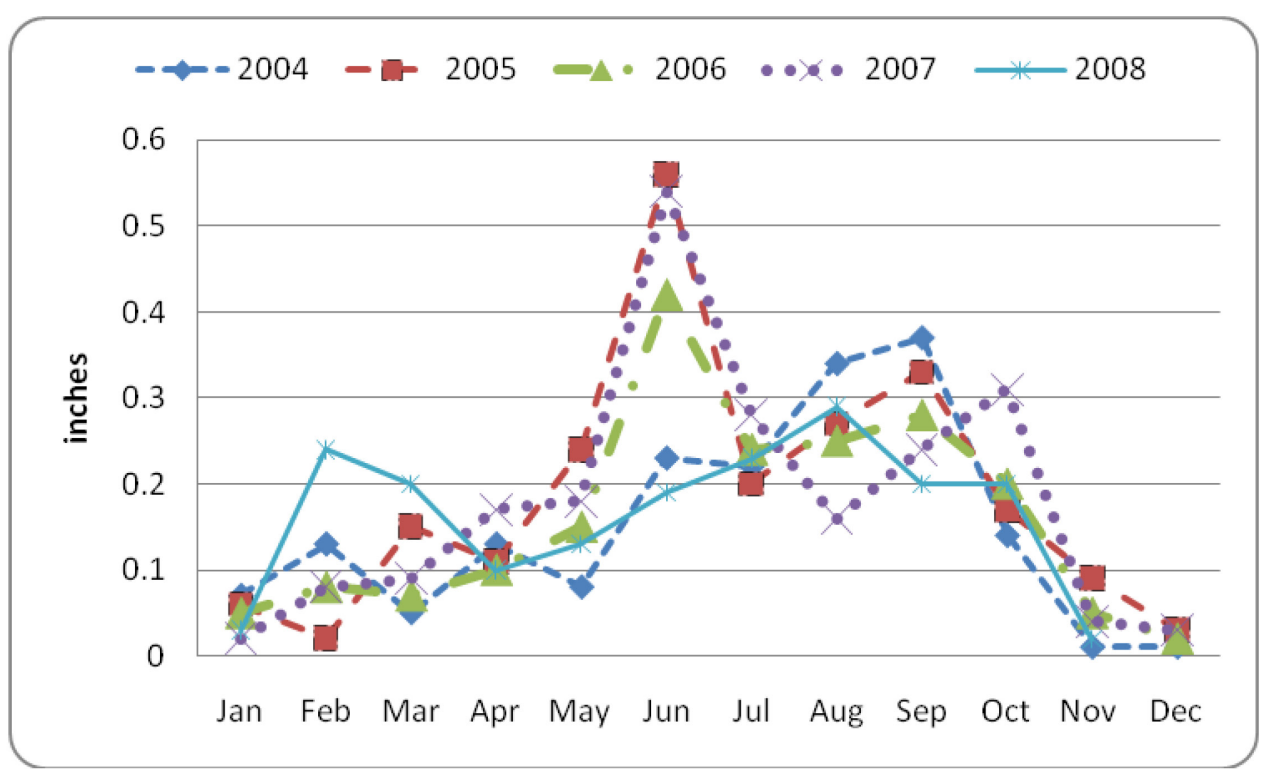


winter temperature and precipitation (2004-2008) being $69.9^{\circ} \mathrm{F}$ and 0.06 inches, respectively (19). The average monthly precipitation for the years $2004-2008$ are displayed in Chart 2.

As a result of the climate, depending on the conditions of the body (e.g. indoors, outdoors or wrapped) advanced decomposition can occur within 24 hours after death. In terms of toxicology, decomposition fluid has very limited value as it is a mixture of degraded tissues and organs. As a result, any quantifiable data obtained is a result of a contaminated sampling pool. Therefore, samples containing decomposition fluid were not used for this study.

The body processes in living people (as discussed in section 1.4 Pharmacology) are vastly different from those processes occurring in deceased persons. Even in the earliest stages of decomposition, the processes of the body are dissimilar to those occurring in a living person. The effects these distinctively different processes have on the body are what make it virtually impossible to apply the same interpretive parameters to both living and deceased people. A thorough guide exists for clinical toxicologists; however, one is still needed for postmortem toxicologists. The lack of an accurate and reliable interpretive framework for PM toxicology has opened the field up to gross errors and mis-steps.

\subsection{Interpretive Considerations of Postmortem Toxicology}

As was discussed in sections 1.4 and 1.5, there are unique considerations for interpreting drug concentrations for both living and deceased persons. One of the goals

of this project was to gain a better understanding of postmortem drug interpretation. In 
doing so, some fundamental concepts must be understood. These include the effect of specimen choice, postmortem interval, postmortem redistribution, and drug stability. All of these factors influence a toxicologist's ability to interpret postmortem drug concentrations.

\subsubsection{Specimen Choice (Whole blood) and Postmortem Phenomena}

In postmortem toxicology, quantitative analysis is usually conducted on whole blood. Those drugs identified in blood samples are believed to be reflective of the substances potentially affecting a decedent at the time of death. This is an extremely important detail when trying to determine if the presence or absence of drugs contributed to ones death.

At autopsy, both central and peripheral blood samples are collected when possible. Central sourced blood is collected from the trunk of the body (e.g. heart or aorta). Blood collected from the body's extremities is considered to be peripheral blood. Common examples of peripheral blood include that from the femoral or iliac veins. Since blood can be collected from any number of areas, the samples submitted for analysis are labeled with the appropriate source. The correct designation of the source is important because a phenomenon known as postmortem redistribution can cause inconsistent quantitative results among blood and tissues collected within the same body.

Postmortem redistribution (PMR) is a term used to describe a complex array of variables that can lead to the redistribution of drugs after death, which in turn can result in quantitative concentration discrepancies. In general, postmortem blood concentrations can vary depending on collection site and the time interval between death and autopsy. 
As a result, postmortem blood concentrations are not believed to be reflective of blood concentrations present at, and around, the time of death. The combination of physiochemical drug characteristics, cellular and tissue breakdown during decomposition, as well as the proximity of internal organs and vessels (20) all contribute to a drug's ability to redistribute itself in the postmortem. Since upon death, the body is no longer able to produce energy, it is believed that passive diffusion of drugs (down the concentration gradient) is responsible for their movement (21).

Postmortem redistribution occurs more extensively in areas of the body where drug storage depots and vessels are in direct contact with each other. Not only are the liver, lungs and gastrointestinal tract well known drug reservoirs (20), but their proximity to each other (as well as vessels) can facilitate a more extensive redistribution effect (22). In contrast, peripheral vessels have limited contact with adipose and skeletal muscle. Both of which are susceptible to the decomposition processes at a much slower rate than the central organs. Therefore, peripheral blood sources, which are believed to be protected from the initial putrefactive processes, are the preferred sampling sites for postmortem quantitative work.

The extent to which a drug is able to redistribute itself is often governed by its apparent volume of distribution $\left(\mathrm{V}_{\mathrm{d}}\right)$, which is mathematically calculated in Equation 1 (23). The $\mathrm{V}_{\mathrm{d}}$ value accounts for drug chemistry as some drugs remain in the vessels (hydrophilic) while others preferentially store themselves within the tissue (lipophilic). Higher values for the $\mathrm{V}_{\mathrm{d}}$ reflect a greater preference of a drug to concentrate itself in the tissues rather than in blood (24). Thus it is believed that drugs with higher volumes of distribution can be released from their storage tissues and diffuse into the vessels and 
surrounding tissues during redistribution. For that reason, blood collected from the major vessels in the central cavity of the body, is more likely to be contaminated by the drug concentrations of the surrounding organs (e.g. liver and lungs).

\section{Equation 1: Apparent Volume of Distribution Calculation}

$$
\mathrm{V}_{\mathrm{d}}=\frac{\text { Amount of drug in the body }}{\text { Concentration of drug in the blood or plasma }}
$$

The result reflects a theoretical volume needed for a drug to evenly distribute between blood and tissue

The extent of PMR can also be influenced by the postmortem interval (PMI). This is the time between death and sampling at autopsy. Greater time intervals result in increased time for drug release and redistribution to occur. Although central blood is not the preferred source for quantitative analysis it is not without value. Typically the volume of the central blood available is far greater than that of peripheral blood. Therefore, central blood can be utilized for initial qualitative analysis, followed by peripheral blood when additional interpretive information is needed (25).

The quantitative differences between central and peripheral sources is known as the central to peripheral $(\mathrm{C}: \mathrm{P})$ ratio (22). As previously mentioned, the central source blood is more prone to redistribution and thus contamination. On the basis of the central to peripheral theory, when redistribution occurs the central sourced blood will have a higher concentration than the periphery, which leads to a C:P ratio greater than 1 . Another ratio used to measure the extent of PMR is the postmortem to antemortem 
(PM:AM) ratio. Similar to the C:P ratio, when redistribution is believed to be present, the PM to AM ratio should be greater than 1. The PM: AM ratio are believed to be more susceptible to the PMI, meaning the greater the interval, the greater the chances of redistribution. While the $\mathrm{C}: \mathrm{P}$ ratios are believed to be more influenced by the anatomical location of the blood sources. Regardless of which ratio is used, PMR in and of itself does not always respond in a predictable manner (26); therefore, neither relationship will always hold true.

\subsubsection{Additional Biological Matrices}

In addition to whole blood, urine, bile, vitreous humor and gastric contents are also routinely collected at autopsy. Urine analysis provides qualitative information about recent drug use. It is not used for quantitative work because the drug content present in urine has already been metabolized and removed from blood circulation. As a result, drugs identified in urine are not reflective of what was actually affecting a decedent around the time of death. Bile typically is used for qualitative analysis but has proven additionally useful as an alternate sample for quantitative ethanol testing (27). The eyes, which are remotely located from the central cavity, work as a protective case for vitreous humor. The location of the vitreous allows for both minimal contamination and bacterial degradation (27) (28). The decreased bacterial fermentation makes vitreous a reliable quantitative sample for ethanol analysis. Additionally, quantitative analysis of gastric

contents can identify drug overdoses and distinguish between accidental and intentional overdose (29). 
When interpreting postmortem drug concentrations the information gained from the above biological matrices aids in developing the full toxicological spectrum of each case. However, the scope of this project focuses solely on the direct comparison between antemortem (AM) and postmortem (PM) blood samples. Additionally, bile, vitreous humor and gastric contents are not potential specimens for collection from living patients.

\subsubsection{Drug Stability}

Upon consumption, some drugs are more unstable than others. Heroin, a synthetic opiate, is rapidly sequentially hydrolyzed to 6-monoacetylmorphine, then to morphine. Both heroin (3,6-diacetylmorphine) and 6-monoacetylmorphine are very unstable in blood with half lives of $2-6$ minutes and $6-25$ minutes, respectively (30).

One study, conducted on all male subjects, found that regardless of ingestion route (intranasal, parenteral or insufflation), heroin concentrations peaked within 5 minutes of ingestion and then fell below $1 \mathrm{ng} / \mathrm{mL}$ detection limits thirty minutes post ingestion (31). Because of the rapid hydrolysis of heroin and 6-monoacteylmorphine, in cases where a fatal heroin overdose is suspected, factors such as the postmortem interval (PMI) and the time between ingestion and death usually preclude an analyst's ability to detect heroin. In such situations, metabolite detection is far more likely.

\subsubsection{Drug Interactions}

When interpreting toxicological data, it is important to consider the total effect of all drugs present (29). While some drugs may not contribute to death, others may impose additive or synergistic effects resulting in a deadly outcome (24). When two or more 
substances additively interact, they produce a total effect that would be equal to the sum of the effects of each drug if given separately. Synergistic interactions have enhanced toxicity as their cumulative effect is greater than would be expected of either substance if given separately. Synergism is often displayed when substances imposing the same effect are taken in combination. For example, narcotic analgesics (e.g. fentanyl), benzodiazepines (e.g. alprazolam) and ethanol can all cause respiratory depression. When taken in combination, fatal results can occur at concentrations both within and below therapeutic levels (24) (32).

\subsubsection{Donor Cases}

Donor cases are those in which the decedent's body was used for organ donation. In these situations, a hospitalized patient must first be pronounced dead. The term 'death' refers to brain death. Up until the time of brain death, a patient may be receiving medications and treatment by the hospital staff to help maintain life. Medications, such as corticosteroids, are given to help reduce the swelling of the brain in order to preserve its integrity and function. A side effect of this treatment is that the organs of the body will dehydrate, thereby compromising their function. However, at the time of brain death the use of such medications are ceased. Organ functions (including the beating of the heart, kidney filtration and liver metabolism) are maintained artificially with the aid of medical equipment. As a result, body processes such as metabolism and urine production are still active. In addition, in preparation for organ procurement, the body is infused with fluids to rehydrate the organs. A donor may receive fluids and be maintained on life support for a matter of hours or even days after brain death. 
Organ harvesting is a surgical procedure that occurs in a sterile, surgical environment. Just prior to the surgery, blood and urine samples are collected. These samples are often obtained by the MDME's toxicology laboratory. However, infusions of hospital fluids severely dilute the concentration of drugs of toxicological importance. Additionally, those drugs that are identified and/ or quantified in donor cases are typically those administered by the hospital. Therefore, their presence may render little insight into the cause of death. As a result, donor samples are obtained and stored, but often not tested as a part of routine analysis.

\subsection{Drug and Drug Classes of Interest}

\subsubsection{Benzodiazepines}

Benzodiazepines are a class of drugs which fall under the category of central nervous system (CNS) depressants. Chlordiazepoxide (Librium ${ }^{\circledR}$ ), the first benzodiazepine, was introduced to the world in the 1960's; diazepam (Valium ${ }^{\circledR}$ ) soon followed. Benzodiazepines were first used as a replacement for barbiturates (a class of sedative hypnotic drugs). Barbiturates, while effective, required higher doses, had more severe withdrawal symptoms and had higher incidence of unintentional overdose than benzodiazepines. The perceived increased safety offered by benzodiazepines along with their wide array of uses (e.g. anti-anxiety, antidepressant and anticonvulsant), has now made them among the most frequently prescribed medications in the United States.

Structurally, all benzodiazepines have the same heterocyclic ring structure, consisting of a benzene ring fused to a 7-member diazepine ring. However, they have 
variations which include 1,4 -benzodiazepines (e.g. diazepam), 7-nitro-1,4 benzodiazepines (e.g. clonazepam) and -azolo benzodiazepines (e.g. midazolam and alprazolam) (33). While the actual effectiveness of benzodiazepines has been questioned in recent years (34), they are still widely prescribed for their sedative, hypnotic and antiepileptic properties. However, studies have suggested that their long-term use can result in cognitive dysfunction, psychomotor impairment and physical dependency (35) (36).

Table 1: Benzodiazepines of Interest

\begin{tabular}{|c|c|c|c|}
\hline Chemical Name & Trade Name & Usage & Duration \\
\hline 7-aminoclonazepam* & N/A & N/A & N/A \\
\hline Midazolam & Versed $^{\circledR}$ & $\begin{array}{l}\text { Sedation, } \\
\text { amnesiac } \\
\text { properties }\end{array}$ & short-acting \\
\hline Nitrazepam ISTD & Mogadon $^{(R)}$ & Sedative & long-acting \\
\hline Clonazepam & Klonopin $^{(B)}$ & Anticonvulsant & long-acting \\
\hline Alprazolam & $\operatorname{Xanax}^{(B)}$ & $\begin{array}{c}\text { Antidepressant } \\
\text { \& Anxiolytic }\end{array}$ & short-acting \\
\hline Nordiazepam & Stilny ${ }^{(B)}$ & Anxiolytic & long-acting \\
\hline Diazepam & Valium $^{(B)}$ & Anxiolytic & long-acting \\
\hline Bromazepam ISTD & Lectopam $^{(B)}$ & Anxiolytic & short-acting \\
\hline
\end{tabular}

Dosing amounts of benzodiazepines are largely reflective of their intended treatment. Generally, high doses are used as hypnotics, moderate doses as anxiolytics and low doses are employed for their sedative properties (37). When taken as prescribed, benzodiazepines rarely cause toxicity. However, it has been well documented that when 
taken in conjunction with other central nervous system depressants, they can be highly toxic (24) (32). Additionally, while some benzodiazepines are metabolites, they may still be pharmacologically active. For example the parent drug diazepam $\left(\right.$ Valium $^{\circledR}$ ) can metabolize to nordiazepam $\left(\right.$ Chlorazepate $\left.^{\circledR}\right)$, temazepam (Restoril ${ }^{\circledR}$ ) and oxazepam $\left(\operatorname{Serax}^{\circledR}\right)$. All four drugs are available by prescription as they all effectively treat anxiety. As a result, blood analysis for this class of drugs can be rather extensive. Table 1 outlines the benzodiazepines analyzed for this study.

\subsubsection{Opiates}

Opiates are a class of drugs derived (either naturally or synthetically) from opium poppy. Opiates are often referred to as narcotic analgesics as these highly addictive medications are a potent remedy for severe pain. While opiates are the most effective pain management medication available, they offer many undesirable effects including stupor, nausea, vomiting and respiratory depression. Opiate users (both licit and illicit) often build a tolerance to the medication, which can lead to abuse. In terms of toxicology, tolerance makes it difficult to properly interpret the significance of opiate concentrations in blood (21). For this study, hydrocodone, codeine, oxycodone, morphine, 6-monoacetylmorphine (6-MAM), hydromorphone and oxymorphone were monitored. However, a sufficient amount of case data was only collected for morphine, codeine and 6-MAM. Their results are discussed in the 6.1 Opiates section. As a result of the lack of data, oxycodone and oxymorphone were no longer considered. However, hydrocodone and hydromorphone are still included in the results section as they were 
monitored in the stability experiment (refer to section 6.5). Additionally, hydrocodone (in addition to codeine) was used in the microbial study (refer to section 6.4) as well.

Codeine and morphine each have their own analgesic properties; however, 6monoacetylmorphine is a major metabolite of heroin (3,6-diacetylmorphine) degradation. Heroin is not a naturally occurring opiate; rather it is synthesized from opium poppy. The poppy seeds, which are harvested from an opium plant, are sliced in order to allow their fluid to drain. Contained within this fluid mixture are the naturally occurring opiates, codeine and morphine. A non-specific extraction process is used to isolate both codeine and morphine from the fluid. The compounds are treated with an acetylating agent, which when reacting with morphine produces heroin (3,6-diacetlymorphine). Codeine will react as well, resulting in the formation of the impurity acetylcodeine (38) (39). Upon ingestion, heroin is rapidly converted to 6-MAM, then to morphine, while acetylcodeine is converted to codeine. As a result, heroin usage often results in the detection of 6-MAM, morphine and codeine. While heroin is currently a Schedule I drug (meaning it has no medicinal use in the United States (40)), it was once used to treat tuberculosis and cure morphine addiction (38). However, its addictive nature caused the drug to be banned in the United States in 1956 (38). Heroin screening is typically not part of routine analytical analyses as it is highly unstable in blood (refer to Drug Stability 1.6.3).

While codeine and morphine can be used as markers of heroin abuse, they are also medications in their own right. Codeine can be found in numerous formulations, including prescription cold remedies and antihistamines. Morphine is the primary active ingredient in opium. It is commonly used for pain management and in combination for 
preoperative sedation. Its pain relieving ability is the standard used to measure the effectiveness of newer analgesics (41). Table 2 lists the opiates evaluated in this study.

Table 2: Opiates Evaluated for Research

\begin{tabular}{|c|c|c|c|c|}
\hline Chemical Name & Trade Name & Usage & Treatment & Origin \\
\hline Heroin $^{1,2}$ & N/A & Illicit & N/A & $\begin{array}{r}\text { Semi- } \\
\text { synthetic }\end{array}$ \\
\hline Codeine & Codeine & Antitussive & - & Natural \\
\hline Morphine & Kadian $^{\circledR}$ & Analgesic & $\begin{array}{l}\text { Moderate to } \\
\text { severe pain }\end{array}$ & Natural \\
\hline 6-Monoacetylmorphine ${ }^{2}$ & N/A & N/A & N/A & $\begin{array}{c}\text { Semi- } \\
\text { synthetic }\end{array}$ \\
\hline Hydrocodone & $\begin{array}{c}\operatorname{Vicodin}^{\circledR} \\
\operatorname{Narco}^{\circledR}\end{array}$ & $\begin{array}{c}\text { Antitussive } \\
\& \\
\text { Analgesic }\end{array}$ & $\begin{array}{l}\text { Moderate } \\
\text { pain }\end{array}$ & $\begin{array}{l}\text { Semi- } \\
\text { synthetic }\end{array}$ \\
\hline Hydromorphone & Diluadid $^{\circledR}$ & $\begin{array}{c}\text { Antitussive } \\
\& \\
\text { Analgesic }\end{array}$ & $\begin{array}{l}\text { Moderate to } \\
\text { severe pain }\end{array}$ & $\begin{array}{l}\text { Semi- } \\
\text { synthetic }\end{array}$ \\
\hline
\end{tabular}

${ }^{1}$ Heroin detection was qualitative only

${ }^{2}$ Not approved for medicinal usage 
Figure 4: Acetylation Reaction of Morphine and Codeine

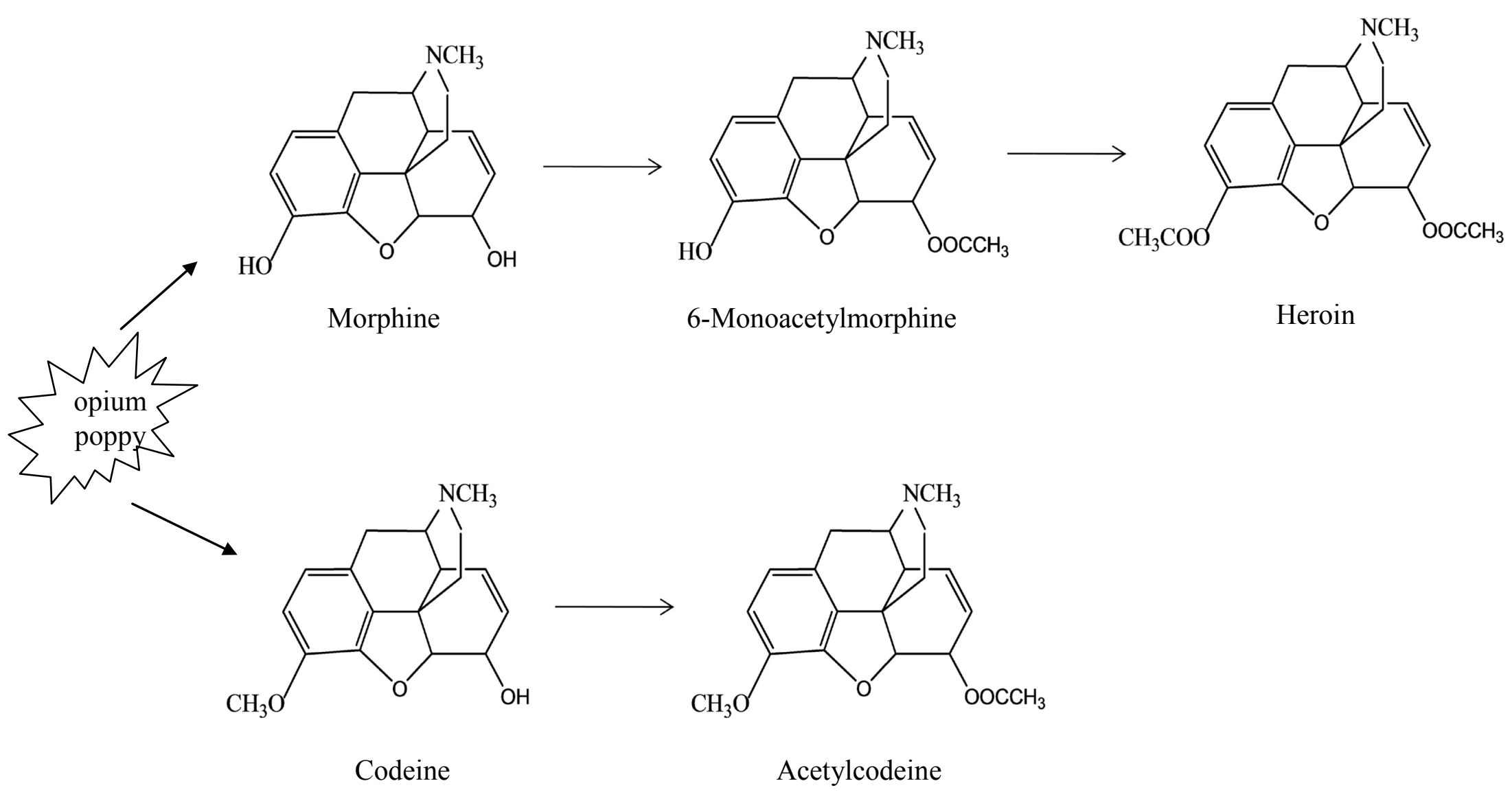




\subsubsection{Additional Drugs of Interest}

Diphenhydramine and methadone were of additional interest for this project. Diphenhydramine is a common over the counter medication. Its properties relieve itchy and watery eyes as well as other allergy related symptoms (Benadryl ${ }^{\mathbb{R}}$ ). In addition, it imposes sedation and therefore has been used as a sleep aid in a number of formulations. Methadone (Dolophine ${ }^{\circledR}$ ) has analgesic properties equal to that of morphine. While it is a central nervous system depressant and works on the same receptors as the opiates, structurally it is not related to them. Continued use of methadone can result in drug accumulation in the system, which can lead to severe sedation (42). Therefore, methadone usage is controlled and almost exclusively used to treat narcotic addiction.

\section{$\underline{\text { 2.0 History of Postmortem Interpretation }}$}

Clinical toxicology is a broad field that encompasses the pharmacokinetic, pharmacodynamic and dose-response relationship that occurs between people and exogenous substances. As a result of understanding these relationships, individualized interpretive guidelines have been developed to identify both physiological and psychological effects of a given drug at a known dosage. The guidelines evolved into a clinical therapeutic index, which is used to identify therapeutic, toxic and lethal dosages (1). For many years, it was these clinical models that were used as the basis of drug interpretation in the field of postmortem toxicology.

A postmortem toxicologist, just as a clinician, would compare drug levels to a clinical model and make a determination about how said drug(s) influenced a person at 
the time of one's death. While clinical models have been invaluable tools for clinicians, they proved to be horribly inaccurate in postmortem work (2) (3) (4) (5). Deceased persons do not undergo the same biological processes as living persons. Consequently, the comparison of postmortem concentrations to clinical models resulted in large errors. However, in the early stages of postmortem interpretation this was not known. Sporadic reports of drug concentration discrepancies began to emerge in the 1970's. Nevertheless, it was not until the late 1980's when the concept of postmortem concentration change came to the forefront of many discussions.

One of the most popular prescription medications in the 1970's was digoxin. Digoxin replaced digitoxin as the primary treatment of heart failure related conditions. With the use of digoxin came several peer reviewed publications detailing analytical techniques for the appropriate extraction and identification of the drug (6) (7). In addition some authors encouraged postmortem interpretation to be conducted on heart serum. As it was believed by them, that heart serum most accurately reflected antemortem concentrations at the time of death (8) (9) (10). Factors that could contribute to PM concentration changes (e.g. the apparent volume of distribution, antemortem to death interval and postmortem interval) were not thoroughly investigated at that time. However, in 1977 Vorpahl and Coe (11) published a study where they aimed to determine if discrepancies existed between antemortem and postmortem digoxin samples. In addition they considered both the influence of the postmortem interval as well as the role of the postmortem collection site on digoxin levels. Ultimately, they determined that significant variations can exist between AM and PM digoxin concentrations. However the length of the PMI could not be correlated to the magnitude of change within the PM 
sample. While Vorpahl and Coe published a fairly comprehensive study on digoxin, this type of evaluation of other drugs was still lacking.

From the late 1970's into the 1980's scientific conferences became the forum in which postmortem toxicologists could discuss their interpretive issues. Many early published articles detailing challenges stemming from postmortem change, by then referred to as postmortem redistribution, referenced talks presented at scientific conferences (12) (13) (14) (15) (16), rather than published sources. With time and concurrence among the postmortem toxicology community, more articles emerged addressing the concentration inconsistencies among drugs such as tricyclic antidepressants (e.g. amitriptyline), ethyl alcohol, propoxyphene and methadone (17) (18) (19). The reporting mechanism however was varied by author. For instance a single case was published by Jones and Pounder (20), in which they analyzed a number of tissues, as well as cerebral spinal fluid, vitreous humor, arterial and venous blood concentrations of 6 analytes. Other authors chose to do compilation studies, where they presented data that had been collected over a period of years (18) (17) (21). Compilation studies typically had an extensive amount of data presented in the form of tables. Some studies presented both antemortem and postmortem results, while others focused solely on various postmortem specimens. During that time ratio calculations, including PM:AM, C:P as well as liver: blood (11) (22) were conducted as a means to identify the source of the discrepancy.

By the 1990's a substantial amount of meetings and publications resulted in a shift in the interpretive process for postmortem work. Toxicologists were now clearly aware of concentration discrepancies that could exist between postmortem and 
antemortem samples. The community strongly advised against using clinical models for interpretation. The only exception to the elimination of the clinical models was the role of drug interaction and how those interactions could have affected someone around the time of their death. Postmortem studies were indicating that certain drugs, particularly those with a high apparent volume of distribution, could disproportionately increase in concentration after death. Factors such as the antemortem to death interval, postmortem interval and ratio calculations were being reported more frequently. However, comprehensive articles about how all of these (and other) factors contributed to postmortem changes were still infrequent. The goal of this project was not to interpret postmortem results, but rather to gain a better understanding for which factors can, and frequently do, influence postmortem results. This comprehensive study was the first of its kind in which the role of microbial activity, drug stability in storage, antemortem intervals, postmortem intervals, postmortem specimen choice, apparent volume of distribution, postmortem to antemortem, as well as central to peripheral ratios were considered for seven commonly encountered medications in postmortem work. The end result both refuted and confirmed some ideals that are currently still in practice. This study also identified the specific variables that have the highest likelihood of influencing each of the seven drugs of interest. While understanding the dynamics of postmortem work may never be as simple as comparing a result to an individualized model, this study has certainly contributed to the understanding of postmortem change and by extension, the interpretation of postmortem results. 


\section{$\underline{\text { 3.0 Research Objectives }}$}

Cases included in this project were submitted with both antemortem (AM) and postmortem (PM) blood specimens. The ideal case had an AM to death interval that did not exceed 24 hours, a postmortem interval within 48 hours and tested positive for at least one drug of interest (refer to section 1.7 Drug and Drug Classes of Interest). Cases that both met and exceeded the time frame requirements were analyzed and the importance of time intervals was evaluated on a drug-by-drug basis in section 6.0 Results and Discussion.

The first objective was to evaluate the differences in drug concentrations between antemortem and postmortem blood. In order to achieve this, data comparisons between intra-case antemortem and postmortem quantitative results were conducted. Whenever available, both postmortem central and peripheral bloods were tested and the impact of postmortem specimen choice was examined. The cumulative data collected for each drug was used to identify concentration trends and ultimately make determinations about drug relationships.

The second objective was to determine how sample preservation impacts drug stability. While the postmortem samples analyzed were preserved in tubes containing the anti-microbial agent sodium fluoride, it was important to assess the effectiveness of the

preservative. To that end, a two-part microbial experiment was conducted. One experiment evaluated the ability of microbes to use drugs as a food source, in the absence of a preservative. The other tested the effectiveness of sodium fluoride under both aerobic and anaerobic conditions. 
Thirdly, it was necessary to assess the stability of drugs after two months of refrigerated storage. The samples analyzed for this study often had a two month (or greater) lapse between collection and analysis. As a result, it was necessary to determine if storage conditions could contribute to concentration discrepancies between antemortem and postmortem samples.

The final objective was to combine the information gained from the first three objectives and develop an interpretive framework for postmortem concentrations. Unfortunately, a set guidelines aiding in the postmortem interpretive process do not exist. The creation of one could be an extremely valuable tool, which could ultimately lead to the reduction of erroneous interpretive errors.

\subsection{Materials, Methods and Instrumentation}

\subsection{Introduction:}

All of the cases used in this project were initially screened and confirmed, as part of the routine analysis by the toxicology staff at the Miami-Dade County Medical Examiner (MDME) Department. The laboratory adheres to strict standard operating procedures (SOPs). All methods were validated prior to their use for case work. In some instances, the initial quantitative data was also collected by the lab staff as part of the death investigative process. This will be discussed in more detail in the results section. The following quantitative methods are those used within the MDME toxicology laboratory and in this project. 


\subsection{Benzodiazepines: Quantitative Blood Analysis by GC/ECD, HPLC/DAD and HPLC/MS}

Benzodiazepine quantitation was conducted on three instruments, gas chromatography/ electron capture detector (GC/ECD), high performance liquid chromatography/ diode-array detection (HPLC/DAD) and high performance liquid chromatography/ mass spectroscopy (HPLC/MS). All data collected as part of routine laboratory analyses were obtained from both the GC/ECD and HPLC/DAD. The GC/ECD was utilized by the researcher for benzodiazepine quantitation until March 2008. After which, quantitative research testing was conducted solely on Thermo Finnigan's LCQ Advantage HPLC/MS system.

The HPLC/DAD system was used to quantitate diazepam and nordiazepam (among other benzodiazepines) with a linear range of $0.1-10 \mathrm{mg} / \mathrm{L}$. The GC/ECD system had a quantitation method for alprazolam, clonazepam and 7-aminoclonazepam with a linear range of $0.01-0.2 \mathrm{mg} / \mathrm{L}$. In an effort to combine the two methods, the HPLC/MS was used to analyze the most frequently encountered benzodiazepines seen in this research project. Those included midazolam, lorazepam, clonazepam, 7aminoclonazepam, diazepam, nordiazepam and alprazolam (linear range: $0.01-1.0$ $\mathrm{mg} / \mathrm{L}$ ). The combination of the extended linear range and the four additional analytes made the HPLC/MS method a convenient alternative, which offered the same level of sensitivity and accuracy as the GC/ECD and HPLC/DAD. Liquid-liquid extraction procedure was used for both the GC/ECD and HPLC/MS systems (Section 4.2.1 (a)), while solid-phase extraction (4.2.1 (b)) was utilized for the benzodiazepines analyzed by HPLC/DAD. 


\subsection{1 (a) Standards and Extraction Procedure for GC/ECD and HPLC/MS}

Stock standards, obtained from Cerilliant Corporation (Round Rock, TX), were used to prepare working standards. The working standards were serially diluted to comprise a five point calibration curve, ranging from $0.01 \mathrm{mg} / \mathrm{L}$ to $0.2 \mathrm{mg} / \mathrm{L}$ by GC/ECD and a six-point curve ranging from $0.01 \mathrm{mg} / \mathrm{L}$ to $1.0 \mathrm{mg} / \mathrm{L}$ by HPLC/MS. After preparation, the standards were spiked in drug-free whole blood. Whenever necessary, samples that exceeded the limits of quantitation were diluted, to bring their concentrations within range, and reanalyzed on the appropriate instrument. Additional stock standards were purchased from Alltech Associates (State College, PA) to prepare controls. A $0.05 \mathrm{mg} / \mathrm{L}$ working control was prepared for $\mathrm{GC} / \mathrm{ECD}$ analysis, while $0.1 \mathrm{mg} / \mathrm{L}$ control was prepared for HPLC/MS analysis. All standard and sample concentrations were calculated using linear regression.

To each $1 \mathrm{~mL}$ blood sample, $100 \mu \mathrm{L}$ of internal standard $(\mathrm{GC} / \mathrm{ECD}=0.4 \mathrm{mg} / \mathrm{L}$ of bromazepam; HPLC/MS $=4 \mathrm{mg} / \mathrm{L}$ of nitrazepam) followed by $1 \mathrm{~mL}$ of $\mathrm{pH} 9$ saturated sodium borate buffer were added. The samples were vortex-mixed and $10 \mathrm{~mL}$ of $\mathrm{n}$-butyl chloride was added. The mixture was capped and rotated for 15 minutes followed by centrifugation (3000 RPM) for 5 minutes. The organic fraction was transferred to a clean tube and the solvent was evaporated at $40{ }^{\circ} \mathrm{C}$ under a stream of nitrogen. The dried extracts were reconstituted with $50 \mu \mathrm{L}$ of toluene (GC/ECD) or $50 \mu \mathrm{L}$ of mobile phase (HPLC/MS) and aliquoted into autosampler vials. 


\subsection{1 (b) Standards and Extraction Procedure for HPLC/DAD}

Stock standards, obtained from Cerilliant Corporation (Round Rock, TX), were used to prepare the calibration standards. The calibration standards were serially diluted to comprise a four point calibration curve, ranging from $0.1 \mathrm{mg} / \mathrm{L}$ to $10 \mathrm{mg} / \mathrm{L}$. After preparation, $100 \mu \mathrm{L}$ of each standard was spiked into drug-free whole blood. Whenever necessary, samples that exceeded the limits of quantitation were diluted to bring their concentrations within range, and reanalyzed. Additional stock standards were purchased from Alltech Associates (State College, PA) to prepare a $1.0 \mathrm{mg} / \mathrm{L}$ working control, containing both diazepam and nordiazepam. All standard and sample concentrations were calculated using linear regression.

To each $1 \mathrm{~mL}$ blood sample, $100 \mu \mathrm{L}$ of nitrazepam internal standard (concentration $=0.1 \mathrm{mg} / \mathrm{L}$ ) was added, followed by $4 \mathrm{~mL}$ of $\mathrm{pH} 6$ sodium phosphate buffer $(0.1 \mathrm{M})$. The samples were vortex-mixed and allowed to stand for 15 minutes. The mixture was then centrifuged ( $3500 \mathrm{RPM}$ ) for 10 minutes. The supernatant was transferred to a clean tube and submitted for automated solid-phase extraction preparation on Caliper Life Sciences (Hopkinton, PA) RapidTrace ${ }^{\mathrm{TM}}$ system.

All solvents used for the rapid trace system were high purity grade. Clean Screen $^{\mathrm{TM}}$ extraction columns (Worldwide Monitoring, Inc.) were first conditioned with an ethyl acetate: ammonia (98:2) elution solvent. Additional conditioning was conducted by the sequential rinsing of the extraction columns with methanol, water and finally $\mathrm{pH} 6$ sodium phosphate buffer $(0.1 \mathrm{M})$. The blood samples were then loaded onto the columns $(2 \mathrm{~mL} / \mathrm{min})$ and then rinsed with water and a $20 \%$ acetonitrile solution. The columns were dried with ultra zero grade air. Three milliliters of elution solvent was passed 
through the column at $1 \mathrm{~mL} / \mathrm{min}$ and the extract collected in a clean screw cap culture tube. In an effort to clean up the extracts, three milliliters of deionized water was added to the eluent. The mixture was rotated for 3 minutes, then centrifuged for 1 minute $($ speed $=3000$ RPM). The organic fraction was transferred to a $16 \times 100$ culture tube and evaporated to dryness at $40{ }^{\circ} \mathrm{C}$ under a stream of nitrogen. The dried extracts were reconstituted with $75 \mu \mathrm{L}$ of mobile phase and aliquoted into autosampler vials.

\subsection{2 (a) Instrumentation: $G C / E C D$}

Agilent technologies HP 6890N network GC model, equipped with dual electron capture detectors and a 7683B series injector was used for quantitative analysis. Two columns (DB-5 and DB-17 both with the following dimensions: $30 \mathrm{~m} \mathrm{X} 0.32 \mathrm{~mm}$ ID X $0.25 \mu \mathrm{m}$ film thickness, Agilent technologies, Palo Alto, CA) were joined at the injection port through the use of a silica "Y" connector. After sample injection, the columns were independent of each other and the flow was carried into their respective detectors. The initial oven temperature of $120^{\circ} \mathrm{C}$ was held for 2 minutes, then ramped at $20^{\circ} \mathrm{C} /$ minute to $290{ }^{\circ} \mathrm{C}$ and held for one minute using nitrogen $(60 \mathrm{cc} / \mathrm{min})$ as the makeup gas. Both

detectors remained constant at $325{ }^{\circ} \mathrm{C}$. Analyte identification was based on relative retention time identification in both columns.

\subsection{2 (b) Instrumentation: $H P L C / M S$}

The samples were analyzed in electrospray ionization (ESI) mode on a Thermo Finnigan LCQ Advantage HPLC/MS system. The narrow-bore Agilent Eclipse - XDB 
C18 column was held constant at $40{ }^{\circ} \mathrm{C}$. Mobile phase, comprised of a mixture of $45 \%$ methanol to $55 \%$ formic acid $(0.1 \%)$ in water, had a flow rate of $400 \mu \mathrm{L} /$ minute. The

Table 3: Benzodiazepines monitored by HPLC/MS/MS

\begin{tabular}{|c|c|c|c|}
\hline Segment & Compound Name & Molecular ion & Qualifying Ions \\
\hline \multirow{2}{*}{1} & 7-aminoclonazepam & 286 & $250 \mathrm{BP}, 222$ \\
\cline { 2 - 4 } & Midazolam & 326 & $291 \mathrm{BP}, 244$ \\
\hline \multirow{2}{*}{2} & Nitrazepam ISTD & 282 & $236 \mathrm{BP}, 254$ \\
\cline { 2 - 4 } & Clonazepam & 316 & $270 \mathrm{BP}, 288,251$ \\
\hline \multirow{2}{*}{3} & Alprazolam & 309 & $281 \mathrm{BP}, 274,251$ \\
\hline 4 & Nordiazepam & 271 & $243 \mathrm{BP}, 208,165$ \\
\hline 5 & \multicolumn{4}{|c|}{ Diazepam $=$ Base Peak } \\
\hline \multicolumn{4}{|c}{} \\
\hline
\end{tabular}

method searched for the seven analytes previously mentioned, including the internal standard, and was divided into five segments (Table 3). Identification was based on mass spectral and retention time matches.

\subsection{2 (c) Instrumentation: $H P L C / D A D$}

The samples were analyzed on a Perkin Elmer (PE) 200 series HPLC system, equipped with a PE 235 C diode-array detector, 200 series pump and a $101 \mathrm{LC}$ oven. The C-8 column was held constant at $30{ }^{\circ} \mathrm{C}$ and the detector had a fixed wavelength of $245 \mathrm{~nm}$. Mobile phase was comprised of a mixture of $16.5 \%$ acetonitrile to $57 \% \mathrm{pH} 6$, $0.1 \mathrm{M}$ sodium acetate buffer to $26.5 \%$ methanol and had a flow rate of $2.0 \mathrm{~mL} /$ minute. The total run time of the method was 20 minutes. 


\subsection{Basic Drugs: Quantitative Blood Analysis by GC/NPD}

The basic drug method was used to quantitate diphenhydramine, and methadone. Cerilliant Corporation (Round Rock, TX), stock standards were used to prepare working standards. The working standards were serially diluted to comprise a four point calibration curve ranging from $0.1 \mathrm{mg} / \mathrm{L}$ to $2.0 \mathrm{mg} / \mathrm{L}$. If necessary, samples were diluted to bring their concentrations within the established limits of the curve. Alltech Associates (State College, PA) stock standards were used to prepare a $0.5 \mathrm{mg} / \mathrm{L}$ working control. Linear regression was used to calculate sample concentrations.

Each $1 \mathrm{~mL}$ blood sample was fortified with $1 \mathrm{~mL}$ of mepivacaine internal standard $(0.5 \mathrm{mg} / \mathrm{L})$ and $1 \mathrm{~mL}$ of $\mathrm{pH} 9$ saturated sodium borate buffer. The samples were vortex-mixed, then $10 \mathrm{~mL}$ of n-butyl chloride was added. The mixture was capped and rotated for 15 minutes followed by centrifugation (3000 RPM) for 5 minutes. The organic fraction was then back extracted into $1.5 \mathrm{~mL}$ of $0.1 \mathrm{~N} \mathrm{HCl}$. The solution rotated for 15 minutes, followed by centrifugation (3000 RPM) for 5 minutes. The organic layer was aspirated to waste, after which, the aqueous layer was alkalinized with $2 \mathrm{~mL}$ of sodium carbonate/ sodium bicarbonate $(\mathrm{pH} 9.8)$ buffer and re-extracted into $10 \mathrm{~mL}$ of $\mathrm{n}$ butyl chloride. The organic layer was transferred into a clean tube and evaporated down to approximately $3 \mathrm{~mL}$ at $40{ }^{\circ} \mathrm{C}$ under a stream of nitrogen. A $100 \mu \mathrm{L}$ aliquot of concentrated $\mathrm{HCl}$ in methanol was added to each sample before drying to completion. The extracts were reconstituted with $50 \mu \mathrm{L}$ of ethanol and aliquoted into autosampler vials for analysis.

Hewlett-Packard's 5890 Series II GC model, equipped with an NPD and a 7673 autosampler was used for quantitative analysis. A DB-1 (100\% Methylpolysiloxane) 
megabore column $(15 \mathrm{~m}$ X $0.53 \mathrm{~mm}$ ID X $1.5 \mu \mathrm{m}$ film thickness, Agilent Technologies, Palo Alto, CA) was used for chromatographic separation. The initial oven temperature was $120{ }^{\circ} \mathrm{C}$ for 1 minute then ramped at $10{ }^{\circ} \mathrm{C} /$ minute to $290{ }^{\circ} \mathrm{C}$ and held at for one minute. Identification was based on relative retention times and previous GC/MS confirmation.

\subsection{Opiates}

Three opiate methods have been used by the MDME- Toxicology laboratory since 2006. Originally the opiate method was divided into two separate assays. Opiate I included morphine, codeine and 6-monoacetylmorphine (6-MAM). Opiate II method included hydrocodone, hydromorphone and oxycodone. As of January 2009, the opiate assay was combined into one comprehensive method to include all six of the previously mentioned opiates as well as the addition of oxymorphone. Both Opiate I and Opiate II methods were analyzed by GC/MS utilizing single ion monitoring (SIM), each with a linear range of $10-1,000 \mathrm{ng} / \mathrm{mL}$. The comprehensive method was analyzed by $\mathrm{GC} / \mathrm{MS} / \mathrm{MS}$ and had a linear range of $5-500 \mathrm{ng} / \mathrm{mL}$ for hydrocodone and $5-1,000 \mathrm{ng} / \mathrm{mL}$ for the remaining six opiate compounds.

\subsubsection{Preparation of Standards for Opiates by GC/MS (SIM)}

Stock standards, obtained from Cerilliant Corporation (Round Rock, TX), were used to prepare working standards. The working standards were serially diluted to comprise a four point calibration curve for the Opiate I method and a five point 
calibration curve for the Opiate II method. Both methods had a linear range of 10-1,000 $\mathrm{ng} / \mathrm{mL}$. After preparation, the standards were spiked in drug-free whole blood. Whenever necessary, samples that exceeded the limits of quantitation were diluted, to bring their concentrations within range, and reanalyzed on the appropriate instrument. Additional stock standards were purchased from Alltech Associates (State College, PA) to prepare controls. Both a high and a low working control $(50 \mathrm{ng} / \mathrm{mL}$ and $500 \mathrm{ng} / \mathrm{mL})$ were prepared for Opiate I, while a single $25 \mathrm{ng} / \mathrm{mL}$ working control was prepared for Opiate II. All standard and sample concentrations were calculated using linear regression.

\subsubsection{Extraction Procedure for Opiates by GC/MS (SIM)}

\subsection{2 (a) Extraction: Opiate I by GC/MS (SIM)}

To each $1 \mathrm{~mL}$ blood sample, $100 \mu \mathrm{L}$ of internal standard (morphine- D3, codeineD3 and 6-MAM - D3; final concentration equaled $50 \mathrm{ng} / \mathrm{mL}$ ) was added followed by $4 \mathrm{~mL}$ of $\mathrm{pH} 6,0.1 \mathrm{M}$ sodium phosphate buffer. The samples were then vortex-mixed, followed by a 15 minute equilibration. Afterwards, the samples were centrifuged (3500 RPM) for 10 minutes. The supernatant was transferred to a clean tube and submitted for automated solid-phase extraction preparation on Caliper Life Sciences (Hopkinton, PA) RapidTrace $^{\mathrm{TM}}$ system.

\subsection{2 (b) Extraction: Opiate II by GC/MS (SIM)}

To each $1 \mathrm{~mL}$ blood sample, $100 \mu \mathrm{L}$ of internal standard (hydrocodone- D3, hydromorphone- D3 and oxycodone - D3; final concentration equaled $25 \mathrm{ng} / \mathrm{mL}$ ) was 
added followed by $50 \mu \mathrm{L}$ of methoxamine (MOX) reagent. The samples were then vortex-mixed, followed by a 10 minute equilibration. Afterwards, the samples were heated at $56{ }^{\circ} \mathrm{C}$ for 15 minutes and allowed to cool. Then $4 \mathrm{~mL}$ of $0.1 \mathrm{M}, \mathrm{pH} 6$ sodium phosphate buffer was added. The samples were vortex-mixed and centrifuged at 3500 RPM for 10 minutes. The organic fraction was transferred to a clean tube and submitted for automated solid-phase extraction preparation on Caliper Life Sciences (Hopkinton, PA) RapidTrace ${ }^{\mathrm{TM}}$ system.

\subsection{2 (c) Automated Extraction: Opiates I and II by GC/MS (SIM)}

All solvents used for the rapid trace system were high purity grade. Clean Screen ${ }^{\mathrm{TM}}$ extraction columns (Worldwide Monitoring, Inc.) were conditioned with the elution solvent. Opiate I elution solvent consisted of methylene chloride: $\mathrm{IPA}: \mathrm{NH}_{4} \mathrm{OH}$; 80:20:2. Opiate II elution solvent was comprised of ethyl acetate: $\mathrm{NH}_{4} \mathrm{OH} ; 98: 2$. Afterwards, the columns were additionally conditioned with methanol, followed by water and finally pH 6 sodium phosphate buffer $(0.1 \mathrm{M})$. The blood samples were then loaded onto the columns ( $2 \mathrm{~mL} / \mathrm{min})$ and sequentially rinsed with water, $\mathrm{HCl}$ and methanol. The columns were dried with ultra zero grade air. Three milliliters of elution solvent was passed through the column at $1 \mathrm{~mL} / \mathrm{min}$ and the extract collected in a clean screw cap culture tube. The eluent was evaporated to dryness at $40{ }^{\circ} \mathrm{C}$ under a stream of nitrogen.

\subsubsection{Derivatization: Opiates I and II by GC/MS (SIM)}

Opiate I dried extracts were derivatized with $100 \mu \mathrm{L}$ of 1,1,1,3,3,3- Hexafluoro-2propanol (HFIP) followed by $100 \mu \mathrm{L}$ of pentafluoropropionic acid anhydride (PFAA). 
Opiate II extracts were derivatized using $100 \mu \mathrm{L}$ of acetic anhydride and $15 \mu \mathrm{L}$ of pyridine. The samples containing derivatizing reagent were then heated at $65{ }^{\circ} \mathrm{C}$ and $75^{\circ} \mathrm{C}$, for Opiate I and Opiate II, respectively. Afterwards, all samples were cooled to room temperature, then dried to completion at $40{ }^{\circ} \mathrm{C}$ under a stream of nitrogen. All extracts were reconstituted with $50 \mu \mathrm{L}$ of ethyl acetate and aliquoted into autosampler vials.

\subsubsection{Instrumentation: Opiates by GC/SIM-MS}

The Hewlett Packard 6890 series gas chromatograph coupled with a 5973 mass selective detector was utilized for quantitative analysis. A deactivated capillary guard column (5m X $0.250 \mathrm{~mm})$ was connected to a DB-5 (5\% phenyl methyl polysiloxane) analytical column $(15 \mathrm{~m}$ X $0.25 \mathrm{~mm}$ ID X $0.25 \mu \mathrm{m}$ film thickness; Agilent Technologies, Palo Alto, CA) to achieve chromatographic separation. The initial oven temperature was set for $65^{\circ} \mathrm{C}$ and held for 1 minute, then ramped $15{ }^{\circ} \mathrm{C} /$ minute to $300{ }^{\circ} \mathrm{C}$. The collection methods were divided into segments based on retention time of each analyte (Table 4). Identification was made on the basis of mass spectral matches of the appropriate ion ratios $( \pm 20 \%)$.

\subsubsection{Opiates: Quantitative Blood Analysis by GC/MS/MS}

This method was utilized to quantify hydrocodone, oxycodone, codeine, hydromorphone, morphine, 6-monoacetylmorphine (6-MAM) and oxymorphone. Stock standards obtained from Cerilliant Corporation (Round Rock, TX) were used to prepare working standards. The working standards were serially diluted to comprise a six point 
calibration curve ranging from $5 \mathrm{ng} / \mathrm{mL}$ to $1,000 \mathrm{ng} / \mathrm{mL}$. If necessary, samples were diluted to bring their concentrations within the established limits of the curve. Alltech Associates (State College, PA) stock standards were used to prepare two working controls, $50 \mathrm{ng} / \mathrm{mL}$ and $500 \mathrm{ng} / \mathrm{mL}$, respectively. Linear regression was used to calculate sample concentrations.

To each $1 \mathrm{~mL}$ blood sample, $100 \mu \mathrm{L}$ of deuterated internal standard mix (Table 5) and $4 \mathrm{~mL}$ of $0.1 \mathrm{M}, \mathrm{pH} 6$ sodium phosphate buffer were added. The samples were vortex-mixed and centrifuged at 3500 RPM for 10 minutes. The samples were then submitted for automated solid-phase extraction (SPE) on Caliper Life Sciences (Hopkinton, PA) RapidTrace ${ }^{\mathrm{TM}}$ system. All solvents used were high purity grade. Clean Screen ${ }^{\mathrm{TM}}$ extraction columns (Worldwide Monitoring, Inc.) were conditioned with the elution solvent (methylene chloride: IPA: $\mathrm{NH}_{4} \mathrm{OH} ; 80: 20: 2$ ), followed by methanol, then water and, finally, $\mathrm{pH} 6$ sodium phosphate buffer $(0.1 \mathrm{M})$. The blood samples were then loaded onto the columns $(2 \mathrm{~mL} / \mathrm{min})$ and sequentially rinsed with water, $\mathrm{HCl}$ and methanol. The columns were dried with ultra zero grade air. Three milliliters of elution solvent was passed through the column at $1 \mathrm{~mL} / \mathrm{min}$ and the extract collected in a clean screw cap culture tube. The eluent was evaporated to dryness at $40{ }^{\circ} \mathrm{C}$ under a stream of nitrogen. The dried extracts were derivatized with $100 \mu \mathrm{L}$ of propionic anhydride, catalyzed with $25 \mu \mathrm{L}$ of pyridine, and heated at $60{ }^{\circ} \mathrm{C}$ for 30 minutes. The samples were then dried to completion at $40{ }^{\circ} \mathrm{C}$ under a stream of nitrogen. The extracts were reconstituted with $50 \mu \mathrm{L}$ of ethyl acetate and aliquoted into autosampler vials. 
Table 4: Opiate Compounds Quantitated Using GC/ SIM- MS

\begin{tabular}{|c|c|c|c|c|c|c|}
\hline Method & Segment & Analytes & Molecular Ion & Quantifying Ion & Qual & Ions \\
\hline \multirow{6}{*}{ Opiate I } & \multirow{2}{*}{1} & Morphine & 577 & 414 & \multicolumn{2}{|c|}{430} \\
\hline & & Morphine- D3 $^{1}$ & - & 417 & \multicolumn{2}{|c|}{-} \\
\hline & \multirow{2}{*}{2} & Codeine & 446 & 282 & \multicolumn{2}{|c|}{445} \\
\hline & & Codeine $-\mathrm{D} 3^{1}$ & - & 285 & \multicolumn{2}{|c|}{-} \\
\hline & \multirow{2}{*}{3} & 6-MAM & 473 & 414 & \multicolumn{2}{|c|}{430} \\
\hline & & $6-\mathrm{MAM}-\mathrm{D} 3^{1}$ & - & 417 & \multicolumn{2}{|c|}{-} \\
\hline \multirow{6}{*}{ Opiate II } & \multirow{2}{*}{1} & Hydrocodone & 329 & 328 & \multicolumn{2}{|c|}{297} \\
\hline & & Hydrocodone $-\mathrm{D} 3^{1}$ & - & 331 & & \\
\hline & \multirow{2}{*}{2} & Oxycodone & 386 & 386 & 230 & 343 \\
\hline & & Oxycodone-D3 ${ }^{1}$ & - & 389 & \multicolumn{2}{|c|}{-} \\
\hline & \multirow{2}{*}{3} & Hydromorphone & 356 & 356 & 283 & 314 \\
\hline & & Hydromorphone -D3 ${ }^{1}$ & - & 359 & \multicolumn{2}{|c|}{ - } \\
\hline
\end{tabular}

${ }^{1}$ Only quantifying ions were collected for internal standards 
A Varian CP-3800 gas chromatograph coupled with a 1200L Triple Quadrupole Mass Spectrometer and CP-8400 autosampler system was utilized for quantitative analysis. A deactivated capillary guard column $(5 \mathrm{~m} \mathrm{X} 0.250 \mathrm{~mm})$ was connected to a DB-5 (5\% phenyl methyl polysiloxane) analytical column (15m X $0.25 \mathrm{~mm}$ ID X $0.25 \mu \mathrm{m}$ film thickness; Agilent Technologies, Palo Alto, CA). The initial oven temperature was set for $65{ }^{\circ} \mathrm{C}$ and held for 1 minute, then ramped $15{ }^{\circ} \mathrm{C} /$ minute to $315{ }^{\circ} \mathrm{C}$ (total run time $=17.67$ minutes). The collection method was divided into four segments based on retention time for each of the seven analytes and their respective deuterated internal standards (Table 5). Identification was made on the basis of mass spectral matches of the appropriate ion ratios $( \pm 30 \%)$.

Table 5: Opiate Compounds Quantitated Using GC/MS/MS

\begin{tabular}{|c|c|c|c|c|c|}
\hline \multirow{3}{*}{$\frac{\text { Segment }}{1}$} & \multirow{2}{*}{$\begin{array}{l}\text { Analytes } \\
\text { Hydrocodone }\end{array}$} & \multirow{2}{*}{$\begin{array}{c}\begin{array}{c}\text { Molecular } \\
\text { Ion }\end{array} \\
299\end{array}$} & \multirow{2}{*}{$\begin{array}{c}\begin{array}{c}\text { Quantifying } \\
\text { Ion }\end{array} \\
242 \mathrm{BP}\end{array}$} & \multicolumn{2}{|c|}{$\begin{array}{c}\text { Qualifying } \\
\text { Ions }\end{array}$} \\
\hline & & & & 270 & 299 \\
\hline & Hydrocodone - D3 ${ }^{1}$ & 302 & 245 & - & 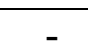 \\
\hline \multirow{4}{*}{2} & Codeine & 355 & 282BP & 298 & 355 \\
\hline & Codeine - D ${ }^{1}$ & 358 & 285 & 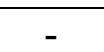 & 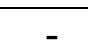 \\
\hline & Oxycodone & 371 & 314BP & 212 & 371 \\
\hline & 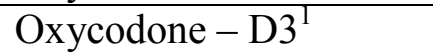 & 374 & 215 & & 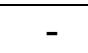 \\
\hline \multirow{4}{*}{3} & Hydromorphone & 341 & 285BP & 229 & 341 \\
\hline & Hydromorphone - D3 ${ }^{1}$ & 344 & 288 & - & - \\
\hline & 6-Acetylmorphine & 383 & 327BP & 204 & 383 \\
\hline & 6-Acetylmorphine - D $3^{1}$ & 386 & 207 & - & - \\
\hline \multirow{4}{*}{4} & Morphine & 397 & 341BP & 218 & 397 \\
\hline & Morphine - D3 ${ }^{1}$ & 400 & 221 & - & - \\
\hline & Oxymorphone & 413 & 357BP & 284 & 413 \\
\hline & 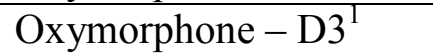 & 416 & 360 & - & - \\
\hline
\end{tabular}

${ }^{1}$ Qualifying ions were not collected for the internal standards 


\subsection{Microbial Study}

\subsubsection{Preparation of Nutrient Agar plates}

Five-hundred milliliters of double-distilled water was added to 11.5 grams of Becton, Dickinson and Company brand (Sparks, MD) nutrient agar. The mixture was heated until the agar went into solution, then autoclaved for 15 minutes at $121{ }^{\circ} \mathrm{C}$. After

which, the solution cooled to approximately $50{ }^{\circ} \mathrm{C}$ and was poured into $100 \times 15 \mathrm{~mm}$ petri dishes (Medegen Medical Products, Gallaway, TN). The agar plates were stored at room temperature $\left(25^{\circ} \mathrm{C}\right)$ for 24 hours, followed by refrigeration until use.

\subsubsection{Phosphate Buffered Saline Standards}

For the stability and microbial studies, drug standards made in phosphate buffered saline (PBS) solution were needed. Drug standards at the MDME- toxicology laboratory are often prepared in organic solvents (e.g. methanol or acetonitrile). However, for the purpose of these experiments, it was disadvantageous to use standards that introduced additional carbon sources into the matrices. As a result, powdered standards were mixed with 1X PBS solution and used as needed.

The procedure was conducted as follows: $25 \mathrm{~mL}$ volumetric flasks, individual $1 \mathrm{mg} / \mathrm{mL}$ stock standards of diazepam, codeine and hydrocodone were prepared. Twenty-five milligrams of hydrocodone bitartrate (Endo Laboratiories, Inc., Garden City, $\mathrm{NY}$ ), $24.9 \mathrm{mg}$ of codeine sulfate (Lilly, Inc., Indianapolis, IN) and $25.2 \mathrm{mg}$ of diazepam (La Roche, Inc, Nutley, NJ) were added to their respective volumetric flasks containing PBS solution. The solutions were vortex mixed. Codeine and hydrocodone stock 
standards were transferred into individual amber vials fitted with screw cap lids. The diazepam standard however, would not dissolve. Therefore, that mixture (in its flask) was sonicated for 45 minutes in a water bath. The standard remained a heterogeneous mixture. A review of the original container that held the powdered standard was only marked diazepam and did not give any additional information about its formulation. However, the diazepam powder was visibly different from that of the codeine and hydrocodone standards. The diazepam powder was tan in color and had a consistency similar to a starch-based powder (e.g. baby powder), as opposed to the white, salt based opiate powders used. Therefore, it was determined that the properties of the diazepam standard would not allow for the preparation of a homogenous solution. A subsequent review of the literature revealed that diazepam (along with other benzodiazepines) is not soluble in water (43), therefore explaining its resistance to the water based PBS solution. Consequently, diazepam could no longer be used for this portion of the study.

One hundred micro-liters of codeine and hydrocodone stocks were added to individual $10 \mathrm{~mL}$ volumetric flasks and mixed with $9,900 \mu \mathrm{L}$ of PBS solution to make $0.01 \mathrm{mg} / \mathrm{mL}(10,000 \mathrm{ng} / \mathrm{mL})$ working stock solutions. The solutions were then transferred into amber vials fitted with screw caps and stored under refrigeration $\left(2{ }^{\circ} \mathrm{C}\right)$.

\subsubsection{Aerobic and Anaerobic Microbial Experiments}

All of the postmortem blood used for this experiment was stored in tubes containing the anti-microbial agent sodium fluoride $(\mathrm{NaF})$. In an effort to test the effectiveness of the $\mathrm{NaF}$, blood samples needed to be inoculated in a nutrient-rich environment to determine if any microbial activity would exist. The PM blood used for 
this part of the experiment was collected from the chest of a decomposing cadaver. Based on the odor and discoloration of the body, it was evident that microbial activity was flourishing. The blood (green in color) was collected in $2 \mathrm{BD}$ vacutainer gray-top tubes $(10 \mathrm{~mL}$ each) and 1 red-top polypropylene tube $(50 \mathrm{~mL}$ total). The red-top contained $\mathrm{NaF}$ that was added by the MDME staff upon receipt of the tubes, while the gray-top was commercially prepared. Upon sample collection, all of the tubes were inverted multiple times to ensure proper mixing of the $\mathrm{NaF}$ with the blood.

Nutrient agar plates were inoculated in duplicate, each with $75 \mu \mathrm{L}$ of chest blood from the red-top and gray-top tubes. Additionally $75 \mu \mathrm{L}$ of antemortem blood, stored in a gray-top tube was also inoculated. Disposable sterile loops (Thermo Fisher Scientific, Waltham, MA) were used to streak the plates. For the aerobic experiment, a set of duplicate plates were stored in the dark for 11 days at $17{ }^{\circ} \mathrm{C}$. For the anaerobic experiment, an additional set of duplicate plates were stored in a vented $\mathrm{BBL}^{\circledR}$ anaerobic system jar (Becton Dickinson Microbiology Systems, Cockeysville, MD). In order to provide the anaerobic environment, BD GasPak ${ }^{\mathrm{TM}}$ EZ Anaerobe Gas Generating Pouch system with indicator (Becton, Dickinson and Company, Sparks, MD) was used. Once the plates were properly positioned in the jar, the foil wrapping was removed from the EZ gas pouch. The release of carbon monoxide (thereby providing an anaerobic environment) was activated when the pouch was open and exposed to air. Upon activation, the lid was sealed and the jar was stored in the dark for 11 days at $17^{\circ} \mathrm{C}$. 
4.5.4 Assessing the Usefulness of Codeine and Hydrocodone as a Food Source for Bacteria

Since a major part of this project was to determine the reason for drug concentration changes among antemortem and postmortem samples, it was imperative to evaluate the role of microbial activity. In an effort to determine if drug compounds could themselves be used as a food source for bacteria, the following procedure was employed.

A DH5a strain of Eschericia coli (E. coli) and a PAO1 strain of Pseudomonas aeruginosa (Pseudomonas) was plated onto LB agar plates and allowed to grow for 48 hours at $37{ }^{\circ} \mathrm{C}$. Individual colonies were transferred into two $50 \mathrm{~mL}$ polypropylene tubes (one for each bacteria) containing $8 \mathrm{~mL}$ of nutrient broth. The suspension was rocked for 24 hours, followed by 24 hour incubation $\left(37^{\circ} \mathrm{C}\right)$. The suspension was then centrifuged and the supernatant disposed. The cellular precipitate was rinsed with phosphate buffered saline (PBS) solution. That suspension was centrifuged and again the supernatant was disposed. This step was repeated twice. The cells were then resuspended in $5 \mathrm{~mL}$ of nutrient broth. A $1 \mathrm{~mL}$ aliquot of both suspensions was reserved for photometric reading.

A 96 well MT plate (9x12), manufactured by Biolog (Haywood, CA), was arranged as shown in Figure 5. Columns 3, 7, 11 and 12 were not used. Negative and positive controls were setup in the first two columns. One-hundred micro-liters of codeine and hydrocodone (prepared in 1X PBS solution) at concentrations of 100, 200 and $600 \mathrm{ng} / \mathrm{mL}$ each were aliquoted into columns 4-6 and 8-10. Afterwards, a baseline reading of the plate was taken using a Modulus Microplate reader (Turner Biosystems, Sunnyvale, CA) at a wavelength of $600 \mathrm{~nm}$. The plate was then refrigerated for 48 hours 
Figure 5: Arrangement of the 96 Well MT Plate

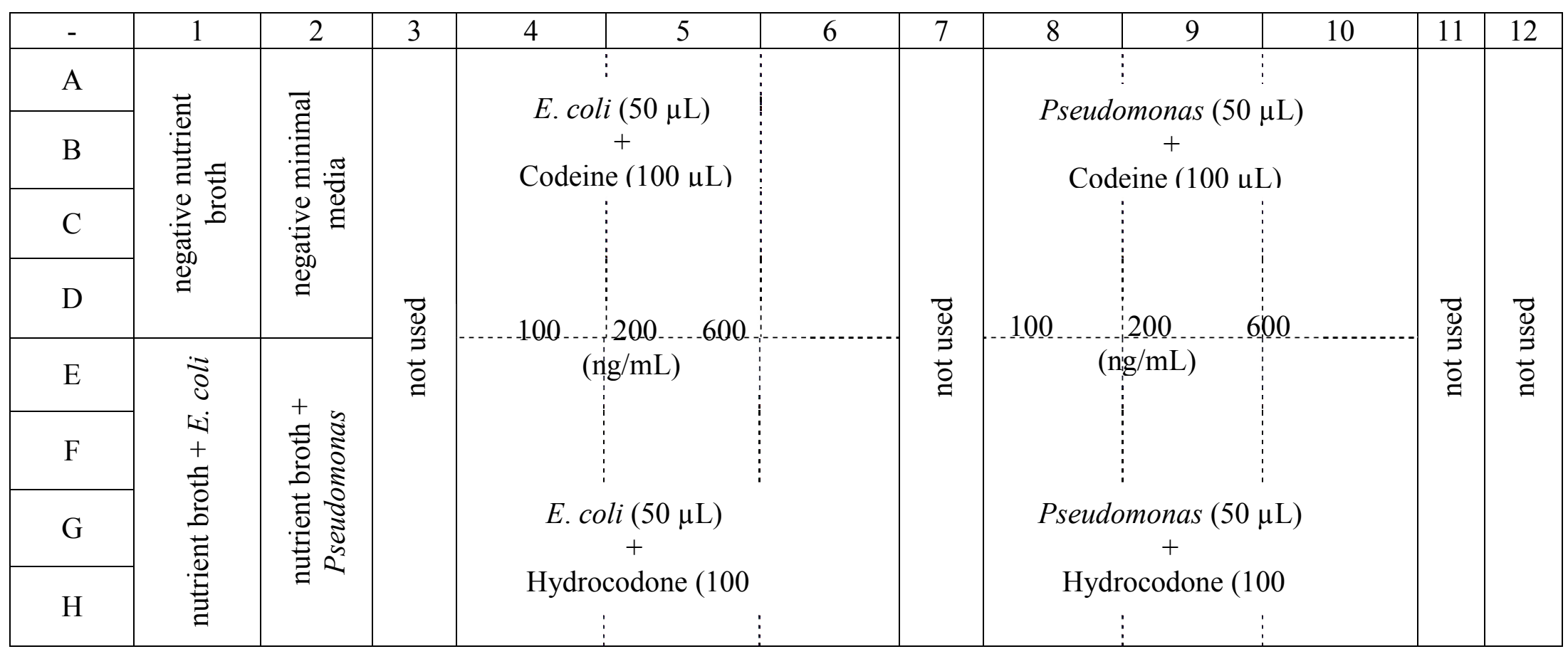


while the bacteria (E. coli and P. aeruginosa) were prepared. Upon which time, $50 \mu \mathrm{L}$ of E. coli was inoculated in columns 4-6, with $50 \mu \mathrm{L}$ Pseudomonas in columns 8-10. After which, another density reading was taken. Microplate readings continued daily for a total of 8 readings.

The $1 \mathrm{~mL}$ aliquot reserved for photometric reading was analyzed using a BioPhotometer (Eppendorf AG, Germany) at a $600 \mathrm{~nm}$ wavelength. This reading was taken to ensure the density of the bacteria population would be sufficient for use in this study.

\subsection{Drug Stability Study}

The purpose of this experiment was to assess the stability of hydrocodone and codeine in whole blood when stored in refrigeration $\left(2{ }^{\circ} \mathrm{C}\right)$ for three months. In the Peters article (44), four main types of drug stability were identified. They include long-term stability (examination of drug stability in storage), freeze/thaw stability (examines the effect of re-analysis of samples that have been frozen), in-process or bench top stability (assesses the stability of a drug during the sample preparation procedure) and finally processed sample stability, which addresses the stability of a drug while awaiting instrument injection. This experiment was solely focused on long-term stability. While some of the cases analyzed for this project were obtained a year or more before the inception of this research, many were analyzed within three months of the sample acquisition. The three month lapse typically accounted for the time required for routine laboratory analyses to be completed. At the completion of routine testing, research analysis could begin. An extended stability study $(3$ mon ths) was not conducted by the 
researcher as several articles $(21)(44)(45)(46)(47)$ have been published addressing the stability of drugs and drug classes used in this study (e.g. benzodiazepines, opioids and methadone).

One milliliter each of codeine and hydrocodone working stocks (prepared as described in section 4.5.2 Phosphate Buffered Saline Standards) were spiked into $48 \mathrm{~mL}$ of chest blood to give a final concentration of $200 \mathrm{ng} / \mathrm{mL}$ each. The mixture was rocked for 10 minutes to ensure equilibrium. Twenty milliliters of the spiked blood was separated into two gray-top vacutainer tubes (each tube held $10 \mathrm{~mL}$ ). The remaining $30 \mathrm{~mL}$ of spiked blood was stored in a red-top polypropylene tube (50 mL capacity). Stock, working stock and spiked blood tubes were kept in refrigeration $\left(2{ }^{\circ} \mathrm{C}\right)$ until further use. Additionally, in an effort to correlate drug stability with microbial activity, the chest blood spiked for this experiment was the same blood inoculated (without the drug spike) on the nutrient agar plates in section 4.5.3 Aerobic and Anaerobic Microbial Experiments. Similarly the hydrocodone and codeine standards were the same ones used in the MT plate experiment described in section 4.5.4.

\subsection{Calculations}

In order to better interpret the significance of the data, statistical analyses were conducted. Statistical testing was applied to the control data collected for each drug of interest. The controls, as opposed to the actual case blood samples, were analyzed because they had enough data points generated to produce relevant population sizes. Conversely, the cases used for this study had a finite amount of blood available for 
testing. Therefore, analyzing multiple replicates over a period of days was not feasible. The controls, however, were extracted and analyzed with every batch, thereby allowing for more replicates over a period of months to years depending on the assay.

As the testing for this project was conducted over a three year period, control lot numbers, and, at times, instrumentation were changed. The data that were produced for drugs analyzed on one instrument, but with multiple lot numbers, underwent one-way Analysis of Variance (ANOVA) testing, to test the hypothesis that the lot numbers were not different.

As an example, the diphenhydramine control $(0.50 \mathrm{mg} / \mathrm{L})$ was run forty-one times by GC/NPD and had three different lot numbers. The population sizes for lots 1,2 and 3 were 15,19 and 7, respectively. To determine if each lot produced similar results a oneway ANOVA with a 95 percent confidence interval, was calculated using Microsoft Excel's Data Analysis Toolpak software. The results given in Table 6 show both the summary and ANOVA analysis output. Listed within the summary is a breakdown of each lot number, indicating its population size and average (Table 6) control concentration. The ANOVA analysis results gave such parameters as the sum of squares (SS), degrees of freedom (df) and mean square (MS) values. Of particular interest were the calculated values for $F$ and $F$ crit. The $F$ value is the F-test (Equation 3 ) result that was calculated by the ANOVA. If $F>F_{\text {crit }}$, it was determined that a significant difference between the populations existed. The $F$ value for the diphenhydramine calculation was 2.839 , while $F_{c r i t}$ equaled 3.244. Therefore, in this example, $F$ was less than $F_{c r i t}$, indicating that the populations were not statistically different. As a result, the data produced by all 3 lot numbers were treated as one population. 
The control data for each drug was evaluated as described above. Once it was determined that the data could be combined, the population standard deviation (Equation 4) was calculated. After which, the coefficient of variation (CV), that is the percent relative error, was determined for each drug by using Equation 5. In keeping with the diphenhydramine example, the population mean and standard deviation values were 0.55 and 0.08 , respectively. Therefore the $\mathrm{CV}$, for this drug was approximately $15 \%$. The $\mathrm{CV}$ was used as a way to measure 'significance' among the intra-case data. In order to make a determination as to whether or not the concentration differences observed (within any given case) was statistically significant, the CV value was applied. If the differences in sample concentrations fell within the established $\mathrm{CV}$ (for example $\pm 15 \%$ ), then no significant difference was observed, however if the concentrations were not within the limits of the relative error as described by the $\mathrm{CV}$, then the concentrations were deemed statistically different.

Additionally, as methodology in the MDME - toxicology laboratory changed, so did the methodology for this project. As a result, some of the quantitative drug results were collected on two instruments. A two-way Analysis of Variance (ANOVA) with a $95 \%$ confidence interval was conducted to determine if the control values obtained on different instruments were statistically similar. If the F-test results generated by the ANOVA determined the control results were similar, then the data were treated as an entire population, regardless of instrumentation. If however, there was a significant difference between instruments, then Tukey's honestly significant difference (HSD) test (Equation 6) was employed on a post hoc basis. This was the case for clonazepam. 
Clonazepam data for this project were originally collected by GC/ECD. Eventually the method was modified and analyzed by LC/MS/MS. The F-test result (generated by the two-way ANOVA) equaled 26.84, while the $F_{\text {crit }}$ equaled 4.35. Since $F$ was greater than $F_{c r i t}$, the results indicated that the data produced on the two instruments differed. Therefore, the data were evaluated as two separate populations (one population per instrument), as opposed to being treated as a single population. In an attempt to check the validity of the ANOVA results, Tukey's HSD post hoc analysis was conducted.

Post hoc analysis is only used on data that has indicated a statistical difference. Tukey's HSD test compares the difference between mean values of sample populations to a newly calculated critical value (HSD ${ }_{\text {crit }}$ ). In the case of clonazepam, the control values generated by GC/ECD had a mean of $0.10 \mathrm{mg} / \mathrm{L}$ and the LC/MS mean control data equaled $0.13 \mathrm{mg} / \mathrm{L}$. The calculated mean difference between the two equaled 0.03 . When solving for $\mathrm{HSD}_{\text {crit, }}$ the critical value equaled 0.01. When the mean difference is greater than $\mathrm{HSD}_{\text {crit }}$ (as was the case with clonazepam) a significant difference exists. Consequently, there was a difference in the clonazepam data collected by GC/ECD when compared to that of the data collected by LC/MS/MS. As a result, the data were treated as two separate populations, with each having its own amount of relative error (CV).

Not only was it important to identify an acceptable amount of variance within an entire population, it was also imperative to determine which PM results were reasonable and which were not. Since each case had its own unique set of circumstances, it would not have been appropriate to implement a static scale in which to determine significance. Instead, dynamic calculations, which could be tailored to each case, were used. 
Equation 2: Average of $\boldsymbol{n}$ measurements ${ }^{(48)}$

$$
\operatorname{mean}(\bar{x})=\frac{\Sigma x_{i}}{n}
$$

$\Sigma x_{i}=$ sum of all the measurements

$\mathrm{n}=$ number of measurements

Equation 3: F-test for Comparison of Standard Deviations ${ }^{(48)}$

$$
F=\frac{s_{1}^{2}}{s_{2}^{2}}
$$

$\mathrm{s}=$ standard deviation

Equation 4: Standard deviation of $\boldsymbol{n}$ measurements ${ }^{(48)}$

$$
\begin{gathered}
s=\frac{\sqrt{\sum\left(x_{i}-\bar{x}\right)^{2}}}{(n-1)} \\
x_{i}=\text { sum of all measurements } \\
\bar{x}=\text { mean } \\
\mathrm{n}=\text { number of measurements } \\
\mathrm{n}-1=\text { degrees of freedom }
\end{gathered}
$$




\section{Table 6: Example of One-WAY ANOVA Data Output by Microsoft Exel ${ }^{\circledR}$ Generated when Comparing the Three Diphenhydramine Control Lots}

Anova: Single Factor

\begin{tabular}{ccccc}
\multicolumn{2}{c}{ SUMMARY } & & & \\
\hline Groups & Count & Sum & Average & Variance \\
\hline lot 1 & 15 & 8.624 & 0.574933 & 0.013845 \\
lot 2 & 19 & 10.5 & 0.552632 & 0.00242 \\
lot 3 & 7 & 3.46 & 0.494286 & 0.001762 \\
\hline
\end{tabular}

\begin{tabular}{cccccc} 
ANOVA & \multicolumn{7}{c}{$\begin{array}{c}\text { degrees } \\
\text { of } \\
\text { Source of } \\
\text { Variation }\end{array}$} & $\begin{array}{c}\text { Mean } \\
\text { Square }\end{array}$ & $F$ & F crit \\
\hline Between lots & 0.031157 & 2 & 0.015579 & 2.387292 & 3.244818 \\
Within lots & 0.247973 & 38 & 0.006526 & & \\
\hline
\end{tabular}

If $\mathrm{F}>\mathrm{F}$ crit: Significant difference exists If $\mathrm{F}<\mathrm{F}$ crit: no significant difference exists 


\section{Equation 5: Coefficient of Variation ${ }^{(48)}$}

$$
C V=\left(\frac{\text { population std dev. }}{\text { population mean }}\right) \times 100
$$

Equation 6: Tukey's Honestly Significant Difference Equation ${ }^{(70)}$

$$
H S D_{\text {crit }}=q \sqrt{\frac{M S_{\text {within }}}{n}}
$$

$\mathrm{HSD}_{\text {crit }}=$ newly calculated critical value $\mathrm{q}=$ reference value obtained from a studentized range table $\mathrm{MS}_{\text {within }}=$ Mean Square within value obtained from the ANOVA data output $\mathrm{n}=$ population size

*if mean difference $>\mathrm{HSD}_{\text {crit}}$, a significant difference exists 
Ratio calculations are common tools used in postmortem casework to determine if postmortem redistribution could have influenced the results. Whenever both postmortem and antemortem samples (for the same case) have been tested and rendered quantitative results a PM to AM ratio can be calculated. This comparison allows the interpreter to determine the true extent of the concentration disparity, as any time the ratio is 1.0 it is said that postmortem redistribution was likely a factor. Similarly, when comparing two postmortem sources (central and peripheral) if the resultant ratio $\unlhd$ sil.0, postmortem redistribution is believed to be the cause. When interpreting the results of both ratios, the higher the value is above 1.0, the more likely that redistribution has occurred. While both ratios are useful, only about 33\% of the cases submitted at the MDME Department are sent with AM specimens. In contrast, greater than $75 \%$ are submitted with both central and peripheral PM sources. Therefore, if a question about the occurrence of PMR arises, it is the central to peripheral that will most likely be available. For the purposes of this study, however, both central to peripheral as well as postmortem to antemortem calculations were applied whenever possible to better assess the intra-case relationships. Finally, it was imperative to determine what drug concentrations would be expected at the time of death. The pharmacodynamic process (section 1.4.2), which governs the body's ability to remove substances from its system, had to be taken into account when attempting to identify any concentration expectations. As indicated in Equation 7, when substituting the antemortem concentration for $N_{t}$, the antemortem to death (AMD) interval for $T$ and separately inputting the minimum and maximum elimination half-life values for $t_{1 / 2}$, an expected drug concentration range at the time of death could be 
calculated. The PM concentrations that fell within their expected ranges were believed to demonstrate drug metabolism (and elimination) occurring in a predicted fashion.

\section{Equation 7: Calculated Expected Drug Concentration Range at the Time of Death ${ }^{(22)}$}

$$
\ln N_{t}=\ln N_{0}-\ln 2\left(T / t_{1 / 2}\right)
$$

$$
\begin{gathered}
N_{t}=\text { Estimated concentration at the time of death } \\
N_{0}=\text { AM concentration of the sample } \\
\mathrm{T}=\text { Time between AM and death } \\
\mathrm{t}_{1 / 2}=\text { elimination half-life of the drug }
\end{gathered}
$$

The use of both the minimum and maximum elimination half-life values, resulted in a concentration range that could likely account for the majority of people (both fast and slow metabolizers). The range was critical in determining whether or not postmortem concentrations were significantly different than expected. The expected ranges vary by case and are addressed on a case-by-case basis in Chapter 6: Results and Discussion.

\subsection{Results and Discussion}

The data discussed in the following sections were from cases obtained by the Miami-Dade County Medical Examiner's Department. At the time of the sample testing for this project, the cases were no longer being actively analyzed in the toxicology laboratory. However, initial analyses were conducted by laboratory staffers to identify, 
and in most instances, quantify the drugs of interest, as part of the death investigative process. Any sample whose volume was not sufficient for repeat analyses by the researcher was still included in the study if a quantitative value was obtained as a part of routine laboratory analyses. Since it is of the utmost importance that the identities of the subjects remain private, each case has been assigned an alpha-numeric combination, not related to their actual case numbers.

\subsection{Opiates}

As discussed in section 1.7.2, opiates are drug class characterized by their analgesic properties. Users often establish a tolerance, which can make it difficult to interpret the true meaning of a given concentration determined in blood. The following sections will cover both prescription and illicit opiates. Through December 2008, the MDME- toxicology laboratory divided opiates into two analytical methods of analysis. The Opiate I method included morphine, codeine and 6- monoacetylmorphine (6-MAM), while the Opiate II method included hydrocodone, hydromorphone and oxycodone. Both methods were analyzed on an Agilent GC/MSD instrument (SIM-mode) and had a linear range of $10-1,000 \mathrm{ng} / \mathrm{mL}$. As of January 2009, the opiate methods were combined and analyzed by GC/MS/MS using Varian's 1200L system. The six previously mentioned analytes, plus the addition of oxymorphone were included in this comprehensive method. Hydrocodone had a linear range of $5-500 \mathrm{ng} / \mathrm{mL}$, all other opiates had a linear range of $5-1,000 \mathrm{ng} / \mathrm{mL}$ by GC/MS/MS. All three opiate methods were validated according to the procedures implemented by MDME- toxicology laboratory. 


\subsubsection{Heroin, Morphine and Codeine}

While, heroin, morphine and codeine are all separate drugs in their own right, they are being discussed collectively because they are often detected together. As described in Section 1.7.2, heroin synthesis also produces the byproduct acetylcodeine. As a result, when heroin is metabolized, codeine, 6-monoacetylmorphine (6-MAM) and morphine may also be produced. Additionally, even in the absence of heroin usage, morphine and codeine can still be detected together, as morphine is also the demethylated metabolite of codeine. Therefore, in cases where codeine has been identified, morphine is also commonly found. The cases investigated here have been divided into two sections. The first includes those morphine/ codeine (MOC) cases whose antemortem was collected within two elimination half-lives before death. Those cases are listed in Table 9 in order of increasing antemortem to death interval. Codeine has an elimination half-life ranging from $1.9-3.9$ hours with morphine ranging from $1.4-6.7$ hours and volumes of distribution of $3.5 \mathrm{~L} / \mathrm{kg}$ and $2-5 \mathrm{~L} / \mathrm{kg}$, respectively (50)(51). The second section covers the MOC cases whose AM was collected beyond two elimination halflives.

Because two instruments were used to obtain the data for this chapter, atwo-way ANOVA $(\alpha=0.05)$ was conducted to determine if there were any statistical differences between the two methods (Table 7). The ANOVA F-test values for morphine, codeine and 6-MAM were 48.8, 3.7 and 17.6, respectively. While the $\mathrm{F}_{\text {crit }}$ values were 6.0, 7.7 and 7.7 for morphine, codeine and 6-MAM, respectively. When the F-test value is greater than the $\mathrm{F}_{\text {crit, }}$ a significant difference exists. Therefore, analysis of variance concluded that the F-test values for both morphine and 6-MAM resulted in statistically 
different results between the two instruments, while codeine detection did not differ as a function of analytical methods. Subsequently, the morphine and 6-MAM intra-sampling data could not be evaluated as one population. Tukey's honestly significant difference (HSD) post hoc test was applied to both morphine and 6-MAM to determine how the data sets differed. The calculated critical values for Tukey's HSD were less than the mean differences calculated for morphine and 6-MAM (Table 7). When the mean difference $>$ $\mathrm{HSD}_{\text {crit, }}$ a significant difference does in fact exist. Therefore, the post hoc analysis supported the ANOVA results, which indicated that the populations were different. Consequently, the coefficient of variation for morphine and 6-MAM differed depending on the instrumentation used (Table 8). Conversely, since there was no significant difference among the codeine data, the populations (GC/SIM-MS and GC/MS/MS data) were combined and evaluated as one.

The morphine/ codeine (MOC) cases discussed were separated into two sections. The first being those cases whose antemortem blood was collected within two elimination half-lives before death (Table 9). The second section is comprised of the remaining MOC cases whose antemortem blood collection time exceeded two elimination half-lives (Table 10). All of the cases are presented in order of increasing antemortem to death (AMD) interval, as opposed to numerical order.

The antemortem sample for morphine/ codeine case 14 was collected 9 minutes before death. Analysis of the sample detected $48 \mathrm{ng} / \mathrm{mL}$ of morphine present. Aorta and iliac vein bloods were submitted for postmortem analyses. Aorta blood contained 339 $\mathrm{ng} / \mathrm{mL}$ of morphine, $18 \mathrm{ng} / \mathrm{mL}$ of codeine and $6.5 \mathrm{ng} / \mathrm{mL}$ of 6-MAM. Similarly, iliac vein blood was positive for morphine, codeine and 6-MAM as well, with concentrations 
Table 7: Two-Way Analysis of Variation Results for Morphine, Codeine and 6-MAM by Comparing Control Data for GC/SIM-MS to GC/MS/MS

\begin{tabular}{|ccccccccc|} 
Drug & Source of Variation & $\begin{array}{c}\text { Sum of } \\
\text { Squares }\end{array}$ & $\boldsymbol{d f}$ & $\begin{array}{c}\text { Mean } \\
\text { Square }\end{array}$ & $\begin{array}{c}\text { F-test } \\
\text { result }\end{array}$ & $\begin{array}{c}\text { F critical } \\
\text { value }\end{array}$ & $\begin{array}{c}\text { Tukey's } \\
\text { critical } \\
\text { value }\end{array}$ & $\begin{array}{c}\text { Mean } \\
\text { Difference }\end{array}$ \\
\hline Morphine & GC/MS vs. GC/MS & 666.80 & 5 & 666.80 & 48.80 & 5.99 & 4.27 & 14.14 \\
\hline Codeine & GC/MS vs. GC/MS & 70.66 & 3 & 70.66 & 3.69 & 7.71 & N/A & N/A \\
\hline 6-MAM & GC/MS vs. GC/MS & 240.97 & 3 & 240.97 & 17.60 & 7.71 & 5.21 & 13.50 \\
\hline
\end{tabular}

$>$ If $\mathrm{F}>\mathrm{F}$ critical, then results are significantly different

$>$ If $\mathrm{F}<\mathrm{F}$ critical, then results are similar

$>$ If mean difference $>$ Tukey's crit, then the results are significantly different

Table 8: Coefficient of Variation Results for Morphine, Codeine and 6-MAM

\begin{tabular}{|ccc|}
\hline Drug & Method & CV \\
\hline \multirow{2}{*}{ Morphine } & GC/SIM-MS & $10.0 \%$ \\
\cline { 2 - 3 } & GC/MS & $9.1 \%$ \\
\hline Codeine & GC/SIM-MS and GC/MS & $9.8 \%$ \\
\hline \multirow{2}{*}{ 6-MAM } & GC/SIM-MS & $8.8 \%$ \\
\cline { 2 - 3 } & GC/MS & $5.1 \%$ \\
\hline
\end{tabular}


of $139 \mathrm{ng} / \mathrm{mL}, 7 \mathrm{ng} / \mathrm{mL}$ and $5 \mathrm{ng} / \mathrm{mL}$, respectively. Statistical analyses determined that the PM aorta blood concentrations were different from the iliac vein blood concentrations. Additionally, both PM morphine concentrations were dis-similar to the antemortem concentration. Antemortem blood analysis was negative for codeine and 6MAM; however, urine screen results identified the presence of all three drugs. The detection of codeine and 6-MAM in the urine, but their absence in the AM blood suggested that during the time of AM blood collection the drugs were no longer being distributed. Instead they had been eliminated into liquid waste. The detection of both codeine and 6-MAM in the PM blood samples indicated storage of the drugs in tissues (at the time of the AM collection) and then their release upon death. During the 26 hour PMI, all three drugs were able to redistribute themselves. Aorta blood: AM, as well as ILBL:AM ratios equaled 6.8 and 2.8 for morphine and codeine, respectively. Additionally, C:P ratios equaled 2.4, 2.6 and 1.3 for morphine, codeine and 6-MAM, respectively. All the ratio data in this case were consistent with PMR.

At 23 minutes before death the AM blood for MOC 12 was collected. Upon analysis it was determined that $1,544 \mathrm{ng} / \mathrm{mL}$ morphine and $40 \mathrm{ng} / \mathrm{mL}$ of codeine were present. The presence of both morphine and codeine were the result of heroin usage. Heroin has an elimination half-life of 2-6 minutes (30). Because of this rapid metabolism, heroin is quickly eliminated from blood and metabolized into urine. Heroin has an elimination half-life of 2-6 minutes (30). Because of this rapid metabolism, heroin is quickly eliminated from blood and metabolized into urine. Heroin was detected only in the urine. However since urine is not a suitable sample for quantitative analyses (section 1.6.2) and heroin is rarely detected in blood, many laboratories (including 
Table 9: Morphine, Codeine and Heroin Antemortem samples collected within 2 elimination half-lives

Heroin $t_{1 / 2}=2-6$ minutes

$6-M A M t_{1 / 2}=2-25$ minutes

Morphine $t_{1 / 2}=1.4-6.7$ hours; $V_{d}=2-5 \mathrm{~L} / \mathrm{kg}$

Codeine $t_{1 / 2}=1.9-3.9$ hours; $V_{d}=3.5 \mathrm{~L} / \mathrm{kg}$

\begin{tabular}{|c|c|c|c|c|c|c|c|c|c|c|}
\hline $\begin{array}{l}\text { AM- } \\
\text { Death }\end{array}$ & Case \# & $\begin{array}{c}\text { Source } \\
\text { (whole blood) }\end{array}$ & $\begin{array}{c}\text { Concentration } \\
(\mathrm{ng} / \mathrm{mL})\end{array}$ & $\begin{array}{c}\text { AM } \\
\text { concentration } \\
(\mathrm{ng} / \mathrm{mL})\end{array}$ & Analyte & PM:AM & $C: P$ & $\begin{array}{c}\text { PMI } \\
\text { (hours) }\end{array}$ & Race/Sex & Age \\
\hline \multirow{2}{*}{$9 \min$} & \multirow{2}{*}{$\begin{array}{l}\mathrm{MOC} \\
14^{2}\end{array}$} & Aorta & $\begin{array}{c}\text { morphine } 339 \\
\text { codeine } 18 \\
\text { 6MAM } 6.5\end{array}$ & \multirow{2}{*}{ morphine 48} & $\begin{array}{c}\mathrm{MO} \\
\mathrm{CO} \\
6 \mathrm{MAM}\end{array}$ & $\begin{array}{c}7.1 \\
- \\
-\end{array}$ & \multirow{2}{*}{$\begin{array}{l}2.4 \\
2.6 \\
1.3\end{array}$} & \multirow[b]{2}{*}{26.5} & \multirow[b]{2}{*}{ WM } & \multirow[b]{2}{*}{49} \\
\hline & & Iliac & $\begin{array}{c}\text { morphine } 139 \\
\text { codeine } 7 \\
\text { 6MAM } 5 \\
\end{array}$ & & $\begin{array}{c}\text { MO } \\
\text { CO } \\
6 \mathrm{MAM} \\
\end{array}$ & $\begin{array}{c}2.9 \\
- \\
- \\
\end{array}$ & & & & \\
\hline \multirow{2}{*}{$23 \mathrm{~min}$} & \multirow{2}{*}{$\begin{array}{l}\mathrm{MOC} \\
12^{2}\end{array}$} & Mixed & $\begin{array}{c}\text { morphine } 2,945 \\
\text { codeine } 76 \\
\text { 6MAM } 858\end{array}$ & \multirow{2}{*}{$\begin{array}{c}\text { morphine } 1,544 \\
\text { codeine } 40 \\
\text { 6-MAM } 90\end{array}$} & $\begin{array}{c}\text { MO } \\
\text { CO } \\
6 \mathrm{MAM}\end{array}$ & $\begin{array}{l}1.9 \\
1.9 \\
9.5\end{array}$ & \multirow{2}{*}{$\mathrm{N} / \mathrm{A}$} & \multirow{2}{*}{18} & \multirow{2}{*}{ WM } & \multirow{2}{*}{49} \\
\hline & & IVC & $\begin{array}{c}\text { morphine } 2,539 \\
\text { codeine } 83 \\
\text { 6MAM } 1,255 \\
\end{array}$ & & $\begin{array}{c}\mathrm{MO} \\
\mathrm{CO} \\
6 \mathrm{MAM} \\
\end{array}$ & $\begin{array}{c}1.6 \\
2.1 \\
13.9 \\
\end{array}$ & & & & \\
\hline $45 \mathrm{~min}$ & $\begin{array}{c}\mathrm{MOC} \\
13^{2}\end{array}$ & CHBL* & $\begin{array}{c}\text { morphine } 259 \\
\text { codeine } 9.75\end{array}$ & $\begin{array}{c}\text { morphine } 104 \\
\text { codeine } 20\end{array}$ & $\begin{array}{l}\mathrm{MO} \\
\mathrm{CO}\end{array}$ & $\begin{array}{c}2.5 \\
0.49\end{array}$ & $\mathrm{~N} / \mathrm{A}$ & 48 & $\mathrm{BM}$ & 40 \\
\hline 1 hour & $\begin{array}{c}\text { MOC } \\
05^{1}\end{array}$ & Aorta & $\begin{array}{c}\text { morphine } 48 \\
\text { codeine } 25\end{array}$ & morphine 65 & MO & 0.7 & $\mathrm{~N} / \mathrm{A}$ & 13.5 & WM & 57 \\
\hline \multirow{2}{*}{2 hours } & \multirow{2}{*}{$\begin{array}{c}\text { MOC } \\
03^{1}\end{array}$} & Aorta & $\begin{array}{c}\text { morphine } 52 \\
\text { codeine } 698\end{array}$ & \multirow{2}{*}{$\begin{array}{c}\text { morphine } 17 \\
\text { codeine } 65\end{array}$} & $\begin{array}{l}\mathrm{MO} \\
\mathrm{CO}\end{array}$ & $\begin{array}{c}3.0 \\
10.7\end{array}$ & \multirow{2}{*}{$\mathrm{N} / \mathrm{A}$} & \multirow{2}{*}{47} & \multirow{2}{*}{$\mathrm{BF}$} & \multirow{2}{*}{31} \\
\hline & & Chest & $\begin{array}{c}\text { morphine } 71 \\
\text { codeine } 1,397\end{array}$ & & $\begin{array}{l}\mathrm{MO} \\
\mathrm{CO}\end{array}$ & $\begin{array}{c}4.2 \\
21.5\end{array}$ & & & & \\
\hline
\end{tabular}




\begin{tabular}{|c|c|c|c|c|c|c|c|c|c|c|}
\hline $\begin{array}{l}\text { AM- } \\
\text { Death }\end{array}$ & Case \# & $\begin{array}{c}\text { Source } \\
\text { (whole blood) }\end{array}$ & $\begin{array}{c}\text { Concentration } \\
(\mathbf{n g} / \mathbf{m L})\end{array}$ & $\begin{array}{c}\text { AM } \\
\text { concentration } \\
(\mathrm{ng} / \mathrm{mL})\end{array}$ & Analyte & PM:AM & $C: P$ & $\begin{array}{c}\text { PMI } \\
\text { (hours) }\end{array}$ & Race/Sex & Age \\
\hline \multirow[b]{2}{*}{2 hours } & \multirow{2}{*}{$\begin{array}{c}\mathrm{MOC} \\
08^{1}\end{array}$} & HTBL & $\begin{array}{c}\text { morphine } 105 \\
\text { codeine } 10\end{array}$ & \multirow[b]{2}{*}{ morphine 205} & $\begin{array}{l}\mathrm{MO} \\
\mathrm{CO}\end{array}$ & $\begin{array}{c}0.5 \\
- \\
\end{array}$ & 1.4 & \multirow[b]{2}{*}{23} & \multirow[b]{2}{*}{$\mathrm{BM}$} & \multirow[b]{2}{*}{37} \\
\hline & & ILBL & $\begin{array}{c}\text { morphine } 76 \\
\text { codeine } 10\end{array}$ & & $\begin{array}{l}\mathrm{MO} \\
\mathrm{CO}\end{array}$ & $\begin{array}{c}0.4 \\
- \\
\end{array}$ & 1.0 & & & \\
\hline 5 hours & $\begin{array}{c}\mathrm{MOC} \\
02^{1}\end{array}$ & Mixed* & morphine 30 & $\begin{array}{c}\text { morphine }<\mathrm{LOQ} \\
\text { codeine } 25\end{array}$ & $\mathrm{MO}$ & $\mathrm{N} / \mathrm{A}$ & $\mathrm{N} / \mathrm{A}$ & 19 & WF & 81 \\
\hline
\end{tabular}

${ }^{1} \mathrm{GC} / \mathrm{MS}$ linear range: $10-1,000 \mathrm{ng} / \mathrm{mL}$

${ }^{2} \mathrm{GC} / \mathrm{MS}^{2}$ linear range: $5-1,000 \mathrm{ng} / \mathrm{mL}$

*Average concentration

Morphine $=\mathrm{MO}$

Codeine $=\mathrm{CO}$

6-Monoacetylmorphone $=6-\mathrm{MAM}$

Table 9, continued 
MDME-toxicology lab) do not have a blood quantitative method for heroin. Instead 6monoacetlymorphine $\left(\mathrm{t}_{1 / 2}=6-25\right.$ minutes), the major metabolite of heroin, is more often encountered and quantitated, as it is more likely to be identified in whole blood (section $1.6 .3)$.

Postmortem mixed blood (MXBL) and inferior vena cava blood (IVC BL) were submitted for analysis following autopsy. Mixed blood had a morphine concentration of $2,945 \mathrm{ng} / \mathrm{mL}$ with a codeine concentration of $76 \mathrm{ng} / \mathrm{mL}$. Inferior vena cava blood had concentrations of $2,539 \mathrm{ng} / \mathrm{mL}$ and $83 \mathrm{ng} / \mathrm{mL}$ for morphine and codeine, respectively. All samples (PM and AM) were diluted to bring their concentrations within the linear range $(\mathrm{ULOQ}=1,000 \mathrm{ng} / \mathrm{mL})$ of the assay. The $\mathrm{PM}$ results for morphine were determined to be statistically similar to each other. This was also true for the PM results of codeine. However, both morphine and codeine results were determined to be statistically different from the AM concentrations (morphine $\mathrm{CV} \pm 9.1 \%$, codeine \pm 9.8\%). On the basis of the AM results the expected concentration range for morphine was $1,277-1,484 \mathrm{ng} / \mathrm{mL}$. Codeine had an expected range of $34.8-37.4 \mathrm{ng} / \mathrm{mL}$. The PM results for both drugs exceeded their linear range. The MXBL:AM ratio equaled 2.0 for both morphine and codeine, while IVC BL:AM ratio equaled 1.7 and 2.0 for morphine and codeine, respectively. When comparing the PM to AM data, one could conclude that PMR occurred during the 18 hour PMI. While PMR may have contributed to the elevated postmortem concentrations, it is more likely that the drug was still being absorbed at the time of death. The oversaturation of the body's system, due to an overdose of heroin, can significantly hinder the pharmacokinetic process. 
Morphine/codeine case 13 had AM blood drawn 45 minutes before death. Analysis of this sample detected the presence of both morphine and codeine at concentrations of $104 \mathrm{ng} / \mathrm{mL}$ and $20 \mathrm{ng} / \mathrm{mL}$, respectively. Chest blood (CHBL) samples in both red-top (RT) and gray-top (GT) tubes were submitted for postmortem analyses. The red-top chest blood sample had $228 \mathrm{ng} / \mathrm{ml}$ of morphine and $8.5 \mathrm{ng} / \mathrm{mL}$ of codeine. Gray-top chest blood had $290 \mathrm{ng} / \mathrm{mL}$ of morphine and $11 \mathrm{ng} / \mathrm{ml}$ of codeine. Since the PM samples were from the same source, their drug concentrations were averaged to give $259 \mathrm{ng} / \mathrm{mL}$ and $9.75 \mathrm{ng} / \mathrm{mL}$ for morphine and codeine, respectively. Chest blood to AM ratios for morphine and codeine equaled 2.6 and 0.49 , respectively. The ratio values suggested that PMR occurred for morphine, but not for codeine. The expected concentration ranges were in agreement with the ratio data, as PM morphine concentrations exceeded its expected range $(71.7-96.2 \mathrm{ng} / \mathrm{mL})$, while codeine was below its range of $15.2-17.5 \mathrm{ng} / \mathrm{mL}$. The decrease in codeine concentration from AM to PM sample, may appear to be due to metabolism. However, the 45 minute antemortem to death (AMD) interval would not have been enough time for that amount of metabolism. Conversely, the PMI lasted 48 hours. In that amount of time, anaerobic conversion could be a possibility. This could account for the decrease of codeine's concentration and the increase of morphine, as morphine is the demethylated metabolite of codeine. So while PMR may have caused the concentration changes, anaerobic activity cannot be ruled out. In which case, the process of chemical conversion was ceased once the samples were aliquoted into RT and GT tubes which contain the antimicrobial agent sodium fluoride. 
The antemortem blood for MOC 05 was collected within one hour of death and had a postmortem interval of 13.5 hours. The AM blood had a morphine concentration of $65 \mathrm{ng} / \mathrm{mL}$ and was negative for codeine. Postmortem aorta blood was collected at autopsy and submitted for testing. Quantitative analysis determined that morphine was present at a concentration of $48 \mathrm{ng} / \mathrm{mL}$ and codeine at $25 \mathrm{ng} / \mathrm{mL}$. The concentration differences for $\mathrm{AM}$ and $\mathrm{PM}$ morphine values were statistically different $(\mathrm{CV} \pm 10 \%)$. The PM:AM ratio of morphine was 0.8, which indicated a lack of PMR. Based on the data, it would appear as though before the collection of the AM sample, the body metabolized codeine to the extent to which it was undetectable. The presence of codeine in the PM does seem to indicate the sequestering of codeine in or around the heart tissue and released upon death. This equates to PMR of codeine, but not morphine. Morphine had an expected concentration range of $36.6-54.1 \mathrm{ng} / \mathrm{mL}$. The AOBL concentration fell within this range, reiterating that PMR did not occur for morphine.

Antemortem blood for MOC 03 was collected 2 hours before death. The AM results determined that morphine and codeine were present at concentrations of $17 \mathrm{ng} / \mathrm{mL}$ and $65 \mathrm{ng} / \mathrm{mL}$, respectively. The decedent in this case was prescribed Tylenol $3^{\circledR}$, a mixture of codeine and acetaminophen. Therefore, the presence of morphine in the AM was likely the result of drug metabolism. The expected calculated concentration range for morphine was $7.4-16.3 \mathrm{ng} / \mathrm{mL}$, while the range for codeine was $28.9-42.0 \mathrm{ng} / \mathrm{mL}$. Postmortem aorta (AOBL) and chest (CHBL) blood samples were also submitted for analysis $(\mathrm{PMI}=47$ hours $)$. Quantitative analysis of the aorta blood had a morphine concentration of $52 \mathrm{ng} / \mathrm{ml}$ and codeine of $698 \mathrm{ng} / \mathrm{mL}$. The chest blood had concentrations of $71 \mathrm{ng} / \mathrm{mL}$ and 1,397 $\mathrm{ng} / \mathrm{mL}$ for morphine and codeine, respectively. 
The PM results were not only statistically different from the antemortem concentration, but they were also different from each other. Additionally, the actual postmortem concentrations for both morphine and codeine well exceeded their expected concentration ranges. The AOBL:AM ratios equaled 2.6 and 11.6 for morphine and codeine, respectively. The CHBL:AM ratio for morphine was calculated at 3.55, while codeine was 23.3. Both of the PM:AM ratios indicated PMR of morphine and codeine.

In MOC 03, both AOBL and CHBL samples were collected from the central region of the body. However, the data indicated a more extensive redistribution present in the chest blood. The term 'chest blood' is a generic designation for pooled blood collected from inside the chest cavity. Therefore, a chest blood sample is often a mixture of blood that has been released from the lungs, liver, stomach and/ or various chambers of the heart. Further review of the medical records determined that the decedent vomited prior to the arrival of the ambulance. Therefore, it is possible that some of the stomach contents were aspirated into the lungs, thereby contaminating the chest blood sample. This in turn could account for chest blood codeine concentrations that were significantly higher than both the aorta and antemortem bloods (50\% and $96 \%$, respectively).

Morphine/ codeine (MOC) 08 was submitted with AM blood that was collected 2 hours before death. The AM sample had $205 \mathrm{ng} / \mathrm{mL}$ of morphine but no codeine detected. Upon autopsy, both postmortem heart and iliac vein bloods were submitted. Heart blood (HTBL) had a morphine concentration of $105 \mathrm{ng} / \mathrm{mL}$ and codeine of 10ng/mL. Iliac vein blood (ILBL) analyses determined concentrations of $76 \mathrm{ng} / \mathrm{mL}$ and $10 \mathrm{ng} / \mathrm{mL}$ of morphine and codeine, respectively. While the PM codeine concentrations were equal, the PM morphine concentrations were statistically different. Additionally, 
both PM morphine concentrations were also different from the AM concentrations. The central to peripheral ratios equaled 1.4 and 1.0 for morphine and codeine, respectively. The HTBL:AM ratio of morphine equaled 0.5, while the ILBL:AM morphine ratio equaled 0.4 .

For MOC 08, the central to peripheral ratios indicated that PMR was present for both morphine and codeine. However, the PM:AM ratios did not support this finding for morphine. A codeine ratio could not be calculated, as codeine was not detected in the AM sample. Therefore, upon examining the data, the researcher determined that PMR of codeine was present. The lack of codeine in the AM, but its presence in both PM samples indicated that codeine had both metabolized (to the point of non-detection in the AM) and sequestered itself during life. The 23 hour PMI was enough time to allow codeine to be released in both the central and peripheral regions of the body, thereby facilitating its PM detection. However, the same conclusion could not be drawn for morphine.

Morphine's elimination half-life varies from 1.3 to 6.7 hours. Based on this, the expected PM morphine concentrations would have been between $76.2-166.7 \mathrm{ng} / \mathrm{mL}$. Both HTBL and ILBL concentrations fell within this range, suggesting that metabolism occurred as expected. Therefore, based on this information it would appear as though PMR was not present. However, the value calculated for the central to peripheral blood ratio did exceed 1.0, thereby suggesting the presence of PMR. In a situation when calculated values used to indicate PMR disagree, it can be difficult to determine if it actually occurred or not. However, in this case, the PM concentrations were within their 
expected concentration range; therefore, the researcher determined that PMR was not a factor in this case.

Antemortem blood for MOC 02 was collected 5 hours before death. Antemortem codeine concentration equaled $25 \mathrm{ng} / \mathrm{mL}$, while morphine was detected below the LOQ (10 ng/mL by Agilent 5890 GC/MS system). Postmortem mixed blood samples (PMI = 19 hours) were submitted for analysis in both red-top and gray-top tubes. Both tubes were analyzed (on the same day as the antemortem) and both detected $30 \mathrm{ng} / \mathrm{mL}$ of morphine. Codeine was not detected in either PM tube. Since the PM results were identical and of the same source, the results were combined into one averaged value. The elimination half-life for codeine ranges between 1.9 - 3.9 hours. As a result, the expected codeine concentration after five hours of metabolism would have between 4.0$10.3 \mathrm{ng} / \mathrm{mL}$. The upper limit of the expected range would have just been equal to the LOD and the LOQ for the GC-SIM/MS method. When interpreting the PM data, it appears as though codeine was extensively metabolized into morphine during the AMD interval. This resulted in both the lack of codeine detection and the increase of morphine in the PM sample (relative to the AMBL sample).

Those cases whose antemortem samples were collected within one elimination half-life illustrated many different considerations when trying to interpret toxicological data. For example, when AM blood is collected more than a few minutes before death, drug metabolism can change both the content of the drugs detected as well as their concentrations. This was illustrated in MOC 02 when the AM sample (collected 5 hours before death) had a quantifiable amount of codeine. However, PM analysis was negative for codeine and had increased levels of morphine. Similarly, during the metabolic 
process some drugs sequester themselves in organs. In these situations, AM blood results can be negative, but PM analysis can give a positive result. Morphine/ codeine case 05 and 08 were examples of this. In both cases, only morphine was present in the antemortem samples. However, upon PM analysis, both morphine and codeine were detected in quantifiable amounts. Additionally, in both of these cases, the PM morphine concentrations fell within their expected concentration ranges, indicating a lack of PMR. This illustrates that, while under the same conditions two drugs can respond differently. While codeine was sequestered and released after death, morphine was eliminated at a predictable rate.

In MOC 03, both morphine and codeine were quantified in the AM blood. Postmortem submission included aorta and chest blood samples. Both PM samples contained morphine with concentrations at least three times greater than that of the AM, as well as codeine, which had 10 times greater than the AM sample. Since two hours elapsed between AM collection and death, it would be expected that the concentrations of morphine and codeine would have decreased. Instead the increased amount of drugs led to PM:AM ratios of 3.0 and 10.7 for morphine and codeine, respectively in the aorta blood. While, the chest blood sample had PM:AM ratios of 4.2 and 21.5 for morphine and codeine, respectively. Chest blood concentrations of morphine and codeine exceeded those of the other two specimens (morphine by $>25 \%$ and codeine by $>98 \%$ ). This was probably due to the contamination present in a pooled blood sample taken from the chest cavity. Such a sample is predisposed to redistribution of drugs being released from any of the organs present within the chest cavity. In this case, there was a 47 hour PMI which would be ample time for the process of PM release and redistribution to occur. 
In cases where it has been determined that postmortem redistribution was a factor, two important considerations are needed. First, the length of the PMI should be considered. Secondly, identification of the major source of contamination that led to the redistribution. Morphine/ codeine 03 was a case where PMR was present in both the aorta and chest blood samples. The collection of the PM samples was 47 hours after death. During this time, the codeine concentration from the chest blood sample was almost twice that of the aorta blood. The significantly increased amount of codeine in the chest may suggest that the main source of contamination for this sample came from the stomach. The stomach holds un-metabolized drugs and is anatomically positioned within close proximity to the chest cavity. Additionally, the concentration of codeine in the aorta blood was more than 11 times greater than was detected in the AM blood sample. While the aorta blood also had a significant amount of PMR, it is not located close enough to the stomach to have been contaminated by it directly. Instead, the aorta was likely contaminated by the lungs, as well as the pooled chest blood. Based on the data, the chest blood had a more significant contamination than the lungs.

Finally, testing more than one sample matrix can give additional information to aid in the interpretive process. In the case of MOC 12, for example, blood as well as urine samples were tested. While urine is only used for qualitative purposes, it was the only sample in which heroin was detected. Although the extremely high morphine concentration would be indicative of heroin usage, actually identifying heroin in the urine eliminated the need to infer its role in this case. Morphine/ codeine case 12 is a good example of how gathering as much data as possible (toxicological, as well as social and medical history) can aid in the interpretative process. 
The following cases had antemortem blood collected greater than or equal to 2 elimination half-lives before death (Table 10). While their AM sample collection times are not the most ideal, they still offer quantitative information of value. The AM blood for MOC 09 was collected 6.5 hours before death. The sample had a codeine concentration of $48 \mathrm{ng} / \mathrm{mL}$, however morphine was not detected. Postmortem aorta and iliac vein bloods were also submitted for analyses. Codeine concentrations were determined to be $34 \mathrm{ng} / \mathrm{mL}$ and $27 \mathrm{ng} / \mathrm{mL}$ for aorta blood and iliac vein blood, respectively. Statistical analyses determined that the $\mathrm{CV}$ range for codeine in the AOBL was $30.6-37.4 \mathrm{ng} / \mathrm{mL}$. The range for the ILBL sample was between $24.3-29.7 \mathrm{ng} / \mathrm{mL}$. Therefore, although the range values were close, ultimately the AOBL and ILBL concentrations are dis-similar to each other. Additionally, as with the AM sample neither postmortem sample detected the presence of morphine. The C:P ratio for codeine equaled 1.2, suggesting PMR. However, both PM:AM ratios were less than 1.0 (AOBL:AM $=0.68 ;$ ILBL:AM $=0.54)$, which would indicate a lack of PMR. The expected concentration range of codeine would have been between $4.5-15.1 \mathrm{ng} / \mathrm{mL}$. The concentrations of both PM samples exceeded this range. Therefore, it can be determined that while the drug continued to metabolize and decrease in concentration during the 6.5 hours between AM collection and death, during the 33.5 hour PMI drug redistribution did occur. This, thereby, resulted in elevated PM sample concentrations.

Morphine/ codeine case 11 had antemortem concentrations of $92 \mathrm{ng} / \mathrm{mL}$ and $17 \mathrm{ng} / \mathrm{mL}$ for morphine and codeine, respectively. The AM sample was collected 10 hours prior to death. As a result, the expected PM morphine concentrations would range between $0.65-32.7 \mathrm{ng} / \mathrm{mL}$, with codeine ranging from $0.4-2.9 \mathrm{ng} / \mathrm{mL}$. Mixed heart as 
well as inferior vena cava bloods was submitted for PM analyses. Nine and a half nanograms per milliliter of morphine were detected in the mixed heart sample, while $8 \mathrm{ng} / \mathrm{mL}$ was detected in the IVC blood. These concentrations were determined to be statistically similar. Codeine was not detected in either PM sample. The lack of codeine was consistent with the expected concentration range, as the values obtained there were below both the LOD (3 ng/mL) and LOQ ( $5 \mathrm{ng} / \mathrm{mL})$ of the GC/MS/MS analysis. However, the morphine concentration detected fell within its expected range. Therefore, there is no reason to believe that PMR affected this case.

The antemortem blood submitted for MOC 06 was negative for morphine and codeine. However, the antemortem urine sample was positive for morphine. As a result, the PM blood was analyzed by the researcher to determine if morphine or codeine could be detected. The postmortem blood submission consisted of both aorta and iliac vein blood samples. Morphine concentrations of $17 \mathrm{ng} / \mathrm{mL}$ for both AOBL and ILBL samples were detected. Codeine had concentrations of $25 \mathrm{ng} / \mathrm{mL}$ and $27 \mathrm{ng} / \mathrm{mL}$ in aorta and iliac vein bloods, respectively. These samples were analyzed by SIM GC/MS and had a LOD/ LOQ of $10 \mathrm{ng} / \mathrm{mL}$. Therefore, if morphine and codeine were present in the AM sample, their concentrations would have been below $10 \mathrm{ng} / \mathrm{mL}$. As a result, the detection of morphine and codeine in both PM samples was indicative of increased concentrations for both drugs. This would suggest that during the 48 hour PMI, PMR occurred.

Morphine/ codeine case 15 had an AM blood sample collected 12 hours before death. The AMBL had a morphine concentration of $98 \mathrm{ng} / \mathrm{mL}$. Based on this information the expected morphine concentration would be between $0.3-28.9 \mathrm{ng} / \mathrm{mL}$. Heart blood was submitted for PM analysis in both red-top and gray-top tubes. Red-top 
Table 10: Morphine, Codeine and Heroin Antemortem samples collected after 2 elimination half-lives

Heroin $t_{1 / 2}=2-6$ minutes

$6-M A M t_{1 / 2}=2-25$ minutes

Morphine $t_{1 / 2}=1.4-6.7$ hours; $V_{d}=2-5 \mathrm{~L} / \mathrm{kg}$

Codeine $t_{1 / 2}=1.9-3.9$ hours; $V_{d}=3.5 \mathrm{~L} / \mathrm{kg}$

\begin{tabular}{|c|c|c|c|c|c|c|c|c|c|c|}
\hline $\begin{array}{l}\text { AM -Death } \\
\text { (hours) }\end{array}$ & Case\# & $\begin{array}{c}\text { Source } \\
\text { (whole blood) }\end{array}$ & $\begin{array}{c}\text { Concentration } \\
(\mathrm{ng} / \mathrm{mL})\end{array}$ & $\begin{array}{c}\text { AM } \\
\text { concentration } \\
(\mathbf{n g} / \mathbf{m L}) \\
\end{array}$ & Analyte & PM:AM & $C: P$ & $\begin{array}{l}\text { PMI } \\
\text { (hours) }\end{array}$ & Sex & Age \\
\hline \multirow{2}{*}{6.5} & \multirow{2}{*}{$\begin{array}{c}\text { MOC } \\
09^{1}\end{array}$} & Aorta & codeine 34 & \multirow{2}{*}{48 codeine } & $\mathrm{CO}$ & 0.7 & \multirow{2}{*}{1.2} & \multirow{2}{*}{33.5} & \multirow{2}{*}{$\mathrm{BM}$} & \multirow{2}{*}{38} \\
\hline & & Iliac & codeine 27 & & $\mathrm{CO}$ & 0.6 & & & & \\
\hline \multirow{2}{*}{10} & \multirow{2}{*}{$\begin{array}{c}\mathrm{MOC} \\
11^{2}\end{array}$} & Mix heart & morphine 9.5 & \multirow{2}{*}{$\begin{array}{l}92 \text { morphine } \\
17 \text { codeine }\end{array}$} & $\mathrm{MO}$ & 0.1 & \multirow{2}{*}{ - } & \multirow{2}{*}{19.5} & \multirow{2}{*}{$\mathrm{WF}$} & \multirow{2}{*}{34} \\
\hline & & IVC & morphine 8 & & MO & 0.1 & & & & \\
\hline \multirow{2}{*}{$<10$} & \multirow{2}{*}{$\begin{array}{c}\mathrm{MOC} \\
06^{1}\end{array}$} & Aorta & $\begin{array}{c}\text { morphine } 17 \\
\text { codeine } 25\end{array}$ & \multirow{2}{*}{$\mathrm{NDD}^{3}$} & $\begin{array}{l}\mathrm{MO} \\
\mathrm{CO}\end{array}$ & - & \multirow{2}{*}{$\begin{array}{l}1.0 \\
0.9\end{array}$} & \multirow{2}{*}{48} & \multirow{2}{*}{ WM } & \multirow{2}{*}{41} \\
\hline & & Iliac & $\begin{array}{c}\text { morphine } 17 \\
\text { codeine } 27\end{array}$ & & $\begin{array}{l}\mathrm{MO} \\
\mathrm{CO}\end{array}$ & $\begin{array}{l}- \\
-\end{array}$ & & & & \\
\hline \multirow{2}{*}{12} & \multirow{2}{*}{$\begin{array}{c}\mathrm{MOC} \\
15^{2} \\
\end{array}$} & \multirow{2}{*}{ Heart* } & \multirow{2}{*}{ morphine 156} & \multirow{2}{*}{98 morphine } & $\mathrm{MO}$ & 1.5 & \multirow{2}{*}{-} & \multirow{2}{*}{23} & \multirow{2}{*}{ WF } & \multirow{2}{*}{62} \\
\hline & & & & & $\mathrm{MO}$ & 1.7 & & & & \\
\hline \multirow{2}{*}{$\leq 20$} & \multirow{2}{*}{$\begin{array}{c}\mathrm{MOC} \\
04^{1}\end{array}$} & Mix heart & $\begin{array}{c}\text { morphine } 1,170 \\
\text { codeine } 10\end{array}$ & \multirow{2}{*}{298 morphine } & $\mathrm{MO}$ & 3.9 & \multirow{2}{*}{ - } & \multirow{2}{*}{34} & \multirow{2}{*}{$\mathrm{BF}$} & \\
\hline & & IVC & $\begin{array}{c}\text { morphine } 111 \\
\text { codeine } 9.5\end{array}$ & & MO & 0.4 & & & & 65 \\
\hline & $\mathrm{MOC}$ & Heart & $\begin{array}{c}\text { morphine } 20.5 \\
\text { codeine } 14\end{array}$ & Detected ${ }^{4}$ & $\mathrm{MO} / \mathrm{CO}$ & - & & 315 & & \\
\hline 24 & $10^{1}$ & Heart & $\begin{array}{c}\text { morphine } 17 \\
\text { codeine } 14\end{array}$ & Detected & $\mathrm{MO} / \mathrm{CO}$ & - & $\begin{array}{lll}- & \text { r }\end{array}$ & 31.5 & WM & 39 \\
\hline$<2$ & $\mathrm{MOC}$ & Aorta & $\begin{array}{c}\text { morphine } 403 \\
\text { codeine } 35\end{array}$ & & $\begin{array}{l}\mathrm{MO} \\
\mathrm{CO}\end{array}$ & $\begin{array}{l}1.8 \\
1.1 \\
\end{array}$ & 0.2 & 20 & RM & 40 \\
\hline$<24$ & $01^{1}$ & Iliac & $\begin{array}{c}\text { morphine } 2,185 \\
\text { codeine } 72\end{array}$ & 32 codeine & $\begin{array}{l}\mathrm{MO} \\
\mathrm{CO}\end{array}$ & $\begin{array}{l}9.7 \\
2.2\end{array}$ & 0.5 & 20 & BM & 40 \\
\hline
\end{tabular}




\begin{tabular}{|c|c|c|c|c|c|c|c|c|c|c|}
\hline $\begin{array}{l}\text { AM -Death } \\
\text { (hours) }\end{array}$ & Case\# & $\begin{array}{c}\text { Source } \\
\text { (whole blood) }\end{array}$ & $\begin{array}{c}\text { Concentration } \\
(\mathrm{ng} / \mathrm{mL})\end{array}$ & $\begin{array}{c}\mathbf{A M} \\
\text { concentration } \\
(\mathrm{ng} / \mathrm{mL})\end{array}$ & Analyte & PM:AM & $C: P$ & $\begin{array}{c}\text { PMI } \\
\text { (hours) }\end{array}$ & Sex & Age \\
\hline \multirow{2}{*}{ unknown } & \multirow{2}{*}{$\begin{array}{c}\mathrm{MOC} \\
07^{1}\end{array}$} & Iliac & $\begin{array}{c}\text { morphine } 33.5 \\
\text { codeine } 81\end{array}$ & \multirow{2}{*}{10 codeine } & $\mathrm{CO}$ & 8.1 & \multirow[b]{2}{*}{ - } & \multirow{2}{*}{46.5} & \multirow{2}{*}{ WF } & \multirow{2}{*}{43} \\
\hline & & Iliac & $\begin{array}{c}\text { morphine } 30.5 \\
\text { codeine } 42.5\end{array}$ & & $\mathrm{CO}$ & 4.2 & & & & \\
\hline
\end{tabular}

Morphine $=\mathrm{MO}$

Codeine $=\mathrm{CO}$

${ }^{1} \mathrm{GC} / \mathrm{MS}$ linear range $10-1,000 \mathrm{ng} / \mathrm{mL}$

${ }^{2} \mathrm{GC} / \mathrm{MS}^{2}$ linear range $5-1,000 \mathrm{ng} / \mathrm{mL}$

${ }^{3} \mathrm{AM}$ blood was negative, but qualitative screen of urine was positive for morphine

${ }^{4}$ Sample quantity only sufficient for blood drug screen, not quantitation

* Average concentration

\section{Table10, continued}


morphine concentration was $149 \mathrm{ng} / \mathrm{mL}$, while the gray-top equaled $164 \mathrm{ng} / \mathrm{mL}$. Statistical analysis determined the PM morphine concentrations were similar. Since these samples were statistically similar and of the same source their morphine concentrations were averaged to equal $156 \mathrm{ng} / \mathrm{mL}$. The PM results not only exceed their expected range, they also surpassed the AM concentration, resulting in a HTBL:AM ratio of 1.6. The data supports the idea that PMR occurred during the 23 hour postmortem interval.

Antemortem blood for MOC 04 had $298 \mathrm{ng} / \mathrm{mL}$ of morphine present. Codeine was not detected in the sample. The AM tube did not indicate the time of collection; therefore, the exact time between the AM collection and death was not known. However, based on the hospital records the decedent was in the hospital for 20 hours before death, therefore the AM sample would have been collected within the same time frame. When using 20 hours as the time between AM collection and death, the expected morphine concentration at the time of death would have been between $0.15-37.7 \mathrm{ng} / \mathrm{mL}$. Postmortem mixed heart (MXHT) and IVC bloods were submitted for analyses. The MXHT blood sample had $1,170 \mathrm{ng} / \mathrm{mL}$ of morphine and $10 \mathrm{ng} / \mathrm{mL}$ of codeine. One hundred - eleven nanograms per milliliter of morphine and $9.5 \mathrm{ng} / \mathrm{mL}$ of codeine were detected in the IVC blood sample. Both PM morphine concentrations exceeded their expected ranges. Morphine PM:AM ratios were calculated to be 3.9 and 0.37 for mixed heart and IVC bloods, respectively. Codeine, which was not detected in the AM sample, was present in both PM samples, near the LOQ of $10 \mathrm{ng} / \mathrm{mL}$ (GC- SIM/MS) detection. The increased codeine concentration did suggest PMR of codeine. While the ratio data for morphine in the MXHT sample was indicative of PMR, it's concentration in the IVC BL sample did not. However, both PM morphine concentrations exceeded the calculated 
concentration at the time of death. Therefore, the researcher determined that both PM morphine values were a result of PMR.

The volume of AM blood submitted for MOC 10 was only sufficient for morphine identification (codeine was not detected) in a routine blood screen. Before quantitative analysis could be conducted, the sample was depleted. Heart blood was submitted in both gray-top and red-top tubes. Analysis of the RT tube detected $20.5 \mathrm{ng} / \mathrm{mL}$ of morphine and $14 \mathrm{ng} / \mathrm{mL}$ of codeine. Gray-top tube analysis detected 17 $\mathrm{ng} / \mathrm{mL}$ of morphine and $14 \mathrm{ng} / \mathrm{mL}$ of codeine. Since morphine was not quantitated in the AM sample, it is not known how the 31.5 hour PMI affected the sample concentration, if at all. The emergence of codeine in the PM does suggest PMR for that drug. Additionally, the consistent drug concentrations between the two tubes shows how similar results can be obtained when a sample is properly homogenized with the antimicrobial agent present within the tubes.

Morphine/codeine 01 had antemortem concentrations of $226 \mathrm{ng} / \mathrm{mL}$ and $32 \mathrm{ng} / \mathrm{mL}$ for morphine and codeine, respectively. As a result, the expected concentration range for morphine was $0.001-18.9 \mathrm{ng} / \mathrm{mL}$, while codeine was $0.005-0.45 \mathrm{ng} / \mathrm{mL}$ by $\mathrm{GC} / \mathrm{MS}$ $(\mathrm{LOD}$ and $\mathrm{LOQ}=10 \mathrm{ng} / \mathrm{mL})$. Postmortem analysis was conducted on aorta and iliac vein blood samples. The aorta blood had concentrations of $403 \mathrm{ng} / \mathrm{mL}$ of morphine and $35 \mathrm{ng} / \mathrm{mL}$ of codeine. The iliac vein blood sample had a morphine concentration of 2,185ng/mL and codeine of $72 \mathrm{ng} / \mathrm{mL}$. The PM concentrations of both morphine and codeine exceeded the antemortem concentrations and calculated concentration ranges at the time of death. Aorta blood to AM ratio equaled 1.8 and 1.1 for morphine and codeine, respectively. The iliac blood to AM ratio equaled 9.7 and 2.25 for morphine and 
codeine, respectively. All of the PM:AM ratios were consistent with PMR being a factor in this case. However, since the iliac vein blood had higher concentrations than the aorta blood, the C:P ratios for both morphine and codeine were below 1.0. Regardless, the increased PM concentrations were enough evidence to support the possibility of PMR being present within this case.

Alternatively, while heroin was not detected in MOC 01's biological samples, the significant difference between morphine and codeine blood concentrations for MOC 01 suggested the possibility of heroin usage. A review of the circumstances surrounding the decedent's death indicated that his body was found with a syringe in close proximity. If heroin was injected in the leg or foot area, this would account for the elevated morphine concentration in the iliac vein blood as compared to the aorta blood. Under those circumstances, heroin would have metabolized out of the blood stream, but morphine and codeine remained. If this were true, then incomplete drug distribution, not PMR, was the reason for the concentration differences.

Ten nanograms per milliliter of codeine were detected in the AM sample collected for MOC 07. In this case, the exact time of the AM sample before death was unknown. Based on the medical records obtained by the MDME Department, the decedent was hospitalized for three days before her death. Postmortem iliac vein blood was submitted in both gray-top and red-top tubes for analyses. The RT sample had morphine and codeine concentrations of 35.5 and $81 \mathrm{ng} / \mathrm{mL}$, respectively. The GT sample had $30.5 \mathrm{ng} / \mathrm{mL}$ of morphine and $42.5 \mathrm{ng} / \mathrm{mL}$ of codeine detected. Since morphine is a metabolite of codeine, its presence in the PM samples and absence in the AM sample, may suggest that metabolism continued between the time of AM sample 
collection and death. However, since both morphine and codeine concentrations were elevated in the PM samples, it is also likely the PMR occurred for both drugs. The inconsistent codeine concentration within the RT and GT iliac vein blood samples could be indicative of two separate blood draws. In instances such as this, one draw will be used to fill the red-top tube (which can hold up to $50 \mathrm{~mL}$ of blood), while a second draw can be used to fill the gray-top tube ( $10 \mathrm{~mL}$ total volume). If two draws occurred, then it is possible that the blood was drawn from two different regions of the iliac vein, which resulted in the significant codeine concentration discrepancy between the two tubes.

Those cases whose antemortem collection were greater than or equal to two elimination half-lives add value to this section by highlighting the importance of time. In the time between antemortem blood draw and death, drug concentration and identification can be altered. In morphine/codeine case 09 , the 6.5 hour interval between AM and death was sufficient time for codeine (which was detected in both the AM and PM samples) to metabolize, resulting in a lower concentration. While the PM concentration did decrease, it still exceeded its expected concentration range, which was believed to be due the PMR that occurred during the 33.5 hour PMI.

In comparison, morphine/codeine case 11 had AM samples collected 10 hours before death, but had a PMI of 19.5 hours. Therefore, the time difference between AM to death increased, but the PMI decreased when comparing it to MOC 09. In MOC 11, both morphine and codeine were detected in the AM, but only morphine in the PM sample. It is believed that in this case, codeine metabolized to concentrations below that of detection. Morphine decreased as well, to a concentration that would be expected of a drug that metabolized in a linear fashion. While it cannot be said for certain, the 
differences in PMI (14 hours) may have made the difference in these two cases. If the PMI of MOC 09 had been shorter or the PMI of MOC 11 had been longer, the results may not have been the same.

Morphine/codeine 06 had an extended PMI of 48 hours. In this case, neither morphine nor codeine was detected in the AM sample. However, they were both detected in the PM samples. This suggests that PMR was a factor here and the PMI may have contributed to this. This was also seen in MOC 04 where only morphine was detected in the AMBL, but both morphine and codeine were detected in the PM blood samples (PMI $=34$ hours).

Morphine/codeine 04 was also of interest because it illustrated the significant concentration differences that can be present within different sourced PM blood samples. In this case, a mixed heart sample had a 10 fold increase in morphine concentration over the IVC BL sample. The mixed heart sample, which was blood collected from more than one unspecified region of the heart, is susceptible to contamination from both the liver and the lungs. The inferior vena cava blood is typically drawn from the lower abdominal region of the body (52). While both samples are considered central sourced blood, their anatomical location can result in very different drug concentrations.

Morphine/codeine 01 was of interest because the peripheral source blood (ILBL) greatly exceeded the concentration of both the aorta and antemortem bloods. The reason for this large increase cannot be definitively determined. Based on the other MOC cases examined here, there is no reason to believe that the 20 hour PMI was sufficient to cause such a great concentration inconsistency. While it is understood that drug concentrations do not always respond in a predictable manner, it would seem that this case would be an 
exception to the rule. An alternative explanation would suggest that the drug was injected intravenously into the leg or foot region of the body. This would account for the significantly higher morphine concentration found in the iliac vein blood.

An additional factor in blood concentration, regardless of half-life time intervals would be the anatomic location of blood draws. In MOC 07, iliac vein blood was submitted in both blood collection tubes. In situations like this, it is expected that blood concentrations from either tube would be comparable to each other. However, the codeine concentration in the red-top tube was almost twice that of the gray-top. This could have suggested that the blood drawn from both tubes were either collected from different regions of the same iliac vein or collected from different iliac veins all together (i.e. left and right leg veins). Either scenario is plausible, as the sample volume from the iliac vein is relatively limited. Similarly, the HTBL sample submitted in both RT and GT tubes for MOC 10 had a morphine concentration discrepancy. While the difference in the drug concentration seen here was $<20 \%$, it was greater than the significance limit ( \pm $10 \%$ ). This shows that even a relatively abundant source of blood (as compared to the iliac vein) may not offer a homogenous sample. Conversely, since the morphine heart blood concentrations for MOC 10 were statistically similar ( $\pm 9.1 \%$ by GC/MS) the results were averaged. The data produced by these three cases indicates that while one would like to believe that same source samples collected at the same time would render statistically similar results, it is definitely not guaranteed.

And finally, a debatable issue in PM toxicology is whether or not peripheral blood sources most closely resemble the blood drug concentration around the time of death. Chart 3 displays the peripheral results for both morphine and codeine. Each chart also 
displays the calculated expected concentration range, denoted with the symbol "[" for morphine and "]" for codeine. In MOC 01, the morphine sample concentration $(2,185 \mathrm{ng} / \mathrm{mL})$ was not fully displayed, as it would have made it difficult to see the results of the cases with the lower concentrations. Additionally, MOC 06 did not have an expected range because morphine was not detected in the AM sample. Likewise, MOC cases 14, 08 and 06 did not have expected codeine ranges, as codeine was not identified in their respective AM samples. Also, MOC 07 has two codeine results displayed. This was because codeine was detected in both RT and GT tubes; however, their concentrations were not similar enough to be averaged into one. As a result, the data from both tubes is represented.

Of the cases that had calculated expected ranges, only one (MOC 08) had its morphine concentration within the expected range. The other cases, whether it be for morphine or codeine, had peripheral concentrations that significantly exceeded their expected values. This indicated that for morphine and codeine cases, peripheral blood results are not likely to be reflective of perimortem blood concentrations.

One additional note of interest was how the central and peripheral blood concentrations compared to each other. Chart 4 and Chart 5 show the central versus peripheral data for morphine and codeine, respectively. Again, the peripheral morphine concentration for MOC 01 was truncated so that the data from the other cases would be visible. When examining the morphine data, only MOC 06 had central and peripheral data that rendered statistically similar results. Of the five codeine cases, only MOC 08 and MOC 06 had statistically similar intra-case central and peripheral results. Among the remaining dis-similar results, $66 \%$ of the morphine cases had higher central sourced 


\section{Chart 3: Morphine and Codeine Peripheral Data}

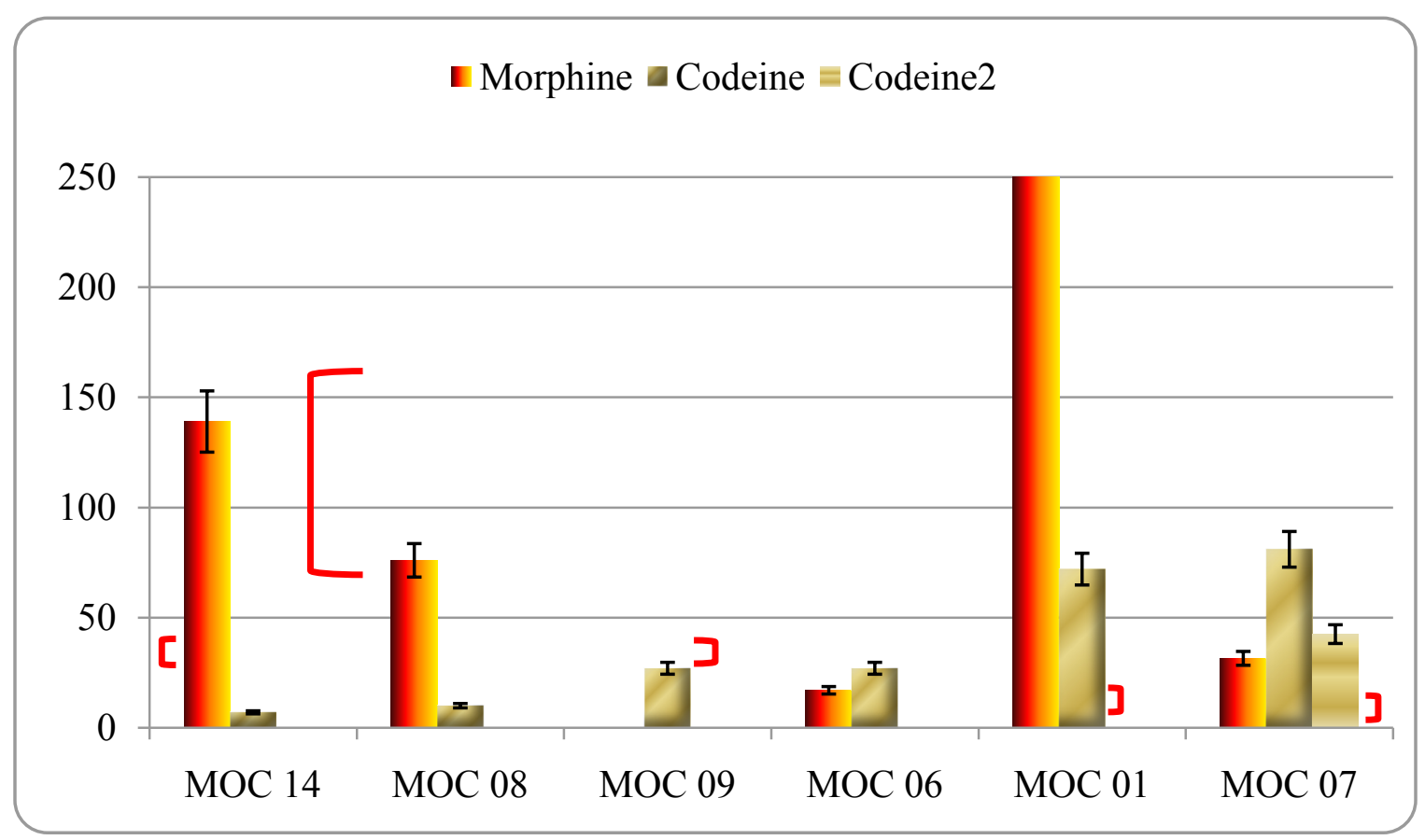

Comparison of the peripheral concentrations to the expected concentration range at the time of death 


\section{Chart 4: Morphine Central versus Peripheral Data}

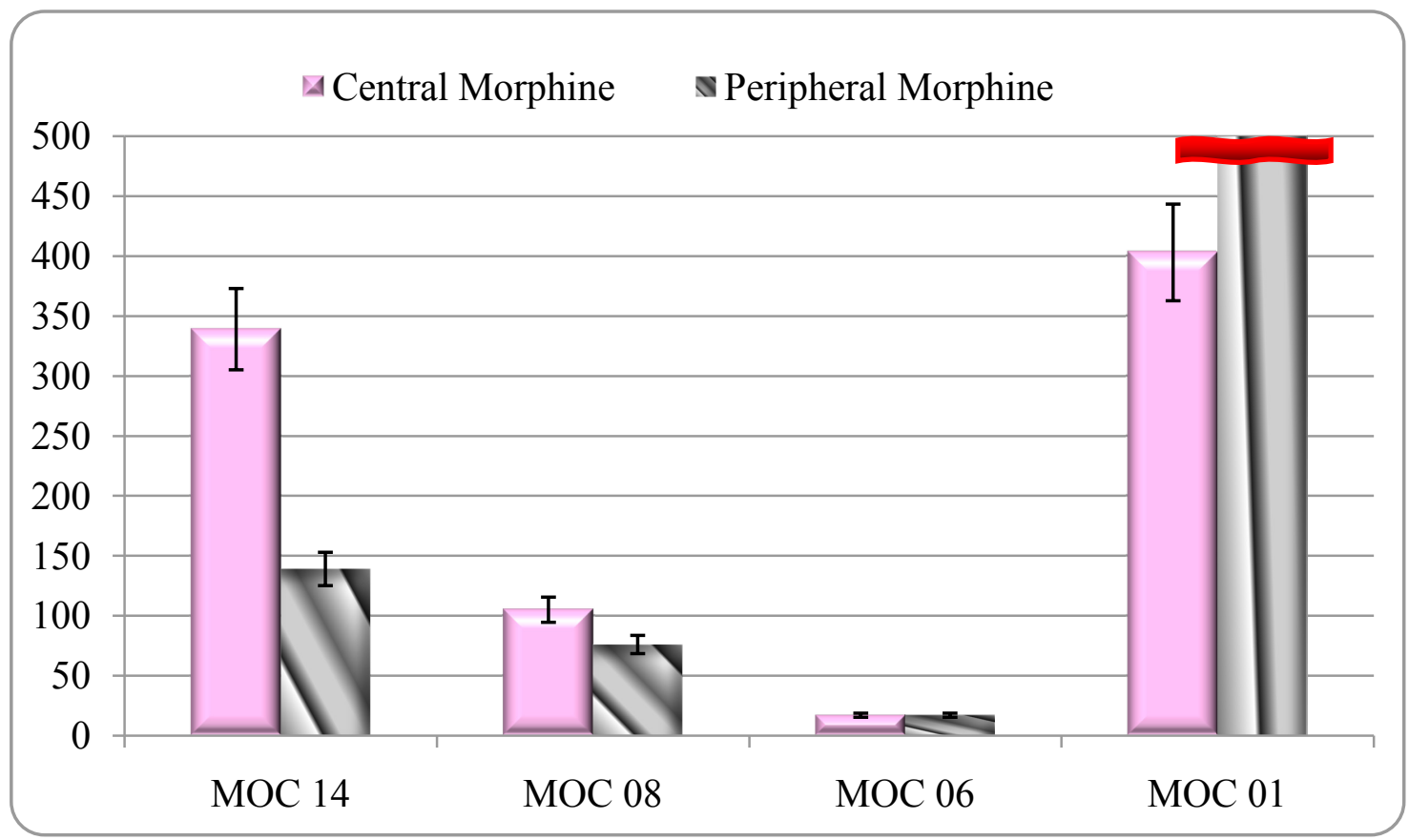

Chart 5: Codeine Central versus Peripheral Data

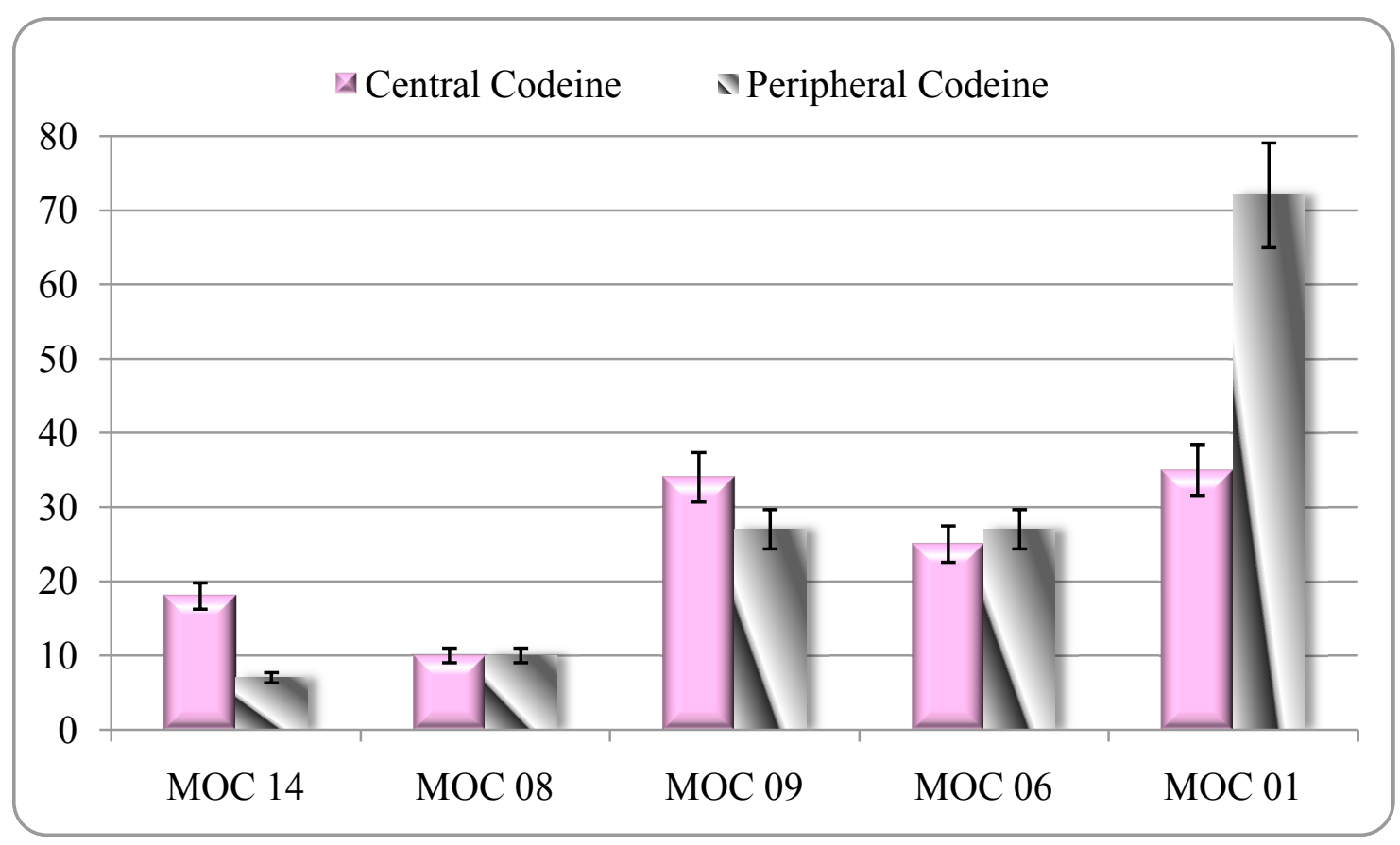


concentrations, while $66 \%$ of codeine did as well. Based on this data, it would appear that among statistically dis-similar data, peripheral blood results would likely offer drug concentrations with fewer instances of increase. However, as indicated by Chart 3, peripheral blood results still may not render drug concentrations that are indicative of drug levels in the body at the time of death.

\subsubsection{Methadone}

Methadone, which is often used for the treatment of opioid addiction, is a synthetic opioid whose plasma half-life ranges from 15 to 55 hours (42). It has an apparent volume of distribution $\left(\mathrm{V}_{\mathrm{d}}\right)$ of $4-5 \mathrm{~L} / \mathrm{kg}(42)$. In total, six cases were examined in order to establish a drug concentration trend between antemortem and postmortem samples. This section was divided into two parts, with both sections listing the cases in order of increasing antemortem to death interval, as opposed to case number. The first section encompasses those cases whose AM samples were collected within one elimination half-life (Table 13). The second data set includes those cases where the AM blood was collected longer than one elimination half-life before death (Table 14). As discussed in section 1.4.3, half lives measure the amount of time needed for an ingested substance to be removed from the system. With each half-life accounting for a $50 \%$ decrease in concentration, it is estimated that at the end of five to six half-lives, the body will rid itself of a drug in its entirety. Depending on the administered concentration and the detection limits of an instrument, methadone taken within 4 half-lives $(60-220 \mathrm{hrs})$ of death, could still be present at detectable, if not quantifiable concentrations. 
Methadone analysis was conducted solely on GC/NPD. All samples were prepared as discussed in Section 4.3 Basic Drugs: Quantitative Blood Analysis by $G C / N P D$. The controls, which were run thirty-one times, had two lot numbers. A oneway ANOVA $(\alpha=0.05)$ was conducted on the two lots to determine if the data produced within them was statistically similar. The summary results shown in Table 11 give the breakdown information (including control averages and variances) for each lot. The ANOVA results (Table 12) give an F-test value of 1.28 and an $F_{\text {crit }}$ of 4.18. Since F is less than $\mathrm{F}_{\text {crit, }}$ the two lots were determined to be statistically similar, resulting in the methadone data being treated as one population. The percent error (CV) was calculated to be $12.52 \%$. Therefore, only drug concentrations that differ beyond that percentage were considered significantly different.

Antemortem serum and postmortem iliac vein blood were received for MDN 04. Initial laboratory analysis determined that the AM serum (collected 45 minutes before death) was negative for methadone. Postmortem analysis conducted by the researcher determined that methadone was present in the iliac vein blood samples. Iliac vein blood received in both vacutainer and red-top polypropylene tubes were positive for methadone, each with a concentration of $0.1 \mathrm{mg} / \mathrm{L}$. Since the PM samples were of the same source, their concentrations were averaged (average $=0.1 \mathrm{mg} / \mathrm{L}$ ). In this case, the methadone was self administered (as opposed to being given in the hospital). Based on the low PM concentrations, it can be inferred that either a small dose of methadone was ingested shortly before death (less than 48 hours) or a larger dose was taken more than 48 hours before death. In either instance, the absence of methadone in the AM sample and 
Table 11: One-Way ANOVA Summary Results for Methadone Controls $(0.50 \mathrm{mg} / \mathrm{L})$ $n=31$

\begin{tabular}{|ccccc|}
\hline Groups & Count & Sum & Average & Variance \\
\hline Lot 1 & 14 & 6.80 & 0.48 & 0.003457 \\
\hline Lot 2 & 17 & 8.60 & 0.50 & 0.001563 \\
\hline
\end{tabular}

Table 12: One-Way ANOVA Results for Methadone Controls 95\% Confidence Limit

\begin{tabular}{|ccccccc|}
$\begin{array}{c}\text { Source of } \\
\text { Variation }\end{array}$ & $\begin{array}{c}\text { Sum of } \\
\text { Squares }\end{array}$ & $d f$ & $\begin{array}{c}\text { Mean } \\
\text { Square }\end{array}$ & $\begin{array}{c}\text { F-test } \\
\text { value }\end{array}$ & P-value & F crit \\
\hline Between Lots & 0.003 & 1 & 0.003 & 1.28 & 0.266 & 4.18 \\
\hline
\end{tabular}

$>$ If $\mathrm{F}>\mathrm{F}$ critical, then results are significantly different

$>$ If $\mathrm{F}<\mathrm{F}$ critical, then results are similar 
quantifiable presence of it in the PM samples, suggested the presence of PMR (PMI=28.5 hours).

Methadone (MDN) case 01 was received with antemortem blood collected eight hours before death and a postmortem interval (PMI) of 23 hours. The AM sample had a methadone concentration of $0.26 \mathrm{mg} / \mathrm{L}$. Postmortem specimens including central (mixed heart) and peripheral (iliac vein) blood sources, had concentrations of $0.53 \mathrm{mg} / \mathrm{L}$ and $0.58 \mathrm{mg} / \mathrm{L}$, respectively. When using Equation 7, the expected concentration range at the time of death would have been between $0.19 \mathrm{mg} / \mathrm{L}$ to $0.24 \mathrm{mg} / \mathrm{L}$. However, the results determined by PM analysis were more than twice that. A review of the medical records for this case did not indicate any additional methadone dosing after the time of AM collection to account for the increase in drug concentration detected in the PM samples.

An 8 hour AMD interval, as seen in MDN 01, is less than the minimum amount of time needed for one elimination half-life (15 hours). However, metabolism was still occurring. Therefore, the drug concentrations detected in the PM samples should have been lower than the AMBL concentration. Instead, the PM concentrations showed a substantial increase. The increased PM blood concentrations suggested postmortem release of methadone from the tissues that surrounded the blood collection sites at autopsy. The ratio of mixed heart:AM was 2.0 and iliac vein:AM was 2.2. Additionally, the mixed heart:iliac vein ratio was calculated to be 0.9 . Independent studies have demonstrated $(19)(20)(21)$ that central, rather than peripheral, blood sources are more susceptible to PMR. Because of this, it is often believed that peripheral blood sources offer more reliable data than central sources. However, in this case, less than $10 \%$ separated the concentration of the two sources. The central $(0.53 \mathrm{mg} / \mathrm{L})$ and peripheral 
$(0.58 \mathrm{mg} / \mathrm{L})$ concentrations were statistically similar, indicating that both sources were equally susceptible to the PM redistributive effect.

The decedent in MDN 05 died during a surgical procedure. Upon admission into the hospital he acknowledged his use of cocaine and heroin. The hospital released the AM blood collected prior to surgery to the Medical Examiner's Department. Analyses detected the presence of hospital administered medications (e.g. phenobarbital and lidocaine), as well as non-hospital administered drugs such as cocaine, benzoylecgonine (a major metabolite of cocaine), morphine (a metabolite of heroin, as well as a prescribed pain medication) and hydromorphone. Unfortunately, by the end of this testing, there was not enough AM blood remaining to test for the presence of methadone. Laboratory testing did include a urine analysis (qualitative screen), which was positive for methadone. Subsequent quantitative analysis by the laboratory was conducted on PM blood in lieu of AM blood.

Routine laboratory analysis of the chest blood submitted for MDN 05 (PMI $=16$ hours) detected $0.26 \mathrm{mg} / \mathrm{L}$ of methadone. Medical records indicated that methadone was administered by the hospital 2.5 hours before death. One month after routine laboratory testing, the researcher re-analyzed both postmortem chest blood tubes (red and gray). Each had a concentration of $0.25 \mathrm{mg} / \mathrm{L}$, since the PM samples were of the same source their concentrations were averaged $(0.25 \mathrm{mg} / \mathrm{L})$. The lack of AM testing meant that this case could not be evaluated for differences between AM and PM drug concentrations; however it is an example of another goal of the project. While it is understood that one sample is not a relevant population, the re-analysis suggested that when a preserved methadone sample is stored in refrigeration for 30 days, degradation may not be a factor. 
Drug stability is an important variable to consider when trying interpreting the meaning of toxicological data.

Methadone (MDN) 02 was submitted for laboratory testing with AM blood collected 12 hours before death. The postmortem submission consisted of both aorta and iliac vein bloods ( $\mathrm{PMI}=54$ hours). Routine analysis determined that the $\mathrm{AM}$ concentration of methadone was $2.3 \mathrm{mg} / \mathrm{L}$. Subsequent analysis of the PM samples determined concentrations of $5.32 \mathrm{mg} / \mathrm{L}$ and $4.62 \mathrm{mg} / \mathrm{L}$ for the aorta (AOBL) and iliac vein (ILBL) bloods, respectively. The linear range of methadone for this project was from $0.1-1.0 \mathrm{mg} / \mathrm{L}$. Therefore, the samples were diluted to bring their concentrations within the appropriate range. The decedent had a history of opiate abuse (specifically, heroin and morphine). However, none of the records available to the researcher suggested that the decedent was receiving methadone treatment to help alleviate the withdrawal symptoms associated with opiate addiction. Typically people receiving such treatment are given daily doses ranging from $100-200 \mathrm{mg}$ (42). Studies have shown that when following this dosing regimen, the average peak plasma concentration is $0.83 \mathrm{mg} / \mathrm{L}(42)$. The concentrations encountered in MDN 02, well exceeded this level. Based upon the AM concentration of $2.3 \mathrm{mg} / \mathrm{L}$, the expected concentration range at the time of death would have been between $1.38-2.05 \mathrm{mg} / \mathrm{L}$. The calculated ratios of AOBL:AM and ILBL:AM was 2.3 and 2.0, respectively. The central to peripheral ratio of AOBL:ILBL equaled 1.1. All three of the ratio calculations were greater than 1.0, suggesting that PMR occurred during the 54 hour PMI. However, while the aorta blood appeared to receive a larger redistribution effect than the iliac vein blood, the differences in their concentrations were not statistically significant. The significant range for the 
Table 13: Methadone Antemortem samples collected within one elimination half-life

$$
\begin{gathered}
t_{1 / 2}=15-55 \text { hours } \\
V_{d}=4-5 \mathrm{~L} / \mathrm{kg} \\
C V=12.5 \%
\end{gathered}
$$

\begin{tabular}{|c|c|c|c|c|c|c|c|c|c|c|}
\hline $\begin{array}{c}\text { AM- } \\
\text { Death }\end{array}$ & Case \# & $\begin{array}{c}\text { Source } \\
\text { (Whole blood) }\end{array}$ & $\begin{array}{c}\text { Concentration } \\
(\mathrm{mg} / \mathrm{L})\end{array}$ & $\begin{array}{c}\text { AM } \\
\begin{array}{c}\text { Concentration } \\
(\mathrm{mg} / \mathrm{L})\end{array} \\
\end{array}$ & Specimen & $\begin{array}{l}\text { PM: } \\
\text { AM }\end{array}$ & C: P & $\begin{array}{c}\text { PMI } \\
\text { (hours) }\end{array}$ & Race/Sex & Age \\
\hline $45 \mathrm{~min}$ & MDN 04 & Iliac* & 0.10 & Not detected & $\begin{array}{c}\text { AM } \\
\text { Blood }\end{array}$ & - & - & 28.5 & WM & 44 \\
\hline \multirow{2}{*}{$8 \mathrm{hrs}$} & \multirow{2}{*}{ MDN 01} & Mixed heart & 0.53 & \multirow{2}{*}{0.26} & \multirow{2}{*}{$\begin{array}{c}\text { AM } \\
\text { Blood }\end{array}$} & 2.0 & \multirow{2}{*}{0.91} & \multirow{2}{*}{23} & \multirow[t]{2}{*}{ WM } & \multirow{2}{*}{33} \\
\hline & & Iliac & 0.58 & & & 2.2 & & & & \\
\hline $12 \mathrm{hrs}$ & MDN 05 & Chest* & 0.25 & 0.26 & $\begin{array}{l}\text { PM }^{1} \\
\text { Blood }\end{array}$ & 0.96 & - & 16 & WM & 38 \\
\hline \multirow{2}{*}{$12 \mathrm{hrs}$} & \multirow{2}{*}{ MDN 02} & Aorta & 5.32 & \multirow{2}{*}{2.3} & \multirow{2}{*}{$\begin{array}{c}\text { AM } \\
\text { Blood }\end{array}$} & 2.3 & \multirow{2}{*}{1.1} & \multirow{2}{*}{54} & \multirow{2}{*}{ WM } & \multirow{2}{*}{68} \\
\hline & & Iliac & 4.62 & & & 2.0 & & & & \\
\hline
\end{tabular}

Linear range: $0.1-1.0 \mathrm{mg} / \mathrm{L}$

${ }^{\mathrm{T}}$ Quantity not sufficient for antemortem quantitation

*Average concentration 
AOBL was $4.65-5.98 \mathrm{mg} / \mathrm{L}$, while ILBL was $4.04-5.20 \mathrm{mg} / \mathrm{L}$. The critical ranges for the two samples overlap, indicating that they are not statistically different.

When comparing these four cases, there were a few observations to note. First, methadone concentrations were stable regardless of the collection tube. In MDN 05 chest blood was collected in both tubes, while MDN 04 had iliac vein blood in both tubes. Regardless of the anatomical source or collection tube, the blood drug concentration of methadone was stable when stored under the same conditions.

Additionally by convention, toxicologists prefer to use peripheral blood for quantitative analysis. Out of the four cases examined, two (MDN 01 and MDN 02) were received with both central and peripheral blood. Both MDN 01 and 02 were affected by PMR and their intra-sample results were statistically similar. This meant, that despite the region of anatomical collection, the PM results returned analogous results. While there are not enough cases available to make any absolute decisions, it would appear that regardless of the PMI (MDN $01=23$ hrs., MDN $02=54$ hrs.), PMR of methadone can affect central and peripheral blood sources similarly.

Finally, the overall data collected from these four cases illustrated that AM methadone concentrations are consistently lower than their PM counterparts. This trend was observed in all of the quantifiable data produced.

Included in the second part of this section are two methadone cases (Table 14). One case had an AM collection of 72 hours before death. While this amount of time may seem significant, in actuality it is within two elimination half-lives of methadone. As a result, it is reasonable to believe that PM drug analysis could still be positive for methadone. The second case had an AM collection of 20 hours after death. Blood 
samples can be collected after brain death for patients who are being maintained artificially for the purpose of organ donation.

The decedent in MDN 03 was found unresponsive at home by a family member. He had a history of heart disease and heroin addiction. He sought out treatment for his addiction and was prescribed a methadone treatment regimen of 80 milligrams per day. The hospital submitted AM Blood collected 72 hours prior to his death. The AM methadone concentration was $0.7 \mathrm{mg} / \mathrm{L}$, resulting in an expected concentration range at the time of death of $0.02-0.28 \mathrm{mg} / \mathrm{L}$. Postmortem analysis $(\mathrm{PMI}=24.5$ hours $)$ detected methadone concentrations of 1.06 and $0.51 \mathrm{mg} / \mathrm{L}$ for aorta and inferior vena cava (IVC) bloods, respectively. While both AOBL and IVC BL are commonly considered central sourced bloods, the data they produced were statistically significantly different. In situations like this, it becomes increasingly important to identify the actual region of collection for the IVC BL.

The inferior vena cava is the largest vein in the body. It begins at the joining of the common iliac vein and continues to the right atrium, where it delivers deoxygenated blood (53). Contamination of the IVC, depends greatly upon the region of collection. Conversely, the aorta's primary function is to distribute oxygenated blood away from the heart and to the other tissues of the body. The aorta is comprised of three continuous parts (ascending, arch and descending components), which based on its location can receive direct contamination from both lungs, as well as the pulmonary artery. Upon investigating the source of the IVC BL, it was explained that at the MDME Department, IVC blood is collected from the lower abdominal region of the body (52). This means that the IVC BL is peripherally located in comparison the AOBL. When examining the 
data, the AOBL:AM ratio equaled 1.5, while the IVC BL:AM ratio equaled 0.73 . According to this ratio data, no PMR was observed in the IVC BL sample. However, the AOBL ratio of 1.5 was indicative of PMR. These results do follow the conventional idea that more centrally located blood specimens are more susceptible to PMR.

The decedent in methadone 06 was an organ donor; whose AM sample was collected 20 hours after brain death (refer to section 1.6.5). Upon analysis, the AM blood was negative of methadone. The postmortem aorta and iliac vein bloods however had methadone concentrations of $0.15 \mathrm{mg} / \mathrm{L}$ and $0.10 \mathrm{mg} / \mathrm{L}$, respectively. With a methadone $\mathrm{CV}$ of $12.52 \%$ the critical ranges for the AOBL and ILBL concentrations were $0.13-$ $0.17 \mathrm{mg} / \mathrm{L}$ and $0.09-0.11 \mathrm{mg} / \mathrm{L}$, respectively thereby making these concentrations statistically dis-similar. The AOBL:ILBL ratio equaled 1.5. The ratio data suggested that during the 31.5 hour postmortem interval, drug redistribution occurred. This redistribution effect was more pronounced in the aorta blood, rather than iliac vein blood. The ratio data, along with the lack of drug detection in the AM sample, but its presence in both PM samples indicated that PMR was a factor in this case.

When examining all six cases as a whole it became apparent that the methadone data could have been grouped as it was (by increasing antemortem to death interval) or based upon their blood collection sites. Three of the cases examined were submitted with both peripheral and central blood, plus the addition of MDN 03, which had a more peripherally located IVC BL and the more centrally located AOBL, as well. One case came with central blood only. Typically blood collected from the central vessels is more abundant than that available from the periphery. For that reason, it is not unusual to receive more central only, rather than peripheral only cases. Of the three cases with both 
Table 14: Methadone Antemortem samples collected after one elimination half-life

$$
\begin{gathered}
t_{1 / 2}=15-55 \text { hours } \\
V_{d}=4-5 \mathrm{~L} / \mathrm{kg} \\
C V=12.5 \%
\end{gathered}
$$

\begin{tabular}{|c|c|c|c|c|c|c|c|c|c|c|}
\hline $\begin{array}{l}\text { AM - } \\
\text { Death }\end{array}$ & Case \# & $\begin{array}{c}\text { Source } \\
\text { (whole blood) }\end{array}$ & $\begin{array}{c}\text { Concentration } \\
(\mathrm{mg} / \mathrm{L})\end{array}$ & $\begin{array}{c}\mathrm{AM} \\
\text { concentration } \\
(\mathrm{mg} / \mathrm{L})\end{array}$ & Specimen & PM:AM & $C: P$ & $\begin{array}{c}\text { PMI } \\
\text { (hours) }\end{array}$ & Age & Race/Sex \\
\hline \multirow{2}{*}{$\begin{array}{c}20 \mathrm{hrs} \\
\text { AFTER }^{1}\end{array}$} & \multirow{2}{*}{$\begin{array}{c}\text { MDN } \\
06 \\
\end{array}$} & Aorta & 0.15 & \multirow{2}{*}{ Not detected } & \multirow{2}{*}{$\begin{array}{c}\text { AM } \\
\text { Blood }\end{array}$} & - & \multirow{2}{*}{1.5} & \multirow{2}{*}{31.5} & \multirow{2}{*}{38} & \multirow{2}{*}{$\mathrm{BM}$} \\
\hline & & Iliac & 0.10 & & & - & & & & \\
\hline \multirow[b]{2}{*}{3 days } & \multirow{2}{*}{$\begin{array}{c}\text { MDN } \\
03\end{array}$} & Aorta & 1.06 & \multirow[b]{2}{*}{0.7} & \multirow{2}{*}{$\begin{array}{c}\text { AM } \\
\text { Blood }\end{array}$} & 1.5 & \multirow[b]{2}{*}{-} & \multirow[b]{2}{*}{24.5} & \multirow[b]{2}{*}{60} & \multirow[b]{2}{*}{ WM } \\
\hline & & $\begin{array}{l}\text { Inf. Vena } \\
\text { Cava }\end{array}$ & 0.51 & & & 0.7 & & & & \\
\hline
\end{tabular}

Linear range: $0.1-1.0 \mathrm{mg} / \mathrm{L}$

AFTER denotes collection time after brain death 
central and peripheral sources, all four had higher PM, rather than AM concentrations. Among those PM concentrations the two cases whose AMD interval exceeded one elimination half-life not only had intra-sample concentrations significantly different from each other, but they also had higher central sourced blood concentrations. Neither of these factors was observed in the cases collected within one elimination half-life.

Those cases submitted with central only bloods (as well as the lone case of peripheral only blood) had PM concentrations much higher than their AM counterparts. The combined effect of all the data showed that regardless of the AMD interval or the PM interval, PMR of methadone can occur. However, the data trend suggested that those AM samples that were collected within one elimination half-life before death could have had a PM redistributive effect that influenced two anatomically different PM sources similarly. This was reiterated by the lack of statistically different concentrations obtained in those cases that had both central and peripheral blood collected. In most instances PM concentrations can greatly exceed both the AM concentration and the expected concentration range at the time of death, as long as the autopsy is conducted in less than 54 hours, it may not matter which PM sample is used for quantitation.

\subsection{Diphenhydramine}

Diphenhydramine is classified as an antihistamine, but has a much wider array of applications. It does relieve allergy related symptoms (e.g. itchy, irritated, watery eyes and sneezing) (54) but is often also utilized for its sedative and antiemetic properties. Over-the-counter formulations of diphenhydramine can range in concentration from $10-$ 
$50 \mathrm{mg}$ doses and are obtained as elixirs, pills or creams (55). In high doses $€ 300 \mathrm{mg}$ ) it has been known to produce psychoactive delusions. This, in combination with its ease of accessibility, has made this drug particularly appealing to adolescents (56).

During the course of this project, three separate diphenhydramine controls (concentration $=0.5 \mathrm{mg} / \mathrm{L}$; each with their own lot numbers) were made. The control values produced for each lot were analyzed using a one-way ANOVA $(\alpha=0.05)$ to determine if a significant variation existed among the collected data. The summary data, produced by Microsoft Excel, displayed in Table 15 shows the average concentrations, as well as the amount of variance present within each lot. The ANOVA results in Table 16 gave an F-test value of 2.39 and a critical $F\left(F_{\text {crit }}\right)$ value of 3.24. Since $F<F_{\text {crit }}$ the data produced by the 3 lots was considered statistically similar, which allowed the data was treated as one population. Equation 5 was used to calculate the coefficient of variation for the total population. This value was determined to be $15 \%$. Therefore, any quantitative diphenhydramine case data that fell within a $\pm 15 \%$ range was considered similar. Data that exceeded these limits were considered dis-similar.

On average it takes diphenhydramine 3-14 hours for one elimination half-life to occur. The twelve cases that are presented here were separated into two sections. The first encompasses those cases whose antemortem bloods were collected within two elimination half-lives before death (Table 17). While the second section, includes those cases that exceed this limit (Table 18). In both sections, the cases were evaluated in the order of increasing antemortem to death (AMD) interval, as opposed to numerical case order. All samples were prepared as described in Section 4.3 Basic Drugs: Quantitative Blood Analysis by GC/NPD (linear range $0.1-1.0 \mathrm{mg} / \mathrm{L}$ ). While the sedative affects of 
diphenhydramine may have contributed to some of the deaths discussed here, it was never listed as the sole cause of death.

Diphenhydramine (DPN) case 10 had antemortem blood collected 5 minutes before death and had a diphenhydramine concentration of $0.11 \mathrm{mg} / \mathrm{L}$. As a result, when using Equation 7 the expected concentration range of diphenhydramine at the time of death would have been between $0.10-0.11 \mathrm{mg} / \mathrm{L}$. At autopsy, heart and mixed blood samples were collected and submitted for analyses (PMI $=24$ hours). Heart blood (HTBL) had a concentration of $0.28 \mathrm{mg} / \mathrm{L}$, while the mixed (MXBL) sample had a concentration of $0.73 \mathrm{mg} / \mathrm{L}$. Both PM concentrations exceeded the calculated range of the expected concentration at the time of death. The PM:AM ratios for HTBL and MXBL were 2.5 and 6.6 , respectively. Statistical analysis of the two PM concentrations determined that the HTBL and MXBL amounts were different. The term mixed blood is a non-descript way of indicating a mixture of blood collected from various regions of the body (potentially both central and peripheral). Trying to determine the significance of the increased concentration obtained in the MXBL, as compared to the other two samples cannot be accurately evaluated. Additionally, the C:P ratio calculation would not be appropriate for this case. What can be shown was that regardless of the origin of the source, both PM samples had concentrations that were higher than expected and they each had PM:AM ratios greater than 1.0. This information suggested that PMR did take place.

The antemortem blood for DPN 03 was collected 10 minutes before death. While diphenhydramine was detected in the AM, the amount was below the LOQ. As a result, 
Table 15: One-Way ANOVA Summary Results for Diphenhydramine Controls $(0.5 \mathrm{mg} / \mathrm{L})$

$(n=41)$

\begin{tabular}{|ccccc|}
\hline Groups & Count & Sum & Average & Variance \\
\hline lot 1 & 15 & 8.62 & 0.57 & 0.013 \\
\hline $\operatorname{lot} \mathbf{2}$ & 19 & 10.50 & 0.55 & 0.002 \\
\hline $\operatorname{lot} \mathbf{3}$ & 7 & 3.46 & 0.49 & 0.002 \\
\hline
\end{tabular}

Table 16: One-Way ANOVA Results for Diphenhydramine Controls 95\% Confidence Limit

\begin{tabular}{|cccccc|}
\hline $\begin{array}{c}\text { Source of } \\
\text { Variation }\end{array}$ & Sum of Squares & df & Mean Square & F-test value & F crit \\
\hline Between lots & 0.03 & 2 & 0.015 & 2.39 & 3.24 \\
\hline
\end{tabular}

$>$ If $\mathrm{F}>\mathrm{F}$ critical, then results are significantly different

$>$ If $\mathrm{F}<\mathrm{F}$ critical, then results are similar 
it would also be expected that the PM concentrations would be below the LOQ as well. Iliac vein blood was submitted in both gray and red-top tubes. Each had a diphenhydramine concentration of $0.1 \mathrm{mg} / \mathrm{L}$. While this value was just at the LLOQ, it did show increased blood concentration after death.

The antemortem blood for DPN 08 was collected four hours before death and had a concentration of $0.15 \mathrm{mg} / \mathrm{L}$. As a result, the expected concentration at the time of death would have been between $0.06-0.12 \mathrm{mg} / \mathrm{L}$. Upon autopsy aorta and iliac vein bloods were submitted for postmortem analyses. Aorta blood had a concentration of $0.48 \mathrm{mg} / \mathrm{L}$, while iliac vein blood had a concentration of $0.57 \mathrm{mg} / \mathrm{L}$. Both PM samples exceeded the expected concentration range. In this case, the amount of diphenhydramine in the peripheral blood exceeded that of the central sourced blood. However, the concentrations for both PM sources fell within the acceptable limits of variation ( $\pm 15 \%)$ established for diphenhydramine. As a result, the concentrations were considered to be statistically similar.

Ratio calculations for DPN 08 rendered C: P results equaling 0.84, while PM: AM ratios were 3.2 and 3.8 for AOBL and ILBL, respectively. The C:P ratio indicated a lack of PMR, while both PM:AM ratios suggested the presence of PMR. An obvious discrepancy between the ratio calculations existed. However, the combination of the PM:AM data as well as the fact that both PM sources had concentrations that were at least four times higher than the expected concentration range, was used by the researcher to determine that PMR was a factor.

Nine and a half hours passed between the AM sample collection and death in case DPN 01. Diphenhydramine was detected in the antemortem, but was at an amount below 
Table 17: Diphenhydramine antemortem samples collected within two elimination half-lives

$$
\begin{gathered}
\mathrm{t}_{1 / 2}=3-14 \text { hours } \\
\mathrm{V}_{\mathrm{d}}=3-4 \mathrm{~L} / \mathrm{kg} \\
\mathrm{CV}=15.0 \%
\end{gathered}
$$

\begin{tabular}{|c|c|c|c|c|c|c|c|c|c|c|}
\hline $\begin{array}{l}\text { AM- } \\
\text { Death }\end{array}$ & Case \# & $\begin{array}{c}\text { Source } \\
\text { (Whole Blood) }\end{array}$ & $\begin{array}{c}\text { Concentration } \\
(\mathrm{mg} / \mathrm{L})\end{array}$ & $\begin{array}{c}\text { AM } \\
\begin{array}{c}\text { Concentration } \\
(\mathrm{mg} / \mathrm{L})\end{array} \\
\end{array}$ & Specimen & $\begin{array}{l}\text { PM: } \\
\text { AM }\end{array}$ & $C: P$ & $\begin{array}{c}\text { PMI } \\
\text { (hours) }\end{array}$ & Race/Sex & Age \\
\hline \multirow{2}{*}{$5 \mathrm{~min}$} & \multirow{2}{*}{$\begin{array}{c}\text { DPN } \\
10 \\
\end{array}$} & Heart & 0.28 & \multirow{2}{*}{0.11} & \multirow{2}{*}{ AMBL } & 2.5 & \multirow{2}{*}{-} & \multirow{2}{*}{24} & \multirow{2}{*}{ WM } & \multirow{2}{*}{61} \\
\hline & & Mix & 0.73 & & & 6.6 & & & & \\
\hline $10 \mathrm{~min}$ & $\begin{array}{c}\text { DPN } \\
03\end{array}$ & Iliac & 0.1 & $<\mathrm{LOQ}$ & AMBL & - & - & 28.5 & WM & 44 \\
\hline \multirow{2}{*}{$\begin{array}{c}4 \\
\text { hours }\end{array}$} & \multirow{2}{*}{$\begin{array}{c}\text { DPN } \\
08\end{array}$} & Aorta & 0.48 & \multirow{2}{*}{0.15} & \multirow{2}{*}{ AMBL } & 3.2 & \multirow{2}{*}{0.84} & \multirow{2}{*}{19} & \multirow[b]{2}{*}{ WF } & \multirow{2}{*}{49} \\
\hline & & Iliac & 0.57 & & & 3.8 & & & & \\
\hline \multirow{2}{*}{$\begin{array}{c}9.5 \\
\text { hours }\end{array}$} & \multirow{2}{*}{$\begin{array}{c}\text { DPN } \\
01\end{array}$} & \multirow{2}{*}{ Mix* } & \multirow{2}{*}{0.12} & \multirow{2}{*}{$<\mathrm{LOQ}$} & \multirow{2}{*}{ AMBL } & - & \multirow{2}{*}{ - } & \multirow{2}{*}{23} & \multirow{2}{*}{ BM } & \multirow{2}{*}{57} \\
\hline & & & & & & - & & & & \\
\hline \multirow{2}{*}{$\begin{array}{c}10 \\
\text { hours }\end{array}$} & \multirow{2}{*}{$\begin{array}{c}\text { DPN } \\
05 \\
\end{array}$} & Iliac & 0.20 & \multirow{2}{*}{0.18} & \multirow{2}{*}{ AMBL } & 1.1 & \multirow{2}{*}{-} & \multirow{2}{*}{11} & \multirow{2}{*}{$\mathrm{BM}$} & \multirow{2}{*}{43} \\
\hline & & Iliac & 0.14 & & & 0.78 & & & & \\
\hline \multirow{2}{*}{$\begin{array}{c}11 \\
\text { hours }\end{array}$} & \multirow{2}{*}{$\begin{array}{c}\text { DPN } \\
06\end{array}$} & Heart & 2.96 & $<\mathrm{I} O \mathrm{O}$ & $\mathrm{AMBI}$ & - & 417 & & & \\
\hline & & Iliac & 0.71 & $<\mathrm{LUQ}$ & AMIBL & - & 4.17 & 25 & $\mathrm{BM}$ & 36 \\
\hline 14 & DPN & Mix & 0.12 & & & 1.2 & & & & 49 \\
\hline hours & 04 & Iliac & 0.11 & 0.10 & AMBL & 1.1 & - & 19 & $\mathrm{WF}$ & 49 \\
\hline 14 & DPN & Heart & 0.53 & Q 27 & & 2.0 & 003 & 17 & & 65 \\
\hline hours & 07 & Iliac & 0.57 & 0.21 & AIVIBL & 2.1 & 0.93 & 17 & WF & 03 \\
\hline
\end{tabular}

Linear range: $0.1-1.0 \mathrm{mg} / \mathrm{L}$

${ }^{1}$ Pulmonary Artery

* Average concentration 
the LOQ $(0.1 \mathrm{mg} / \mathrm{L})$. Postmortem mixed blood was collected in both the red and graytop tubes. The red-top MXBL sample had $0.11 \mathrm{mg} / \mathrm{L}$ of diphenhydramine and the graytop MXBL had $0.13 \mathrm{mg} / \mathrm{L}$. As would be expected for PM samples of the same source, their concentrations were determined to be statistically similar. Additionally, since they were of the same source and of similar concentrations, their results were averaged $(0.12 \mathrm{mg} / \mathrm{L})$. While the exact amount in the AM sample is unknown, it would be expected that during the 9.5 hours between AM sample collection and death, that the amount of diphenhydramine would have continued to decrease, rendering the PM concentration below the LOQ as well. However, that was not the case. Instead, during the 23 hour PMI, the amount of diphenhydramine increased to just within the quantifiable limits of the assay. The apparent increase of drug in the PM sample was indicative of PMR.

Antemortem blood for diphenhydramine 05 was collected ten hours before death and had a concentration of $0.18 \mathrm{mg} / \mathrm{L}$. The expected concentration range at the time of death would have been between $0.02-0.11 \mathrm{mg} / \mathrm{L}$. Iliac vein blood was submitted for $\mathrm{PM}$ analyses in both red and gray-top sampling tubes $(\mathrm{PMI}=11$ hours $)$. The red-top tube had a concentration of $0.20 \mathrm{mg} / \mathrm{L}$ and the gray-top tube had $0.14 \mathrm{mg} / \mathrm{L}$. These values resulted in PM: AM ratios of 1.1 and 0.78 for the RT and GT, respectively. While one PM sample indicated an increase in drug concentration (relative to the antemortem sample), the other PM specimen did not. Statistical evaluation of the PM samples determined that their concentrations were statistically dis-similar. While it is expected that samples collected from the same source would have virtually identical concentrations, this is not always the case. Since the amount of iliac blood is relatively limited, it would not be uncommon for the iliac blood to have been collected from more 
than one site along the vein, or from both veins. In either instance, a concentration discrepancy between tubes can arise. Despite the overall dis-similarity between the tubes, both iliac blood concentrations exceeded the expected concentration range at the time of death. Therefore, it is likely that a small amount of PMR occurred in this case.

In diphenhydramine case 06 , the antemortem was collected 11 hours before death. The antemortem sample had a diphenhydramine concentration below the LOQ. After autopsy, heart and iliac vein bloods were submitted (PMI $=25$ hours). Heart blood had a concentration of $2.96 \mathrm{mg} / \mathrm{L}$, while iliac vein blood had a concentration of $0.71 \mathrm{mg} / \mathrm{L}$. While both the central and peripheral blood sources showed increased concentrations over the AMBL, the central sourced blood was more than four times greater than both the peripheral and antemortem bloods when considering the LOQ. The resulting C:P ratio was 4.17. The increased PM blood concentrations and the high central to peripheral ratio indicated that PMR was a factor in this case. Additionally, the largely elevated heart blood concentration signifies that, diphenhydramine was largely stored in the tissues of the chest cavity and/ or liver and subsequently released during the 25 hour PMI.

The antemortem blood for DPN case 04 had a concentration of $0.10 \mathrm{mg} / \mathrm{L}$ and was collected 14 hours before death. The low concentration and the relatively long AMD interval caused the expected diphenhydramine concentration range $(0.003-0.05 \mathrm{mg} / \mathrm{L})$ to be less than the LOQ. Upon autopsy, mixed and iliac vein bloods were submitted for postmortem analyses. The mixed blood had $0.12 \mathrm{mg} / \mathrm{L}$ of diphenhydramine, while the iliac vein blood had $0.11 \mathrm{mg} / \mathrm{L}$. Postmortem: antemortem ratios equaled 1.2 and 1.1 for MXBL and ILBL, respectively. Both postmortem samples were at least double the upper 
limit of the expected concentration range. This was indicative of diphenhydramine being released and redistributed postmortem, during the 19 hour PMI.

Antemortem for DPN 07 was collected 14 hours before death. The AM sample had $0.27 \mathrm{mg} / \mathrm{L}$ of diphenhydramine. Heart and iliac vein bloods were collected during autopsy. Heart blood had a concentration of $0.53 \mathrm{mg} / \mathrm{L}$ and iliac vein blood had $0.57 \mathrm{mg} / \mathrm{L}$. The expected concentration range at the time of death was between 0.01 and $0.13 \mathrm{mg} / \mathrm{L}$. Both PM samples had amounts that exceeded this range, resulting in PM:AM ratios of 1.96 and 2.11 for HTBL and ILBL, respectively. These values were indicative of PMR. However, the peripheral blood sample exceeded the concentration of the central blood sample, resulting in a C:P ratio of 0.93 , which would indicate a lack of PMR. While the ILBL did have a higher concentration than the HTBL, the difference between the two values was not statistically significant. The PM:AM ratios, as well as the increased levels of diphenhydramine in the PM samples lead the researcher to conclude that PMR did occur.

Seventy-four percent of cases whose AM was collected within two elimination half-lives before death had postmortem concentrations that were statistically greater than their respective AM concentrations. One-hundred percent of the data had PM concentrations greater than their respective calculated concentration range at the time of death. As a result, the overwhelming majority of the first half of the diphenhydramine data returned results that were consistent with PMR. The average $V_{d}$ for diphenhydramine is $3.5 \mathrm{~L} / \mathrm{kg}$ (range $=3-4 \mathrm{~L} / \mathrm{kg}$ ). Any apparent volume of distribution greater than $1 \mathrm{~L} / \mathrm{kg}$ is said to be reflective of a drugs ability to redistribute. In the case of diphenhydramine (who's apparent $V_{d}$ ranges from 3 to 4 times greater than the critical 
limit) the propensity for redistribution is great. A few of the cases had mixed blood submitted as a PM sample. While the exact location of the sample procurement cannot be resolved, these samples give a generalized view of what is occurring within the body after death. Changes in drug concentrations can be identified in mixed samples, just as they are in 'pure' samples. In the absence of the latter, a comparison between a mixed and AM sample can still give important information about the tendency of a drug.

As demonstrated in other chapters of this manuscript, PM:AM ratios and C:P ratios calculated for the same case do not always agree. This was exhibited here in DPN 08 and 07, where both PM:AM ratios were greater than 1.0, but their C:P values were less than 1.0. In the absence of an AM sample, it would have been incorrectly determined that PMR was not present. Perhaps it would be best to view the C:P ratio as proof of PMR occurring as predicted (e.g. more extensively in the central cavity), rather than actual evidence of PMR existing at all.

Also observed within these cases was a discrepancy between two postmortem samples of the same source. Diphenhydramine case 05 had a relatively short PMI of 11 hours. Upon collection, iliac vein blood was stored in both the red and gray-top sample tubes. However, the diphenhydramine drug concentration varied significantly between the two tubes. This case serves as a reminder that assumptions made about sample homogeneity may not always be appropriate.

The second half of this section focuses on the diphenhydramine cases that had antemortem samples collected beyond two elimination half-lives (Table 18) before death. Only two cases fell into this category. While two cases may not be enough data to draw 
any formal conclusions, the addition of these cases to the totality of the diphenhydramine data can be used to reinforce some ideals discussed in the earlier part of this chapter.

Diphenhydramine case 02 had antemortem blood collected 48 hours before death. The AM blood had a concentration of $0.33 \mathrm{mg} / \mathrm{L}$. Aorta and iliac vein bloods were submitted for postmortem analyses. The PM concentrations were $0.56 \mathrm{mg} / \mathrm{L}$ and $0.40 \mathrm{mg} / \mathrm{L}$ for AOBL and ILBL, respectively. After 48 hours (approximately four halflives), the expected concentration range would have been $5.0 \times 10^{-6}$ to $0.03 \mathrm{mg} / \mathrm{L}$. Instead, the amount of drug in both PM samples exceeded that of the AM blood and its expected range. The AOBL:AM ratio equaled 1.70, while the ILBL:AM ratio was equal to 1.21. Additionally, the C:P ratio equaled 1.4. A review of the medical records reported the administration of multiple medications during the 48 hour hospitalization, some of which included naloxone (an opioid antagonist), epinephrine and atropine; however, diphenhydramine administration was not reported. Therefore, there was nothing to suggest that the concentration of the drug would have increased as a result of additional dosing or incomplete distribution. Consequently, it appears that the drug was released and redistributed during the 13 hour PMI.

Statistical analyses of the data collected on DPN 02 showed that the aorta blood sample concentration was significantly different from that of the iliac vein blood. Additionally, when comparing the PM to the AM concentrations, the aorta blood was also significantly different from the antemortem blood concentration. Conversely, the concentration obtained for the iliac vein blood was determined to be similar to the antemortem sample. The comparisons of the PM and AM samples seemed to indicate that while both PM 
Table 18: Diphenhydramine antemortem samples collected after two elimination half-lives

$$
\begin{gathered}
\mathrm{t}_{1 / 2}=3-14 \text { hours } \\
\mathrm{V}_{\mathrm{d}}=3-4 \mathrm{~L} / \mathrm{kg} \\
\mathrm{CV}=15.0 \%
\end{gathered}
$$

\begin{tabular}{|c|c|c|c|c|c|c|c|c|c|c|}
\hline $\begin{array}{c}\text { AM - } \\
\text { Death } \\
\text { (hours) }\end{array}$ & Case \# & $\begin{array}{c}\text { Source } \\
\text { (whole blood) }\end{array}$ & $\begin{array}{c}\text { Concentration } \\
(\mathrm{mg} / \mathrm{L})\end{array}$ & $\begin{array}{c}\mathbf{A M} \\
\text { concentration } \\
(\mathrm{mg} / \mathrm{L})\end{array}$ & Specimen & PM: AM & C: $\mathbf{P}$ & $\begin{array}{c}\text { PMI } \\
\text { (hours) }\end{array}$ & Race/ Sex & Age \\
\hline 20 & $\begin{array}{c}\text { DPN } \\
09\end{array}$ & Mixed* Heart & 0.96 & $<\mathrm{LOQ}$ & AMBL & - & - & 21 & $\mathrm{BF}$ & 65 \\
\hline \multirow{2}{*}{48} & DPN & Aorta & 0.56 & \multirow{2}{*}{0.33} & \multirow{2}{*}{ AMBL } & 1.7 & \multirow{2}{*}{1.4} & \multirow{2}{*}{13} & \multirow{2}{*}{ WF } & \multirow{2}{*}{59} \\
\hline & 02 & Iliac & 0.40 & & & 1.2 & & & & \\
\hline
\end{tabular}

Linear range: $0.1-1.0 \mathrm{mg} / \mathrm{L}$

* Average concentration 
specimens were influenced by redistribution, the aorta blood was affected more extensively.

Diphenhydramine case 09 had an antemortem concentration lower than the LOQ of the assay. Mixed heart blood was submitted in both red-top and gray-top tubes. The RT MXHT blood had a concentration of $0.97 \mathrm{mg} / \mathrm{L}$, while the GT had $0.96 \mathrm{mg} / \mathrm{L}$. Since the PM samples were of the same source and had statistically similar concentrations, the PM results were averaged to equal $0.96 \mathrm{mg} / \mathrm{L}$. The AMBL concentration was below the LOQ; therefore, it can be inferred that the PM concentration was at least $90 \%$ higher than the AM. In this case the AM blood was collected approximately 20 hours before death. There was no indication in the medical records that re-administration was a factor. As a result, it appears as though during the 21 hour PMI, PMR likely occurred.

When reviewing all of the cases as a whole, it became apparent that postmortem redistribution was a real factor when trying to interpret PM blood drug concentrations of diphenhydramine. The data discussed here seemed to suggest that on average at least a 50\% increase in PM concentrations (when compared to AM results) could be expected. While it is generally accepted that blood concentrations obtained from the central cavity have higher concentrations (because of increased sources of possible contamination), this is not necessarily the case for diphenhydramine.

Of all the cases discussed, only four were submitted with both central and peripheral blood for comparison (Chart 6). Two out of the four had statistically higher central concentrations than peripheral. The other two cases (DPN 08 and 07) had central and peripheral data that gave statistically similar results. Admittedly, the amount of central vs. peripheral data was limited. However, it did seem to reinforce the idea that 
redistribution may not always follow the expected pattern, which assumes that quantitative values obtained from peripheral sourced blood are more accurate than central blood. To the contrary, this data would seem to indicate that central and peripheral concentrations may not differ that significantly from each other. Again, reiterating the idea that using the $\mathrm{C}: \mathrm{P}$ ratio as an indicator for PMR may not be the most reliable predictor.

Diphenhydramine 02 had one of the shortest PMI (13 hours) of the cases presented. Despite this, it not only produced data that was indicative of PMR, but it also demonstrated that central and peripheral results could vary significantly in a short amount of time. This would suggest that PMR may begin rather quickly after death.

Finally, for each case discussed, the expected drug concentration range at the time of death was calculated (Equation 7). Chart 7 displays the peripheral blood concentrations as they compare to that of the expected range, denoted by the symbol "[." Diphenhydramine cases 03 and 06 had AM concentrations that were below the LOQ; therefore a quantitative range could not be determined. However if PMR did not occur, the PM concentrations would have been expected to be below the LOQ as well.

Diphenhydramine 05 was submitted with two peripheral sources, the results of both tubes are displayed. When examining the Chart 7 data as a whole, $100 \%$ of the cases exceeded their calculated ranges. Therefore, while a peripheral sourced blood may be the preferred postmortem specimen for quantitative analyses, in the case of diphenhydramine, it likely will not be reflective of the blood drug concentration around the time of death. 
Chart 6: Diphenhydramine Central versus Peripheral Data Error rate: $15 \%$

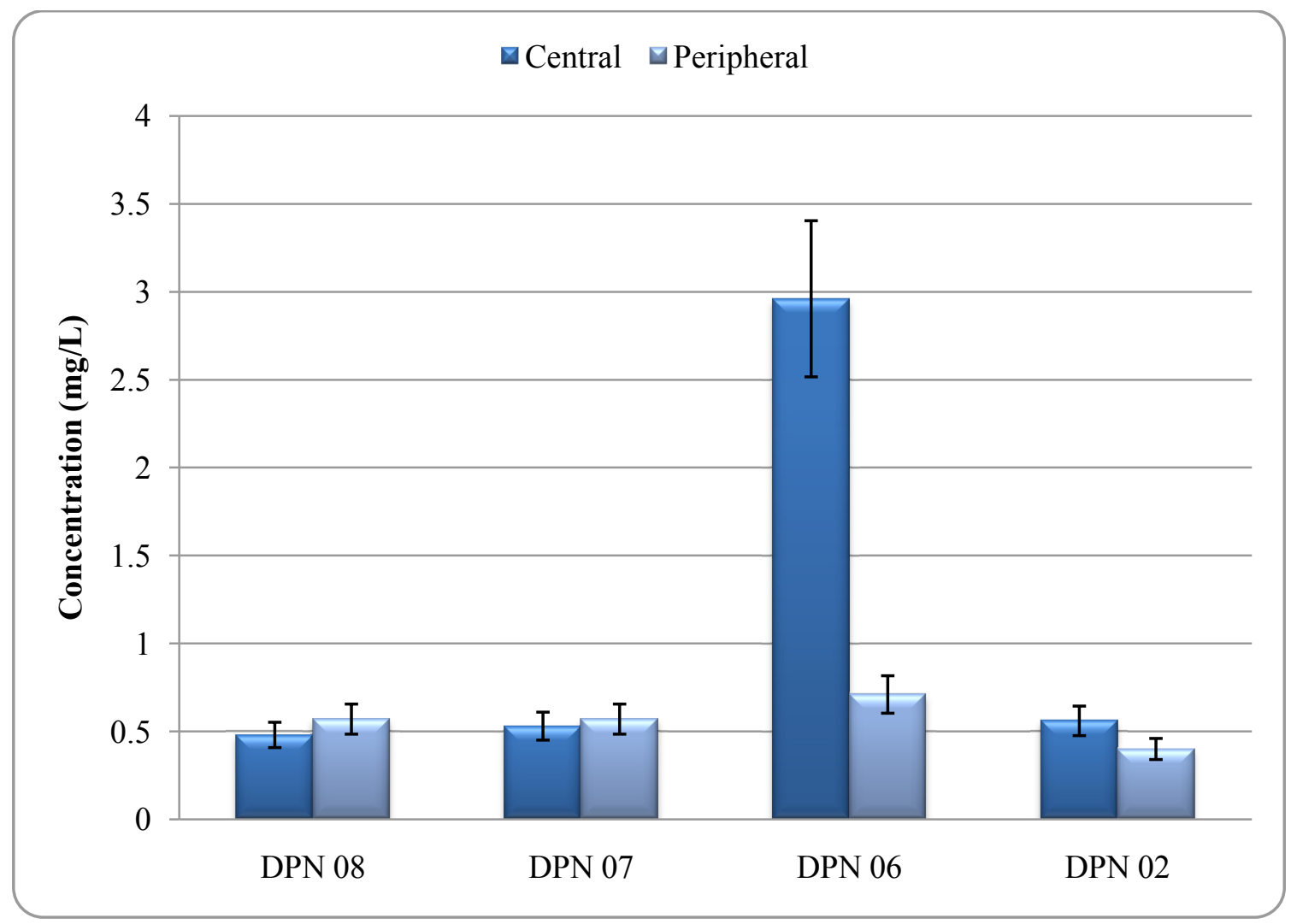




\section{Chart 7: Diphenhydramine Peripheral Source Data}

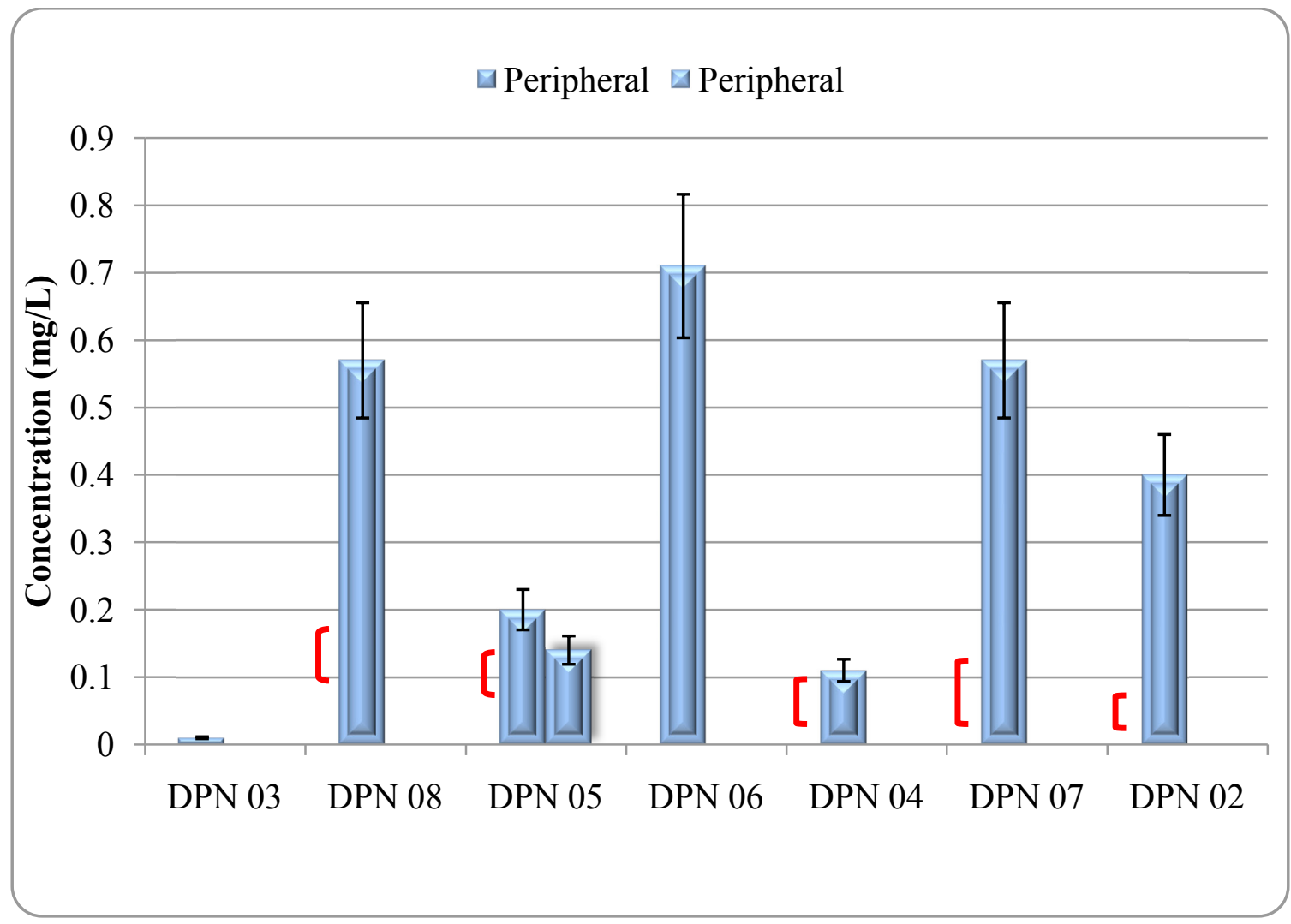

Comparison of diphenhydramine peripheral source blood to its expected drug concentration at the time of death 


\subsection{Benzodiazepines}

Benzodiazepines are a class of drugs with a wide array of uses, including muscle relaxation, sedation and anxiety relief. The broad application of benzodiazepines has helped to make them among the most widely prescribed medications in the world. This study examined diazepam, nordiazepam, alprazolam, midazolam, clonazepam and 7aminoclonazepam specimens in whole blood and serum. As described in section 2.2, the samples were analyzed by GC/ECD (linear range: $0.01-0.2 \mathrm{mg} / \mathrm{L}$ ), HPLC/MS/MS (linear range: $0.01-1.0 \mathrm{mg} / \mathrm{L}$ ) or by HPLC/DAD (linear range: $0.1-10 \mathrm{mg} / \mathrm{L}$ ). Identification of the instrumental analysis is addressed in each of the following sections on a case-by-case basis.

\subsubsection{Alprazolam}

Alprazolam analyses were conducted on two instruments. All data collected as part of routine laboratory analyses were performed on a gas chromatograph/ electron capture detector (GC/ECD). This same instrument was used for research purposes until March 2008. Since that time, the quantitative benzodiazepine research was analyzed by high performance liquid chromatography/ tandem mass spectrometry (HPLC/MS/MS) (refer to section 4.2.2 (b)). The switch from GC/ECD to HPLC/MS/MS allowed for an extended linear concentration range. In addition, the HPLC/MS/MS method monitored seven analytes, while the GC/ECD only quantified three.

The control data produced by both the GC/ECD and the HPLC/MS were statistically analyzed by using a two-way ANOVA $(\alpha=0.05)$. The purpose of the two- 
way ANOVA was to determine if the control data collected from the two systems significantly varied. The F-test result for that comparison was 0.33 , while the $\mathrm{F}_{\text {crit }}$ value equaled 4.4. Since $\mathrm{F}<\mathrm{F}_{\text {crit }}$ the ANOVA results determined that no significant variation was observed between the two instruments (Table 19). Additionally, the amounts of day to day variability, as well as the interaction between the sources of variation, were not considered significantly different either. Since it was determined that the GC/ECD and LC/MS control data were statistically similar, the data generated from both systems were treated as one population. The coefficient of variation for the population equaled $18.73 \%$. Intra-case drug concentrations that were included within the $\pm 18.73 \%$ range were considered similar.

Alprazolam is a benzodiazepine with an average elimination half-life $\left(t_{1 / 2}\right)$ of 11 hours (ranging from $6-27$ hours (57)). It has an apparent volume of distribution $\left(\mathrm{V}_{\mathrm{d}}\right)$ of $0.9-1.3 \mathrm{~L} / \mathrm{kg}$ (57). For this study, 14 cases were evaluated. The data is presented in order of increasing antemortem to death intervals. Those cases whose AM blood was collected within two AMD intervals were included within the first section. Two additional donor cases whose blood was collected within hours of brain death were also included here (Table 20). The second section covers those cases that exceed two elimination half-lives between AM sample collection and death (Table 21). Additionally, one donor case, whose AM blood was collected seven days after death was also included in the second section. 
Table 19: Two-Way Analysis of Variation for Alprazolam Comparison of GC/ECD versus HPLC/MS/MS Controls

\begin{tabular}{|cccccc|}
$\begin{array}{c}\text { Source of } \\
\text { Variation }\end{array}$ & $\begin{array}{c}\text { Sum of } \\
\text { Squares }\end{array}$ & $\boldsymbol{d f}$ & $\begin{array}{c}\text { Mean } \\
\text { Square }\end{array}$ & $\begin{array}{c}\boldsymbol{F} \text {-test } \\
\text { value }\end{array}$ & $\boldsymbol{F}$ crit \\
\hline Day to Day & 0.001191 & 8 & $1.49 \mathrm{E}-04$ & 1.76 & 2.51 \\
\hline $\begin{array}{c}\text { GC/ECD vs. } \\
\text { LC/MS }\end{array}$ & $2.84 \mathrm{E}-05$ & 1 & $2.84 \mathrm{E}-05$ & 0.33 & 4.41 \\
\hline Interaction & 0.000744 & 8 & $9.3 \mathrm{E}-05$ & 1.10 & 2.51 \\
\hline
\end{tabular}

Antemortem blood for Alprazolam (Alp) 09 was collected 13 minutes before death. The AM concentration was $0.09 \mathrm{mg} / \mathrm{L}$. Such a short AM to death time interval would suggest that in the absence of PMR, the postmortem blood concentrations would be similar to that of the AM (ranging from $0.088-0.089 \mathrm{mg} / \mathrm{L}$ ). The postmortem samples submitted in both the red-top (RT) and gray-top (GT) tubes were iliac vein blood samples with a PMI of 21 hours. The RT concentration equaled $0.080 \mathrm{mg} / \mathrm{L}$, with the GT equaling $0.089 \mathrm{mg} / \mathrm{L}$. While, the red-top did render a slightly higher concentration ( $\sim 11.5 \%$ higher), the difference between the two tubes was less than the $18.73 \%$ needed to make the difference statistically significant. Since the PM concentrations were similar and of the same source, their concentrations were averaged to $0.084 \mathrm{mg} / \mathrm{L}$. When using the average, the PM:AM ratio was calculated to equal 0.89 . Additionally, when applying the $\mathrm{CV}$ percentage, it was determined that the $\mathrm{PM}$ and $\mathrm{AM}$ concentrations were statistically similar. The combination of the ratio results, as well as the CV results indicated that PMR did not occur in this case.

Alprazolam 02 AM samples were collected 20 minutes before death and had AM alprazolam concentration of $0.01 \mathrm{mg} / \mathrm{L}$. The expected alprazolam concentration at the time of death would have been between $0.01-0.01 \mathrm{mg} / \mathrm{L}$ ( raw data $=0.0096-$ 
Table 20: Alprazolam antemortem samples collected within two elimination half-lives

$$
t_{1 / 2}=(6-27 \text { hours })
$$

$V_{d}=0.9-1.3 \mathrm{~L} / \mathrm{kg}$

CV population $=18.73 \%$

\begin{tabular}{|c|c|c|c|c|c|c|c|c|c|c|}
\hline $\begin{array}{l}\text { AM - } \\
\text { Death }\end{array}$ & Case \# & $\begin{array}{c}\text { Source } \\
\text { (whole blood) }\end{array}$ & $\begin{array}{c}\text { Concentration } \\
(\mathrm{mg} / \mathrm{L})\end{array}$ & $\begin{array}{c}\mathbf{A M} \\
\begin{array}{c}\text { concentration } \\
(\mathbf{m g} / \mathbf{L})\end{array} \\
\end{array}$ & Specimen & PM: AM & $\mathrm{C}: P$ & $\begin{array}{c}\text { PMI } \\
\text { (hours) }\end{array}$ & Race/Sex & Age \\
\hline $13 \mathrm{~min}$ & $\begin{array}{l}\text { Alp } \\
09^{1}\end{array}$ & Iliac* & 0.084 & 0.09 & AMBL & 0.9 & - & 21 & WM & 21 \\
\hline \multirow{2}{*}{$20 \mathrm{~min}$} & \multirow{2}{*}{$\begin{array}{l}\text { Alp } \\
02^{1}\end{array}$} & Aorta & 0.02 & \multirow{2}{*}{0.01} & \multirow{2}{*}{ AMBL } & 2.0 & \multirow{2}{*}{0.5} & \multirow{2}{*}{45.5} & \multirow{2}{*}{ WM } & \multirow{2}{*}{26} \\
\hline & & Iliac & 0.04 & & & 4.0 & & & & \\
\hline \multirow{2}{*}{$40 \mathrm{~min}$} & \multirow{2}{*}{$\begin{array}{c}\text { Alp } \\
14^{2}\end{array}$} & Chest & 0.04 & \multirow{2}{*}{0.02} & \multirow{2}{*}{ AMBL } & 2.0 & \multirow{2}{*}{1.3} & \multirow{2}{*}{52} & \multirow{2}{*}{ WM } & \multirow{2}{*}{52} \\
\hline & & Iliac & 0.03 & & & 1.5 & & & & \\
\hline \multirow{2}{*}{$1.5 \mathrm{hrs}$} & \multirow{2}{*}{$\begin{array}{c}\text { Alp } \\
06^{1}\end{array}$} & Chest & $<$ LOQ & \multirow{2}{*}{$<\mathrm{LOQ}$} & \multirow{2}{*}{ AMBL } & - & \multirow{2}{*}{-} & \multirow{2}{*}{19} & \multirow{2}{*}{ WM } & \multirow{2}{*}{40} \\
\hline & & Chest & $<$ LOQ & & & - & & & & \\
\hline \multirow{2}{*}{$11 \mathrm{hrs}$} & \multirow{2}{*}{$\begin{array}{l}\text { Alp } \\
03^{1}\end{array}$} & Heart & NDD & \multirow{2}{*}{0.01} & \multirow{2}{*}{ AM S } & - & \multirow{2}{*}{-} & \multirow{2}{*}{19} & \multirow{2}{*}{ WM } & \multirow{2}{*}{50} \\
\hline & & Iliac & NDD & & & - & & & & \\
\hline \multirow{2}{*}{$12 \mathrm{hrs}$} & \multirow{2}{*}{$\begin{array}{c}\text { Alp } \\
11^{1} \\
\end{array}$} & Aorta & 0.48 & \multirow{2}{*}{$>$ LOQ } & \multirow{2}{*}{ AM S } & - & 11 & 54 & WM & 68 \\
\hline & & Iliac & 0.43 & & & - & 1.1 & 34 & WVIVI & 00 \\
\hline $15 \mathrm{hro}$ & Alp & Heart & 0.04 & 003 & $A M D I$ & 1.3 & 08 & 225 & WM & 41 \\
\hline $15 \mathrm{nrs}$ & $04^{1}$ & Iliac & 0.05 & 0.03 & AIVIDL & 1.7 & 0.0 & 32.5 & WIVI & 41 \\
\hline $30 \mathrm{~min}$ & Alp & Heart & $<$ LOQ & & & - & & & & \\
\hline AFTER & $05^{2}$ & Iliac & $<\mathrm{LOQ}$ & 0.01 & AMBL & - & - & 89 & WM & 45 \\
\hline
\end{tabular}




\begin{tabular}{|c|c|c|c|c|c|c|c|c|c|c|}
\hline $\begin{array}{l}\text { AM - } \\
\text { Death }\end{array}$ & Case \# & $\begin{array}{c}\text { Source } \\
\text { (whole blood) }\end{array}$ & $\begin{array}{c}\text { Concentration } \\
(\mathrm{mg} / \mathrm{L})\end{array}$ & $\begin{array}{c}\text { AM } \\
\text { concentration } \\
(\mathrm{mg} / \mathrm{L})\end{array}$ & Specimen & PM: AM & $\mathrm{C}: \mathrm{P}$ & $\begin{array}{c}\text { PMI } \\
\text { (hours) }\end{array}$ & Race/Sex & Age \\
\hline \multirow{2}{*}{$\begin{array}{c}6 \mathrm{hrs} \\
\text { AFTER }\end{array}$} & \multirow{2}{*}{$\begin{array}{l}\text { Alp } \\
01^{1}\end{array}$} & Heart & NDD & \multirow{2}{*}{0.01} & \multirow{2}{*}{ AMBL } & - & \multirow{2}{*}{-} & \multirow{2}{*}{14} & \multirow{2}{*}{ WM } & \multirow{2}{*}{21} \\
\hline & & Iliac & NDD & & & - & & & & \\
\hline
\end{tabular}

${ }^{1} \mathrm{GC} / \mathrm{ECD}$ data; linear range $=0.01-0.2 \mathrm{mg} / \mathrm{L}$

${ }^{2} \mathrm{LC} / \mathrm{MS} / \mathrm{MS}$ data; linear range $=0.01-1.0 \mathrm{mg} / \mathrm{L}$

* Average concentration

NDD $=$ No Drugs Detected

Table 20, continued 
$0.0099 \mathrm{mg} / \mathrm{L})$. The postmortem aorta and iliac vein blood samples had a PMI of 45.5 hours and concentrations of 0.02 and $0.04 \mathrm{mg} / \mathrm{L}$, respectively. These concentrations significantly exceeded their expected concentration range $(\mathrm{CV} \pm 18.73 \%)$. The central to peripheral ratio equaled 0.5, while the aorta blood:AM blood ratio equaled 2.0 and the iliac vein blood: AM blood equaled 4.0. Historically it is believed that when central:peripheral and/ or PM:AM ratios are greater than or equal to 1.0, PMR likely occurred. However, in this case the $\mathrm{C}: \mathrm{P}<1.0$, but both PM: AM ratios were $>1.0$. When viewing the Alp 02 data as a whole, both PM samples were determined to be statistically different from their AM concentrations. This in combination with the PM:AM ratio data has caused the researcher to determine that PMR was a factor in this case. Additionally, the aorta and iliac vein blood samples were determined to be statistically dis-similar to each other. This was of particular interest because the iliac vein blood concentration was greater than the aorta blood concentration (hence $\mathrm{C}: \mathrm{P}<$ 1.0). While it is generally accepted that PMR is more pronounced in centrally located blood sources, this case would be an exception to that rule.

The deceased in Alp 14 was the victim of a homicide. He was transported to the hospital and had his blood collected 40 minutes before death (AM concentration = $0.02 \mathrm{mg} / \mathrm{L}$ ), making his expected alprazolam concentration at the time of death range between $0.018-0.019 \mathrm{mg} / \mathrm{L}$. Postmortem submission included a sample obtained from the left chest cavity $(0.04 \mathrm{mg} / \mathrm{L})$ and the iliac vein $(0.03 \mathrm{mg} / \mathrm{L})$. Both PM samples had concentrations higher than the AM. However, only the left chest cavity blood was determined to be statistically higher than the AM sample. The CHBL:AM ratio equaled 2.0, while the ILBL:AM ratio equaled 1.5. Additionally, while the CHBL:ILBL ratio 
equaled 1.3 their concentration variation was within the $\pm 18.73 \%$ range. All of the ratio data indicated that postmortem redistribution occurred during the 52 hour postmortem interval, despite the statistical similarities among most of the data. The researcher was not able to find any literature comparing ratio data to statistical significance. Therefore, it may be possible that the two are mutually exclusive. An alternative explanation for the $\mathrm{AM}$ and $\mathrm{PM}$ concentration differences could be that the victim was still absorbing alprazolam at the time of death. If this were the case, the expected concentration at the time of death would have been slightly higher than the AM concentration, causing PM levels to be higher, which may not have been deemed statistically different than the AM concentration (as was the case with the ILBL).

The decedent in Alp 06 was a victim of homicide. His AM blood was collected 1.5 hours before death, but had an alprazolam concentration below the LOQ (GC/ECD limit of quantitation $=0.01 \mathrm{mg} / \mathrm{L}$ ). The postmortem chest blood samples (collected in both a red-top polypropylene and gray-top vacutainer tubes) had a PMI of 19 hours. Both PM samples contained alprazolam at concentrations below the LOQ. While these results were not quantitative, they were still important. The AM blood had alprazolam detected, but at levels below quantitation. Therefore, there was some potential for PMR. However, none could be identified during the 19 hour PMI. This was an example of how a short antemortem to death interval and a relatively short PMI can result in a lack of significant change in concentration between AM and PM samples. 


\section{Equation 8: Alprazolam's blood to plasma ratio (serum concentration $)(0.8) \cong$ blood concentration}

For Alp 03, AM blood was collected in a serum separator tube. As a result, the specimen available for analysis was serum, not whole blood. However, as indicated in Baselt (57), alprazolam's blood to plasma ratio equals 0.8. Consequently, the result of a given serum concentration multiplied by 0.8 would be approximately equal to that of a whole blood concentration (Equation 8). For this case, the AM blood was collected 11 hours before death and had a concentration of $0.01 \mathrm{mg} / \mathrm{L}$. When applying this result to Equation 8 the equivalent blood concentration would be $0.008 \mathrm{mg} / \mathrm{L}$. When rounded the equivalent blood concentration would equal $0.01 \mathrm{mg} / \mathrm{L}$. With an antemortem to death interval of 11 hours, the expected concentration range at the time of death would be 0.003 $-0.007 \mathrm{mg} / \mathrm{L}$. Analysis of the postmortem samples $(\mathrm{PMI}=19$ hours $)$ did not detect the presence of alprazolam. This sample was analyzed by GC/ECD which had a limit of detection of $0.002 \mathrm{mg} / \mathrm{L}$. Although the expected concentration range was low, it would have fallen within the detectable limits of the assay. With no PM concentrations to consider, it can be concluded that PMR was not a factor in this case.

In Alp 11, antemortem serum, as opposed to blood, was collected 12 hours before death. The serum was determined to have an AM concentration greater than the upper limit of quantitation (ULOQ) $(0.2 \mathrm{mg} / \mathrm{L}$; analyzed by GC/ECD). The volume of the sample was not sufficient for subsequent dilution and re-analysis. The original paperwork documented the concentration, although exceeding the validated linear range, at $0.30 \mathrm{mg} / \mathrm{L}$. Postmortem submission $(\mathrm{PMI}=54$ hours $)$ included aorta and iliac vein 
bloods. Since the AM concentration exceeded the linear range, the PM samples were diluted prior to analysis to ensure that their concentrations would fall within the validated range. The aorta and iliac vein blood concentrations were determined to be $0.48 \mathrm{mg} / \mathrm{L}$ and $0.43 \mathrm{mg} / \mathrm{L}$, respectively. Assuming instrument linearity up to $0.30 \mathrm{mg} / \mathrm{L}$, the expected concentration at the time of death would have been between $0.07-0.22 \mathrm{mg} / \mathrm{L}$. When calculating the AOBL:AM, ILBL:AM, as well as the AOBL:ILBL ratios the values were determined to be 1.6, 1.4 and 1.1, respectively. All three ratio values indicated that PMR did occur. Alternatively, it should be considered that with such a large amount of drug in the system, it is possible that the body was still in the absorptive stage of the pharmacokinetic process, during the 12 hours between collection and death. This would have resulted in an increase of drug concentration that could have been detected in the PM sample. Unfortunately, based on the information given there is no way of knowing the exact cause of the increased concentration.

Alprazolam 04 was collected 15 hours before death and had a concentration of $0.03 \mathrm{mg} / \mathrm{L}$. As a result, the expected concentration at the time of death would have ranged between $0.005-0.02 \mathrm{mg} / \mathrm{L}$. The postmortem heart blood concentration was $0.04 \mathrm{mg} / \mathrm{L}$ and postmortem iliac vein blood concentration equaled $0.05 \mathrm{mg} / \mathrm{L}$. Both PM samples (PMI of 32.5 hours) had concentrations higher than that of the AM blood, although only the difference between the iliac blood and AM were determined to be statistically dis-similar. The HTBL:AM and ILBL:AM ratios were 1.3 and 1.7, respectively. Both of these calculations suggested the presence of PMR. Conversely, the heart: iliac vein ratio, which equaled 0.8 , did not. The discrepancy observed in the ratio calculations were of concern as both C:P and PM:AM ratios are supposed to be indicative 
of PMR. Upon reviewing the data, even though the HTBL concentrations were not statistically different from the AM concentration, both PM concentrations greatly exceeded the expected concentration range. Therefore, it was determined that PMR was a factor here.

Alprazolam 05 was the case of an organ donor. His AM samples submitted for testing were drawn 30 minutes after brain death (refer to section 1.6.5). The calculated concentration of alprazolam was at the limit of quantitation (LOQ) $(0.01 \mathrm{mg} / \mathrm{L})$. The decedent in this case was maintained on life support for three days while awaiting organ procurement. Based on the official time of brain death, his PMI would have been 89 hours. However, he was receiving fluids and had functioning organs for an additional three days. Therefore, for the purposes of this experiment, his actual PMI (time between organ procurement and autopsy) was less than 24 hours, which would make his AM to death interval closer to 72 hours. A 72 hour AM to death interval for alprazolam would result in anywhere from three to twelve elimination half-lives. It would be expected that the concentration at the time of death would be very low, if detectable at all. Analysis of the PM heart and iliac vein blood samples were each below the LOQ of the assay. This indicated that no PMR occurred between the 24 hours of organ procurement and autopsy.

Alprazolam 01 was a 21 year old white male who suffered from both depression and anxiety. He would self medicate by obtaining alprazolam (dosage unknown) whenever he felt it necessary. The decedent ultimately committed suicide and became an organ donor upon family approval. His AM blood, collected six hours after brain death, had an alprazolam concentration of $0.01 \mathrm{mg} / \mathrm{L}$. His postmortem samples (heart and iliac vein bloods) were both negative for alprazolam (GC/ECD limit of detection = 
$0.002 \mathrm{mg} / \mathrm{L}$ ). The PMI was 14 hours, the shortest time of all 14 cases tested. Organ donors often have postmortem blood negative of any drug not given in preparation of surgery. This is a direct result of the amount of fluids infused through the body just prior to surgery (in this case at least 6 hours of fluid). Infusions often dilute drugs of toxicological importance to concentrations well below quantitation limits. The detection of alprazolam in the AM and its subsequent absence in the PM suggested that PMR was not a factor in this case.

The majority of the above mentioned cases had AM collection times within two elimination half-lives before death. There were two exceptions, Alp 05 and Alp 01, which were both organ donors. It is well known that drugs of toxicological importance can be lost during the preparatory procedure of organ procurement. However, this brings to light the importance of accurate date and time information concerning AM collection, brain death and organ removal. On the surface, it appeared that the AM collection time was ideal for Alp 05. However, with further investigation it was revealed that the date of the AM was actually collected about three days before procurement. This would indicate that the patient received infusions for an additional 72 hours after blood collection. Even though the presence of alprazolam was not critical in this case, this type of information could influence the interpretation of results from other cases. For example, ALP 01 was also an organ donor. The decedent had a history of psychological issues and intermittent alprazolam usage. His AM was collected six hours after he was declared brain dead. From a postmortem toxicological standpoint, that means AM collection was not until 6 hours after fluid infusion. Although alprazolam did not contribute to the death of either 
individual, in other situations accurate timeline information could be a crucial factor in accurately assessing a cause of death.

When the cases are organized in order of increasing AM to death interval, the first four cases (Alprazolam 09, 02, 14 and 06) were of particular interest. Each case had AM blood collected $\leq 1.5$ hours before death. Alprazolam 09 and 06 had the shortest and longest AM to death interval (of the first four), respectively. They each had relatively short PMI $\unlhd 21$ hours) and both were determined to not be affe cted by PMR. Alprazolam 02 and 14 had AM blood collected within 40 minutes of death. Alprazolam 02 had a PMI of 45.5 hours, while Alp 14 had a PMI of 52 hours. Both of these cases were potentially affected by redistribution. While four cases are not sufficient to make any definitive decisions, it would appear that the PMI can greatly affect PM drug concentrations even when the AM to death collection intervals are relatively short.

Additionally seven cases were submitted with both central and peripheral sources. Of those, four had quantitative data for both sources (Alp 02, 04, 11 and 14). Only Alp 02 had PM source data that was statistically different, all other cases gave similar results. This was of particular interest because toxicologists commonly believe that central sourced blood is more susceptible to redistribution. While two of the four cases did show higher central sourced concentrations; overall, they were not statistically different. The one case that showed the dis-similarity had a higher peripheral blood concentration, indicating that the peripheral sample was more subject to PMR in that case. Therefore, the belief that quantitative data collected from the peripheral source is less likely to be contaminated appears to be questionable. 
In most situations observed in the alprazolam data both C:P, as well as PM:AM ratios were in agreement as to whether or not PMR occurred. However, Alp 02 and 04 both had PM: AM ratios $\geq 1.0$ and $\mathrm{C}: \mathrm{P}$ ratio $<1.0$. Both cases were determined to be affected by PMR; however, because their PM concentrations exceeded their expected concentration ranges. In addition discrepancies between statistical significance levels and ratio calculations also arose. This was demonstrated in alprazolam cases 04 and 14, which were both determined to be potentially affected by PMR. The statistical analysis of Alp 04 and the ILBL of Alp 14 showed concentration similarities between PM and AM results. Since the relationship between statistical significance and PMR is not well understood, all of the data and calculations produced for each case had to be considered in its entirety. Only then could a determination be made as to whether or not redistribution influenced the results.

The remaining alprazolam cases all had antemortem collection times that exceeded two elimination half-lives (Table 21). The first of those was alprazolam 10. The antemortem blood received in the laboratory for Alp 10 was dated one day before death but did not specify a time. When calculating the time between hospital arrival and death, no more than 38.5 hours could have passed between AM collection and death. Routine laboratory testing of the AM sample resulted in a concentration of $0.13 \mathrm{mg} / \mathrm{L}$ for alprazolam. Sometime after the blood draw, the patient was declared brain dead and became an organ donor. Organ procurement began five hours after brain death. Postmortem aorta blood concentrations (submitted in both red and gray tubes) were 0.01 $\mathrm{mg} / \mathrm{L}$ each. As a result, the PM aorta blood concentrations were averaged. All subsequent calculations will be based on the average value of $0.01 \mathrm{mg} / \mathrm{L}$. 
If the AM blood for Alp 10 was in fact collected 38.5 hours before death, then the expected drug concentration range at the time of death would have been between $0.001-$ $0.06 \mathrm{mg} / \mathrm{L}$. The actual PM concentration was within the expected range. This indicates that the infusion of fluids (given in preparation of organ procurement) did not negatively affect the PM drug concentration. Additionally, the aorta:AM ratios equaled 0.08. The data collected for this case did not indicate that PMR occurred.

Alprazolam 08 had an AM concentration of $0.04 \mathrm{mg} / \mathrm{L}$ from a sample collected 45 hours before death. The expected concentration at the time of death ranged from $2.2 \mathrm{x}$ $10^{-4}-0.01 \mathrm{mg} / \mathrm{L}$. The PM iliac vein and heart blood samples had a PMI of 28.5 hours and concentrations of $0.01 \mathrm{mg} / \mathrm{L}$ each. The central:peripheral ratio equaled 1.0, while the heart blood:AM, as well as the ILBL:AM ratios each equaled 0.25. While the C:P ratios indicated the possibility of PMR, the PM:AM ratios did not. It has been determined by the researcher that since the PM concentrations fell within the expected range that PMR did not occur.

In Alp 13, the AM blood sample was collected three days before death. The decedent was transported to the hospital as a result of an intentional drug overdose. The AM alprazolam concentration was $0.26 \mathrm{mg} / \mathrm{L}$, well above therapeutic levels $(0.02-$ $0.06 \mathrm{mg} / \mathrm{L}(57))$. The expected concentration at the time of death ranged from $6.3 \times 10^{-5}-$ $0.04 \mathrm{mg} / \mathrm{L}$. The aorta and iliac vein blood samples, collected 24 hours after death, had statistically similar concentrations of $0.1 \mathrm{mg} / \mathrm{L}$ and $0.08 \mathrm{mg} / \mathrm{L}$, respectively. Hospital records indicated that the decedent presented with overdose symptoms caused by the ingestion of oxycodone and alprazolam. While both PM concentrations exceeded their expected range, the aorta: AM and iliac vein:AM ratios equaled 0.31 and 0.42 , 
Table 21: Alprazolam antemortem samples collected after two elimination half-lives

$$
\begin{gathered}
t_{1 / 2}=(6-27 \text { hours }) \\
V_{d}=0.9-1.3 \mathrm{~L} / \mathrm{kg} \\
C V=18.73 \%
\end{gathered}
$$

\begin{tabular}{|c|c|c|c|c|c|c|c|c|c|c|}
\hline $\begin{array}{l}\text { AM - } \\
\text { Death }\end{array}$ & Case \# & $\begin{array}{c}\text { Source } \\
\text { (whole blood) }\end{array}$ & $\begin{array}{c}\text { Concentration } \\
(\mathrm{mg} / \mathrm{L})\end{array}$ & $\begin{array}{c}\text { AM } \\
\text { concentration } \\
(\mathrm{mg} / \mathrm{L}) \\
\end{array}$ & Specimen & PM:AM & C: P & $\begin{array}{c}\text { PMI } \\
\text { (hours) }\end{array}$ & Race/Sex & Age \\
\hline \multirow{2}{*}{$38.5 \mathrm{hrs}$} & Alp & \multirow{2}{*}{ Aorta* } & \multirow{2}{*}{0.01} & \multirow{2}{*}{0.13} & \multirow{2}{*}{ AMBL } & 0.08 & \multirow[b]{2}{*}{ - } & \multirow{2}{*}{43} & \multirow{2}{*}{ WM } & \multirow{2}{*}{29} \\
\hline & $10^{2}$ & & & & & 0.08 & & & & \\
\hline \multirow{2}{*}{$45 \mathrm{hrs}$} & Alp & Heart & 0.01 & \multirow{2}{*}{0.04} & \multirow{2}{*}{ AMBL } & 0.25 & \multirow{2}{*}{1.0} & \multirow{2}{*}{28.5} & \multirow{2}{*}{ WF } & \multirow[b]{2}{*}{52} \\
\hline & $08^{1}$ & Iliac & 0.01 & & & 0.25 & & & & \\
\hline \multirow{2}{*}{3 days } & Alp & Aorta & 0.08 & \multirow{2}{*}{0.26} & \multirow{2}{*}{ AMBL } & 0.31 & \multirow{2}{*}{0.72} & \multirow{2}{*}{24} & \multirow{2}{*}{ WM } & \multirow{2}{*}{53} \\
\hline & $13^{2}$ & Iliac & 0.11 & & & 0.42 & & & & \\
\hline 5 days & $\begin{array}{l}\text { Alp } \\
07^{1}\end{array}$ & IVC & $<\mathrm{LOQ}$ & 0.02 & AMBL & - & - & 16 & WM & 45 \\
\hline \multirow{2}{*}{$\begin{array}{c}7 \text { days } \\
\text { AFTER }\end{array}$} & Alp & Aorta & 0.30 & \multirow{2}{*}{0.9} & \multirow{2}{*}{$\mathrm{AMBL}^{3}$} & 0.33 & \multirow{2}{*}{1.8} & \multirow{2}{*}{36} & \multirow{2}{*}{ WM } & \multirow{2}{*}{71} \\
\hline & $12^{2}$ & Iliac & 0.17 & & & 0.19 & & & & \\
\hline
\end{tabular}

${ }^{1} \mathrm{GC} / \mathrm{ECD}$ data; linear range $=0.01-0.2 \mathrm{mg} / \mathrm{L}$

${ }^{2} \mathrm{LC} / \mathrm{MS} / \mathrm{MS}$ data; linear range $=0.01-1.0 \mathrm{mg} / \mathrm{L}$

${ }^{3}$ Not collected during the terminal event

* Average concentration 
respectively. Additionally, the central:peripheral ratio equaled 0.73 . All of the ratio data indicated a lack of PMR; however, the PM concentrations exceeded their expected range. At the time of death, the body's ability to metabolize and eliminate drugs from the system could have been compromised because of the overdose. As a result, although (based on the concentrations) it looks as if PMR occurred, the role of the overdose cannot be overlooked. Consequently, it cannot be determined if PMR was a factor.

The antemortem blood for Alp 07 was collected 5 days before death and had a concentration of $0.02 \mathrm{mg} / \mathrm{L}$. Medical records did not indicate the administration of alprazolam during the decedent's five day hospitalization, which would suggest that the drug was ingested before his admittance. The combination of the low AM blood concentration and the five day AMD interval resulted in an expected concentration range at the time of death being at levels below both the LOD and the LOQ (expected range $=$ $1.9 \times 10^{-8}-9.2 \times 10^{-4} \mathrm{mg} / \mathrm{L}$ ). An autopsy was conducted 16 hours after death, and inferior vena cava (IVC) blood was submitted for toxicological analyses. Alprazolam was detected in the IVC BL but at a concentration below the LOQ $(0.01 \mathrm{mg} / \mathrm{L})$. The PM blood concentration was below that of the AM, indicating that metabolism did occur. However the fact that alprazolam was detected in the PM sample, suggests that its concentration greatly exceeded the expected concentration range.

The decedent in Alp 12 intentionally overdosed on his alprazolam prescription and phoned family members to make them aware. He was transported by emergency services to the hospital, treated and survived. However, he remained an inpatient for approximately 3.5 weeks. The patient exhibited anxious and aggressive behavior and as a result was prescribed $0.5 \mathrm{mg}$ tablets of alprazolam twice daily and an additional $1 \mathrm{mg}$ 
tablet at bed time. On the date of his death, he phoned family members to inform them that he had taken 40 alprazolam tablets, while still an in-patient. He was treated, but ultimately died approximately 2.5 hours later.

The AM blood submitted for analysis was dated the day of his hospital admission (approximately 3.5 weeks before death). This sample had a concentration of $0.9 \mathrm{mg} / \mathrm{L}$, which would be indicative of an overdose. However, he was treated and stabilized after this blood draw. Any AM blood samples that may have been collected during the terminal event were not submitted with the original AM sample to the MDME Department. Thirty-six hours after death, aorta and iliac vein blood samples were collected. Upon analysis, the aorta blood concentration was $0.30 \mathrm{mg} / \mathrm{L}$, while the iliac blood was almost half that at $0.17 \mathrm{mg} / \mathrm{L}$. Since the AM sample analyzed did not correlate with the terminal event, its concentration cannot be further considered. However, when examining the postmortem samples, the AOBL:ILBL ratio equaled 1.8. Additionally, the aorta and iliac vein blood sample concentrations were determined to be statistically dissimilar. In the absence of the appropriate AM sample, it cannot be determined if PMR was a factor for this case. In all likelihood, a relevant AM sample would have been collected during the absorptive phase. As a result any discrepancy between AM and PM concentrations could have been a result of incomplete distribution, as opposed to PMR.

The alprazolam cases discussed in the second half of this section bring to light some important considerations. When AM samples are collected more than two elimination half-lives before death, it becomes increasingly important to have accurate and reliable records (including both social and medical histories), which include a 
timeline leading up to the terminal event. Increased time intervals may, at times, equate to increased confounding circumstances, as was the case in Alp 12.

Alprazolam 12 was an example of how important it is to view all of the information as a whole. If the AM sample had been the only sample tested, then the cause of death would have been due to drug toxicity. While ultimately, that would have been accurate, that particular AM sample was not directly related to the actual terminal event. Had the decedent not overdosed the second time and died from causes unrelated to alprazolam, the cause of death would have been inaccurately assigned. This is why social and medical histories, in combination with toxicological findings can all impact the death investigative process.

Alprazolam cases 08,10 and 13 were useful as well. Alprazolam 08 and 10 were examples of how a drug can be present and not have an influence on the cause of death. In contrast, in cases of overdose the overwhelming presence of a drug may hinder the body's ability to eliminate the drug. Alprazolam 13 is an example of the fact that a person can live for three days after an overdose and still maintain a level of drug that exceeds therapeutic levels. In their entirety, the alprazolam cases show that depending on any one factor (or piece of information) to determine the cause of death can lead to inaccuracies. Therefore it is important to consider all the facts before drawing any formal conclusions.

Finally, it was also important to combine the two alprazolam sections and examine the intra-case dynamics. The relationship between central and peripheral results, as well as how well peripheral concentrations compare to their expected concentration ranges was examined. Of the fourteen cases discussed, ten were submitted 
with both central and peripheral sourced blood (Chart 8). Out of those ten cases, six had quantifiable postmortem results. Through statistical analyses, it was determined that in $83 \%$ of the cases no significant difference existed between the two sources. Although peripheral blood is the preferred source for postmortem analyses, this data suggested that in the majority of instances no measurable difference existed between the two anatomical locations. The peripheral data was further evaluated in Chart 9. In order to better interpret the meaning of the postmortem results, each case that had quantifiable AM values was subjected to Equation 7 to determine the expected concentration range at the time of death. This range was an important factor used throughout the chapter to help determine which cases were affected by PMR. In Chart 9 the peripheral results were compared to the expected concentration ranges for each case. The symbol "[" was used to denote the concentration range. At times, the rounded results of the upper and lower ends of the range were equal. For those cases the symbol "-" was used. Alprazolam case 03 had quantifiable amounts of the drug in the AM sample $(0.01 \mathrm{mg} / \mathrm{L})$; however it was not detected in the PM sample. Consequently, the case was represented with the expected range symbol "[", despite the absence of a peripheral result. In $50 \%$ of the alprazolam cases, the peripheral blood concentrations were higher than what would have been expected at the time of death. Twenty-five percent of the data was below the expected range, and the remaining $25 \%$ was within range. The data produced here seemed to indicate that it would not be likely that PM peripheral blood results would accurately reflect the blood drug concentration of a person around the time of death.

Furthermore, when reviewing all 14 cases, regardless of anatomical location, only $21 \%$ of them had data consistent with PMR. Fifty percent of the cases showed a lack of 


\section{Chart 8: Alprazolam Central versus Peripheral Data}

Error rate $=19 \%$

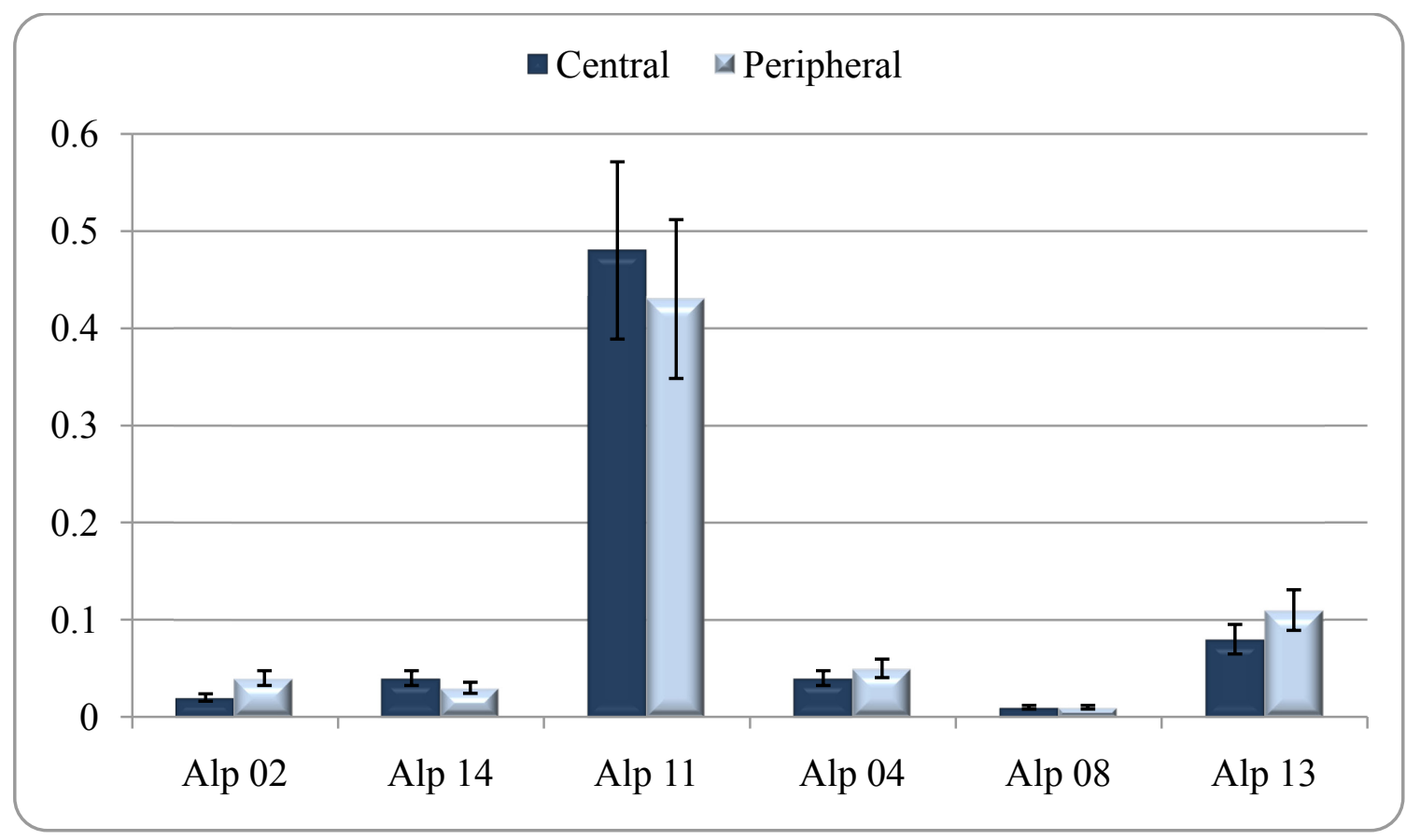




\section{Chart 9: Peripheral Alprazolam Results}

Error rate $=19 \%$

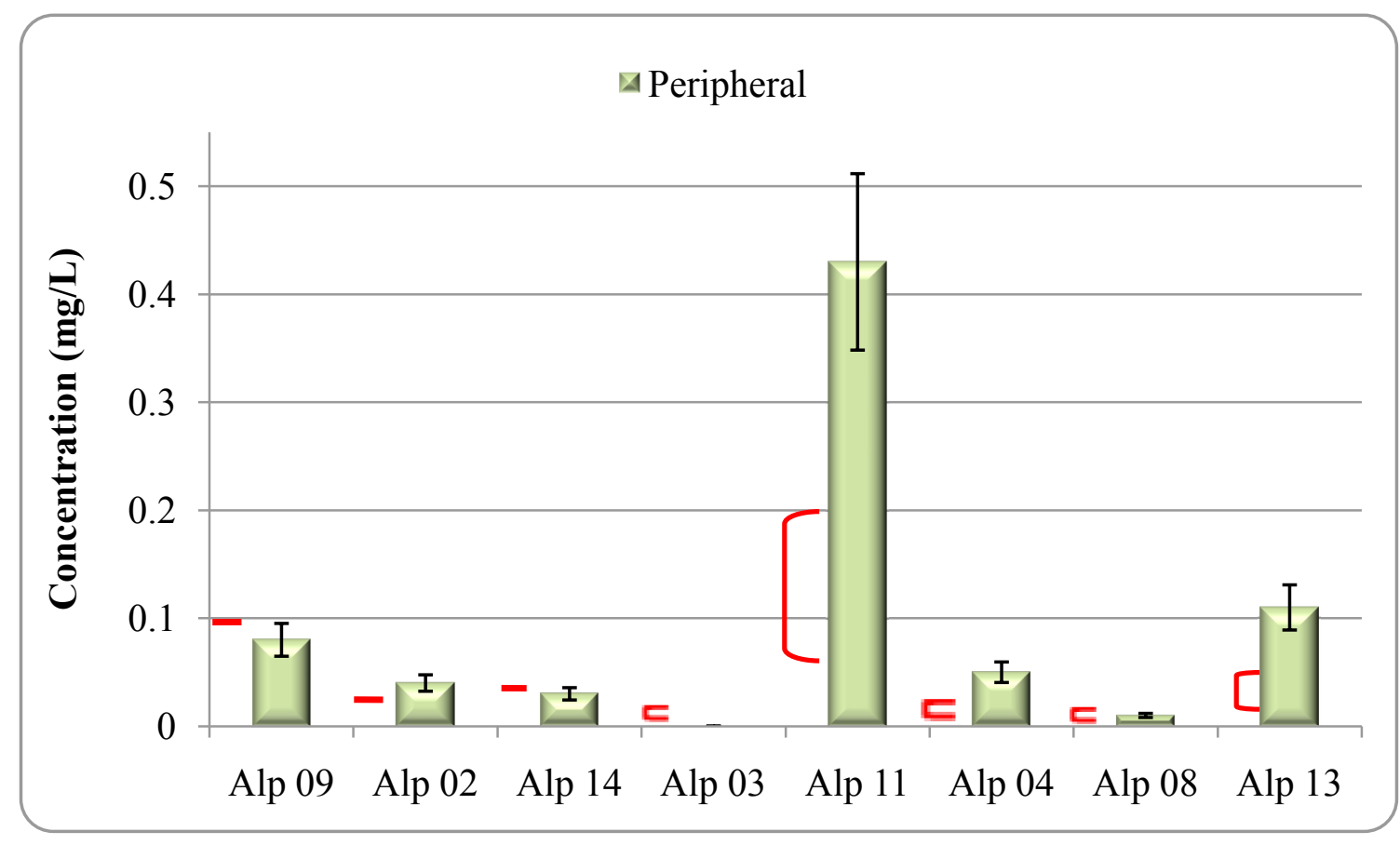

Peripheral alprazolam data as it compares to the expected concentration range at the time of death 
PMR, while the remaining $29 \%$ could not be definitively qualified either way. The collected data seemed to indicate that PMR was not an overwhelming factor for alprazolam. This was consistent with the apparent volume of distribution of the drug ( 0.9 - $1.3 \mathrm{~L} / \mathrm{kg}$ ), which suggested the potential for redistribution, but not necessarily a propensity for it.

\subsubsection{Diazepam}

Diazepam $\left(\right.$ Valium $\left.^{\circledR}\right)$, introduced in the 1960 's, is a long-acting benzodiazepine. Its ability to treat anxiety, muscle spasms and alcohol withdrawal symptoms, help to make it one of the most popular benzodiazepines prescribed. Acceptable therapeutic doses can vary from 2 to $40 \mathrm{mg}$ daily (79). This section will evaluate diazepam and its demethylated metabolite nordiazepam. Elimination half-lives for diazepam and nordiazepam range from $21-37$ hours and $50-99$ hours, respectively (79). The apparent volume of distribution $\left(\mathrm{V}_{\mathrm{d}}\right)$ for diazepam ranges between $0.7-2.6 \mathrm{~L} / \mathrm{kg}(79)$. Typically a $\mathrm{V}_{\mathrm{d}}$ above $1.0 \mathrm{~L} / \mathrm{kg}$ can be indicative of postmortem redistribution and it is possible that diazepam may exhibit some redistributive tendencies. The cases presented here all had antemortem blood collected within one elimination half-life before death. The data is displayed in Table 24 .

Miami-Dade County Medical Examiner Department's Toxicology laboratory conducted nordiazepam and diazepam quantitative testing on a Perkin Elmer HPLC/DAD (section 4.2.2 (b)). While none of the postmortem results were taken from this instrument, due to a finite amount of antemortem sample submitted, some of the cases discussed here did not have sufficient volume for retesting for the purposes of this 
project. As a result, the AM data collected as part of routine laboratory work was included in this section.

Postmortem analysis of nordiazepam and diazepam however, for the purposes of this project, were conducted solely on Thermo-Finnigan's LCQ Advantage HPLC/MS/MS system. In an effort to show that the results obtained from the two methods would not significantly differ from each other a two-way ANOVA (95\% confidence limit) was conducted on the control data collected from both instruments. The nordiazepam ANOVA results (Table 22) which compared the two instruments gave an F-test result of 3.77 and an $\mathrm{F}_{\text {crit }}$ of 4.35. Additionally, Table 23 displays the diazepam ANOVA results in which the F-test and $F_{\text {crit }}$ values for comparing the two instruments were 1.63 and 4.35 , respectively. Both nordiazepam and diazepam had F-test values less than their $F_{\text {crit }}$ values. Therefore, ANOVA determined that no significant difference existed between the two systems. Additionally, the amounts of day to day variability, as well as the interaction between the sources of variation, were not considered significantly different.

Since it was proven that the data obtained on both instruments were comparable, the intra-nordiazepam data as well as the intra-diazepam data were each treated as one population. When using Equation 5 the $\mathrm{CV}$ for nordiazepam and diazepam was $14 \%$ and and $17 \%$, respectively. For each drug, any concentrations that fell within the $\pm \mathrm{CV}$ range were considered similar. Any concentration that exceeded the limits of the range was considered statistically different. 
Table 22: Two-Way Analysis of Variation for Nordiazepam Comparison of HPLC/MS/MS to HPLC/UV Control Data

\begin{tabular}{|cc|cccc|}
\hline Source of Variation & $\begin{array}{c}\text { Sum of } \\
\text { Squares }\end{array}$ & $\boldsymbol{d} f$ & Mean Square & F-test Value & $\boldsymbol{F}$ crit \\
\hline Day to Day & 0.043 & 9 & 0.005 & 0.42 & 2.39 \\
\hline LC/MS vs. LC/UV & 0.044 & 1 & 0.044 & 3.77 & 4.35 \\
\hline Interaction & 0.072 & 9 & 0.008 & 0.69 & 2.39 \\
\hline
\end{tabular}

Table 23: Two-Way Analysis of Variation for Diazepam Comparison of HPLC/MS/MS to HPLC/UV Control Data

\begin{tabular}{|cccccc|}
\hline Source of Variation & $\begin{array}{c}\text { Sum of } \\
\text { Squares }\end{array}$ & $\boldsymbol{d}$ ff & Mean Square & F-test value & F crit \\
\hline Day to Day & 0.13 & 9 & 0.01 & 0.83 & 2.39 \\
\hline LC/MS vs. LC/UV & 0.03 & 1 & 0.03 & 1.63 & 4.35 \\
\hline Interaction & 0.14 & 9 & 0.07 & 0.85 & 2.39 \\
\hline
\end{tabular}


The antemortem blood for diazepam (DZM) case 06 was collected 1.5 hours before death. Nordiazepam and diazepam were detected at concentrations of $0.01 \mathrm{mg} / \mathrm{L}$ each. The expected concentration range at the time of death for nordiazepam was $0.01-$ $0.01 \mathrm{mg} / \mathrm{L}$ (raw data: $0.0098-0.0099 \mathrm{mg} / \mathrm{L}$ ), while diazepam's was $0.01-0.01 \mathrm{mg} / \mathrm{L}$ (raw data: $0.0095-0.0097 \mathrm{mg} / \mathrm{L}$ ). Upon autopsy, heart and iliac vein bloods were submitted. Heart blood had concentrations of $0.03 \mathrm{mg} / \mathrm{L}$ for nordiazepam and $0.02 \mathrm{mg} / \mathrm{L}$ for diazepam. The iliac vein sample had concentrations of $0.02 \mathrm{mg} / \mathrm{L}$ and $0.02 \mathrm{mg} / \mathrm{L}$ for both nordiazepam and diazepam, respectively (Table 24). Both postmortem blood samples had concentrations of nordiazepam and diazepam that were at least twice the amount detected in the antemortem sample. As a result, the heart blood:AM ratios equaled 3.0 and 2.0 for nordiazepam and diazepam, respectively. The iliac vein blood:AM ratios each equaled 2.0 for nordiazepam and diazepam. Additionally, the central to peripheral ratio equaled 1.5 for nordiazepam and 1.0 for diazepam. Even though the PMI was only 6.5 hours, based on the ratio data and the increased PM concentrations, it would appear that PMR did occur.

In addition, the concentration of nordiazepam in DZM 06 was higher in the HTBL than the ILBL. However, statistical analyses determined that the concentrations were not significantly different. The acceptable range of variability for nordiazepam in the HTBL sample was $0.02-0.03 \mathrm{mg} / \mathrm{L}$, while its range in the ILBL sample was $0.02-0.02 \mathrm{mg} / \mathrm{L}$ (raw data $0.017-0.023 \mathrm{mg} / \mathrm{L}$ ). Since the ranges overlapped, the data had to be considered similar.

Antemortem blood for DZM 07 was collected 1.5 hours before death. The blood had a concentration of $0.01 \mathrm{mg} / \mathrm{L}$ for both nordiazepam and diazepam. Heart and iliac 
vein bloods were submitted for PM analysis. The heart blood had $0.01 \mathrm{mg} / \mathrm{L}$ of nordiazepam, as well as $0.01 \mathrm{mg} / \mathrm{L}$ of diazepam. The iliac vein blood had concentrations of $0.01 \mathrm{mg} / \mathrm{L}$ for both nordiazepam and diazepam. As a result, all PM:AM ratios equaled 1.0 , as did the $\mathrm{C}: \mathrm{P}$ ratios.

The blood samples (AM and PM) for DZM 07 were all analyzed on the same day on the LC/MS instrument. Since the results obtained were all quantitatively equal, there were no statistical differences among the values. Even though all of the calculated ratios equaled 1.0, the lack of distinctly different data made it not possible to identify the presence of PMR.

Antemortem blood for DZM 05 was collected 4.5 hours before death. The AM sample had $0.29 \mathrm{mg} / \mathrm{L}$ of nordiazepam and $0.38 \mathrm{mg} / \mathrm{L}$ of diazepam. The expected concentration ranges for nordiazepam and diazepam were $0.27-0.28 \mathrm{mg} / \mathrm{L}$ and $0.33-$ $0.35 \mathrm{mg} / \mathrm{L}$, respectively. Mixed heart and iliac vein bloods were submitted for PM analysis. The mixed heart sample had $0.14 \mathrm{mg} / \mathrm{L}$ of nordiazepam and $0.09 \mathrm{mg} / \mathrm{L}$ of diazepam. The iliac vein blood sample had nordiazepam and diazepam concentrations of $0.07 \mathrm{mg} / \mathrm{L}$ and $0.04 \mathrm{mg} / \mathrm{L}$, respectively. The PM concentrations were below the expected concentration range. Nordiazepam had PM: AM ratios of 0.48 and 0.24 for the MXHT blood and ILBL samples, respectively. Diazepam had PM:AM ratios of 0.24 and 0.10 for MXHT blood and ILBL, respectively. However, the C:P ratios were 2.0 and 2.2 for MXHT and ILBL, respectively.

The AM sample for DZM 05 was analyzed by HLPC/DAD two years prior to the PM analysis by LC/MS. Unfortunately, there was not sufficient quantity of AM sample to submit for re-analysis. As a result, it is likely that during this two year time period the 
PM drug concentrations degraded. Therefore, the comparison of the PM:AM results are probably skewed. However, both PM samples were stored under the same conditions for that two year period. Consequently, the degradation of those two samples should have occurred in a similar fashion. While it cannot be determined for sure that PMR occurred, at the very least the PM results demonstrated that before collection from the body, the mixed heart sample was exposed to more contamination than the iliac vein sample.

Antemortem blood for DZM 04 was collected five hours before death. Diazepam was identified but at levels below the LOQ $(0.01 \mathrm{mg} / \mathrm{L}$ by HPLC/MS $)$. Following autopsy (PMI $=31$ hours), postmortem heart and iliac vein bloods were submitted for analyses. Diazepam was also detected in the heart blood, but at concentrations below the LOQ. The iliac vein sample had $0.01 \mathrm{mg} / \mathrm{L}$ of diazepam. While the concentrations detected in these samples were low, it would appear that some amount of PMR was present in the periphery.

The antemortem blood for DZM 01 had $0.42 \mathrm{mg} / \mathrm{L}$ of nordiazepam and $0.02 \mathrm{mg} / \mathrm{L}$ of diazepam. There were 15 hours between AM collection and death. As a result, the expected concentration ranges at the time of death for nordiazepam would have been $0.34-0.37 \mathrm{mg} / \mathrm{L}$, while diazepam would have been $0.01-0.02 \mathrm{mg} / \mathrm{L}$. Upon autopsy, heart and iliac vein bloods were submitted for analyses. The heart blood sample had 0.19 $\mathrm{mg} / \mathrm{L}$ of nordiazepam and $0.02 \mathrm{mg} / \mathrm{L}$ of diazepam. The iliac vein blood had $0.17 \mathrm{mg} / \mathrm{L}$ of nordiazepam and $0.02 \mathrm{mg} / \mathrm{L}$ of diazepam. The PM nordiazepam concentrations decreased more rapidly than predicted by the calculated results. The resulting PM:AM ratios for nordiazepam were 0.45 and 0.40 for heart blood and iliac vein blood samples, respectively. None of the data produced by nordiazepam was reflective of PMR. 
Table 24: Diazepam and Nordiazepam antemortem samples collected within one elimination half-life Diazepam $\mathrm{t}_{1 / 2}=27-37$ hours $\mathrm{V}_{\mathrm{d}}=0.7-2.6 \mathrm{~L} / \mathrm{kg}$

Nordiazepam $\mathrm{t}_{1 / 2}=50-99$ hours

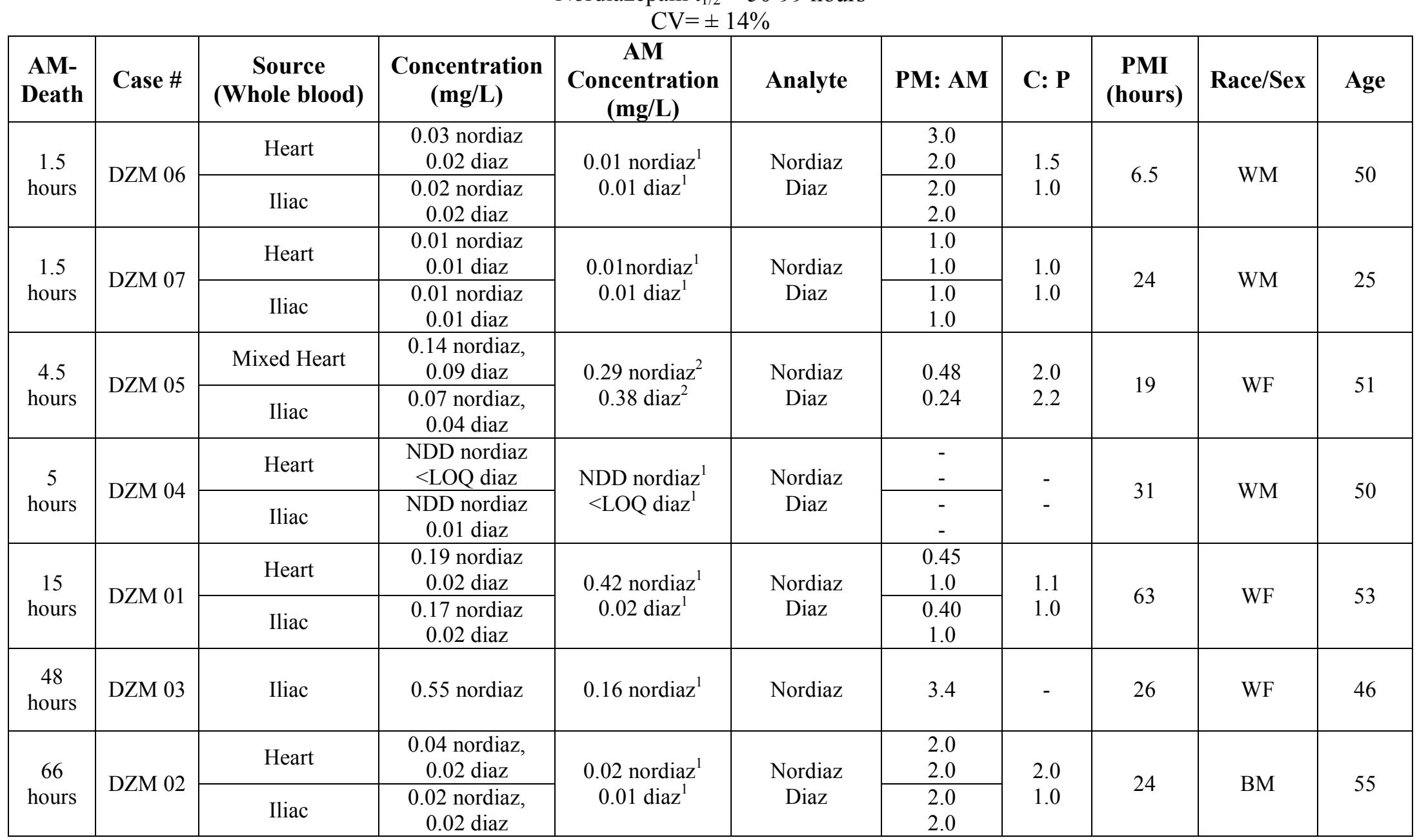

${ }^{1} \mathrm{LC} / \mathrm{MS} / \mathrm{MS}$ analysis (linear range $0.01-1.0 \mathrm{mg} / \mathrm{L}$ ) $\quad{ }^{2} \mathrm{LC} / \mathrm{UV}$ analysis (linear range $0.1-10 \mathrm{mg} / \mathrm{L}$ ) 
The diazepam data for DZM 01 had PM concentrations just within the upper limit of the expected range. The PM:AM ratios for diazepam were 1.0 and 1.0 for heart blood and iliac vein bloods, respectively. While it is believed that ratios with value 1.0 can exhibit PMR, the cumulative diazepam data did not suggest PMR was a factor. What is of particular interest in this case was that both the central and peripheral source blood samples returned concentration data that were similar to each other. This would indicate that the overall change in drug concentration in the body progressed consistently and in the same direction, regardless of the anatomical collection site. This was further reinforced by the C:P ratios of nordiazepam and diazepam equaling 1.1 and 1.0, respectively.

Diazepam case 03 had AM blood collected 48 hours before death. The sample had $0.16 \mathrm{mg} / \mathrm{L}$ of nordiazepam, however diazepam was not detected. While the AMD interval seemed long, it was actually less than the elimination half-life range for nordiazepam (50- 99 hours). Postmortem iliac vein blood was submitted in a gray-top tube and had a nordiazepam concentration of $0.55 \mathrm{mg} / \mathrm{L}$. Diazepam was not detected in the PM sample. The expected concentration of nordiazepam at the time of death would have been between $0.08-0.11 \mathrm{mg} / \mathrm{L}$. The PM concentration well exceeded this range, resulting in a PM:AM ratio of 3.4. The data produced by the ILBL would suggest that PMR occurred during the 26 hour PMI.

Antemortem blood for DZM 02 was collected 66 hours before death. Analysis of the AM blood sample determined that $0.02 \mathrm{mg} / \mathrm{L}$ of nordiazepam and $0.01 \mathrm{mg} / \mathrm{L}$ of diazepam were present. The expected concentration range for nordiazepam was between $0.01-0.01 \mathrm{mg} / \mathrm{L}$, while the expected range for diazepam was below the LOQ. Heart and 
iliac vein bloods were submitted for postmortem analysis. The heart blood sample had $0.04 \mathrm{mg} / \mathrm{L}$ of nordiazepam and $0.02 \mathrm{mg} / \mathrm{L}$ of diazepam. The ILBL sample had $0.02 \mathrm{mg} / \mathrm{L}$ of both nordiazepam and diazepam. The PM:AM ratios for both sources and both drugs equaled 2.0 each. The HTBL concentration for nordiazepam was twice that of the ILBL concentration $(\mathrm{C}: \mathrm{P}=2.0)$. However, diazepam was equal in both sources $(\mathrm{C}: \mathrm{P}=1.0)$. The PM data collected for DZM 02 exceeded the expected concentration ranges for both nordiazepam and diazepam. Additionally, the ratio data in its entirety returned values that were $\geq 1.0$. The data as a whole supports the idea that PMR occurred for both diazepam and nordiazepam.

The discrepancies between the AM and PM data seen in DZM 05 were likely due to the time elapse between AM and PM testing. In any situation, it would be optimal to analyze all the relevant samples for one case within the first few months of collection. However, that did not occur here. Diazepam 05 was still included in this project because it is a reflection of a real world scenario. It is not entirely uncommon for toxicology testing requests to be submitted over a year after a sample has been collected. In these situations, the MDME laboratory complies with the request and will conduct the testing. However, as demonstrated in this case, the analytical results obtained may not be an accurate reflection of the drug concentrations present at the time of sample collection.

Postmortem redistribution was believed to be a factor in diazepam cases 06,03 and 02. All three had PM concentrations at least twice that of their respective AM concentrations, which resulted in PM:AM ratios 2.0. However, their antemortem to death intervals ranged between 1.5 to 66 hours and their postmortem intervals ranged from 6.5-63hours. The large range of the intervals made it difficult to identify a clear 
trend for what factors may contribute to PMR of cases containing diazepam and nordiazepam. It was interesting among these cases that the overall change in concentrations between the two drugs, typically moved in parallel to each other. This is in contradiction to the data produced in the morphine codeine (MOC) cases. The MOC data showed that cases which contained both morphine and codeine could have opposing shifts in concentration, meaning that one drug could decrease in concentration, while the other increased. In diazepam and nordiazepam cases, if one drug increased in concentration, then they both did.

The cumulative diazepam data identified many issues of interest. Firstly, six out of the seven cases analyzed were collected with both central and peripheral sourced postmortem specimens (Chart 10). In $83 \%$ of those cases, the intra-case diazepam data had concentrations that were statistically similar regardless of the source of anatomic collection. Likewise, nordiazepam had $67 \%$ of its intra-case data return statistically similar results regardless of collection site. In the majority of cases researched, the quantitative results would appear to be similar regardless of the PM specimen analyzed. As previously mentioned, the time intervals (AMD and PM) were too broad to definitively identify the reason(s) for concentration discrepancies. While in most instances specimen choice would not matter, for the few instances in which it would, it would be best to use peripheral blood for all quantitative analyses. In addition, also displayed on Chart 10 is DZM 04. Since the AM concentration was below the LOQ, an expected range could not be calculated. However, it would be expected that the PM results would not exceed the AM. The peripheral source had a quantifiable level and that level was included in the chart. 


\section{Chart 10: Diazepam and Nordiazepam Central versus Peripheral Data}

Error rate: $14 \%$ Diazepam, 17\% Nordiazepam

\Central diazepam $₫$ Peripheral diazepam $\square$ Central nordiaz $\square$ Peripheral nordiaz

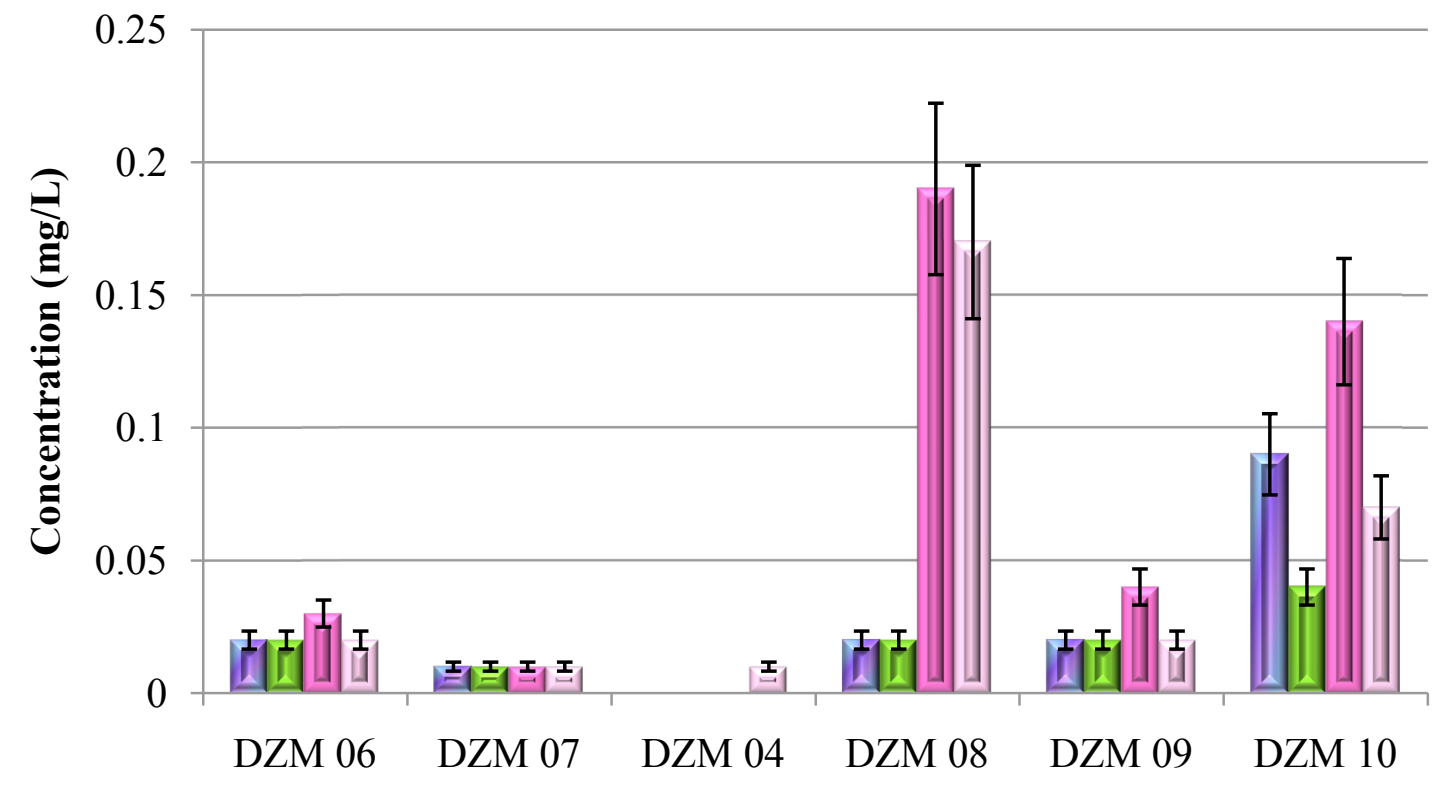




\section{Chart 11: Diazepam and Nordiazepam Peripheral Data}

Error rate: $14 \%$ Diazepam, 17\% Nordiazepam

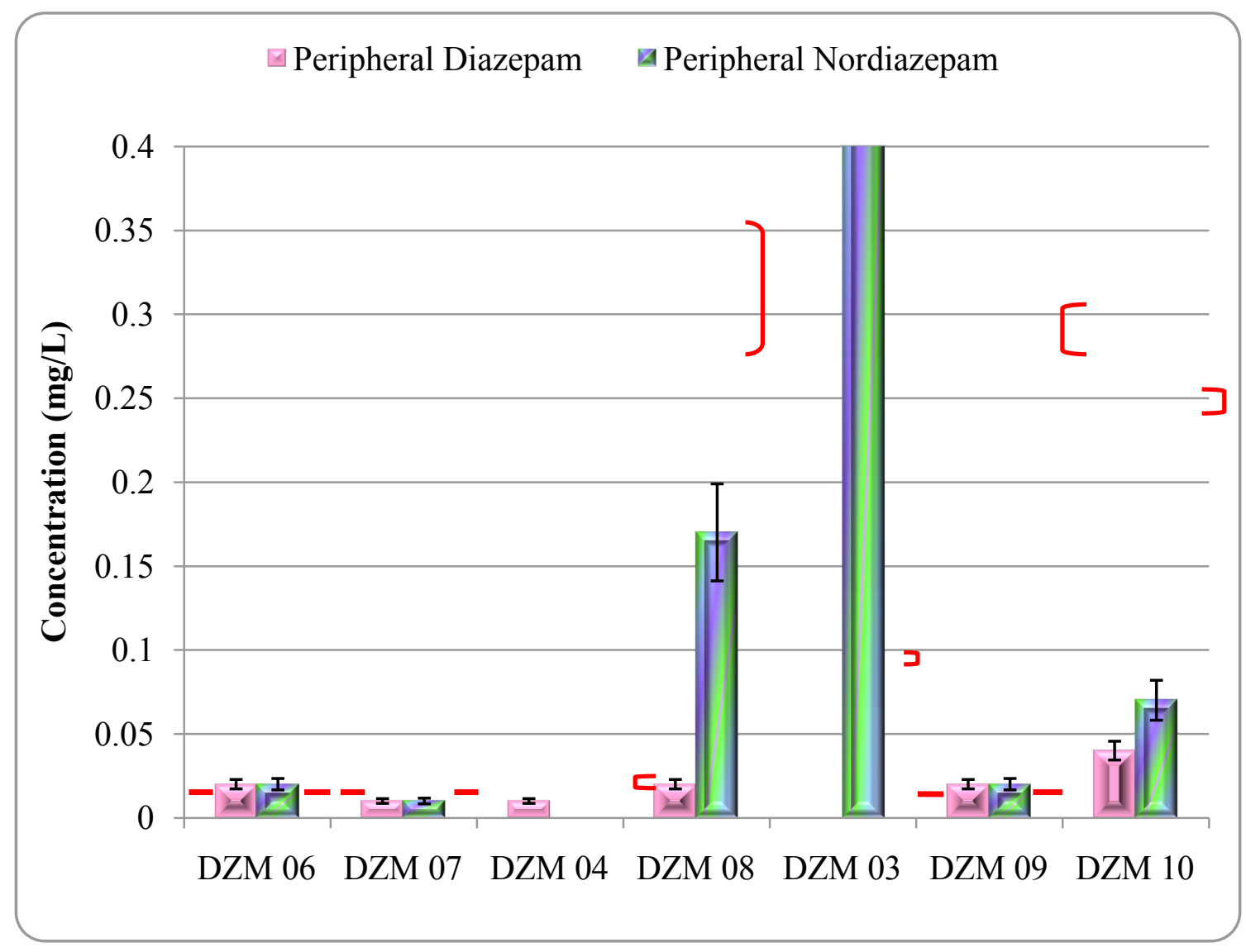

Comparison of diazepam and nordiazepam peripheral data to the expected concentration range at the time of death 
Chart 11 examined the diazepam and nordiazepam peripheral sourced data only. The results of the peripheral data were compared to the calculated expected concentration ranges for each case. The expected ranges are denoted with the symbol "[" for diazepam and "]" for nordiazepam. In some instances the rounded concentrations for the upper and lower levels of the range were equal. For those cases, the symbol "“"” was used for either drug. The data in Chart 11 shows that $50 \%$ of the diazepam PM results were above their expected concentration range, while $17 \%$ was below. The remaining $33 \%$ of the cases produced PM data that was within the expected concentration range at the time of death. Similarly to the diazepam data, the nordiazepam results had 50\% of its PM data above the expected concentration range. In addition, $33 \%$ were below the expected range and $17 \%$ were within range. Based on these results, PM quantitative results for both diazepam and nordiazepam are not likely to be reflective of the drug concentration present in the system at the time of death. To the contrary, it would appear as though peripheral blood data for both drugs are more likely to return results with elevated concentrations.

\subsubsection{Midazolam}

Midazolam is a short-acting benzodiazepine derivative often sold under the trade name Versed ${ }^{\circledR}$. The injection of midazolam into the body is known to rapidly induce sedation. As a result, in the United States, it is often administered by hospitals just prior to surgery. In addition, midazolam (along with other benzodiazepines) triggers smooth muscle relaxation. Consequently, it is often given by paramedics just prior to tracheal intubation of a distressed person. In this situation, the use of midazolam relaxes the gastrointestinal tract muscles, thereby helping to prevent aspiration during intubation 
(80). According to Baselt (81), midazolam has an elimination half-life of $1-4$ hours and an apparent volume of distribution of $1.0-2.5 \mathrm{~L} / \mathrm{kg}$.

For this study, midazolam was quantitated using only Thermo Finnigan's LCQ Advantage HPLC/MS/MS system. While midazolam can be qualitatively identified during the process of routine laboratory work, it is a drug that is not quantitated at the MDME Department's toxicology laboratory. The reason for this is that midazolam is almost exclusively given in a hospital setting and generally not prescribed in the United States. For that reason, its potential for abuse is extremely low. However, since one of the criteria for this study was the need of antemortem blood (which could only be drawn in a hospital setting) midazolam was one of the most commonly encountered drugs in the project. As a result, the researcher decided to collect quantitative data (linear range 0.01 $-1.0 \mathrm{mg} / \mathrm{L})$. Only one control lot, and one instrument, was used during the analysis process. Therefore, ANOVA processing was not needed. However, the CV of the population was determined to be $18 \%$ (Table 25). All drug concentrations that fell within a $\pm 18 \%$ range were determined to be statistically similar. The cases presented can be found in Table 26, listed in order of increasing antemortem to death interval.

Table 25: Midazolam Control Data (0.1 mg/L) Statistics

\begin{tabular}{|cc|}
\hline Concentration Mean & $0.145571 \mathrm{mg} / \mathrm{L}$ \\
\hline Standard Deviation & 0.026144 \\
\hline CV & $\pm 18 \%$ \\
\hline
\end{tabular}

Midazolam (MDZ) 01 had antemortem blood collected 16 minutes before death. The AM sample had a midazolam concentration of $0.29 \mathrm{mg} / \mathrm{L}$. The expected 
concentration at the time of death would have ranged between $0.24-0.28 \mathrm{mg} / \mathrm{L}$. Upon autopsy, heart and iliac vein blood samples were collected and submitted for analyses (PMI $=26$ hours). The heart blood sample had a concentration of $0.05 \mathrm{mg} / \mathrm{L}$, while the iliac vein blood had $0.06 \mathrm{mg} / \mathrm{L}$ of midazolam. The PM to AM ratio equaled 0.16 for the HTBL and 0.20 for the ILBL. Additionally, the central to peripheral ratio equaled 0.79. When examining the quantitative data, it was apparent that the ILBL concentration was higher than the HTBL concentration. This would lead one to believe that in the case of PMR, the peripheral blood was more greatly affected. However, statistical analysis determined that the HTBL and ILBL concentrations were, in fact, similar. The concentration effects were similar regardless of anatomical collection site.

The overall data produced in MDZ 01 reflected a lack of PMR. This was further demonstrated by the ratio data, in which all ratios had values $<1.0$. Additionally, the PM concentrations were not only less than the AM concentration, but they were significantly below the expected concentration range at the time of death.

The antemortem blood for MDZ 04 was collected two hours before death and had $0.16 \mathrm{mg} / \mathrm{L}$ of midazolam present. The expected drug concentration at the time of death ranged between 0.04 to $0.11 \mathrm{mg} / \mathrm{L}$. Heart and iliac vein blood samples were collected at autopsy. Postmortem blood concentrations equaled $0.02 \mathrm{mg} / \mathrm{L}$ and $0.01 \mathrm{mg} / \mathrm{L}$ for heart and iliac vein samples, respectively. The central to peripheral ratio equaled 1.6 , while the HTBL:AM and ILBL:AM ratios equaled 0.14 and 0.09 , respectively. The C:P ratio results were indicative of PMR, however both PM: AM ratios combated this. The researcher determined that since the PM concentrations obtained were significantly less than expected, PMR did not occur during the 23 hour PMI. 
Table 26: Midazolam Cases listed in order of increasing AM to death intervals

$$
\begin{gathered}
\mathrm{t}_{1 / 2}=1-4 \text { hours } \\
\mathrm{V}_{\mathrm{d}}=1.0-2.5 \mathrm{~L} / \mathrm{kg}
\end{gathered}
$$

Linear range: $0.01-1.0 \mathrm{mg} / \mathrm{L}$

\begin{tabular}{|c|c|c|c|c|c|c|c|c|c|c|}
\hline $\begin{array}{l}\text { AM- } \\
\text { Death }\end{array}$ & Case \# & $\begin{array}{c}\text { Source } \\
\text { (Whole Blood) }\end{array}$ & $\begin{array}{c}\text { Concentration } \\
(\mathrm{mg} / \mathrm{L})\end{array}$ & $\begin{array}{c}\text { AM } \\
\begin{array}{c}\text { Concentration } \\
(\mathrm{mg} / \mathrm{L})\end{array}\end{array}$ & Analyte & PM: AM & C: $P$ & $\begin{array}{c}\text { PMI } \\
\text { (hours) }\end{array}$ & Race/Sex & Age \\
\hline \multirow{2}{*}{$16 \mathrm{~min}$} & \multirow{2}{*}{$\begin{array}{c}\text { MDZ } \\
01\end{array}$} & Heart & 0.05 & \multirow{2}{*}{0.29} & \multirow{2}{*}{ AMBL } & 0.16 & \multirow{2}{*}{0.79} & \multirow{2}{*}{26} & \multirow{2}{*}{$\mathrm{BF}$} & \multirow{2}{*}{44} \\
\hline & & Iliac & 0.06 & & & 0.20 & & & & \\
\hline \multirow{2}{*}{$2 \mathrm{hrs}$} & \multirow{2}{*}{$\begin{array}{c}\text { MDZ } \\
04\end{array}$} & Heart & 0.02 & \multirow{2}{*}{0.16} & \multirow{2}{*}{ AMBL } & 0.14 & \multirow{2}{*}{1.6} & \multirow{2}{*}{23} & \multirow{2}{*}{$\mathrm{BM}$} & \multirow{2}{*}{37} \\
\hline & & Iliac & 0.01 & & & 0.09 & & & & \\
\hline \multirow{2}{*}{$3.5 \mathrm{hrs}$} & MDZ & Mixed & $<\mathrm{LOQ}$ & \multirow{2}{*}{0.02} & \multirow{2}{*}{ AMBL } & - & \multirow{2}{*}{ - } & \multirow{2}{*}{17} & \multirow{2}{*}{ WF } & \multirow{2}{*}{50} \\
\hline & 08 & Iliac & $<\mathrm{LOQ}$ & & & - & & & & \\
\hline \multirow{2}{*}{$6 \mathrm{hrs}$} & MDZ & Mixed & $<\mathrm{LOQ}$ & \multirow{2}{*}{0.01} & \multirow{2}{*}{ AMBL } & - & \multirow{2}{*}{ - } & \multirow{2}{*}{10} & \multirow{2}{*}{$\mathrm{BM}$} & \multirow{2}{*}{25} \\
\hline & 03 & Aorta & $<\mathrm{LOQ}$ & & & - & & & & \\
\hline \multirow{2}{*}{$6.5 \mathrm{hrs}$} & MDZ & Aorta & $<\mathrm{LOQ}$ & \multirow{2}{*}{0.01} & \multirow{2}{*}{ AMBL } & - & \multirow[b]{2}{*}{ - } & \multirow{2}{*}{33.5} & \multirow{2}{*}{$\mathrm{BM}$} & \\
\hline & 07 & Iliac & $<\mathrm{LOQ}$ & & & - & & & & 38 \\
\hline $30 \mathrm{hrc}$ & MDZ & Mixed & $<\mathrm{LOQ}$ & 006 & $\triangle M R I$ & 1.0 & 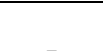 & 37 & WM & 30 \\
\hline $30 \mathrm{hrs}$ & 06 & Aorta & $<$ LOQ & 0.06 & AIMBL & 1.0 & - & 31 & $\mathrm{WM}$ & 39 \\
\hline 2 days & $\begin{array}{c}\mathrm{MDZ} \\
02\end{array}$ & Heart* & $<$ LOQ & 0.06 & AMBL & - & - & 32 & WM & 83 \\
\hline unknown & $\begin{array}{c}\text { MDZ } \\
05\end{array}$ & Aorta & 0.32 & 0.05 & AMBL & 6.4 & - & 43 & WM & 29 \\
\hline
\end{tabular}

$$
\mathrm{CV}= \pm 18 \%
$$

* Average concentration 
Antemortem blood from MDZ 08 was collected 3.5 hours before death and had a concentration of $0.02 \mathrm{mg} / \mathrm{L}$. The expected concentration range at the time of death was between $1.8 \times 10^{-3}-0.01 \mathrm{mg} / \mathrm{L}$. A mixed blood sample, as well as an iliac vein blood sample was submitted for PM analyses. Both PM samples had blood concentrations less than the LOQ. This was consistent with the calculated expected concentration range, as only the upper range value was within the LOQ and it was determined that PMR was not present in this case.

Antemortem blood for MDZ 03 had a concentration of $0.01 \mathrm{mg} / \mathrm{L}$. Since the AMBL was collected 6 hours before death, it had an expected concentration range of 3.8 x $10^{-8}-4.4 \times 10^{-4} \mathrm{mg} / \mathrm{L}$, well below the LOD $(0.002 \mathrm{mg} / \mathrm{L})$. Mixed and aorta bloods were submitted post autopsy. Midazolam was detected in both samples, but at levels below the LOQ. The fact that midazolam was detected, indicated that the concentration was higher than the calculated expected range. However, the PM concentrations were still below the AM concentration. As a result, it was determined that PMR was not a factor.

A concentration of $0.01 \mathrm{mg} / \mathrm{L}$ was detected in the AM blood collected 6.5 hours before death in midazolam case 07 . The low AM concentration in combination with the amount of time that passed during the antemortem to death interval caused the expected concentration range at the time of death $\left(1.1 \times 10^{-4}-3.2 \times 10^{-3} \mathrm{mg} / \mathrm{L}\right)$ to be below the LOD. Aorta and iliac vein bloods were analyzed and returned concentrations below the LOQ. Therefore PMR did not occur.

In midazolam 06, the AM blood was collected 30 hours before death and had an AM concentration of $0.06 \mathrm{mg} / \mathrm{L}$. If no additional midazolam was administered during the 
AMD interval, the expected concentration range at the time of death would have been between $5.6 \times 10^{-11}-3.3 \times 10^{-4} \mathrm{mg} / \mathrm{L}$. There was a 37 hour PMI in this case. After which, mixed and aorta bloods were collected and submitted for testing. Both PM specimens had blood drug concentrations that were less than the limit of quantitation. This was consistent with the expected concentration range.

Antemortem blood for MDZ 02 had a concentration of $0.06 \mathrm{mg} / \mathrm{L}$ and was collected 2 days before death. The expected midazolam concentration at the time of death was between $5.6 \times 10^{-11}-3.5 \times 10^{-4} \mathrm{mg} / \mathrm{L}$. Analysis of the heart blood sample submitted post autopsy was positive for midazolam, however at a level below that of quantitation. The expected concentration range was below the LOD. While it would appear that the PM concentration was higher than expected, it was still at least 6 times below that of the AM sample. As a result, it was determined that PMR did not occur.

The AM blood for midazolam 05 was submitted with only a collection time on it, not a date. Upon checking the hospital records, the decedent was a patient there for approximately 48 hours. Based on the time written on the tube, the AM sample was either collected 32 hours or 8 hours before death. Since the exact date of collection cannot be resolved, an expected concentration range was not calculated. The AM sample however, did have a midazolam concentration of $0.05 \mathrm{mg} / \mathrm{L}$. As a result, it would be expected that the PM concentrations would be below this value. Aorta blood was submitted in both the red-top and gray-top blood collection tubes post autopsy. The midazolam concentration was $0.31 \mathrm{mg} / \mathrm{L}$ and $0.34 \mathrm{mg} / \mathrm{L}$ for red-top and gray-top tubes, respectively. Since the tubes were of the same anatomical source and the concentrations were statistically similar, the overall midazolam concentration was averaged to 
$0.32 \mathrm{mg} / \mathrm{L}$. The increased PM concentration (relative to the AM concentration) resulted in a PM:AM ratio of 6.4. A review of the medical records only indicated one administration of midazolam. Therefore, the increase in the postmortem sample cannot be explained by re-administration. In the absence of any contradictory information, it would appear as though PMR occurred during the 43 hour PMI. If so, this would be the only midazolam case researched that exhibited this phenomena.

Although the apparent volume of distribution for midazolam $(1.0-2.5 \mathrm{~L} / \mathrm{kg})$ does suggest that the drug could have some redistributive effects, with the exception of MDZ 05, the data collected indicated otherwise. Eighty-eight percent of the cases examined showed no signs of PMR. Although there were a few instances where the PM concentrations were higher than their expected calculated ranges (MDZ 08, 03, 07 and 02), those concentrations were still below their respective antemortem concentrations. Only MDZ 05 had a PM concentration that exceeded its AM level. The uncertainty of the actual AMD interval for that case makes it difficult to compare to the others. Its PMI, however, was noted as being 43 hours, which would make it the longest PMI examined. If the true AMD interval equaled eight hours (as opposed to 32 hours) then this case would have a relatively short AMD coupled with a long PMI. This was a combination believed to influence PMR of alprazolam. Further studies of midazolam cases would be needed before any formal conclusions could be drawn.

Additionally, midazolam cases $01(\mathrm{PMI}=26$ hours $)$ and $13(\mathrm{PMI}=23$ hours $)$ were the only two case that returned quantifiable data for both central and peripheral sourced samples. In MDZ 01, the concentrations of midazolam obtained in both sources were statistically similar to each other, indicating that concentration changes between the 
two regions of the body were not significantly different. In that case, however, the AMD interval was only 16 minutes. In MDZ 04, the AMD interval was slightly more pronounced at two hours. The PM results obtained in that case did indicate statistically different results depending on the anatomical region of collection. As would be expected based on conventional theory, the central sourced blood had a higher amount of midazolam (two times greater) than the peripheral blood. While neither case rendered results indicative of PMR, the two cases did show that under certain circumstances, PM concentrations may still have a tendency to differ in concentration based on anatomical location.

Sixty-three percent of the PM data collected was qualitative. Since this research was primarily based on quantitative data, the usefulness of qualitative data may not be readily apparent. In the instance of midazolam, PM data that was below the limit of quantitation, although within the limit of detection was reflective of an overall decrease in midazolam blood concentrations after antemortem sample collection. Based on the results of this study, generally midazolam did not appear to store itself within the tissues

of the body. This was primarily of interest because the range of the apparent volume of distribution suggested otherwise.

\subsubsection{Clonazepam and 7-aminoclonazepam}

Clonazepam is a long acting nitrobenzodiazepine $\left(\mathrm{t}_{1 / 2}=19-60\right.$ hours $)(61)$ often used to treat epilepsy, anxiety and sleep disorders. 7-aminoclonazepam is the pharmacologically inactive major metabolite of clonazepam, which is produced by a reduction reaction (62). Nitrobenzodiazepines (including clonazepam) have been known 
to reduce to their 7-amino analogs in storage, even when preserved (21)(33)(63). This fact makes the interpretation of these drugs more difficult than other benzodiazepines discussed in this manuscript. The elimination half-life of 7 -aminoclonazepam could not be found in the literature prior to the writing of this section. It is possible that since the drug is not believed to be active, its rate of elimination has not been extensively studied. Equation 7 was used to calculate the expected concentration range of clonazepam only, not 7-aminoclonazepam. Quantitative analyses of both drugs however, were conducted on both the GC/ECD and HPLC/MS. A two-way ANOVA (95\% confidence) was conducted on the control data collected from both instruments to determine if the data was similar enough to be treated as one population.

The results obtained from the two-way ANOVA indicated that the data collected on the two instruments were dis-similar. This is illustrated in Table 27 (clonazepam) and Table 28 (7-aminoclonazepam). The clonazepam data had an F-test value of 26.8 and an $\mathrm{F}_{\text {crit }}$ of 4.3 when comparing the two instruments. Likewise, 7-aminoclonazepam had an F-test value of 8.8 and an $F_{\text {crit }}$ value of 4.3. Anytime an F-test value is greater than its $\mathrm{F}_{\text {crit, }}$, the null hypothesis (that is, there is no difference between the two) is rejected, as was the case here. In an effort to verify the F-test results, Tukey's post hoc analysis was conducted as described in section 5.0 Calculations. Tukey's critical value for clonazepam was 0.012 , with a mean difference of 0.030 . 7-aminoclonazepam's critical value was 0.004 and had a mean difference of 0.007 . For both clonazepam and 7aminoclonazepam, Tukey's critical value was less than the mean difference, indicating that a difference between the instruments did in fact exist. Therefore, it was determined that the ANOVA calculations were accurate. As a result, the data produced on each 
Table 27: Two-Way Analysis of Variation for Clonazepam Comparison of GC/ECD to $\mathrm{LC} / \mathrm{MS} / \mathrm{MS}$ control Data

\begin{tabular}{|cccccccc|}
\hline $\begin{array}{c}\text { Source of } \\
\text { Variation }\end{array}$ & $\begin{array}{c}\text { Sum of } \\
\text { Squares }\end{array}$ & df & $\begin{array}{c}\text { Mean } \\
\text { Square }\end{array}$ & $\begin{array}{c}\text { F-test } \\
\text { value }\end{array}$ & F crit & $\begin{array}{c}\text { Tukey's } \\
\text { critical } \\
\text { value }\end{array}$ & $\begin{array}{c}\text { Mean } \\
\text { Difference }\end{array}$ \\
\hline Day to Day & 0.001 & 9 & 0.0002 & 0.42 & 2.39 & & \\
\hline $\begin{array}{c}\text { GC/ECD } \\
\text { vs. LC/MS }\end{array}$ & 0.011 & 1 & 0.0107 & 26.86 & 4.35 & 0.012 & 0.030 \\
\hline Interaction & 0.002 & 9 & 0.0002 & 0.50 & 2.39 & & \\
\hline
\end{tabular}

Table 28: Two-Way Analysis of Variation for 7-aminoclonazepam Comparison GC/ECD to LC/MS/MS control Data

\begin{tabular}{|cccccccc|}
\hline $\begin{array}{c}\text { Source of } \\
\text { Variation }\end{array}$ & $\begin{array}{c}\text { Sum of } \\
\text { Squares }\end{array}$ & $d f$ & $\begin{array}{c}\text { Mean } \\
\text { Square }\end{array}$ & $\begin{array}{c}\text { F-test } \\
\text { value }\end{array}$ & F crit & $\begin{array}{c}\text { Tukey's } \\
\text { critical } \\
\text { value }\end{array}$ & $\begin{array}{c}\text { Mean } \\
\text { Difference }\end{array}$ \\
\hline Day to Day & 0.0002 & 9 & $2.34 \mathrm{E}-05$ & 0.57 & 2.39 & & \\
\hline $\begin{array}{c}\text { GC/ECD vs. } \\
\text { LC/MS }\end{array}$ & 0.0004 & 1 & 0.0004 & 8.83 & 4.35 & 0.004 & 0.007 \\
\hline Interaction & 0.0009 & 9 & 0.0001 & 2.58 & 2.39 & & \\
\hline
\end{tabular}


instrument was treated separately. Clonazepam cases analyzed by GC/ECD had a CV of $15 \%$, while 7-aminoclonazepam had a $\mathrm{CV}$ of $17 \%$. The clonazepam and 7aminoclonazepm data collected by LC/MS had CV's of $15 \%$ and $21 \%$, respectively (refer to Table 29).

Table 29: Coefficient of Variation for Clonazepam and 7-aminoclonazepam

\begin{tabular}{c|cc|}
\multicolumn{1}{c}{ Analyte } & Instrument & CV \\
\hline Clonazepam & GC/ECD & $15 \%$ \\
& LC/MS & $15 \%$ \\
\hline 7-aminoclonazepam & GC/ECD & $17 \%$ \\
& LC/MS & $21 \%$ \\
\cline { 2 - 3 }
\end{tabular}

The data from the clonazepam (CNP) cases used in this study can be seen in Table 30. The cases are presented in order of increasing antemortem to death (AMD) interval. Whenever sample quantity allowed, AM samples were re-analyzed at or around the same time as the PM analyses. On occasion the sample volume was not sufficient for this and the concentration obtained as a part of routine analysis was used. The results obtained from re-analyzed AM samples are discussed on a case-by-case basis.

Clonazepam (CNP) case 04 had AM blood collected 10 minutes before death. The sample had $0.03 \mathrm{mg} / \mathrm{L}$ of clonazepam and $0.13 \mathrm{mg} / \mathrm{L}$ of 7 -aminoclonazepam. The expected concentration range for clonazepam at the time of death would have been between $0.03-0.03 \mathrm{mg} / \mathrm{L}$ (raw data: $0.0298-0.0299 \mathrm{mg} / \mathrm{L}$ ). Postmortem iliac vein blood was submitted in both the gray and red-top collection tubes (PMI $=28.5$ hours). The red-top ILBL had concentrations of $0.02 \mathrm{mg} / \mathrm{L}$ and $0.15 \mathrm{mg} / \mathrm{L}$ for clonazepam and 7aminoclonazepam, respectively. The gray-top ILBL had $0.02 \mathrm{mg} / \mathrm{L}$ of clonazepam and 
$0.23 \mathrm{mg} / \mathrm{L}$ of 7-aminoclonazepam. Although the PM sources were the same, the 7aminoclonazepam concentrations were statistically different from each other $( \pm 17 \%$ by GC/ECD). Therefore the concentrations from both tubes could not be averaged. The 7aminoclonazepam concentration discrepancy in the PM specimens was of interest because as reported by Drummer (21) and Steentoft (64), 7-aminoclonazepam can be formed PM even in preserved blood. It is possible that the GT ILBL had more 7aminoclonazepam production than the RT ILBL.

When comparing the PM data to that of the AM, all of the concentrations, with the exception of the RT ILBL 7-aminoclonazepam concentration, were determined to be statistically dis-similar to the AM. The PM:AM ratios for the RT ILBL were 0.67 and 1.1 for clonazepam and 7-aminoclonazepam, respectively. The GT ILBL had PM:AM ratios of 0.67 and 1.8 for clonazepam and 7-aminoclonazepam, respectively. By convention, it is believed that ratio results that are greater than or equal to 1.0 are reflective of PMR. Therefore, despite the discrepancy between the actual values obtained in the PM samples, both tubes seem to be in agreement that some amount of redistribution affected 7-aminoclonazepam, while none influenced clonazepam. The fact that for both sources clonazepam decreased below its expected range and 7aminoclonazepam increased caused the researcher to determine that despite the ratio data, PMR did not influence either drug in this case. Instead, the results were indicative of chemical conversion between the two drugs.

The AM blood for CNP 07 was collected one hour before death and was analyzed as a part of routine analysis by GC/ECD. The sample was determined to have concentrations of $0.03 \mathrm{mg} / \mathrm{L}$ and $0.02 \mathrm{mg} / \mathrm{L}$ for clonazepam and 7-aminoclonazepam, 
Table 30: Clonazepam antemortem samples collected within two elimination half-lives

Clonazepam: $\mathrm{V}_{\mathrm{d}}=1.5-4.4 \mathrm{~L} / \mathrm{kg} ; \mathrm{t}_{1 / 2}=19-60$ hours

7-aminoclonazepam: $\mathrm{V}_{\mathrm{d}}$ and $\mathrm{t}_{1 / 2}=$ unknown

\begin{tabular}{|c|c|c|c|c|c|c|c|c|c|c|}
\hline $\begin{array}{c}\text { AM - } \\
\text { Death } \\
\text { (hours) }\end{array}$ & $\begin{array}{c}\text { Case } \\
\#\end{array}$ & $\begin{array}{l}\text { Source } \\
\text { (whole } \\
\text { blood) }\end{array}$ & $\begin{array}{c}\text { Concentration } \\
(\mathrm{mg} / \mathrm{L})\end{array}$ & $\begin{array}{c}\mathrm{AM} \\
\text { concentration } \\
(\mathrm{mg} / \mathrm{L}) \\
\end{array}$ & Specimen & PM: AM & $C: P$ & $\begin{array}{c}\text { PMI } \\
\text { (hours) }\end{array}$ & Race/Sex & Age \\
\hline \multirow{2}{*}{$10 \min$} & \multirow{2}{*}{$\begin{array}{l}\text { CNP } \\
04^{1}\end{array}$} & Iliac $_{\mathrm{RT}}$ & $\begin{array}{c}0.02 \text { clonaz, } \\
0.15 \text { (7amino) }\end{array}$ & \multirow{2}{*}{$\begin{array}{c}0.03 \text { clonaz } \\
0.13 \text { (7amino) }\end{array}$} & \multirow{2}{*}{ AMBL } & $\begin{array}{c}0.67 \text { clonaz } \\
1.1 \text { (7amino) }\end{array}$ & - & \multirow{2}{*}{28.5} & \multirow{2}{*}{ WM } & \multirow{2}{*}{44} \\
\hline & & Iliac $_{\mathrm{GT}}$ & $\begin{array}{c}0.02 \text { clonaz, } \\
0.23 \text { (7amino) }\end{array}$ & & & $\begin{array}{c}0.67 \text { clonaz } \\
1.8 \text { (7amino })\end{array}$ & - & & & \\
\hline \multirow{2}{*}{1 hour } & \multirow{2}{*}{$\begin{array}{c}\text { CNP } \\
07^{2}\end{array}$} & Heart & 0.05 (7amino) & \multirow{2}{*}{$\begin{array}{c}0.03 \text { clonaz } \\
0.02 \text { (7amino) }\end{array}$} & \multirow{2}{*}{ AMBL } & 2.5 (7amino) & 0.83 & \multirow{2}{*}{24} & \multirow{2}{*}{ WM } & \multirow{2}{*}{25} \\
\hline & & Iliac & 0.06 (7amino) & & & 3.0 (7amino) & (7amino) & & & \\
\hline \multirow{2}{*}{$\begin{array}{c}1.5 \\
\text { hours }\end{array}$} & \multirow{2}{*}{$\begin{array}{l}\text { CNP } \\
06^{2} \\
\end{array}$} & Heart & 0.04 (7amino) & \multirow{2}{*}{0.04 (7amino) } & \multirow{2}{*}{ AMBL } & 1.0 (7amino) & 1.0 & \multirow{2}{*}{6.5} & \multirow{2}{*}{ WM } & \multirow{2}{*}{50} \\
\hline & & Iliac & 0.04 (7amino) & & & 1.0 (7amino) & (7amino) & & & \\
\hline 6 hours & $\begin{array}{c}\text { CNP } \\
02^{1}\end{array}$ & Heart* & 0.03 clonaz & 0.01 clonaz & AMBL & 3.0 clonaz & - & 70.5 & WF & 22 \\
\hline \multirow{2}{*}{$\begin{array}{c}12 \\
\text { hours }\end{array}$} & \multirow{2}{*}{$\begin{array}{l}\text { CNP } \\
05^{1}\end{array}$} & Aorta & $\begin{array}{c}0.08 \text { clonaz, } \\
0.04 \text { (7amino) }\end{array}$ & \multirow{2}{*}{$\begin{array}{l}<\mathrm{LOQ} \text { clonaz } \\
0.17 \text { (7amino) }\end{array}$} & \multirow{2}{*}{ AMBL } & $\begin{array}{c}0.23 \\
\text { (7amino) } \\
\end{array}$ & $\begin{array}{c}4.0 \\
\text { clonaz }\end{array}$ & \multirow{2}{*}{54} & \multirow{2}{*}{ WM } & \multirow{2}{*}{68} \\
\hline & & Iliac & $\begin{array}{c}0.02 \text { clonaz, } \\
0.47 \text { (7amino) }\end{array}$ & & & 2.8 (7amino) & $\begin{array}{c}0.08 \\
\text { (7amino) }\end{array}$ & & & \\
\hline \multirow{2}{*}{$\begin{array}{c}14 \\
\text { hours }\end{array}$} & \multirow{2}{*}{$\begin{array}{c}\text { CNP } \\
03^{1} \\
\end{array}$} & Mixed & NDD & \multirow{2}{*}{0.01 clonaz } & \multirow{2}{*}{ AMBL } & - & \multirow[t]{2}{*}{-} & \multirow{2}{*}{19} & \multirow{2}{*}{ WF } & \multirow{2}{*}{49} \\
\hline & & Iliac & NDD & & & - & & & & \\
\hline 15 & CNP & Heart & $\begin{array}{c}0.02 \text { clonaz, } \\
0.17 \text { (7amino) }\end{array}$ & 0.15 clonaz & PI & $\begin{array}{c}0.13 \text { clonaz } \\
3.4 \text { (7amino) }\end{array}$ & $\begin{array}{c}1.0 \\
\text { clonaz }\end{array}$ & 62 & WE & 52 \\
\hline hours & $08^{2}$ & Iliac & $\begin{array}{c}0.02 \text { clonaz, } \\
0.10 \text { (7amino) }\end{array}$ & 0.05 (7amino) & AIVIBL & $\begin{array}{c}0.13 \text { clonaz } \\
2.0 \text { (7amino) }\end{array}$ & $\begin{array}{c}1.7 \\
\text { (7amino) }\end{array}$ & 03 & WF & 53 \\
\hline 66 & CNP & Heart & 0.04 (7amino) & 094 (7amino) & & 1.0 (7amino) & 1.0 & & $\mathrm{DN}$ & \\
\hline hours & $09^{2}$ & Iliac & 0.04 (7amino) & no) & IBL & 1.0 (7amino) & (7amino) & 24 & BMI & 5 \\
\hline
\end{tabular}

${ }^{1} \mathrm{GC} / \mathrm{ECD}$ (linear range: $0.01-0.2 \mathrm{mg} / \mathrm{L}$ )

${ }^{2} \mathrm{LC} / \mathrm{MS}$ (linear range: $0.01-1.0 \mathrm{mg} / \mathrm{L}$ )

* Average concentration 
respectively. The expected concentration of clonazepam at the time of death would have been between $0.03-0.03 \mathrm{mg} / \mathrm{L}$ (raw data: $0.0289-0.0296 \mathrm{mg} / \mathrm{L}$ ). However, there was sufficient quantity of the AM blood sample to be re-tested for research purposes.

Four months after the original analysis of CNP 07, AM blood as well as PM heart and iliac vein bloods (PMI = 24 hours) were analyzed (as part of one batch) by LC/MS. At that time the AM blood concentration for 7-aminoclonazepam was $0.05 \mathrm{mg} / \mathrm{L}$, while clonazepam was not detected (LOD by LC/MS $=0.002 \mathrm{mg} / \mathrm{L}$ ). The heart blood sample had $0.05 \mathrm{mg} / \mathrm{L}$ of 7-aminoclonazepam and the iliac vein blood had $0.06 \mathrm{mg} / \mathrm{L}$ of 7 aminoclonazepam. The central to peripheral ratio equaled 0.83 . Clonazepam was not detected in either PM sample.

In, and of itself, the absence of clonazepam in the re-test of the AM sample would be indicative of drug instability of the drug in the AM tube during the 4 month storage period. However, the decrease of clonazepam was coupled with a 50\% increase in 7aminoclonazepam. It is more likely that drug reduction in the AM sample occurred. Also of interest was that the PM samples rendered concentrations that were statistically similar to each other, as well as being similar to the re-tested AM values. The data produced for this case was not indicative of PMR, rather the apparent conversion of clonazepam into 7-aminoclonazepam caused this concentration discrepancy.

The AM and PM samples for CNP 06 were analyzed on the same day as part of one batch on the HPLC/MS system. The AM sample had $0.04 \mathrm{mg} / \mathrm{L}$ of 7aminoclonazepam while clonazepam was not detected. Since the elimination half-life for 7-aminoclonazepam is not known, an AMD interval could not be calculated. However, the AMD interval was only 1.5 hours, suggesting that the expected concentration range at 
the time of death would not have deviated significantly from the AM. Heart and iliac vein bloods were submitted post autopsy (PMI $=6$ hours). Both PM samples had concentrations of $0.04 \mathrm{mg} / \mathrm{L}$ for 7-aminoclonazepam. Similarly to the AM, neither PM blood detected the presence of clonazepam. The C:P ratio as well as both PM: AM ratios were equal to 1.0, leading one to believe that PMR occurred. However, the short AMD interval combined with the short PMI (6 hours) may not have been sufficient time to observe a significant decrease in drug concentration. The amount of 7-aminoclonazepam in the PM samples was likely within its expected concentration range at the time of death, suggesting a lack of PMR.

Antemortem blood for CNP 02 had $0.01 \mathrm{mg} / \mathrm{L}$ of clonazepam detected. The sample was collected six hours before death, and had an expected concentration range of $8.3 \times 10^{-3}-9.3 \times 10^{-3} \mathrm{mg} / \mathrm{L}(\mathrm{GC} / \mathrm{ECD}$ limit of detection $=0.001 \mathrm{mg} / \mathrm{L})$. Heart blood was submitted in both gray and red-top collection tubes. Both tubes rendered clonazepam concentrations of $0.03 \mathrm{mg} / \mathrm{L}$. As a result, the PM concentrations were averaged $(0.03 \mathrm{mg} / \mathrm{L})$. The increase of clonazepam in addition to a PM: AM ratio equaling 3.0, resulted in the determination that PMR occurred in this case.

Antemortem blood for CNP 05 was collected 12 hours before death. In this sample, 7-aminoclonazepam had a concentration of $0.17 \mathrm{mg} / \mathrm{L}$ and clonazepam was detected, but at limits below the level of quantitation for the GC/ECD $(0.01 \mathrm{mg} / \mathrm{L})$. Aorta and iliac vein bloods were submitted post autopsy $(\mathrm{PMI}=54$ hours) for analyses. Aorta blood had $0.08 \mathrm{mg} / \mathrm{L}$ of clonazepam and $0.04 \mathrm{mg} / \mathrm{L}$ of 7 -aminoclonzepam. The ILBL had $0.02 \mathrm{mg} / \mathrm{L}$ and $0.47 \mathrm{mg} / \mathrm{L}$ of clonazepam and 7-aminoclonazepam, respectively. Both PM samples had increased levels of clonazepam with respect to the AM sample. 
However, for 7-aminoclonazepam, only the ILBL had a higher level than the AM blood. The PM:AM ratios for 7-aminocloanzepam were 0.23 and 2.8 for the AOBL and ILBL, respectively. Central to peripheral ratios of clonazepam and 7-aminoclonazepam equaled 4.0 and 0.08 , respectively.

The samples analyzed for CNP 05 were all tested on the same day. The PM data showed a clear increase in the concentration of clonazepam with respect to the AM sample. This would suggest that PMR did occur for this drug. However, other observations were made as well. The AOBL returned results that showed an increase in clonazepam and a decrease in 7-aminoclonazepam. So while clonazepam was affected by PMR during the 54 hour PMI, 7-aminoclonazepam levels were reduced as a result of metabolism (AMD interval $=12$ hours). Conversely, there was an increase in both clonazepam and 7-aminoclonzepam concentrations in the iliac vein. This was indicative of PMR influencing both drugs.

The antemortem blood for CNP 03 was collected fourteen hours before death. Clonazepam was detected at a concentration of $0.01 \mathrm{mg} / \mathrm{L}$, while 7-aminoclonazepam was not detected. The expected clonazepam concentration range at the time of death would have been between $0.01-0.01 \mathrm{mg} / \mathrm{L}$ (raw data: $0.006-0.008 \mathrm{mg} / \mathrm{L}$ ). Postmortem mixed and iliac vein bloods were submitted as well (PMI $=19$ hours). Neither PM sample had detectable levels of clonazepam or 7-aminoclonazepam (LC/MS LOD = $0.002 \mathrm{mg} / \mathrm{L}$ ). This would suggest that during the AMD interval clonazepam was effectively eliminated from the body. As a result, PMR was not a factor for this case.

The original analysis of the AM blood for CNP 08 had $0.15 \mathrm{mg} / \mathrm{L}$ of clonazepam and $0.05 \mathrm{mg} / \mathrm{L}$ of 7 -aminoclonazepam detected (AMD interval $=15$ hours). The sample 
was re-tested four months later in a batch which included the cases postmortem (HTBL and ILBL) specimens. The reanalyzed AM blood saw a decrease in clonazepam (0.09 $\mathrm{mg} / \mathrm{L})$ and an increase in 7-aminoclonazepam $(0.17 \mathrm{mg} / \mathrm{L})$, which is likely indicative of clonazepam reduction reaction. When using the data from the re-analysis the expected clonazepam concentration at the time of death ranged between $0.05-0.07 \mathrm{mg} / \mathrm{L}$. The heart blood sample had $0.02 \mathrm{mg} / \mathrm{L}$ of clonazepam and $0.17 \mathrm{mg} / \mathrm{L}$ of 7 -aminoclonazepam. The iliac vein blood had $0.02 \mathrm{mg} / \mathrm{L}$ of clonazepam and $0.10 \mathrm{mg} / \mathrm{L}$ of 7-aminoclonazepam $(\mathrm{PMI}=63$ hours $)$. The PM:AM ratios for clonazepam were 0.22 for both the HTBL and ILBL samples, with a C:P ratio of 1.0. While the PM:AM ratios for 7-aminoclonazepam were 1.0 for HTBL and 0.59 for ILBL, with a C:P ratio of 1.7.

Clonazepam case 08 had conflicting ratio data. Both PM: AM samples suggested a lack of redistribution, while the $\mathrm{C}: \mathrm{P}$ indicated its presence. With a 15 hour AMD interval, one could expect to see a decrease in both drugs. However, only clonazepam levels decreased in the HTBL, which the 7-aminoclonazepam concentration was maintained. Alternatively, this data could be an example of clonazepam reduction. It is not likely that 7-aminoclonazepam would have maintained its concentration under these circumstances. The possibility of clonazepam converting causing the 7aminoclonazepam results to appear unchanged cannot be overlooked. Conversely, the iliac vein data was consistent with metabolism reducing the concentration of both drugs. Therefore, PMR was not a factor for either drug.

The AM and PM samples for clonazepam case 09 were analyzed on the same day as part of a single batch analysis by HPLC/MS. The AM blood, which was collected 66 hours before death, had a 7-aminoclonazepam concentration of $0.04 \mathrm{mg} / \mathrm{L}$. Clonazepam 
was not detected within this sample. Heart and iliac vein bloods were submitted after a 24 hour postmortem interval. Both heart and iliac vein blood samples had 7aminoclonazepam concentrations of $0.04 \mathrm{mg} / \mathrm{L}$. As a result, the PM to AM ratios, as well as the $\mathrm{C}: \mathrm{P}$ ratio equaled 1.0. All of the ratio data suggested that this case was affected by PMR. With such a long AMD interval, it would be expected that the concentration of 7aminoclonzepam would have decreased, not remained steady. Therefore, it does seem that PMR affected the results of this case.

When reviewing the clonazepam data as a whole, it was hard to identify a pattern for when (and if) redistribution would influence the results. For other drugs (e.g. morphine and codeine) reviewed in this manuscript, it was helpful to re-arrange the cases in order of increasing PMI (as opposed to increasing AMD interval). Therefore, the clonazepam data displayed in Table 30 was reorganized to show the cases in order of increasing PMI (Table 31). Six out of the eight cases reviewed had clonazepam present. Of those, only two cases (CNP 02 and 05, 33.3\%) showed signs of postmortem redistribution. This contradicts the apparent $\mathrm{Vd} 1.5-4.4 \mathrm{~L} / \mathrm{kg}$ which is indicative of a moderate level of redistribution. Intertwined within those two cases were CNP 04 and 08 (ordered as 04, 05, 08 and 02). Clonazepam cases 04 and 08 had data consistent with chemical reduction of clonazepam into 7-aminoclonazepam. If one were to only look at case 05 and 02 (both with antemortem to death interval $\leq 12$ hours and PMI $\geq 54$ hours) it would appear that longer PMI may correlate to increased incidences of clonazepam PMR. When focusing specifically on those last 4 cases (PMM28.5 hours, AMD intervals between 10 minutes - 15 hours) it became evident that the role of chemical conversion cannot be overlooked. 
Table 31: Clonazepam cases listed in order of increasing postmortem interval

\begin{tabular}{|c|c|c|c|c|c|c|c|c|}
\hline $\begin{array}{c}\text { PMI } \\
\text { (hours) }\end{array}$ & $\begin{array}{c}\text { AM -Death } \\
\text { (hours) }\end{array}$ & Case \# & $\begin{array}{c}\text { Source } \\
\text { (whole blood) }\end{array}$ & $\begin{array}{c}\text { Concentration } \\
(\mathrm{mg} / \mathrm{L})\end{array}$ & $\begin{array}{c}\mathrm{AM} \\
\text { concentration } \\
(\mathrm{mg} / \mathrm{L}) \\
\end{array}$ & Specimen & $\begin{array}{c}\text { PMR } \\
\text { Clonaz }\end{array}$ & $\begin{array}{c}\text { PMR } \\
\text { 7-amino }\end{array}$ \\
\hline \multirow{2}{*}{6.5} & \multirow{2}{*}{1.5 hours } & \multirow{2}{*}{$\mathrm{CNP} 06^{2}$} & Heart & 0.04 (7amino) & \multirow{2}{*}{0.04 (7amino) } & \multirow{2}{*}{ AMBL } & \multirow{2}{*}{ NDD } & \multirow{2}{*}{ \pm} \\
\hline & & & Iliac & 0.04 (7amino) & & & & \\
\hline \multirow{2}{*}{19} & \multirow{2}{*}{14 hours } & \multirow{2}{*}{ CNP $03^{1}$} & Mixed & NDD & \multirow{2}{*}{0.01 clonaz } & \multirow{2}{*}{ AMBL } & \multirow{2}{*}{-} & \multirow{2}{*}{ NDD } \\
\hline & & & Iliac & NDD & & & & \\
\hline \multirow{2}{*}{24} & \multirow{2}{*}{1 hour } & \multirow{2}{*}{ CNP $07^{2}$} & Heart & 0.05 (7amino) & \multirow{2}{*}{$\begin{array}{c}\text { NDD clonaz } 0.05 \\
\text { (7amino })\end{array}$} & \multirow{2}{*}{ AMBL } & \multirow{2}{*}{-} & \multirow{2}{*}{-} \\
\hline & & & Iliac & 0.06 (7amino) & & & & \\
\hline \multirow{2}{*}{24} & \multirow{2}{*}{66 hours } & \multirow{2}{*}{ CNP $09^{2}$} & Heart & 0.04 (7amino) & \multirow{2}{*}{0.04 (7amino) } & \multirow{2}{*}{ AMBL } & \multirow{2}{*}{ NDD } & \multirow{2}{*}{$\sqrt{ }$} \\
\hline & & & Iliac & 0.04 (7amino) & & & & \\
\hline \multirow{2}{*}{28.5} & \multirow{2}{*}{$10 \mathrm{~min}$} & \multirow{2}{*}{ CNP $04^{1}$} & Iliac & $\begin{array}{c}0.02 \text { clonaz } \\
0.15 \text { (7amino) }\end{array}$ & \multirow{2}{*}{$\begin{array}{c}0.03 \text { clonaz } 0.13 \\
\text { (7amino) }\end{array}$} & \multirow{2}{*}{ AMBL } & \multirow[t]{2}{*}{ - } & \multirow[t]{2}{*}{ - } \\
\hline & & & Iliac & $\begin{array}{c}0.02 \text { clonaz } \\
0.23 \text { (7amino) }\end{array}$ & & & & \\
\hline \multirow{2}{*}{54} & 12 hours & CNP $05^{2}$ & Aorta & $\begin{array}{c}0.08 \text { clonaz } \\
0.04 \text { (7amino) }\end{array}$ & $<$ LOQ clonaz & $\triangle M \mathrm{MI}$ & $\sqrt{2}$ & $\sqrt{ }(\mathrm{II} \mathrm{BI})$ \\
\hline & 12 nours & & Iliac & $\begin{array}{c}0.02 \text { clonaz } \\
0.47 \text { (7amino) }\end{array}$ & 0.17 (7amino) & AIVIDL & & $V($ ILDL $)$ \\
\hline 63 & 15 hours & $C N P O 8^{2}$ & Heart & $\begin{array}{c}0.02 \text { clonaz } \\
0.17 \text { (7amino) }\end{array}$ & 0.09 clonaz 0.17 & $\Delta \mathrm{N}>$ & 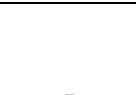 & - \\
\hline 03 & 15 nours & CIVP UO & Iliac & $\begin{array}{c}0.02 \text { clonaz } \\
0.10 \text { (7amino) }\end{array}$ & (7amino) & AIVIDL & - & - \\
\hline 705 & 6 hours & CNP $02^{1}$ & Heart & 0.03 clonaz & 00 & AMBI & $\sqrt{ }$ & NDD \\
\hline T0.5 & o nours & & Heart & 0.03 clonaz & 0.01 & AIVIDL & $v$ & INDD \\
\hline
\end{tabular}

NDD $=$ No Drug Detected

\pm Possible PMR

$\sqrt{ }=$ PMR occurred

- = no PMR 
Six out of the eight cases tested had 7-aminocloanzepam present. Of those six, 1.5 cases $(25 \%)$ had data consistent with the redistribution of 7 -aminoclonazepam. The half percent accounted for CNP 05, where only the ILBL results were consistent with PMR. Not included within the $25 \%$ was case 06 , which was possibly influenced by redistribution, but data could not be definitively qualified. Of the cases that exhibited redistribution, case 05 had AMD interval of 12 hours and a PMI of 54 hours, while case 09 had an AMD of 66 hours with a PMI of 24 hours. With such a broad range for both intervals, it was not possible to correlate PMR with either one. Additionally, neither of the remaining two clonazepam cases (CNP 07 and 08) were influenced by PMR either. Instead, chemical conversion affected their results.

Both CNP 07 and 08 had their AM blood analyzed twice. In both instances, the result was four months from the original test date. The re-test results showed AM conversion of clonazepam to 7-aminoclonazepam for both cases. However in CNP 07 the PM results maintained the same concentration as the AM results, which could be expected here, as the AMD was only one hour. As for CNP 08, the ILBL results were actually reflective of metabolism and elimination occurring during the 15 hour AMD interval. The HTBL sample had results consistent with clonazepam reduction.

Additionally, an interpretive issue arose. Clonazepam cases 06 and 09 each had $\mathrm{AM}$ and PM data (both central and peripheral) return concentrations of $0.4 \mathrm{mg} / \mathrm{L}$. While on the surface it would appear that those two cases should have similar conclusions, it was the AMD intervals, as well as the PM intervals that made the distinction. Clonazepam case 06 had an AMD of 1.5 hours and a PMI of 6.5 hours. Neither interval appeared to be a sufficient amount of time to allow redistribution to occur. It was more 
likely that a slight reduction in drug concentration occurred after the AM collection and the short PMI was not enough time to allow for the release and distribution of the drug postmortem. Conversely, CNP 09 had an AMD interval of 66 hours, which according to the elimination half-life (19 - 60 hours) would have been enough time to see a $50 \%$ decrease in drug concentration. Instead of a decrease, the PM samples appeared to maintain their drug concentrations. It is likely that during the 24 hour PMI, the drug was released and redistributed.

In all, clonazepam is a drug that may not be easily interpreted postmortem. The effects of pharmacokinetics, PMR and time intervals could not be clearly defined. The data collected here was a stark contradiction to what would be expected when considering the apparent volume of distribution of the drug. In fact, more cases were influenced by chemical conversion than redistribution. As a result, anytime clonazepam needs to be measured, it would be advisable to monitor the levels of 7-aminoclonazepam as well. Quantitative results for both drugs may be helpful when trying to determine the meaning of the PM results. If AM blood is available, it should be tested immediately, as chemical conversion can affect the results of that specimen as well.

\subsection{Microbial Study}

The main purpose of the microbial study was to determine if microbial growth could contribute to changes in drug concentration. This was assessed in two ways. First, it was important to know if microbes could use drugs as a food source. Second, but of 
equal importance, was to determine if the addition of sodium fluoride (anti-microbial agent) to the blood samples was a sufficient means of halting bacterial growth.

\subsubsection{Codeine and Hydrocodone as a Food Source for Bacteria}

A 96-well MT plate (Figure 5) was set-up as described in section 4.5.4 Assessing the Usefulness of Codeine and Hydrocodone as a Food Source for Bacteria. On day 0 the initial reading was taken. At that time the cells were inoculated with the bacteria, as well as the drug standards at concentrations of 100,200 and $600 \mathrm{ng} / \mathrm{mL}$. The amount of growth was monitored for 6 consecutive days thereafter.

The codeine data is displayed in Chart 12. On average the Pseudomonas growth patterns exceeded those of E. coli by three times or more. The Pseudomonas bacteria mixed with the $100 \mathrm{ng} / \mathrm{mL}$ standard saw the most growth, while the $600 \mathrm{ng} / \mathrm{mL}$ standard saw the least. Conversely, the opposite was true for E. coli as the $600 \mathrm{ng} / \mathrm{mL}$ grew the best, with the $100 \mathrm{ng} / \mathrm{mL}$ growing the least. Despite the growth discrepancy observed among the inter-bacterial data, statistical analysis proved that the intra-bacteria growth patterns were not significantly different throughout the observation period.

Chart 13 displays the results of the hydrocodone containing cells. The growth pattern observed among this data set was similar to that of the codeine results. Again, Pseudomonas had at least three times the growth of E. coli. However, throughout the seven day period, it was E. coli that had the least amount of variation. The best growth was observed when Pseudomonas was combined with the $200 \mathrm{ng} / \mathrm{mL}$ standard. Conversely, E. coli grew the best when mixed with the $100 \mathrm{ng} / \mathrm{mL}$ standard. For both data sets, the $600 \mathrm{ng} / \mathrm{mL}$ standards saw the least amount of growth. 
Overall, the drug standards that contained Pseudomonas outgrew the cells containing E. coli. A subsequent photometer reading of the microbial solutions used to inoculate the plates was taken. The results determined that the density of Pseudomonas (1.5) was approximately $87 \%$ higher than that of $E$. coli $(0.23)$. As a result it would be expected that Pseudomonas would outperform E. coli. Also working to the advantage of Pseudomonas is that it is amenable to a multitude of environments. While it is known to grow in locations such as skin and soil, it is not a microbe commonly associated with the human decomposition process. It was included in this study more as a gauge to determine if it would be possible for bacteria to use the carbon structures of drugs as a source of food. Conversely, Escherichia coli are known to be present within the intestines. As tissues breakdown, the bacteria can release and spread throughout the body. Therefore, the way E. coli responded to the project was of special interest. So while, Pseudomonas responded better to the growth conditions, both Pseudomonas and E. coli proved this was possible for bacteria to be sustained by nothing more than the carbon structure of drugs.

\subsubsection{Aerobic and Anaerobic Study}

In order to assess the ability of sodium fluoride to halt microbial growth upon sample collection, antemortem and postmortem bloods containing the agent were inoculated onto nutrient agar plates. The AMBL was collected and stored in a GT tube until the time of testing. The PM blood (collected in both red-top and gray-top tubes) came from a single decomposing cadaver. The liquefied blood from both tubes, as well as the dried blood accumulating around the lid of the RT, was inoculated. The plates 
Chart 12: Pseudomonas and E. coli Growth Patterns When Using Codeine as a Food Source

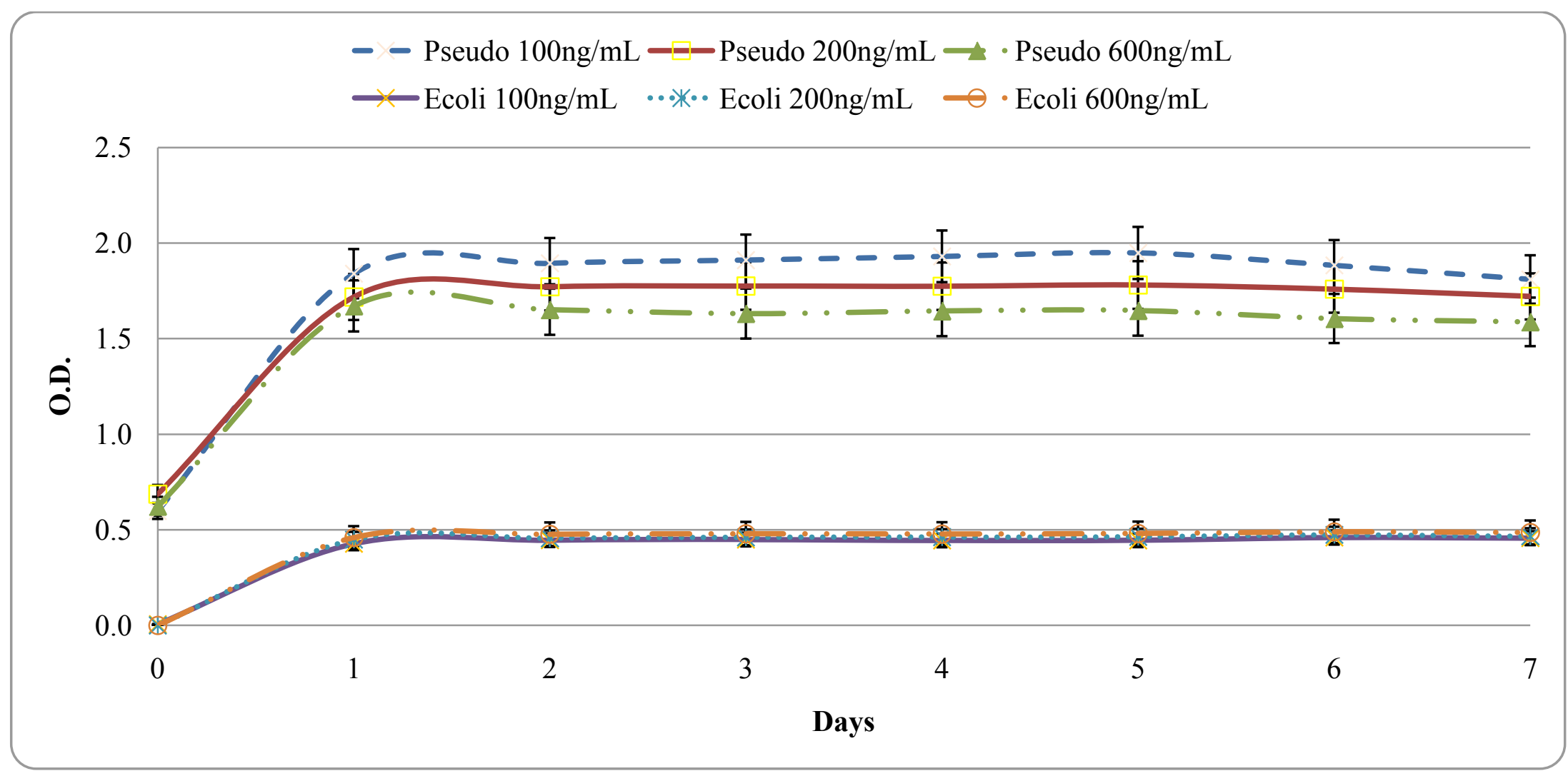


Chart 13: Pseudomonas and E. coli Growth Patterns When Using Hydrocodone as a Food Source

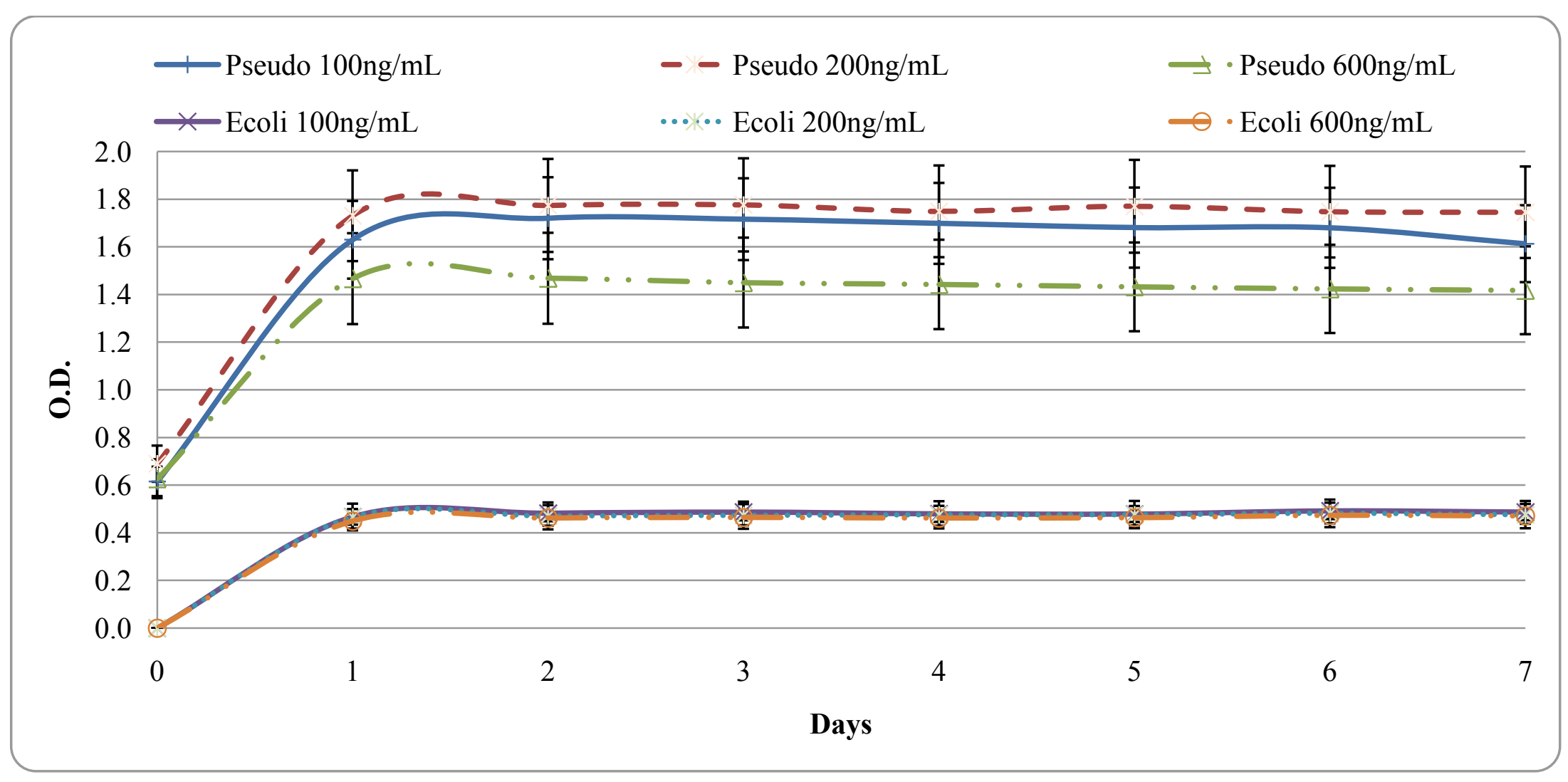


were prepared in duplicate, with one set stored under aerobic conditions and another in an anaerobic environment. Both sets were maintained for 11 days at $17{ }^{\circ} \mathrm{C}$. The results for the aerobic and anaerobic studies can be seen in Table 32 and Table 33, respectively.

The initial results of the aerobic study saw growth on one of the RT chest blood replicates, but not the other. As a result, the blood from that tube was retested in duplicate. The re-analysis did not show any growth on either plate. While the agar and the plastic loops (used for both the original and re-test experiments) were sterile, the pipette tips (used to spike the $75 \mu \mathrm{L}$ of blood onto the plates) were not. In all likelihood, the non-sterile tip, used to spike the original plate that showed growth, was contaminated. The AM and PM red-top, as well as the PM gray-top, showed no signs of growth when stored in an anaerobic environment. However, both replicates of the dried RT blood did. Since both replicates grew, this was not believed to be a contamination issue. In order to determine what type of anaerobe was growing, the plates were sent for microbial identification at the International Forensic Research Institute's DNA profiling laboratory on the campus of Florida International University. The identification results are still pending.

Overall, it was determined that under aerobic conditions sodium fluoride is a suitable additive for the prevention of microbial growth. When properly prepared, neither AM or PM samples saw any growth. This is of the utmost importance, as once the blood is collected it is maintained in an aerobic environment. Conversely, growth was detected in the anaerobic experiment. The anaerobic experiment, however, was not without flaws, as the blood used had already been exposed to oxygen for several 
Table 32: Growth Activity of Preserved Blood Stored in an Aerobic Environment

\begin{tabular}{|ccccc|}
\hline Sample Type & Source & Tube & Growth & Action \\
\hline AM & AMBL & GT & X & N/A \\
\hline PM & CHBL & RT & $\sqrt{ } 1$ & Re-streaked \\
\hline PM & CHBL & GT & X & N/A \\
\hline PM & CHBL & RT dried blood & X & N/A \\
\hline
\end{tabular}

Table 33: Growth Activity of Preserved Blood Stored in an Anaerobic Environment

\begin{tabular}{|ccccc|}
\hline Sample Type & Source & Tube & Growth & Action \\
\hline AM & AMBL & GT & X & N/A \\
\hline PM & CHBL & RT & X & N/A \\
\hline PM & CHBL & GT & X & N/A \\
\hline PM & CHBL & RT dried blood & $\sqrt{ }$ & sent for identification \\
\hline
\end{tabular}

$$
\begin{gathered}
\mathrm{X}=\text { no growth } \\
\sqrt{ }=\text { growth } \\
\sqrt{ }{ }^{1}=\text { growth on one replicate only } \\
\text { N/A }=\text { not applicable }
\end{gathered}
$$


months prior to testing. This would result in the death of the strict anaerobes, but the possible survival of the facultative anaerobes (those that can survive in either oxygen-rich or oxygen-depleted environments). Bearing that in mind, the only growth detected was on the dried blood sample. Out of the samples tested, it was the only one with an obvious depletion of oxygen. Therefore, any organism that could survive those conditions would likely thrive in an oxygen-free environment.

This portion of the experiment showed that under the current storage conditions, continued growth of bacteria in the collected blood samples is not a concern. However, the effect of bacterial growth in the body prior to sample collection may impact drug concentrations.

\subsubsection{Cumulative Microbial Results}

The combination of the MT plate and agar plate experiments rendered some results of interest. Drugs can in fact be used as a food source for bacteria. However, as shown in the agar plate experiment, once the blood is collected and preserved (in an aerobic environment) bacteria, including $E$. coli, were no longer viable. On the other hand, under anaerobic conditions some bacteria (potentially facultative anaerobes) did grow. This was not deemed a problem for two reasons. First, once collected, all of the samples are stored aerobically, not anaerobically. Second, the only growth seen in the anaerobic study came from a dried blood sample. When drug identification and quantitation are performed, fluid blood, not dried blood, is used. The results of the anaerobic study showed no growth on any of the fluid blood samples. The presence of those bacteria should not negatively influence the results. 


\subsection{Stability Study}

Hydrocodone and codeine standards, prepared in dilute phosphate buffered saline solution, were spiked in whole blood to give a final concentration of $200 \mathrm{ng} / \mathrm{mL}$. Analytical analysis conducted by GC/MS/MS quantitatively monitored codeine and its metabolite morphine, while hydrocodone and its metabolite hydromorphone were analyzed by GC/ SIM-MS. The initial analysis of both drugs was conducted by GC/MS/MS; however, an interference causing the ion suppression of the hydrocodone-d3 ion (288) resulted in the inability to detect the internal standard ion. It was decided that the Opiate II quantitative method $(4.4 .2$ (b) Opiate II by GC/MS (SIM)) could be used alternatively. In that method, hydrocodone-d3 was still the internal standard; however, the 331 ion was monitored instead. For both methods, the metabolites of the parent drugs were also monitored, as it was imperative to determine if any concentration changes could be attributed to chemical conversion. Initial analysis of codeine was conducted on the same day of the spike. As a result of the interferent, hydrocodone's initial analysis was delayed by three days. For both drugs however, re-analyses were performed 30 and 60 days later. In between testing dates, the blood samples were refrigerated at $2{ }^{\circ} \mathrm{C}$. The results are displayed in Table 34.

Codeine, a drug evaluated in section 6.1.1 Heroin, Morphine and Codeine, had an error rate (established during research testing) of 10\%. Hydrocodone was not assessed in any other part of this study. Therefore, in order to determine the amount of error associated with the drug, the standard deviation (2.988) and concentration mean $(22 \mathrm{ng} / \mathrm{mL})$ of the controls collected as part of routine analysis were calculated. When using Equation 5 the $\mathrm{CV}$ was determined to be 13\%. Any concentrations which fell 
within the established limits of error were considered statistically similar, while those that exceeded the limits were not.

Codeine had an initial reading of $191 \mathrm{ng} / \mathrm{mL}$ in the RT and $197 \mathrm{ng} / \mathrm{mL}$ in the GT. Both concentrations were similar to each other, as well as being similar to the $200 \mathrm{ng} / \mathrm{mL}$ spiked concentration. At the second reading, concentration discrepancies began to emerge in the RT tube. Its blood concentration decreased to $179 \mathrm{ng} / \mathrm{mL}$. While this level was statistically similar to the previous concentration reading, it exceeded the limits of the $\mathrm{CV}( \pm 10 \%)$, thereby making it significantly different from the originally spiked concentration. Conversely, the amount of drug in the GT tube $(183 \mathrm{ng} / \mathrm{mL})$ was similar to both the spiked and the initial concentrations. The third and final reading again showed a decrease in codeine concentrations. This time, both RT and GT (166 ng/mL each) levels were statistically lower than both the spiked amount and the initial reading.

Table 34: Results of the 60 Day Drug Stability Study of Refrigerated Blood

\begin{tabular}{|c|c|c|c|c|c|}
\hline Analyte & $\begin{array}{c}\text { Spiked } \\
\text { Concentration }\end{array}$ & Tube & $\begin{array}{c}\text { Initial } \\
\text { Reading } \\
(\mathrm{ng} / \mathrm{mL})\end{array}$ & $\begin{array}{c}\text { 30 Day } \\
\text { Reading } \\
(\mathrm{ng} / \mathrm{mL})\end{array}$ & $\begin{array}{c}60 \text { Day } \\
\text { Reading } \\
(\mathrm{ng} / \mathrm{mL})\end{array}$ \\
\hline \multirow{2}{*}{ Codeine } & $200 \mathrm{ng} / \mathrm{mL}$ & RT & 191 & 179 & 166 \\
\cline { 3 - 6 } & GT & 197 & 183 & 166 \\
\hline $\begin{array}{c}\text { Morphine } \\
\text { (metabolite) }\end{array}$ & N/A & RT & not detected & not detected & not detected \\
\cline { 3 - 6 } Hydrocodone & \multirow{2}{*}{$200 \mathrm{ng} / \mathrm{mL}$} & RT & not detected & not detected & not detected \\
\cline { 3 - 6 } & GT & 131 & 131 & 130 \\
\hline $\begin{array}{c}\text { Hydromorphone } \\
\text { (metabolite) }\end{array}$ & \multirow{2}{*}{$\mathrm{N} / \mathrm{A}$} & $\mathrm{RT}$ & not detected & not detected & not detected \\
\cline { 3 - 7 } & & GT & not detected & not detected & not detected \\
\hline
\end{tabular}

Red-top tube $=\mathrm{RT}$

Gray-top tube $=$ GT 
The steady decline in the codeine concentration for both tubes indicated that under the current storage conditions, codeine may not be stable. The lack of morphine detection would suggest that while chemical conversion did not seem to be a factor, drug degradation should still be considered. This conclusion was in direct contrast to the results found in both the Holmgren (45) and Peters (44) studies. They each determined that codeine was stable in storage for a period of at least one year. Their blood samples were frozen at temperatures of $-20{ }^{\circ} \mathrm{C}$ and $-18{ }^{\circ} \mathrm{C}$, respectively. Based on this information, it would appear that drug preservation of codeine is much more reliable when the sample is frozen, not refrigerated.

After the collection of the hydrocodone data, the results were determined to be skewed. As previously mentioned, hydrocodone had to be analyzed by GC/SIM-MS as a result of an internal standard interference observed in the GC/MS/MS data. Upon initial analysis (using the SIM method) a prominent internal standard peak was detected and the instrument switch was made. The initial reading saw a concentration decrease of $65 \%$ in the RT $(131 \mathrm{ng} / \mathrm{mL})$ and $63 \%$ in the GT $(126 \mathrm{ng} / \mathrm{mL})$ tubes. At the time, the reason for the significant concentration decrease was not known. The analyses continued 30 days $(\mathrm{RT}=131 \mathrm{ng} / \mathrm{mL} ; \mathrm{GT}=127 \mathrm{ng} / \mathrm{mL})$ and 60 days $(\mathrm{RT}=130 \mathrm{ng} / \mathrm{mL} ; \mathrm{GT}=126 \mathrm{ng} / \mathrm{mL})$ later. Although significantly decreased from the spiked amount $(200 \mathrm{ng} / \mathrm{mL})$, the monthly readings were consistent to each other, regardless of sample tube.

After careful review of the hydrocodone standard preparation procedure and the resultant analytical findings, it was determined that the internal standard was again causing a problem. While the MS/MS method saw ion suppression of the internal standard, the SIM method saw ion enhancement. The internal standard for the sample 
produced area responses that ranged from $11-18 \%$ higher than the calibrators on any given run. The increased internal standard resulted in decreased sample concentration, as concentrations are calculated based on an area response ratio between the sample and internal standard. In theory, if the problem had been caught in the beginning the SIM method still could have been used. However, the sample should have been extracted using the method of standard additions.

The method of standard additions is used to resolve problems associated with matrix effects (e.g. ion suppression or enhancement). To account for this problem, the sample itself should have been spiked with known drug concentrations to produce a calibration curve. The sample concentration could then have been calculated against that curve. While this would have fixed the miscalculation, in actuality the volume of the sample available for testing would not have been sufficient to allow for three separate methods of addition calculations. Each batch, which consisted of a blank, a five-point calibration curve, four controls (low and high concentrations in duplicate) and sample extraction in duplicate, would have required a minimum of $12 \mathrm{~mL}$ of sample. The graytop tubes used hold $10 \mathrm{~mL}$ each ( 2 tubes total) and there was $30 \mathrm{~mL}$ in the red-top. In addition, sample blood for the GC/MS/MS method was needed as well.

In the absence of the standard addition data, the internal standard responses were corrected for in each batch. While this is a practice never used at the MDME- toxicology laboratory, using a corrective calculation in this instance is the resource available to the researcher in the absence of additional blood. It was important to use this particular blood sample because it was the same blood used in the microbial study. Therefore, the results could be better compared. 
In order to correct for the internal standard bias, the average internal standard response was calculated for the 5-point curve. The internal standard response for the RT and GT tubes were then averaged based on their respective collection tube, followed by an average calculation for the sample (RT and GT) tubes. The percent difference between the calibration curve and sample internal standard responses were calculated. For example, in one batch the red-top and gray-top internal standard responses exceeded the curve response by $11 \%$ and $16 \%$, respectively. The internal standard response of the case was decreased by its appropriate percentage based on the sample tube. After this, the hydrocodone concentrations were recalculated using the adjusted internal standard responses. Applying this correction, resulted in sample concentrations ranging from 151 to $164 \mathrm{ng} / \mathrm{mL}$. When comparing these results to the method CV (13\%), all of the recalculated values remained statistically similar to each other regardless of sample tube. Although the concentrations were still significantly lower than the spiked concentration, the adjusted concentrations were between $18-25 \%$ lower. This was a vast improvement over the non-adjusted calculations which resulted in discrepancies ranging from $63-$ $65 \%$ lower.

In all, this experiment showed that refrigerated storage may not be the best option for analyte preservation of some drugs (specifically codeine). However, since the graytop tubes are made of glass, freezing them may not be a wise alternative either. In lieu of changing the type of collection tube used, it would be in the best interest of the analyst(s) to conduct quantitative analyses relatively quickly, as concentration levels may change in storage. This instability may not affect all drugs, as was seen here with hydrocodone. 
While hydrocodone levels were consistently lower than the spiked amount, the concentration remained constant over the three month testing period. In addition, while this study was not intended to account for the difficulties that matrix effects can present, their influence on this drug was evident. This is an issue that can present itself at anytime. It is especially important to consider when testing postmortem biological samples. In this instance, the sample was from a decomposing cadaver. In such a situation, multiple biological processes are occurring and interferences may result. This issue did not present itself in other samples used for this study. However, none of the other samples were taken from a cadaver in such a state of decomposition. Regardless, in real-life instances the role of matrix effects should be considered when reviewing the analytical data.

\subsection{Conclusions:}

The goals of this project were to 1) evaluate the differences in drug concentrations between antemortem and postmortem specimens, 2) determine how the presence of microbes influence drug concentrations, 3) determine the stability of drugs in whole blood and 4) define an interpretive framework to aid in the interpretation of postmortem drug concentrations in whole blood. In Chapter 6: Results and Discussion, the first three goals were discussed in great detail.

\subsection{Microbes and Stability: Potential Influences on Postmortem Concentrations}

In all, it was determined that in the absence of a preservative, both Escherichia coli and Pseudomonas aeruginosa can use drugs (specifically codeine and hydrocodone) 
as a carbon source for food. It is however important to keep in mind that the drugs analyzed for this study were preserved in tubes that contained the anti-microbial agent sodium fluoride. After inoculating preserved blood samples, it was determined that under the current storage conditions, sodium fluoride effectively eliminated microbial activity.

The same PM blood and drug standards used for the microbial experiment were also used for the stability study. Aliquots of the blood (stored in both gray-top vacutainer and red-top polypropylene tubes) were spiked with both codeine and hydrocodone to give a final concentration of $200 \mathrm{ng} / \mathrm{mL}$ each. The blood was analyzed by GC/SIM-MS and the GC/MS/MS monthly for a period of 60 days. Included in the analytical method were morphine and hydromorphone, the metabolites of codeine and hydrocodone, respectively. By monitoring both parent and metabolite concentrations the role of chemical conversion could be considered. The results indicated the refrigerated storage can effectively maintain hydrocodone concentrations. Unfortunately, the same was not true for codeine, which steadily declined in concentration throughout the study. However, when combining the microbial and stability results it was determined that instances of concentration instability were not a result of microbial degradation. In addition, at least for these two drugs, chemical conversion did not contribute either. For a future study, the role of storage temperature and photo-degradation should be considered, as those two factors may have influenced the codeine results.

The data generated from the microbial and stability experiments played a fundamental role in achieving the first goal of the experiment, which was to evaluate the differences in drug concentrations observed in antemortem and postmortem samples. When differences were identified, it was important to determine the cause of the 
discrepancy. Some drugs (e.g. diphenhydramine, morphine and codeine) produced data that was overwhelmingly consistent with being influenced by postmortem redistribution. This was apparent when the postmortem results were consistently greater than the drug levels in their respective antemortem samples. In contrast, other drugs like midazolam were efficiently metabolized and eliminated from the body. Unfortunately, not all of the data collected was as straight forward.

\subsection{Interpretive Guidelines}

The combined information gained from the first three goals was used to achieve the fourth goal, which was to develop an interpretative framework for the postmortem results. Upon examining the data, it quickly became apparent that strict interpretative rules would not work. Just as decomposition is a dynamic process that can vary drastically based on a number of different factors, the interpretation of postmortem results can too. Even analytes within the same drug class do not necessarily behave similarly. In lieu of developing strict guidelines, a list of important interpretative considerations was developed.

According to its apparent volume of distribution $(3-4 \mathrm{~L} / \mathrm{kg})$, diphenhydramine is a drug that has a propensity for postmortem redistribution. The experimental data collected for this study was in agreement with that. The study results indicated that PMR can begin as quickly as 11 hours after death. The results consistently indicated that PM diphenhydramine levels will likely be significantly higher than their AM counterparts. In addition, while it is often believed that peripheral blood concentrations most closely resemble drug levels at the time of death, the data generated here contradicted that, 
indicating that there was no direct correlation between PM peripheral and AM blood concentrations. In actuality, PM diphenhydramine concentrations (whether central or peripheral) typically will greatly exceed both their AM and expected concentrations levels at the time of death. As a result, when reviewing diphenhydramine postmortem results, it would be advisable to bear in mind that the concentrations obtained are likely elevated.

The benzodiazepines alprazolam, midazolam, diazepam and clonazepam are a class of drugs known for their central nervous system depressant and muscle relaxant properties. Although pharmacologically related, it should not be assumed that they all respond similarly in a postmortem environment. Alprazolam data suggested that the drug was subject to PMR when the combination of a short antemortem to death interval $(\leq 40 \mathrm{~min})$ was coupled with a long postmortem interval ( $\geq 45.5$ hours). The results obtained in this study determined that any other variation of those two intervals likely would not result in redistribution. These results were reasonable, as its apparent volume of distribution $\left(\mathrm{V}_{\mathrm{d}}=0.9-1.3 \mathrm{~L} / \mathrm{kg}\right)$ did not reflect a strong tendency for redistribution. In addition, when comparing postmortem central to peripheral concentrations, $71 \%$ of the cases showed no significant difference between the two sources. In most instances, quantitative analysis could be conducted on either PM specimen. That is not to say that the PM results would be equivalent to the expected concentration range at the time of death. In fact only $25 \%$ of the PM data collected fell within its expected range at the time of death. The remaining $75 \%$ were either above or below the calculated expectation. In all, although alprazolam is not likely to redistribute, a skew of the PM results may still exist. 
Midazolam is a benzodiazepine which rapidly metabolizes from the body $\left(\mathrm{t}_{1 / 2}=1\right.$ 4 hours). While its apparent volume of distribution $(1.0-2.5 \mathrm{~L} / \mathrm{kg})$ was indicative of a drug that could effectively undergo postmortem redistribution, the data collected refuted this. In $100 \%$ of the cases no signs of redistribution could be identified, regardless of AMD (range: 16 min -48 hours) or PM (10 - 37 hours) intervals. In all instances, it appeared as though metabolism and elimination effectively decreased (and in many instances removed) the drug from the body prior to death.

The benzodiazepine diazepam was monitored along with its pharmacologically active metabolite nordiazepam. With elimination half-lives of $21-37$ hours and $50-99$ hours for diazepam and nordiazepam, respectively, both drugs are considered to have a long-lasting effect. When comparing their postmortem concentrations to their antemortem's, there was an expectation that signs of chemical conversion between the two would be evident. However, that was not the case. In most instances when comparing PM to AM concentrations, both parent and metabolite would move in parallel to each other, meaning they would both either increase or decrease within the same case. This could be reflective of diazepam's stability in storage, as drug instability may have led to more instances of opposing concentrations shifts between the parent and metabolite. Their similar concentration shifts did not negate the influence of PMR on either drug, as $50 \%$ of the data (for both drugs) was indicative of PMR. The remaining $50 \%$ of the cases had PM concentrations either within or below their expected concentrations at the time of death. When comparing central to peripheral data in most instances either specimen could be used to quantify diazepam, however peripheral nordiazepam concentrations were typically lower. Since diazepam and nordiazepam are 
monitored together in most labs, peripheral blood would be the preferred source for quantitative analyses. However, for both drugs, it should not be expected that peripheral results are reflective of drug concentrations at the time of death, as only $17 \%$ of diazepam and $0 \%$ of nordiazepam results were within their calculated concentration ranges.

Clonazepam was a drug that presented many challenges. It is classified as a longacting benzodiazepine ( $t_{1 / 2}=19-60$ hours), but a prolonged effect in a living person, did not equate to drug stability in a postmortem specimen. Clonazepam, monitored here with its pharmacologically inactive metabolite, 7-aminoclonazepam, was largely susceptible to chemical reduction in storage. As a result, the levels of clonazepam would reduce, while 7-aminoclonazepam levels would increase. This was of particular interest because clonazepam's apparent volume of distribution $(1.5-4.4 \mathrm{~L} / \mathrm{kg})$ suggested that the drug was prone to PMR. The results of this study determined that only $37 \%$ of the cases were susceptible to redistribution. When examining the results, it became rather difficult to identify a predictable pattern for when and how drug concentrations would change. As a result, in instances of quantitative analysis, interpretation should be done with caution. In an effort to reduce these PM concentration concerns, it may seem like a good idea to only analyze the AM blood for this particular drug. However, the reanalysis of antemortem blood samples $(\mathrm{n}=2)$ determined that clonazepam concentrations can decrease as early as four months in storage $\left(2{ }^{\circ} \mathrm{C}\right)$. Although two samples do not equate to a significant populations size, the data did suggest that when clonazepam levels are a concern, the samples should be analyzed as close to collection as possible. In addition the 7aminoclonazepam concentration should be monitored as well. 
The synthetically derived opioid methadone, as well as the naturally occurring opiates morphine and codeine were also examined for this study. In keeping with its apparent volume of distribution $(4-5 \mathrm{~L} / \mathrm{kg})$, methadone consistently produced data that was influenced by PMR, with the impact being greater on the central sourced blood. The postmortem results (regardless of source) were routinely greater than both the expected concentration ranges at the time of death, as well as the AM concentrations. The data indicated that PMR would occur for this drug regardless of AMD (45 min - 72 hours) or PM intervals (16 - 54 hours), as their ranges varied greatly. When interpreting PM methadone levels, it would be advisable to consider the drug concentrations elevated.

Because of the pharmacological similarities, morphine and codeine are two drugs that are not commonly prescribed together. However, they are often detected together, as morphine is a major metabolite of codeine. In addition morphine (as a metabolite) and codeine (as a by-product) are also produced as a result of heroin metabolism. It was only logical to review their results together. In keeping with their apparent volumes of distribution, both morphine $\left(\mathrm{V}_{\mathrm{d}}=2-5 \mathrm{~L} / \mathrm{kg}\right)$ and codeine $\left(\mathrm{V}_{\mathrm{d}}=3.5 \mathrm{~L} / \mathrm{kg}\right)$ exhibited a strong predisposition for PMR. By re-arranging the data in order of increasing postmortem interval, it became very clear that both drugs exhibited increased incidences of redistribution with longer PM intervals. Conversely, the influence of the antemortem to death interval (range: 9 minutes -24 hours) was negligible. 


\subsection{Additional Considerations}

The role of chemical conversion between codeine and morphine was a minor issue, as only $15 \%$ of the cases were affected. When comparing PM central and peripheral results, it became apparent that in most instances the extent of PMR was site dependent, with the central source seeing a greater affect. However, factors such as the location of intravenous injection (in the case of heroin abuse) should not be overlooked as this can alter the redistribution effect. Overall the peripheral sourced blood had lower drug concentrations, only 1 morphine case had a level within its expected concentration range at the time of death. The interpretation of both morphine and codeine in postmortem specimens should be done with caution.

Finally, throughout the data collection process, central: peripheral, as well as postmortem: antemortem ratio results were being collected and compared. The ratio results conflicted in $54 \%$ of the cases. When comparing both ratios, to the final determinations made by the researcher, it was the PM: AM ratio that was most often in agreement. As a result, it was determined that while the C:P ratio could be a great tool in determining whether or not redistribution occurred in a predicted fashion (e.g. redistribution was more pronounced in the central sourced blood), it may not be the most reliable way of evaluating whether or not PMR actually happened.

\subsection{Final Thoughts and Future Work}

Overall, the goals for this project were met. While in some instances definitive conclusions could not be drawn, the body of work in its entirety is an examination of the 
various issues that plague a postmortem toxicologist. A drug concentration alone carries no weight. The understanding of what it means, its application and whether or not its presence influenced the death of someone is of the utmost importance. This study investigated theories and ideas common to toxicological professionals. While some concepts were reinforced, others were not. It would be advisable that future experiments continue to examine the importance of ratio data (e.g. central:peripheral and postmortem:antemortem), as well as the affect clonazepam stability has on postmortem interpretations. Additionally, further studies concerning the impact of microbial activity on drug concentrations prior to sample collection are needed. By examining factors such as temperature, the addition of biological carbon sources (mixed with drug carbon sources) and environment (e.g. aerobic or anaerobic), the true impact bacteria have on toxicological findings may be better identified. 


\section{LIST OF REFERENCES}

1. Miami-Dade County-Medical Examiner's Department. [Online] [Cited: October 26, 2008.] http://www.miamidade.gov/medexam accessed on 10.26.08.

2. State of Florida - Senate. [Online] [Cited: October 26, 2008.]

http://www.flsenate.gov/Statutes/index.cfm?App_mode=Display_Statute\&URL $=$ Ch0406 /ch0406.htm/10.26.08.

3. Miami-Dade County - Toxicology. [Online] [Cited: October 26, 2008.]

http://www.miamidade.gov/medexam/toxicology.

4. Accreditaion Board of Forensic Toxicologists (ABFT). [Online] [Cited: October 26, 2008.] http://www.abft.org .

5. Society of Forensic Toxicologists (SOFT). [Online] [Cited: October 26, 2008.] http://www.soft-tox.org/default.aspx?pn=Introduction\&sp=Introduction .

6. Brunton, Laurence, [ed.]. Goodman and Gillman's The Pharmacological Basis of Therapeutics. 11th. s.1. : McGraw-Hill medical publishing division, 2006. p. 1744.

7. Brunton, Laurence, [ed.]. Goodman and Gillman's The Pharmacological Basis of Therapeutics. s.1. : McGraw-Hill Medical Publishing Division, 2006. pp. 33-37.

8. McKee, T and McKee, J. Biochemistry An Introduction. 2. s.1. : WCB McGraw-Hill, 1999. p. 241.pg. 241.

9. McKee, T. and McKee, J. Biochemistry An Introduction. 2. s.1. : WCB McGraw-Hill, 1999. p. 240.

10. Brunton, Laurence, [ed.]. Goodman and Gillman's The Pharmacological Basis of Therapeutics. 11. s.1. : McGraw-Hill medical publishing division, 2006. p. 7.

11. Trevor, A., Katzung, B., Masters, S. Katzung \& Trevors Pharmacology Examination Board Review. 8. New York : McGraw Hill Medical, 2008. p. 21.

12. Cornell University - Vetenary Medicine. [Online] [Cited: November 26, 2008.] http://web.vet.cornell.edu/public/pharmacokinetics/sitesDT/phase1vs2.html.

13. Creighton University. [Online] [Cited: November 26, 2008.]

http://pharmacyonline.creighton.edu/pha443/pdf/pkin03.pdf.

14. Medical Terminology - Online. [Online] [Cited: November 26, 2008.] http://www.medterms.com. 
15. Beyond the Grave - Understanding Human Decomposition. Vass, Arpad. s.1. : Microbology Today, 2001, Vol. 28, pp. 130-132.

16. Sledzik, P. Forensic Taphonomy: Postmortem Decomposition and Decay. Forensic Osteology. p. C. 5.

17. Missouri - Case Studies in Forensic Entomology. [Online] [Cited: November 26, 2008.] http://www.missouri.edu/ agwww/entomology/casestudies.html.

18. Farmer's Almanac Online. [Online] [Cited: December 29, 2008.] almanac.com/weatherhistory .

19. Weather online. [Online] [Cited: December 29, 2008.] almanac.com/weatherhistory .

20. Mechanisms underlying postmortem redistribution of drugs: A review. PelissierAlicot, A. and Gaulier, J, et al. s.l. : Journal of Analytical Toxicology, 2003, Vol. 27, pp. 533-543.

21. Postmortem toxicology of drugs of abuse. Drummer, O. 2004, Forensic Science International, Vol. 142, pp. 101-113.

22. Estimating antemortem drug concentrations from postmortem blood samples: the influence of postmortem redistribution. Cook, D., Braithwaite, R. and Hale, K. 53, s.1. : J. Clin Pathol, 2000, pp. 282-285.

23. Goodman and Gillman's The Pharmacological Basis of Therapeutics. 9. 1996.

24. Hearn, W.L. Toxicology: Interpretation of results. [ed.] Jay A. Siegel. s.1. : Encyclopedia in Forensic Sciences, 2000. pp. 1391-1397.

25. Hepler, B. and Isenchmid, D. Specimen selection, collection, preservation and security. [ed.] S. Karch. Drug Abuse Handbook. s.1. : CRC Press, 1998, 12.2, pp. 876880. various biological specimens.

26. The extent of postmortem drug redistribution in a rat model. Hilberg, T., et al. 44, s.1. : J Forensic Sci, 1999, Vol. 5, pp. 956-962.

27. Hepler, B. and Isenschmid, D. Interpretation of postmortem drug levels. [ed.] S. Karch. Drug Abuse Handbook. s.1. : CRC Press, 1998, p. 881. bile, vitreous humor.

28. Interpreting results of ethanol analysis in postmortem specimens: A review of the literature. Kugelberg, F and Jones, A. 165, s.1. : For Sci Int, 2007, pp. 10-29.

29. Jones, G. Interpretation of postmortem drug levels. [ed.] S. Karch. Drug Abuse Handbook. s.1. : CRC Press, 1998, pp. 973-974. 
30. Baselt, R., [ed.]. Diposition of toxic chemicals and drugs in man. 7th. s.1. :

Biomedical Publications, 2004. pp. 532-534. Heroin.

31. Recent discoveries in pharmacokinetics of drugs of abuse. Cone, E. s.1. : Toxicology Letters, 1998, Vols. 102-103, pp. 97-101.

32. Free Oxycodone Concentrations in 67 Postmortem Cases from the Hennepin County Medical Examiner's Office. Thompson, J. s.1. : JAT, 2008, Vol. 32, pp. 673-679.

33. Benzodiazepines- Effects on human performance and behavior. Drummer, O. 1, s.1. : Forensic Science International, January 2002, Vol. 14.

34. Effectiveness of benzodiazepines - do they work or not? Lader, M. 8, s.1. : Expert Reviews Neurotherapeutics, 2008, Vol. 8, pp. 1189-1191.

35. The diagnosis and management of benzodiazepine dependence. Ashton, H. s.1. : Current Opinion in Psychiatry, 2005, Vol. 18, pp. 249-255.

36. The effects of benzodiazepines on cognition. Stewart, S. s.l. : Journal of clinical psychiatry, 2005, Vol. 66, pp. 9-13.

37. Justice, US Department of. Drug Enforcement Administration, benzodiazepines. US Department of Justice. [Online] [Cited: March 18, 2009.] http://www.usdoj.gov/dea/concern/benzodiazepines.html.

38. Bono, J. Criminalistics- Introduction to Controlled Substances. [ed.] S. Karch. Drug Abuse Handbook. s.1. : CRC Press, 1998, pp. 5-7. heroin.

39. Detection of Acetylcodeine in Urine as an Indicator of Illicit Heroin Use: Method Validation and Results of a Pilot Study. Staub, C., Marset, M. and al, et. 2, 2001, Clinical Chemistry, Vol. 47, pp. 301-307.

40. DEA Drug Scheduling. [Online] [Cited: July 26, 2009.]

http://www.justice.gov/dea/pubs/scheduling.html.

41. U.S. Department of Justice. [Online] [Cited: January 3, 2009.] morphine. http://www.usdoj.gov/dea/concern/morphine.html.

42. Baselt, R., [ed.]. Diposition of toxic chemicals and drugs in man. Foster City, CA : Biomedical Publications, 2004. pp. 678-681. methadone.

43. Oates, J. The Science of Drug Therapy. [ed.] L. Brunton. Goodman and Gilman's The pharmacological basis of therapeutics. 11th. New York : McGraw-Hill Medical Publishing Division, 2006, pp. 117-137. 
44. Postmortem forensic toxicology of trazodone. Goeringer, K., Raymon, L., et al. 4, 2000, J. Forensic Sci, Vol. 45, pp. 850-856.

45. Reference Concentrations of Antidepressants. A Compilation of Postmortem and Therapeutic Levels. Reis, M., Ahlner, J. and Druid, H. 2007, J. Anal. Toxicol, Vol. 31, pp. 255-263.

46. Postmortem redistribution of fentanyl in blood. Olson, K., Luckenbill, K., et al. 2010, AM J Clin Pathol, Vol. 133, pp. 447-453.

47. Postmortem assay of digoxin by radioimmunoassay. Holt, W. and Benstead, J. 1975, J. Clin. Pathol., Vol. 28, pp. 483-486.

48. Determination of therapuetic and toxic serum digoxin concentrations by radio immunoassay. Smith, T., Butler, V. and Haber, E. 1969, New Engl J Med, Vol. 281, pp. 1212-1216.

49. Interpretation of postmortem serum levels of cardiac glycosides after suspected overdosage. Moffat, A. 1974, Acta Pharmacologica et Toxicologica, Vol. 35, pp. 386394.

50. Digoxin concentrations in postmortem specimens after overdose and therapeutic use. DiMaio, V., Garriott, J. and Putnam, R. 2, 1975, J. Forensic Sci., Vol. 20, pp. 340-347.

51. Case experience with digoxin analysis of postmortem blood. Phillips, A. 1974, J FORENSIC SCI SOC, Vol. 14, pp. 137-140.

52. Correlation of antemortem and postmortem digoxin levels. Vorpahl T. and Coe, J. s.1. : J. Forensic Sci., 1978, Vol. 23, pp. 329-334.

53. Postmortem changes in serum levels of the tricyclic antidepressants. Bandt, C. Los Angeles, Ca : s.n., 1980. presented at the American Academy of Forensic Sciences Meeting.

54. Postmortem changes in drug concentrations in the blood. Kofoed, J. and Mayer, J. Niagra Falls, Canada : s.n., 1986. presented at the Canadian Society of Forensic Sciences Meeting.

55. Postmortem increase in drug levels- a major challenge for forensic toxicologists. Jones, G. Montreal, Quebec : s.n., 1985. presented at the joint meeting of the Society of Forensic Toxicologists and the Canadian Society of Forensic Sciences.

56. Documented hazards in the interpretation of postmortem blood concentration of tricyclic ntidepressants. Prouty, R. and Anderson, W. Anaheim, Ca : s.n., 1984. presented at the Americam Academy of Forensic Sciences. 
57. Distribution of ethanol in postmortem specimens. Briglia, E., Hauser, C. et al. Quantico, Va : s.n., 1986. presented at the International Symposium on Driving Under the Influence of Alcohol and/or Drugs.

58. Blood and tissue concentrations of tricyclic antidepressant drugs in postmortem cases. Literature survey and a study of forty deaths. Hebb, J., Caplan, Y. et al. 1982, J. Anal. Toxicol, Vol. 6, pp. 209-216.

59. Therapeutic and toxic drug concentrations in postmortem blood: A six year study in the State of Maryland. Caplan, Y., Ottinger, W. and Crooks, R. s.l. : J. Anal. Toxicol, September/October 1983, Vol. 7, pp. 225-230.

60. Concentration of basic drugs in human myocardium. Bailey, D. and Shaw, R. 2, 1982, J TOXICOL-CLIN TOXIC, Vol. 19, pp. 197-202.

61. Site dependence of drug concentrations in postmortem blood-A case study. Jones, G. and Pounder, D. s.l. : J. Anal. Toxicol, September/October 1987, Vol. 11, pp. 186-190.

62. Comparative study of postmortem barbiturates, methadone and morphine in vitreous humor, blood and tissue. Ziminski, K., Wemyss, C, et al. 3, 1984, J. Forensic Sci, Vol. 29, pp. 903-909.

63. The liver:blood ratio in cases of barbiturate poisoning. Curry, A. and Sunshine, I. 1960, Toxicol Appl Pharmacol, Vol. 2, pp. 602-606.

64. Physicians' Desk Reference. 60th. Montvale : Thompson PDR , 2006. p. 2822.

65. Stability of analytes in biosamples - an important issue in clinical and forensic toxicology. Peters, F. and bioanalytical chemistry : s.n., 2007, Analytical, Vol. 388, pp. 1505-1519.

66. Stability of drugs stored in postmortem femoral blood and vitreous humor. Holmgren, P. 4, 2004, Journal of Forensic Sciences, Vol. 49, pp. 820-825.

67. Preanalytical aspects in postmortem toxicolgy. Skopp, G. 142, s.1. : Forensic Science International, 2004, pp. 75-100.

68. Stability of morphine, morphine-3-glucuronide and morphine-6-glucuronide in fresh blood and plasma and postmortem blood samples. Skopp, G. January/February 2001, Journal of Analytical Toxicology, Vol. 25.

69. Miller, J. and Miller, J. Statistics and Chemometrics for Analytical Chemistry. 4th. s.1. : Prentice Hall, 2001. 
70. Fayetteville State University. [Online] [Cited: January 1, 2010.]

http://faculty.uncfsu.edu/dwallace/lesson\%2016.pdf.

71. Baselt, R., [ed.]. Disposition of toxic drugs and chemicals in man. 7. Foster City, CA : Biomedical Publications, 2004. pp. 262-264. codeine.

72. Baselt, R., [ed.]. Dispositon of toxic drugs and chemicals in man. 7. Foster City, CA : Biomedical publications, 2004. pp. 759-762. morphine.

73. Hutchins, K. Pathologist at MDME Dept. Miami, September 2009.

74. Health, US National Library of Medicaine and National Institutes of. Medline Plus: Medical Dictionary. [Online] [Cited: August 15, 2009.] http://www.nlm.nih.gov/medlineplus/mplusdictionary.html.

75. Health, US National Library of Medicine and the National Institute of. Medline Plus. [Online] [Cited: September 13, 2009.] diphenhydramine. http://www.nlm.nih.gov/medlineplus/druginfo/meds/a682539.html.

76. Baselt, R. Disposition of toxic drugs and chemicals in man. Foster City : Biomedical Publications, 2004. diphenhydramine.

77. The Vaults of Erowid. [Online] September 2, 2004. [Cited: September 13, 2009.] diphenhydramine. http://www.erowid.org.

78. Baselt, R., [ed.]. Disposition of toxic drugs and chemicals in man. 7. Foster City, CA : Biomedical Publications, 2004. pp. 35-36. Alprazolam.

79. - Disposition of toxic chemicals and drugs in man. 7th. Foster City : Biomedical Publications, 2004. pp. 312-315. diazepam.

80. EMS House of DeFrance. [Online] [Cited: December 5, 2009.] http://www.defrance.org/.

81. Baselt, R., [ed.]. Disposition of Toxic Drugs and Chemicals in Man. 7th. Foster City : Biomedical Publications, 2004. pp. 747-749. midazolam.

82. Disposition of toxic chemicals and drus in man. [ed.] R. Baselt. 7th. Foster City : Biomedical Publication, 2004, pp. 246-247. clonazepam.

83. Clonazepam pharmazokinetics, brain uptake and receptor interactions. Greenblatt, D, Miller, L and Shader, R. Supplement, s.1. : Journal of Clinical Psychiatry, October 1987, Vol. 48, pp. 4-11. 
84. U.S. Department of Justice. [Online] [Cited: January 3, 2009.] hydrocodone. http://www.usdoj.gov/dea/concern/hydrocodone.html.

85. Blood concentrations of clonazepam and 7-aminoclonazepam in forensic cases in Denmark for the period 2002 - 2007. Steentoft, A. and Linnet, K. 184, s.1. : Forensic Science International, 2009, pp. 74-79. 
SAMANTHA SHIRELLE TOLLIVER

\author{
May 6, $1979 \quad$ Born, Hagerstown, MD \\ B.S., Chemistry \\ West Virginia State University \\ Institute, WV \\ 2005 \\ M.S., Chemistry \\ Florida International University \\ Miami, FL \\ 2005 \\ Toxicologist \\ Miami-Dade County Medical Examiner Department \\ Miami, FL \\ 2006 - $2010 \quad$ Doctoral Candidate in Chemistry \\ Florida International University \\ Miami, FL
}

\title{
PUBLICATIONS AND PRESENTATIONS
}

Recipient of the Society of Forensic Toxicology's Educational Research Award 2010.

Tolliver, S., Hearn, W.L., and Furton, K.G. (2010). Identifying Variances between Postmortem and Antemortem Morphine and Codeine Concentrations. Presented at the Society of Forensic Toxicology's $40^{\text {th }}$ Annual Meeting.

Tolliver, S., Hearn, W.L., and Furton, K.G. (2010). Evaluating the Relationship between Postmortem and Antemortem Morphine and Codeine Concentrations in Whole Blood. Accepted for the Special Edition of Journal of Analytical Toxicology 2010.

Tolliver, S., Hearn, W.L., and Furton, K.G. (2008). Evaluating the Significance of Variation of Drug concentrations in Antemortem and Postmortem Blood Samples. Presented at the American Academy of Forensic Sciences $60^{\text {th }}$ Annual Scientific Meeting. 